\title{
Timing-Based Localization using Multipath Information
}

\section{Andreas Bergström}




\section{Timing-Based Localization using Multipath Information}

\section{Andreas Bergström}

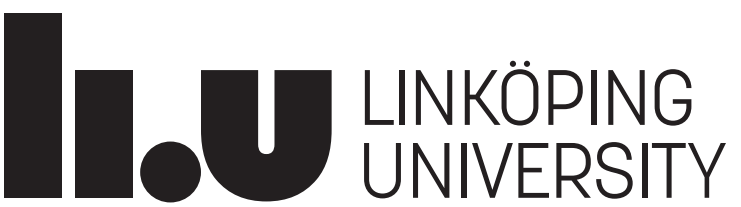


This is a Swedish Licentiate's Thesis.

Swedish postgraduate education leads to a Doctor's degree and/or a Licentiate's degree.

A Doctor's Degree comprises 240 ECTS credits (4 years of full-time studies).

A Licentiate's degree comprises 120 ECTS credits,

of which at least 60 ECTS credits constitute a Licentiate's thesis.

Linköping studies in science and technology. Licentiate Thesis

No. 1867

Timing-Based Localization using Multipath Information

Andreas Bergström

andreas.bergstromeliu.se

www. control.isy.liu.se

Department of Electrical Engineering

Linköping University

SE-581 83 Linköping

Sweden

ISBN 978-91-7929-917-0ＩSSN 0280-7971

Copyright (C) 2019 Andreas Bergström

Printed by LiU-Tryck, Linköping, Sweden 2019 
Till Kerstin och Oskar! 



\section{Abstract}

The measurements of radio signals are commonly used for localization purposes where the goal is to determine the spatial position of one or multiple objects. In realistic scenarios, any transmitted radio signal will be affected by the environment through reflections, diffraction at edges and corners etc. This causes a phenomenon known as multipath propagation, by which multiple instances of the transmitted signal having traversed different paths are heard by the receiver. These are known as Multi-Path Components (MPCs). The direct path (DP) between transmitter and receiver may also be occluded, causing what is referred to as non-Line-of-Sight (non-LOS) conditions. As a consequence of these effects, the estimated position of the object(s) may often be erroneous.

This thesis focuses on how to achieve better localization accuracy by accounting for the above-mentioned multipath propagation and non-LOS effects. It is proposed how to mitigate these in the context of positioning based on estimation of the DP between transmitter and receiver. It is also proposed how to constructively utilize the additional information about the environment which they implicitly provide. This is all done in a framework wherein a given signal model and a map of the surroundings are used to build a mathematical model of the radio environment, from which the resulting MPCs are estimated.

First, methods to mitigate the adverse effects of multipath propagation and non-LOS conditions for positioning based on estimation of the DP between transmitter and receiver are presented. This is initially done by using robust statistical measurement error models based on aggregated error statistics, where significant improvements are obtained without the need to provide detailed received signal information. The gains are seen to be even larger with up-to-date real-time information based on the estimated MPCs.

Second, the association of the estimated MPCs with the signal paths predicted by the environmental model is addressed. This leads to a combinatorial problem which is approached with tools from multi-target tracking theory. A rich radio environment in terms of many MPCs gives better localization accuracy but causes the problem size to grow large - something which can be remedied by excluding less probable paths. Simulations indicate that in such environments, the single best association hypothesis may be a reasonable approximation which avoids the calculation of a vast number of possible hypotheses. Accounting for erroneous measurements is crucial but may have drawbacks if no such are occurring.

Finally, theoretical localization performance bounds when utilizing all or a subset of the available MPCs are derived. A rich radio environment allows for good positioning accuracy using only a few transmitters/receivers, assuming that these are used in the localization process. In contrast, in a less rich environment where basically only the DP/LOS components are measurable, more transmitters/receivers and/or the combination of downlink and uplink measurements are required to achieve the same accuracy. The receiver's capability of distinguishing between multiple MPCs arriving approximately at the same time also affects the localization accuracy. 



\section{Populärvetenskaplig sammanfattning}

Var hittar sköterskan närmaste dialysapparat? Vilka platser i kontorslandskapet är upptagna? Hur hittar roboten sin last i lagerlokalen? Var la jag min klocka? Radiobaserad positionering är en möjliggörare i dessa och många andra krävande exempel på positionering i inomhusmiljöer där satellitbaserad positionering inte är möjlig. Genom mätningar av utsända radiosignaler kan en mottagare bestämma egenskaper hos signalen såsom t.ex. fördröjning, signalstyrka och/eller riktning för att på så sätt uppskatta avståndet till sändaren. Med flera sådana mätningar är det (åtminstone konceptuellt) relativt enkelt att lista ut var det eftersökta föremålet finns. Radiosignaler påverkas dock av omgivningen på flera olika sätt, de reflekteras i fönster, väggar och andra föremål, dämpas genom väggar osv. Det leder bland annat till att mottagaren tar emot flera olika kopior av samma skickade signal, fast med olika fördröjningar och signalstyrkor - ett fenomen som kallas flervägsutbredning. Det kan i sin tur göra att de uppmätta avstånden blir felaktiga, och således även slutsatsen om var föremålet finns.

Den här avhandlingen handlar om just detta: Hur använder man den extra information en mottagare faktiskt får när den tar emot en signal som utsatts för sådan flervägsutbredning för att få bättre positioneringsnoggrannhet? Först presenteras en generell modell av en sådan radiokanal samt de nödvändiga verktyg som behövs för att ta reda på fördröjning och signalstyrka hos den mottagna signalen över alla de olika vägar den färdats. Därefter behandlas tre olika ämnen:

Först studeras valet av mätfelsmodeller när man på försöker hitta och använda den kortaste vägen (fågelvägen) mellan sändare och mottagare, då bättre kunskap om dessa fel kan förbättra lokaliseringsprestandan. Slutsatsen är att man med relativt enkla felmodeller baserade på aggregerad statistik kan få tydliga förbättringar och med tillgång till mer aktuella mätningar så är vinsterna ännu större. Så också med filtrering av mätdata över tid snarare än en rent momentan skattning. Den uppnådda prestandan är dock fortfarande inte så bra som önskat, varför det föreslås att använda mer kanalinformation såsom diskuterats ovan.

Därefter visas hur man kan para ihop mätningarna av de flera mottagna signalkopiorna och de möjliga signalvägar som finns. Detta formuleras som ett klassiskt tilldelningsproblem, där det visar sig att den bästa enskilda hypotesen i allmänhet kan vara en tillräckligt bra approximation som undviker beräkning av många möjliga hypoteser. En rik radiomiljö med många mottagna signalkopior ger bättre prestanda. Samtidigt ökar problemets storlek och komplexitet, vilket kan minskas genom att utesluta de mest osannolika signalvägarna. Det visar sig avgörande att ta hänsyn till mätfel. Att kombinera mätningar från flera sändare och/eller mottagare är fördelaktigt, speciellt i en mindre rik radiomiljö.

Slutligen utvärderar vi den teoretiska prestanda man kan förvänta sig när man använder just denna flervägsutbredningsrelaterade information. Återigen visas att hur rik radiomiljön är starkt påverkar vilken noggrannhet man kan få. Detta påverkas även av hur väl mottagaren kan särskilja olika signalkopior som anländer ungefär samtidigt. Även här ser vi att en kombination av mätningar mellan flera sändare och/eller mottagare samt även mellan upplänks- och nedlänkssändingar ytterligare kan förbättra lokaliseringsprestandan. 



\section{Acknowledgments}

The path to success is rarely straight, someone once said. Beginning to pursue a $\mathrm{PhD}$ at the age of 38, was perhaps not the most convenient career move to make. A colleague of mine frankly told me: "You are a fool! Why put this burden on yourself? Why not be content with the great job you already have?" To some extent he was right: The amount of blood, sweat and tears I have put into my PhD studies resembles nothing I have ever done before. Accompanied by a rather complicated personal life during this period, well... I did not make it particularly easy for myself, one might say.

Still I do not regret that I took this opportunity when presented. Not for a single second! I have learned a tremendous amount during this journey. Not only on technical topics, but maybe more importantly about myself and the people around me. How easy it is to take things for granted is one such lesson. The difference between research and engineering is another one, where I many times have a tendency of being more of the latter. I have indeed made more than a few suboptimal decisions along the way. Still, I cannot see how I could have done differently, and am happy and proud of having reached all the way here. I make no claims of this thesis to be a great one. But it is mine..!

However, none of it would not have been possible, if it had not been for a whole lot of people. Those who where there already from the start as well as those I have had the privilege to meet along the way. I want to thank all of you for making this possible!

I would like to start by expressing my deepest gratitude towards my main supervisor Fredrik Gustafsson as well as my co-supervisors Gustaf Hendeby and Fredrik Gunnarsson. Thank you all for the encouragement, inspiration, helpfulness and commitment you have shown me over the last few years. And patience... This thesis could never have been possible if it not had been for the three of you!

I would also like to thank Svante Gunnarsson and Martin Enqvist, as well as all my other awesome colleagues here at Automatic Control for creating a professional, inspiring and enjoyable work environment and for making this group a pleasure to be part of - both during and outside office hours. In particular, I would like to thank Gustav Lindmark, Per Boström-Rost, Kristoffer Bergman, Anton Kullberg and Magnus Malmström for reading and providing valuable input to the manuscript of this thesis.

My employer Ericsson and then especially Gunnar Bark, my previous manager at Ericsson Research, deserves a lot of credit for giving me the opportunity to pursue this path as an industrial PhD student, despite some initial scepticism. My current manager Nicklas Johansson also deserves much credit for his continued support along the way. Both of you are indeed the best, most supportive and down-to-earth managers one could ever have hoped for. I want to believe that I have learned something from you. Thank you! And to all my colleagues at LinLab - too many to name here but you all know who you are - thank you for making all these years with you a joy! I can hardy imagine better colleagues and a better and more inspiring place to work at! 
This work was supported by the Wallenberg AI, Autonomous Systems and Software Program (WASP) funded by the Knut and Alice Wallenberg Foundation. Their funding is gratefully acknowledged and appreciated. All the interesting and inspiring people I have met and listened to within the WASP context are countless. Special thanks to all the batch one students for good times during conferences and trips as well as to the WASP Graduate School Management with Fredrik Heintz, Patric Jensfelt and others for making this a great journey!

I owe many and massive thanks to my family and to my friends. You all know who you are. Even though I have a tendency of taking for granted the love and support you give me, that does not mean that I am not grateful and glad to have you all in my life. I will, and believe that I can, show this appreciation better in the future..!

And Gunilla, we have had a journey much longer than that of this thesis. We have had our ups and downs and, even though we are no longer 'we', I still consider you my best and closest friend and hope to be able continue doing so. Thank you for everything!

Last, but absolutely not least: Kerstin and Oskar, my beloved children. Thank you for putting up with a father who sometimes is so stressed out and preoccupied with himself and his projects that he forgets to fully appreciate how wonderful you are. Thank you for making me the best father I can ever hope to be. You are the lights of my life and what makes everything matter! I cannot, and do not ever want to, imagine what life would be without you! I love you more than words can say! Puss!

Linköping, December 2019 Andreas Bergström 


\section{Contents}

Notation

xiii

1 Introduction 1

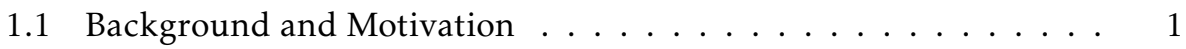

1.2 Research Questions . . . . . . . . . . . . . . . . . . 4 4

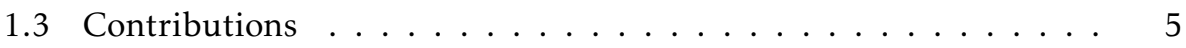

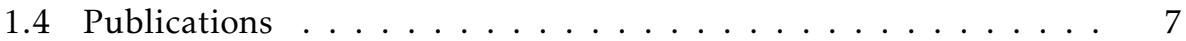

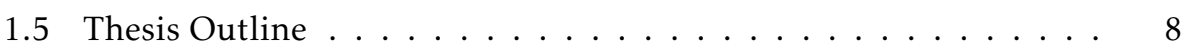

2 Modelling 9

2.1 Scenario Descriptions . . . . . . . . . . . . . . . . . . 9

2.2 Radio Channel Modelling . . . . . . . . . . . . . . . 11

2.2.1 Multiple-Input-Multiple-Output (MIMO) . . . . . . . . . 11

2.2.2 Single-Input-Single-Output (SISO) $\ldots \ldots \ldots \ldots \ldots$

2.3 Geometric Modelling . . . . . . . . . . . . . . . . . 16

2.4 Signal Model . . . . . . . . . . . . . . . . . . . . 21

2.5 Power Delay Profile (PDP) $\ldots \ldots \ldots \ldots \ldots \ldots \ldots \ldots$

3 Estimation and Measurements 27

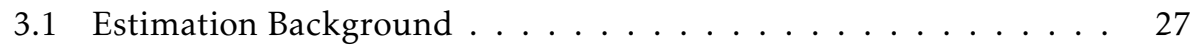

3.1 .1 Parameter Estimation . . . . . . . . . . . . . . . . 27

3.1 .2 State Estimation . . . . . . . . . . . . . . . 28

3.2 Estimation of Channel Parameters / MPCs . . . . . . . . . . 30

3.3 Full Pseudo-Range Measurements . . . . . . . . . . . . . . 32

3.3 .1 Uni-Directional . . . . . . . . . . . . . . . . . 32

3.3 .2 Bi-Directional . . . . . . . . . . . . . . . . 34

3.4 Estimation of Agent Location $\ldots \ldots \ldots \ldots \ldots$

4 TOA Estimation and Measurement Error Modelling 37

$4.1 \mathrm{DP} /$ LOS Estimation and Measurements . . . . . . . . . . . 38

4.2 Error Models . . . . . . . . . . . . . . . . . . . . 40

4.2.1 Static Error Models . . . . . . . . . . . . . . . . 40

4.2 .2 Dynamic Error Models . . . . . . . . . . . . . . . 43 
4.3 Numerical Results . . . . . . . . . . . . . . . . . . . . 46

4.3.1 Snapshot Estimation Results . . . . . . . . . . . . . . . . 47

4.3.2 Filtering Results . . . . . . . . . . . . . . 50

4.4 Summary ........................... 52

4.A Additional Figures . . . . . . . . . . . . . 53

5 The Measurements Association Problem $\quad 67$

5.1 Problem Formulation . . . . . . . . . . . . . . . 67

5.2 Measurements Association . . . . . . . . . . . . . 68

5.2.1 Mapping from Measurements to MPCs/VAs . . . . . . . 68

5.2 .2 Multiple Hypotheses . . . . . . . . . . . . . . . . . 68

5.3 Hypothesis Reduction . . . . . . . . . . . . . . . 70

5.3.1 Finding the Best Hypothesis . . . . . . . . . . . 70

5.3.2 Finding the B-Best Hypotheses . . . . . . . . . . . 71

5.3.3 Gating of Measurements . . . . . . . . . . . 72

5.4 Numerical Results . . . . . . . . . . . . . . . . . . . . 72

5.4.1 Measurement Assumptions . . . . . . . . . . 72

5.4.2 Estimation Models . . . . . . . . . . . . . . . 74

5.4.3 Approximation by using the Single Best Hypothesis . . . . 75

5.4.4 Results under Realistic Model Conditions . . . . . . . . . 78

5.4.5 Results under Simplified Model Conditions . . . . . . . . . 81

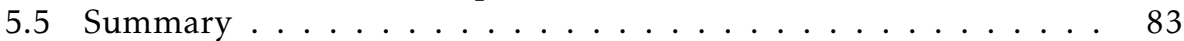

5.A Additional Figures . . . . . . . . . . . . . . 84

6 Performance Bounds 95

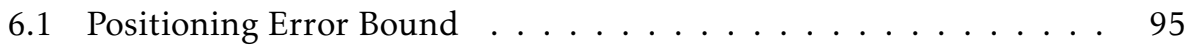

6.1 .1 Impact of Path Overlap . . . . . . . . . . . . . . 97

6.1.2 Limited Observations . . . . . . . . . . . . . . . 98

6.1.3 Separate DL and UL Measurements . . . . . . . . . . . . . . . 99

6.1.4 Combined DL and UL Measurements . . . . . . . . . . . . 100

6.2 Numerical Results . . . . . . . . . . . . . . . . . . . . 100

6.2.1 Impact of Path Overlap . . . . . . . . . . . . . . . . 101

6.2 .2 Limited Observations . . . . . . . . . . . . . . . 104

6.2.3 Combined DL and UL Measurements . . . . . . . . . . 107

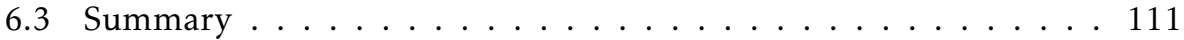

7 Concluding Remarks $\quad 113$

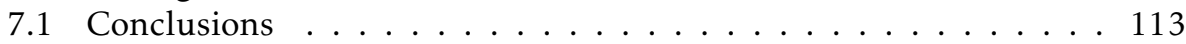

7.2 Future Work ......................... 114

$\begin{array}{ll}\text { Bibliography } & 115\end{array}$ 
Notation

\section{Abbreviations}

\begin{tabular}{cl}
\hline Abbreviation & Meaning \\
\hline 3GPP & 3rd Generation Partnership Project \\
5G & 5th Generation Mobile Networks \\
AWGN & Additive White Gaussian Noise \\
AOA & Angle of Arrival \\
AOD & Angle of Departure \\
BLUE & Best Linear Unbiased Estimator \\
BS & Base Station \\
CDF & Cumulative Density Function \\
CIR & Channel Impulse Response \\
CRLB & Cramér-Rao Lower Bound \\
CV & Constant Velocity \\
DL & Downlink \\
DM & Diffuse Multipath \\
DP & Direct Path \\
EFIM & Equivalent Fisher Information Matrix \\
FA & False Alarm \\
FIM & Fisher Information Matrix \\
FTM & Fine Timing Measurements \\
GMM & Gaussian Mixture Model \\
GNN & Global Nearest Neighbour \\
GNSS & Global Navigation Satellite System \\
GPS & Global Positioning System \\
IEEE & Institute of Electrical and Electronics Engineers \\
IOT & Internet of Things \\
ITU & International Telecommunication Union \\
JV & Jonker-Volgenant \\
LD & Late Detection \\
LOS & Line-of-Sight \\
LS & Least Squares \\
&
\end{tabular}




\begin{tabular}{|c|c|}
\hline Abbreviation & Meaning \\
\hline LTE & Long Term Evolution \\
\hline LTV & Linear Time Varying \\
\hline MAP & Maximum A Posteriori \\
\hline MC & Monte Carlo \\
\hline MD & Missed Detection \\
\hline MIMO & Multiple-Input Multiple-Output \\
\hline ML & Maximum Likelihood \\
\hline MMSE & Minimum Mean Square Error \\
\hline MPC & Multi-Path Component \\
\hline MVU & Minimum Variance Unbiased \\
\hline NLOS & Non Line-of-Sight \\
\hline $\mathrm{NN}$ & Nearest Neighbour \\
\hline NR & New Radio Access \\
\hline OTDOA & Observed Time Difference of Arrival \\
\hline PEB & Positioning Error Bound \\
\hline PDF & Probability Density Function \\
\hline PDP & Power Delay Profile \\
\hline $\mathrm{PF}$ & Particle Filter \\
\hline PSD & Power Spectral Density \\
\hline RMSE & Root Mean Square Error \\
\hline RTT & Round-Trip Time \\
\hline $\mathrm{RX}$ & Receiver \\
\hline SIR & Sequential Importance Resampling \\
\hline SINR & Signal-to-Interference-and-Noise Ratio \\
\hline SISO & Single-Input Single-Output \\
\hline SLAM & Simultaneous Localization and Mapping \\
\hline SNR & Signal-to-Noise Ratio \\
\hline TDOA & Time Difference of Arrival \\
\hline TOA & Time of Arrival \\
\hline TOF & Time-of-Flight \\
\hline $\mathrm{TX}$ & Transmitter \\
\hline UE & User Equipment \\
\hline UL & Uplink \\
\hline US & Uncorrelated Scattering \\
\hline UWB & Ultra Wideband \\
\hline VA & Virtual Anchor \\
\hline WASP & $\begin{array}{l}\text { Wallenberg AI, Autonomous Systems and Software } \\
\text { Program }\end{array}$ \\
\hline WIFI & Wireless Fidelity \\
\hline WLS & Weighted Least Squares \\
\hline WSS & Wide-Sense Stationary \\
\hline WSSUS & Wide-Sense Stationary Uncorrelated Scattering \\
\hline
\end{tabular}




\section{1}

\section{Introduction}

This chapter is intended to provide the reader with an introduction to the key topic of this thesis, i.e., timing-based localization using multipath information. It posts the research questions to be answered and further highlights the contributions and publications provided by this thesis. Finally, it attempts to make the thesis more accessible by outlining its structure and the interplay of the different parts of which it consists.

\subsection{Background and Motivation}

Localization, also referred to as positioning, addresses the problem on how to estimate the spatial position of one or multiple objects. The objects could be pretty much anything, such as e.g. mobile phones, people, cars, air planes, space shuttles, robots, missiles, wildlife animals, etc. The localization could be amended with a temporal aspect for which the considered object(s) are tracked over time. Various techniques can be used for these purposes like vision-oriented systems using cameras, various kinds of dead-reckoning, inertial measurements as well as measurements of both acoustic and electromagnetic waves.

Radio-based localization deals with how to use measurements of electromagnetic waves in the radio spectrum, i.e., radio signals, for the purpose of localizing and tracking of objects. A receiver here tries to determine certain properties of a received signal such as, for instance the amplitude and/or the delay from transmission to reception. These properties can thereafter be used to find the location of the considered object(s), which typically is the transmitter and/or receiver. Much research has been focused on positioning using UWB ranging (see e.g. [12]), partly because of its attractive signal properties, but also because the availability of equipment for experiments. 
Positioning support in terrestrial cellular networks has historically been driven by regulatory requirements for emergency calls but is nowadays considered a key component in a wide range of applications, from crude to very accurate positioning. The increasing interest in Internet of Things (IOT) and the development of 5G (see e.g. [36, 49]) also brings requirements for ubiquitous connectivity and positioning. Use-case discussed in this context includes everything from to the roughly knowing the location of goods or fleet vehicles all the way to real-time tracking of high-speed ground/aerial vehicles or the pose estimation of remotely operated surgical equipment. For an overview of different methods for positioning in wireless networks, see $[17,37,38]$ and the more recent $[49]$.

Many positioning methods in wireless networks are based on Time-of-Arrival (TOA) estimation of a received signal with known characteristics, where the delay (which is proportional to the distance) between transmitter and receiver is estimated. TOA measurements and estimation is analyzed in different contexts in $[6,9,23,41]$. Multiple TOA estimates can then be used to localize the object(s) as discussed in $[14,16,17]$. Any transmitted radio signal will be affected by the surrounding environment in numerous ways. Consider e.g. an indoor office scenario where the signal is reflected in windows, walls and other objects, attenuated through walls and experiences diffraction at corners and edges. This creates what is referred to as a multipath fading radio channel, wherein the receiver receives multiple copies, called Multi-Path Components (MPCs), of the same transmitted radio signal. The different MPCs have various degrees of delays and/or attenuation as discussed in $[13,46]$. Furthermore, the MPC corresponding to the Direct Path (DP) or Line-of-Sight (LOS) path, i.e., the path with the shortest Euclidean distance between transmitter and receiver, may be occluded. This causes what is known as non-LOS conditions (see e.g. [21]). How to model these propagation effects as well as how to estimate the MPCs is discussed in this thesis.

A simple illustration of TOA-based positioning and the impact of multipath propagation for a case with two transmitters and one receiver in an idealized case without noise or other types of errors is given in Figure 1.1 below. Here

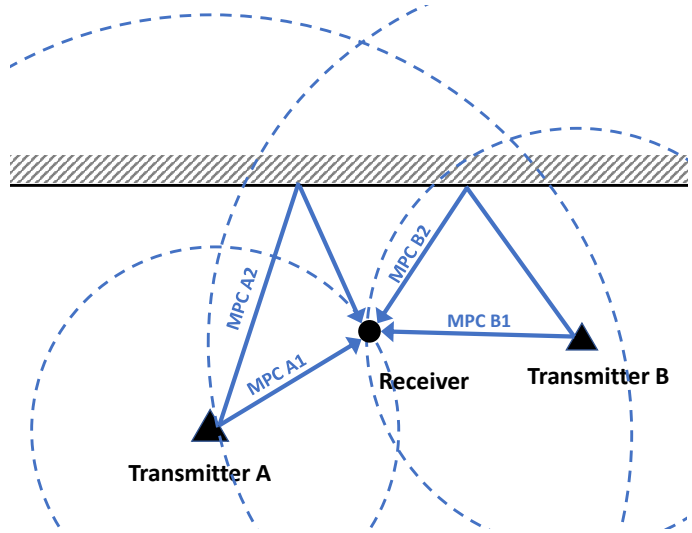

(a) Overview

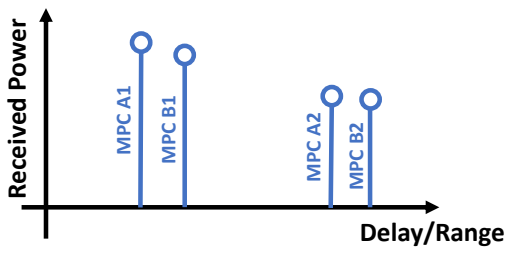

(b) Estimated MPCS

Figure 1.1: Illustration of the MPCs and TOA estimation in a simple case. 
the transmitted signals are reflected on a wall, causing the receiver to receive not only the DP/LOS components 'MPC A1' and 'MPC B1', but also the reflected MPCs 'MPC A2' and 'MPC B2'. Having determined the delay of the respective MPCs (and hence also the range) as in Figure 1.1b, it can assume the distance to be as per the dashed circles in Figure 1.1a. Any intersection of these circles will then be a reasonable position estimate of the receiver. However, these circles do not intersect one another in one place but gives an ambiguity in where this position may be. This is partly due to the fact that the receiver cannot know which of the MPCs that are to be associated with the DP/LOS path. In this simple case, one option would of course be to use only the MPCs with the shortest delays, i.e., 'MPC A1' and 'MPC B1'. That would however still create ambiguities since the corresponding circles still intersects in two places. Adding more transmitters may be possible but is a non-generic (and sometimes expensive) solution which is not expected to work properly in the presence of noise, with other placement of the transmitters and/or receiver etc. and may hence still give ambiguities.

Because of the above mentioned multipath and/or non-LOS conditions, the resulting TOA estimates will thus be erroneous in some aspect. The receiver may e.g. associate the wrong MPC with the DP/LOS path and hence an erroneous delay/distance between the transmitter and receiver. These estimation errors will therefore impair the subsequent localization accuracy. One way to deal with this is to attempt to accurately model these measurement errors as discussed e.g. in [43] and [4] - a topic which is covered by this thesis.

Rather than treating these environmental effects on the signal as errors as discussed above, another possible approach is instead to utilize more of the information in the multipath radio channel. For example, a signal received after having been reflected in an object implicitly says something about the location of that object in relation to the transmitter and/or receiver. Assume that the room layout is known a priori. Then, upon receiving the different MPCs of the transmitted signal, one can potentially conclude which surfaces the signal was reflected on and use this additional information to improve the position estimate. This method has been proposed and investigated in e.g. $[7,29]$ assuming the geometry of the environment to be known a priori and also using Simultaneous Localization and Mapping (SLAM) techniques ([2, 8]) in e.g. [25]. The theoretical localization performance bounds achievable when utilizing this multipath-related information in the radio channel is considered in e.g. $[7,29,44]$ and is also further revisited in this thesis.

Returning to the example of Figure 1.1, but now assuming that we can indeed utilize this multipath information with knowledge about the points at which 'MPC A2' and 'MPC B2' were reflected, we get the situation depicted in Figure 1.2. Here, the dashed circles all intersect at one single point and hence there is no ambiguity in where the receiver is. The reflection points of 'MPC A2' and 'MPC B2' in all essence become 'virtual transmitters' that can be used for TOA estimation. This approach is used in e.g. [29], albeit there the mirrored image of the respective transmitter on the opposite side of the reflective surface are used and referred to as virtual anchors (VAs). 


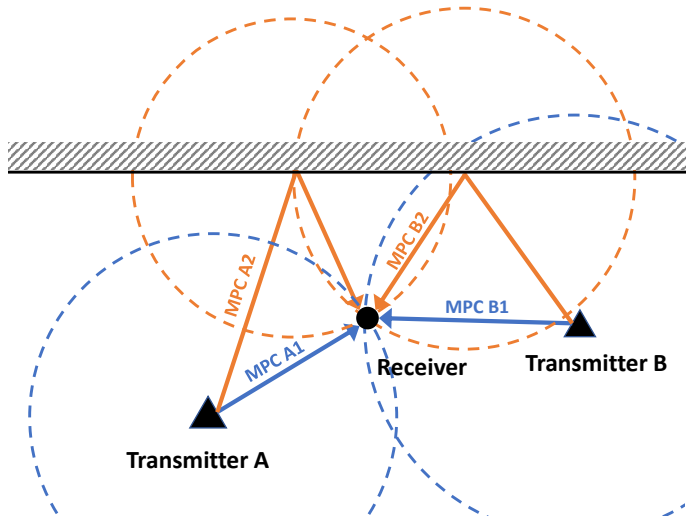

(a) Overview

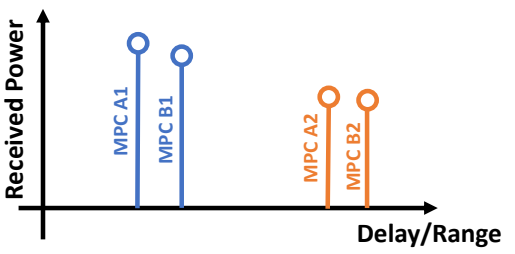

(b) Estimated MPCS

Figure 1.2: Illustration of the MPCs and TOA estimation when being able to utilize the multipath information.

With this approach for utilizing the multipath information, it is however crucial to be able to associate the different received MPCs with the correct transmission paths (and hence to the various objects of environment) also in the presence of measurement noise, non-LOS conditions and other types of errors. It is impossible for the receiver to directly distinguish between the multiple received MPCs, but it still needs to somehow be able to perform this association - a topic which is also addressed in this thesis.

\subsection{Research Questions}

The aim of this thesis is to investigate how to utilize the multiple MPCs of a multipath fading radio channel for the purpose of better localization accuracy. In particular, we seek to provide answers to the following research questions:

- How can we predict and estimate the different MPCs of a multipath fading radio channel? This includes not only the DP/LOS component but also those having traversed other paths.

- When using localization based on estimated distances between the physical transmitters and receivers, i.e., the DP/LOS component, how can we then mitigate the localization errors caused by e.g. multipath propagation and non-LOS conditions?

- How can we correctly associate the estimated MPCs with the actual traversed signal paths, and hence to an environmental model, so that we can use them all for localization purposes?

- What is the theoretical localization performance bounds achievable when utilizing more or less of the additional information provided by the estimated MPCs? 


\subsection{Contributions}

The main contributions of this thesis are:

1. A set of measurement error models for DP/LOS estimation which mitigates the adverse effects of multipath propagation and non-LOS conditions.

2. Associating the estimated MPCs with the actual traversed signal paths using tools from multi-target tracking theory. This makes it possible to constructively utilize the additional information about the environment implicitly provided by these MPCs.

3. Providing theoretical localization performance bounds when utilizing all or a subset of the available MPCs in different scenarios. This is done under varying assumptions on receiver modelling as well as combination of measurements from different sources.

In addition, the author of this thesis has put much effort into the overall framework presented in Chapters 2 and 3. It contains an UWB signal model as well a model of a generic multipath fading radio channel, which in turn explicitly depends on an environmental description such as e.g. a map given by the two provided indoor scenarios. It also contains the tools required to estimate the resulting MPCs of this radio channel. This framework is to a large extent based on the significant work of others as per the therein listed references (e.g. $[7,29,30])$ and is therefore not considered a contribution as such in this thesis.

The first contribution considers the choice of measurement error models when estimating the DP/LOS path between the transmitter and receiver. It is shown that much of the adverse effects of multipath propagation and non-LOS conditions can be mitigated with models based on aggregated statistics, without the need to provide detailed received signal information but with a rather generic model. The gains are even larger with up-to-date real-time information based on the estimated MPCs. This contribution is the topic of Chapter 4, which is an extension of the published paper [4]. As compared to [4], this chapter uses the more refined models and framework provided by this thesis, estimates the location rather than just the distances, develops error models based on collected statistics as well as those being dynamic/time-varying rather than those which are only static and also applies filtering in addition to mere snapshot estimation.

The second contribution deals with the association of the estimated MPCs with the signal paths predicted by the environmental model. This leads to a combinatorial problem which is approached with tools from multi-target tracking theory. It is formulated as a classical assignment problem, also accounting for erroneous measurements of various sorts, which is solved using a Global Nearest Neighbour (GNN) method. It is shown that a rich radio environment in terms of many MPCs gives not only better localization accuracy, but also causes the association problem size to grow large - something which can to some extent be remedied by the exclusion of less probable paths. Simulations also indicate that in rich radio environments, the single best association hypothesis can be a reasonable approximation which avoids the calculation of a vast number of possible hypotheses. 
Accounting for erroneous measurements is shown crucial if such are occurring but may have drawbacks otherwise. Combining measurements from multiple sources is shown beneficial, since this increases the amount of information available in the estimation process. This contribution is covered by Chapter 5 .

The third and final contribution is the derivation of the theoretical localization performance bounds when utilizing all or a subset of the available MPCs. It is confirmed that a rich radio environment with many measurable MPCs allows for good accuracy using only few transmitters/receivers, assuming that these are indeed used in the localization process. In contrast, in a less rich environment where basically only the MPCs corresponding to the DP/LOS path(s) are measurable, more transmitters/receivers and/or the combination of downlink (DL) and uplink (UL) measurements are required in order to achieve the same localization accuracy. The receiver's capability of distinguishing between multiple MPCs arriving approximately at the same time to the receiver is also shown to affect the localization accuracy. This contribution is assessed in Chapter 6.

For easier accessibility of this thesis, an overview of how the different contributions, chapters, sections and topics of this thesis are interconnected is shown in Figure 1.3. Here also the logical flow of data, together with references to the appropriate chapters and sections, is indicated.

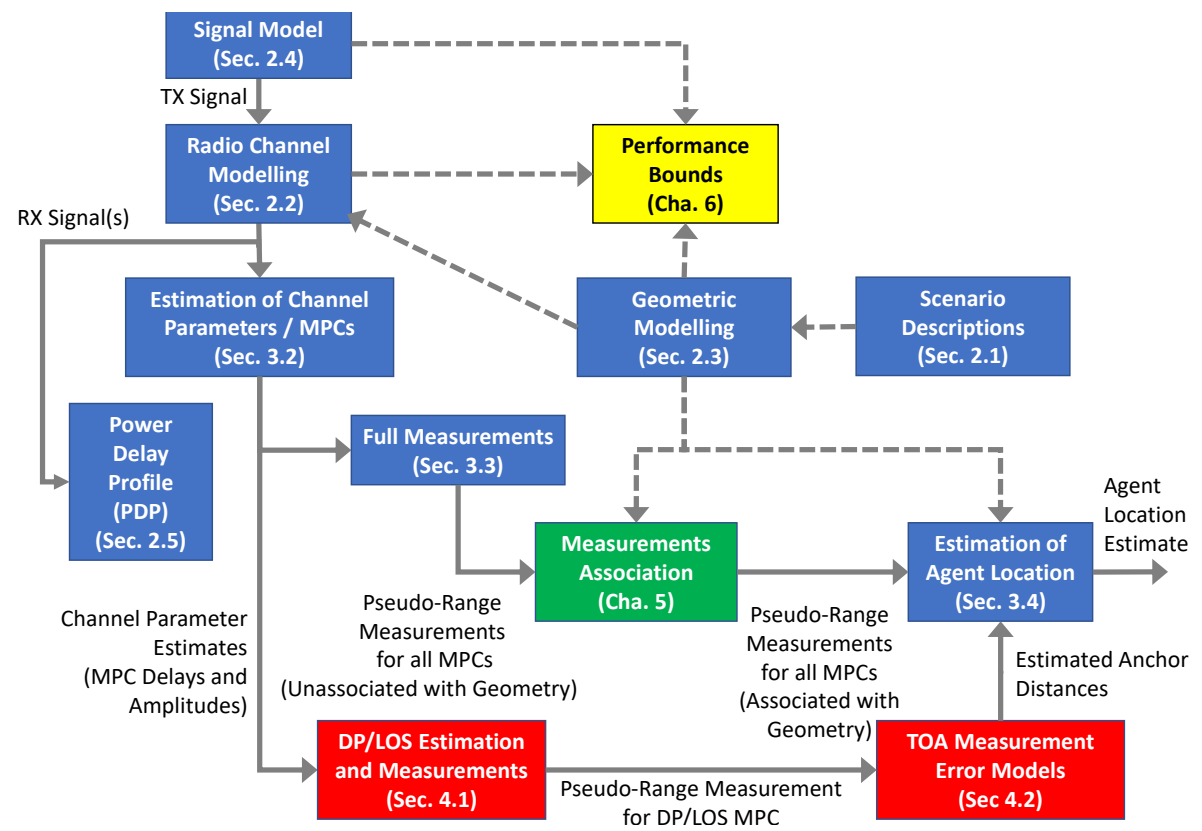

Figure 1.3: An overview of the logical flow of thesis together with references to the appropriate chapters and sections. Topics related to the general framework of this thesis are shown in blue whereas those related to the first contribution are shown in red, to the second contribution in green and to the third contribution in yellow. 


\subsection{Publications}

The first publication [4] considers measurement error models for DP/LOS ranging estimation:

A. Bergström, G. Hendeby, F. Gunnarsson, and F. Gustafsson. TOA estimation improvements in multipath environments by measurement error models. In 2017 IEEE 28th Annual International Symposium on Personal, Indoor, and Mobile Radio Communications (PIMRC), pages 1-7, Montreal, QC, CA, Oct. 2017.

In this publication we conclude that more detailed information about the error distribution implies significant ranging estimation improvement. However, very detailed information about individual paths does not improve the results compared to a simpler model of the error distribution in the studied scenarios. As mentioned in the previous section, the first contribution of this thesis covered by Chapter 4 is an extension of this publication.

In addition, the author of this thesis has published another paper [47], which is not directly related to the contents of this thesis:

D. Verenzuela, A. Bergström, and E. Björnson. Optimal Power Control for Superimposed Pilots in Uplink Massive MIMO Systems. In 2018 IEEE International Conference Record of the 52nd Asilomar Conference on Signals Systems and Computers, pages 499-503, Pacific Grove, CA, USA, Oct. 2018.

The author's contribution to this paper was in discussions with the first author of the paper, as well as in the writing of the manuscript. 


\subsection{Thesis Outline}

This rest of this thesis is organized as follows:

Chapter 2: Modelling gives an overview of the considered scenarios and introduces the used signal and radio channel models. It shows how the latter is derived from an environmental/geometrical model, including calculations of the MPCs and the Power Delay Profile (PDP).

Chapter 3 : Estimation and Measurements introduces a few standard methods for estimation such as the maximum likelihood (ML) estimator and the particle filter (PF). These are used to estimate the essential channel parameters corresponding to the MPCs subsequently needed as input to the also herein described full delay/range measurements. Finally, the estimation of the agent location based on these measurements is discussed.

Chapter 4 : TOA Estimation and Measurement Error Modelling discusses TOA estimation and measurements relating to the DP/LOS path, i.e., the shortest path between transmitter and receiver. Several measurement error models are derived for this purpose and it is evaluated what impact these have on the resulting localization accuracy.

Chapter 5 : The Measurements Association Problem deals with how to properly perform the association of the estimated MPCs with the respective transmission paths of the radio channel. This combinatorial problem is formulated as a classical assignment problem using a multi-target tracking approach, which can be solved using standard methods. This chapter also discusses how to reduce the size and complexity of this problem.

Chapter 6 : Performance Bounds investigates the theoretical localization performance bounds achievable when utilizing the multipath-related information, i.e., the complete or partial set of MPCs in the radio channel.

Chapter 7 : Concluding Remarks contains the conclusions as well as thoughts on future research possibilities within the area.

The reader is also referred to the earlier Figure 1.3 in Section 1.3 for an overview of how these different chapters and topics are interconnected. 


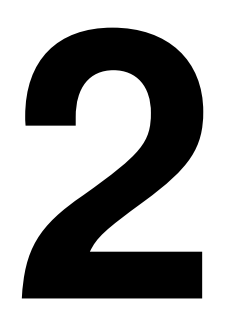

\section{Modelling}

This chapter establishes the scenarios and models used throughout this thesis and is organized as follows: First, Section 2.1 describes the two considered indoor scenarios. Thereafter, Section 2.2 presents a model of a generic multipath fading radio channel, which in turn explicitly depends on an environmental description (such as e.g. the two provided indoor scenarios) as described in Section 2.3. The considered UWB signal model is presented in Section 2.4, whereafter Section 2.5 discusses estimation of the Power Delay Profile (PDP).

\subsection{Scenario Descriptions}

In this thesis, we consider the two indoor scenarios depicted in Figure 2.1 below. Scenario A is a crooked room of $10 \times 8$ meters containing a large pillar of $2 \times 2$ me-

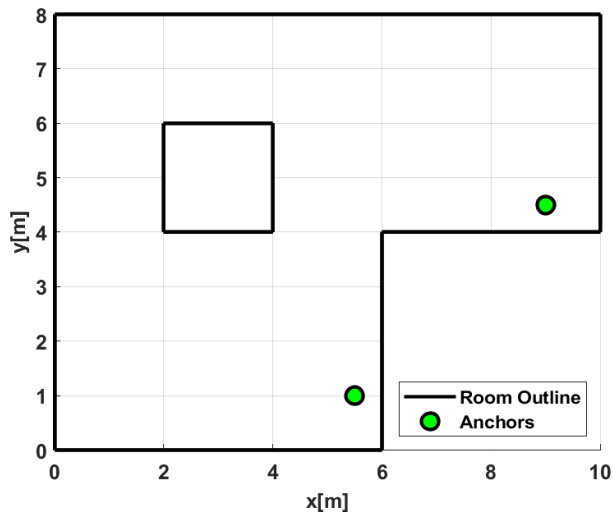

(a) Scenario $A$

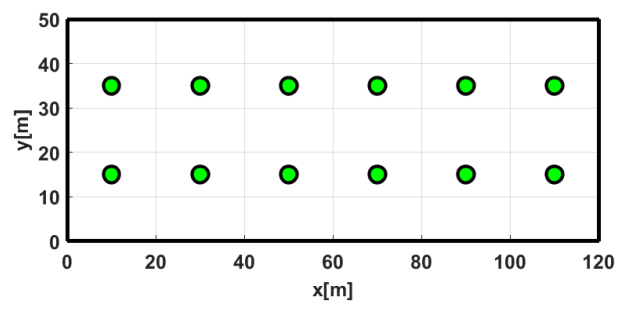

(b) Scenario $B$

Figure 2.1: The two different scenarios considered in this thesis. 


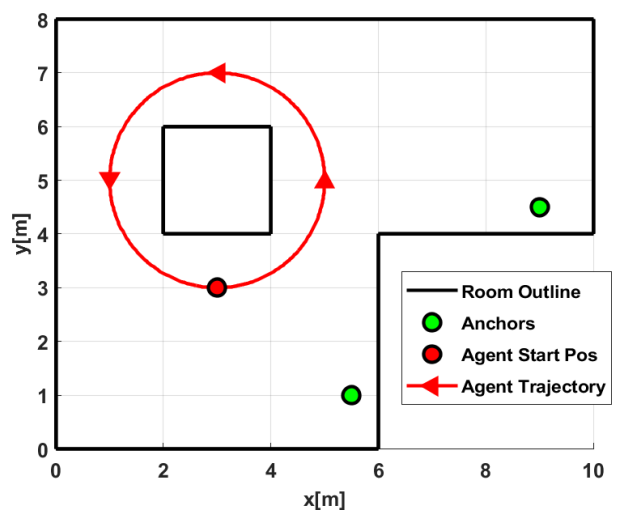

Figure 2.2: The circular agent trajectory for Scenario A.

ters and with 2 anchors at positions $\mathbf{p}_{1}=[5.5,9.1]^{T}$ and $\mathbf{p}_{2}=[9,4.5]^{T}$. Scenario $B$ is influenced by work done in 3GPP [1] with a large rectangular room of $120 \times 50$ meters without pillars or obstacles. The 12 anchors are in this scenario placed in a rectangular grid 20 meters apart at $\mathbf{p}_{1}=[10,15]^{T}, \mathbf{p}_{2}=[10,35]^{T}, \mathbf{p}_{3}=[30,15]^{T}$ etc. These two scenarios are used in the geometric modelling discussed in Section 2.3, where we assume (if not stated otherwise) that all anchors as well as any agents are equipped with one isotropic antenna each (see Section 2.2.2).

Additionally, we also consider a circular trajectory in Scenario A where the agent starts at the position $[3,3]^{T}$ and moves counter-clockwise in a full circle with radius 2 meters and centred at $[3,5]^{T}$ as in Figure 2.2 above. Whenever this trajectory is used, we refer to the 'Position Index' or 'Position Along Trajectory' as the angle in degrees counted counter-clockwise relative the position where the agent started. Hence, 'Position Index' 0 is at $[3,3]^{T}$ whereas 'Position Index' 90 is at $[5,5]^{T}$ etc.

The full set of scenario parameters are given in Table 2.1 below, where we also indicate in which section of this thesis the respective parameter is discussed.

Table 2.1: Scenario Parameters

\begin{tabular}{|c|c|c|c|c|}
\hline Parameter & Value & Description & Comment & Section \\
\hline$f_{c}$ & $7 \mathrm{GHz}$ & Carrier Frequency & Same in DL \& UL & 2.4 \\
$s(t)$ & As per $(2.27)$ & Signal & UWB Raised Cosine & 2.4 \\
$T$ & $1 \mathrm{~ns}$ & Pulse Duration & - & 2.4 \\
$\beta$ & 0.6 & Roll-off & - & 2.4 \\
$T_{S}$ & $10 \mathrm{ps}$ & Sampling Time & 100×Oversampling & 2.5 \\
$N_{0}$ & $10^{-8}$ & AWGN Noise & High SNR case & 2.2 \\
$N_{0}$ & $10^{-6}$ & AWGN Noise & Low SNR case & 2.2 \\
$\gamma_{\text {rise }}$ & $5 \mathrm{~ns}$ & DM parameter 1 & - & 2.2 \\
$\gamma_{1}$ & $20 \mathrm{~ns}$ & DM parameter 2 & - & 2.2 \\
$\chi$ & 0.98 & DM parameter 3 & - & 2.2 \\
$\max (Q)$ & 3 & Max MPC Order & Max nr Reflections & 2.3 \\
$\Delta_{A}$ & $5 \mathrm{~cm}$ & Grid Res. Scenario A & Same in x,y & - \\
$\Delta_{B}$ & $50 \mathrm{~cm}$ & Grid Res. Scenario B & Same in x,y & - \\
\hline
\end{tabular}




\subsection{Radio Channel Modelling}

This section provides a basic but general model of a Multiple-Input MultipleOutput (MIMO) radio channel, which is thereafter simplified to that of SingleInput-Single-Output (SISO) radio channel.

\subsubsection{Multiple-Input-Multiple-Output (MIMO)}

Consider a system with one transmitter (TX) having $M_{T}$ transmit antennas and one receiver (RX) with $M_{R}$ receive antennas as depicted in Figure 2.3.

The complex-valued baseband signal sent by the transmitter at time $t$ is

$$
\mathbf{s}(t)=\left[s_{1}(t), s_{2}(t), \ldots, s_{M_{T}}(t)\right]^{T},
$$

where $s_{a}(t)$ is the signal transmitted by antenna $a \in\left\{1, \ldots, M_{T}\right\}$ located at position $\mathbf{p}_{a}$. Similarly, the receiver at time $t$ observes the signal

$$
\mathbf{r}(t)=\left[r_{1}(t), r_{2}(t), \ldots, r_{M_{R}}(t)\right]^{T},
$$

where $r_{b}(t)$ is the signal received by antenna $b \in\left\{1, \ldots, M_{R}\right\}$ located at $\mathbf{p}_{b}$.

The radio channel between the transmitter and the receiver can be modelled as a Linear Time-Varying (LTV) system ([46], [32] and [13]),

$$
\mathbf{r}(t)=\int_{\tau=0}^{\infty} \mathbf{H}(t, \tau) \mathbf{s}(t-\tau) d \tau+\mathbf{w}(t),
$$

where $\tau$ is the time delay of the radio channel and $\mathbf{w}(t)$ is additive noise. A common assumption is that this noise is zero mean circular symmetric complex Gaussian (AWGN) with a positive definite covariance matrix $\Sigma \in \mathbb{R}^{M_{R} \times M_{R}}$. The probability density function (PDF) of $\mathbf{w}$ becomes in this case

$$
\mathcal{C N}(\mathbf{w} ; \mathbf{0}, \Sigma)=\frac{1}{\pi^{M_{R}} \operatorname{det} \Sigma} \exp \left\{-\mathbf{w}^{H} \Sigma^{-1} \mathbf{w}\right\}
$$

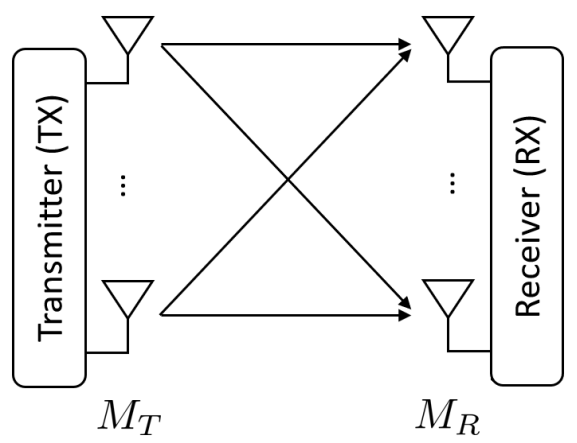

Figure 2.3: A generic MIMO scenario with one transmitter having $M_{T}$ transmit antennas and one receiver having $M_{R}$ receive antennas. 
Another common assumption is that $\Sigma=N_{0} \mathbf{I}_{M_{R}}$ where $\mathbf{I}_{M_{R}}$ is the $M_{R} \times M_{R}$ identity matrix and $N_{0}$ is the power spectral density (PSD) of the noise, which thus implies that the noise is assumed mutually uncorrelated between the receive antennas.

The time-variant channel impulse response $\mathbf{H}(t, \tau) \in \mathbb{C}^{M_{R} \times M_{T}}$ in (2.3) describes the radio channel between each pair of antenna elements as

$$
\mathbf{H}(t, \tau)=\left[\begin{array}{ccc}
h_{1,1}(t, \tau) & \cdots & h_{1, M_{T}}(t, \tau) \\
\vdots & \ddots & \vdots \\
h_{M_{R}, 1}(t, \tau) & \cdots & h_{M_{R}, M_{T}}(t, \tau)
\end{array}\right]
$$

where $h_{b, a}(t, \tau)$ is the channel impulse response between TX antenna $a$ and RX antenna $b$. The channel impulse response $\mathbf{H}(t, \tau)$ can be calculated (see e.g. [28], [39]) as

$$
\mathbf{H}(t, \tau)=\iint\left[\mathbf{C}_{R X}\left(\rho_{R X}\right)\right]^{T} \mathbf{G}\left(t, \tau, \rho_{T X}, \rho_{R X}\right)\left[\mathbf{C}_{T X}\left(\rho_{T X}\right)\right] d \rho_{T X} d \rho_{R X} .
$$

Above, $\rho_{T X}$ and $\rho_{R X}$ are the angle of departure at the TX side and angle of arrival at the RX side, respectively. Dependent on these are $C_{R X} \in \mathbb{C}^{2 \times M_{R}}$ and $\mathbf{C}_{T X} \in \mathbb{C}^{2 \times M_{T}}$ which are the receive and transmit antenna response matrices, describing the complex electric field patterns of the respective antennas. Finally, $\mathbf{G}\left(t, \tau, \rho_{T X}, \rho_{R X}\right) \in \mathbb{C}^{2 \times 2}$ is the, assumed reciprocal, bi-directional impulse response kernel/function of the radio channel.

The transmitted signal $\mathbf{s}(t)$ will typically be subject to multipath propagation due to scattering, diffraction and/or reflections in the environment. This causes the signal to have traversed not only the shortest direct path (DP) (also referred to as the line-of-sight (LOS) path) but also typically several other paths as illustrated in Figure 2.4. As a result, multiple differently attenuated and phase-shifted variants of the transmitted signal, i.e., the MPCs, are received. For more details see e.g. [13].

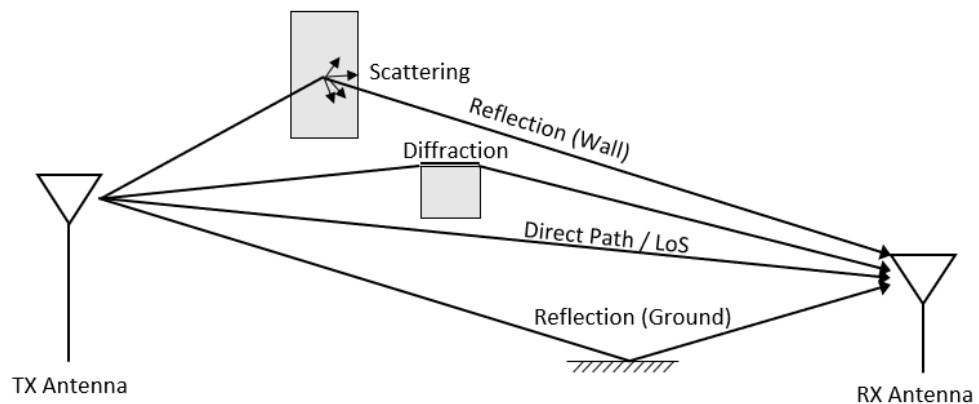

Figure 2.4: Illustration of direct, reflected, diffracted and scattered multipath components (MPCs). 
Assuming $K_{b, a}$ such MPCs between transmit antenna $a$ and receive antenna $b$, the channel impulse response $h_{b, a}(t, \tau)$ of (2.5) can be decomposed as

$$
h_{b, a}(t, \tau)=\sum_{k=1}^{K_{b, a}} h_{b, a}^{(k)}(t, \tau)
$$

where, in light of (2.6), the channel impulse response function between transmit antenna $a$ and receive antenna $b$ for MPC $k$ becomes

$$
h_{b, a}^{(k)}(t, \tau)=\iint\left[\mathbf{C}_{R X, b}\left(\rho_{R X}\right)\right]^{T} \mathbf{G}_{b, a}^{(k)}\left(t, \tau, \rho_{T X}, \rho_{R X}\right)\left[\mathbf{C}_{T X, a}\left(\rho_{T X}\right)\right] d \rho_{T X} d \rho_{R X}
$$

ans where $\mathbf{C}_{R X, b}$ and $\mathbf{C}_{T X, a}$ denotes the $b$ :th and $a$ :th columns of the respective matrices. The quantity $\mathbf{G}_{b, a}^{(k)}\left(t, \tau, \rho_{T X}, \rho_{R X}\right)$ in (2.8) is the bi-directional impulse response kernel/function of the radio channel for MPC $k$ between TX antenna $a$ and RX antenna $b$. In this thesis, we do not dig into the details of these quantities. Rather, we make a series of assumptions:

Assumption 1: All MPCs are specular reflections [28, 39], which gives

$$
\mathbf{G}_{b, a}^{(k)}\left(t, \tau, \rho_{T X}, \rho_{R X}\right)=\mathbf{A}^{(k)} e^{-i 2 \pi v^{(k)} t} \delta\left(\tau-\tau_{b, a}^{(k)}\right) \delta\left(\rho_{T X}-\rho_{T X}^{(k)}\right) \delta\left(\rho_{R X}-\rho_{R X}^{(k)}\right)
$$

where $\mathbf{A}^{(k)}$ is a complex $2 \times 2$ polarization matrix, $v^{(k)}$ is the Doppler frequency, $\delta(\cdot)$ is the Dirac delta function and $\tau_{b, a}^{(k)}$ is the time delay of the MPC. Furthermore, $\rho_{T X}^{(k)}$ and $\rho_{R X}^{(k)}$ are the angle-of-departure (AOD) and angle-of-arrival (AOA), respectively. Inserted into (2.8) this gives

$$
h_{b, a}^{(k)}(t, \tau)=\left[\mathbf{C}_{R X, b}\left(\rho_{R X}^{(k)}\right)\right]^{T} \mathbf{A}^{(k)}\left[\mathbf{C}_{T X, a}\left(\rho_{T X}^{(k)}\right)\right] e^{-i 2 \pi v^{(k)} t} \delta\left(\tau-\tau_{b, a}^{(k)}\right) .
$$

Assumption 2: All scattering is performed by stationary objects, and hence the Doppler shift is zero for all MPCs, i.e.,

$$
v^{(k)}=0, \quad \Rightarrow \quad e^{-i 2 \pi v^{(k)} t}=1 \quad \forall k .
$$

Assumption 3: All antennas are isotropic, so that the polarization and directionality of the respective antenna becomes irrelevant, i.e.,

$$
\left[\mathbf{C}_{R X, b}\left(\rho_{R X}^{(k)}\right)\right]^{T} \mathbf{A}^{(k)}\left[\mathbf{C}_{T X, a}\left(\rho_{T X}^{(k)}\right)\right]=\alpha_{b, a}^{(k)} \quad \forall k
$$

where $\alpha_{b, a}^{(k)} \in \mathbb{C}$ is a complex-valued scalar referred to as the complex MPC amplitude. If the transmitter sends a known signal, i.e., a pilot sequence, its contribution to the received signal can be included in this scalar.

Under Assumptions 1-3, and further allowing the complex amplitude $\alpha_{b, a}^{(k)}$ as well as the time delay $\tau_{b, a}^{(k)}$ to slowly vary over time $t$, the channel impulse response of (2.7) becomes the well-known tapped-delay line model (see e.g. [32]),

$$
h_{b, a}(t, \tau)=\sum_{k=1}^{K_{b, a}} \alpha_{b, a}^{(k)}(t) \delta\left(\tau-\tau_{b, a}^{(k)}(t)\right) .
$$


As discussed in e.g. [24], [22], [11] and [27], one may model the channel impulse response $h_{b, a}(t, \tau)$ as consisting of not only the $K_{b, a}$ deterministic MPCs as in (2.13), which typically caters for the direct path and reflections of the environmental/geometrical model (Section 2.3). In addition, a diffuse multipath (DM) component can be added, in order to cater for scattering, diffraction and other effects not explained by the environmental/geometrical model. By introducing the random variable $v_{b, a}(t)$, this turns $(2.13)$ into

$$
h_{b, a}(t, \tau)=\sum_{k=1}^{K_{b, a}} \alpha_{b, a}^{(k)}(t) \delta\left(\tau-\tau_{b, a}^{(k)}(t)\right)+v_{b, a}(t)
$$

whereby the full channel impulse response matrix of (2.5) with all pairs of TX and RX antenna combinations becomes

$$
\begin{aligned}
& \mathbf{H}(t, \tau)=\left[\begin{array}{ccc}
\sum_{k=1}^{K_{1,1}} \alpha_{1,1}^{(k)}(t) \delta\left(\tau-\tau_{1,1}^{(k)}(t)\right) & \ldots & \sum_{k=1}^{K_{1, M_{T}}} \alpha_{1, M_{T}}^{(k)}(t) \delta\left(\tau-\tau_{1, M_{T}}^{(k)}(t)\right) \\
\vdots & \ddots & \vdots \\
K_{M_{R}, 1} \alpha_{M_{R}, 1}^{(k)}(t) \delta\left(\tau-\tau_{M_{R}, 1}^{(k)}(t)\right) & \ldots & \sum_{k=1}^{K_{M_{R}, M_{T}}} \alpha_{M_{R}, M_{T}}^{(k)}(t) \delta\left(\tau-\tau_{M_{R}, M_{T}}^{(k)}(t)\right)
\end{array}\right]+\ldots \\
& \ldots+\mathbf{H}^{D E T}(t, \tau) \\
& \underbrace{\left[\begin{array}{ccc}
v_{1,1}(t) & \ldots & v_{1, M_{T}}(t) \\
\vdots & \ddots & \vdots \\
v_{M_{R}, 1}(t) & \cdots & v_{M_{R}, M_{T}}(t)
\end{array}\right]}_{\mathbf{H}^{D M}(t)}=\mathbf{H}^{D E T}(t, \tau)+\mathbf{H}^{D M}(t) .
\end{aligned}
$$

Here, $\mathbf{H}(t, \tau)$ has thus been split into one deterministic part $\mathbf{H}^{D E T}(t, \tau)$ and one diffuse-multipath part $\mathbf{H}^{D M}(t)$, which both are discussed further below.

The first term in (2.14), and the elements of $\mathbf{H}^{D E T}(t, \tau)$ in (2.15), describe a sum over the $K_{b, a}$ deterministic MPCs with complex amplitudes $\left\{\alpha_{b, a}^{(k)}(t)\right\}$ and delays $\left\{\tau_{b, a}^{(k)}(t)\right\}$. More details on the computation of these deterministic MPCs from an environmental/geometric model are given in Section 2.3.

The second term in (2.14), and the elements of the matrix $\mathbf{H}^{D M}(t)$ in (2.15), models the diffuse multipath components. It is defined as a zero-mean Gaussian random process with an auto-correlation function $\mathbb{E}_{v_{b, a}}\left\{v_{b, a}(\tau)\left[v_{b, a}(u)\right]^{*}\right\}=$ $S_{v_{b, a}}(\tau) \delta(\tau-u)$. Here, $S_{v_{b, a}}(\tau)$ is the power-delay profile (PDP) of the DM with respect to the antenna-pair $(b, a)$ (see Section 2.5). This means that the scattering components are modelled as mutually uncorrelated along the delay axis $\tau$ (i.e., between different MPCs), but assumed quasi-stationary in the spatial domain (i.e., over a close geographical neighbourhood). In this thesis, we model it as in $[7,19]$ where the DM impairs the signal only on the delay interval $\tau \in\left[\tau_{b, a}^{\min }(t), \infty\right]$, where

$$
\tau_{b, a}^{\min }(t)=\min _{\tau_{b, a}}\left\{\tau_{b, a}^{(k)}(t): k=1, \ldots, K_{b, a}\right\}
$$


is the delay of the first detected MPC with respect to the antenna-pair $(b, a)$ at time $t$, i.e.,

$$
S_{v_{b, a}}(\tau)= \begin{cases}S^{0}\left(\tau-\tau_{b, a}^{\min }(t)\right) & \tau \geq \tau_{b, a}^{\min }(t) \\ 0 & \text { otherwise }\end{cases}
$$

for which

$$
S^{0}(\tau)=\Omega_{1} \frac{\gamma_{1}+\gamma_{\text {rise }}}{\gamma_{1}\left(\gamma_{1}+\gamma_{\text {rise }}(1-\chi)\right)}\left(1-\chi e^{-\tau / \gamma_{\text {rise }}}\right) e^{-\tau / \gamma_{1}}
$$

The parameters $\gamma_{\text {rise }}, \gamma_{1}, \chi$ and $\Omega_{1}$ of $(2.17)$ we use in this thesis are given in Table 2.1 of Section 2.1.

The resulting received signal (2.3) given the models (2.14), (2.16) and (2.17) with respect to TX antenna $a$ and RX antenna $b$ now becomes

$$
r_{b, a}(t)=\sum_{k=1}^{K_{b, a}} \alpha_{b, a}^{(k)}(t) s_{a}\left(t-\tau_{b, a}^{(k)}(t)\right)+v_{b, a}(t) \star s_{a}(t)+w_{b}(t)
$$

where $\star$ denotes convolution and $w_{b}(t) \sim \mathcal{N}\left(0, N_{0} / 2\right)$. Considering all TX antennas, the full received signal at RX antenna $b$ becomes

$$
r_{b}(t)=\sum_{a=1}^{M_{T}}\left(\sum_{k=1}^{K_{b, a}} \alpha_{b, a}^{(k)}(t) s_{a}\left(t-\tau_{b, a}^{(k)}(t)\right)+v_{b, a} \star s_{a}(t)+w_{b}(t)\right)
$$

and, consequently, the full received signal over all RX antennas is given by (2.2), with $r_{b}(t)$ as in (2.19) above.

\subsubsection{Single-Input-Single-Output (SISO)}

In the subsequent chapters of this thesis we assume only one transmit and one receive antenna, i.e., $M_{T}=M_{R}=1$. Hence the MIMO radio channel described in Section 2.2.1 becomes a Single-Input Single-Output (SISO) system and the antenna-pair indices $(b, a)$ can be discarded. The resulting channel impulse response (2.14) becomes

$$
h(t, \tau)=\sum_{k=1}^{K} \alpha^{(k)}(t) \delta\left(\tau-\tau^{(k)}(t)\right)+v(t)
$$

whereas the received signal (c.f. (2.18) and (2.19)) becomes

$$
r(t)=\sum_{k=1}^{K} \alpha^{(k)}(t) s\left(t-\tau^{(k)}(t)\right)+v \star s(t)+w(t) .
$$

For brevity in notation we will also from this point discard the explicit usage of the time index $t$ for the channel amplitudes and delays with respect to MPC $k$ and hence simply denote these as $\left\{\alpha^{(k)}\right\}$ and $\left\{\tau^{(k)}\right\}$, respectively. 


\subsection{Geometric Modelling}

Given knowledge of the environment (i.e., a map as per the scenarios described in Section 2.1), one can employ the concept of virtual anchors (VAs) described in e.g. $[7,29,30])$ to model the specular reflected deterministic MPCs. This models each MPC $k=1, \ldots, K_{b, a}$ arriving at RX antenna $b$ located at position $\mathbf{p}_{b}$ as originating from a set of $K_{b, a}$ VAs at positions $\left\{\mathbf{p}_{a}^{(k)}\right\}$, rather than from the TX antenna position $\mathbf{p}_{a}$ via a series of specular reflections.

Each VA is here associated with an order $Q^{(k)}$ corresponding to the number of reflections the related MPC $k$ has undergone. The zeroth order $\left(Q^{(k)}=0\right)$ VA corresponds simply to the DP/LOS component. The first order $\left(Q^{(k)}=1\right)$ VAs are acquired by mirroring the TX antenna positions $\left\{\mathbf{p}_{a}\right\}$ in the walls at which single reflections of the MPC occur. Second order $\left(Q^{(k)}=2\right)$ VAs are obtained by mirroring the first order VAs in a second reflected wall of the MPC etc.

We further define $\mathcal{K}_{b, a}^{\text {visible }}$ as the subset of all $K_{b, a}$ MPCs/VAs that are visible at the RX antenna location $\mathbf{p}_{b}$, i.e., those which have an unobstructed path between the VA at $\mathbf{p}_{a}^{(k)}$ and the RX antenna at $\mathbf{p}_{b}$,

$$
\mathcal{K}_{b, a}^{\text {visible }}=\left\{k: \mathbf{p}_{a}^{(k)}-\mathbf{p}_{b} \text { is unobstructed }, k=1, \ldots, K_{b, a}\right\} \subseteq\left\{1, \ldots, K_{b, a}\right\} .
$$

For all visible MPCs $k \in \mathcal{K}_{b, a}^{\text {visible }}$, the delays in (2.13) and (2.14) are given by

$$
\tau_{b, a}^{(k)}=\frac{1}{c}\left\|\mathbf{p}_{a}^{(k)}-\mathbf{p}_{b}\right\|_{2}=\frac{1}{c} d_{b, a}^{(k)} \quad \forall k \in \mathcal{K}_{b, a}^{\text {visible }}
$$

where $c$ is the speed of light and $d_{b, a}^{(k)}$ the Euclidean distance between the location $\mathbf{p}_{a}^{(k)}$ for VA $k$ of TX antenna $a$ and the location $\mathbf{p}_{b}$ of RX antenna $b$.

The transmitted pulse $s(t)$ is assumed to have unit energy, as discussed in Section 2.4. Hence, the received power of the MPCs are

$$
\left|\alpha_{b, a}^{(k)}\right|=10^{P_{b, a}^{(k)} / 20}
$$

for which the path loss $P_{L_{b, a}^{(k)}}$ is derived using a standard free-space propagation model (see e.g. [13]) with the addition of $3 \mathrm{~dB}$ attenuation per reflection,

$$
P_{L_{b, a}^{(k)}}=-20 \log _{10}\left(\frac{4 \pi d_{b, a}^{(k)}}{\lambda_{c}}\right)-3 Q^{(k)} \quad \forall k \in \mathcal{K}_{b, a}^{\text {visible }}
$$

where $\lambda_{c}=c / f_{c}$ is the carrier wavelength for a carrier frequency $f_{c}$. For any nonvisible MPC/VA $k \notin \mathcal{K}_{b, a}^{\text {visible }}$ we assume $\left|\alpha_{b, a}^{(k)}\right| \equiv 0$, i.e., full blocking of the signal whenever the path is obstructed.

The corresponding phase is given by the fraction of extra wavelengths over the distance $d_{b, a}^{(k)}(t)$ plus a phase shift of $\pi$ radians for each reflection,

$$
\angle \alpha_{b, a}^{(k)}=2 \pi\left(d_{b, a}^{(k)}-\left\lfloor\frac{d_{b, a}^{(k)}}{\lambda_{c}}\right\rfloor \lambda_{c}\right)+\pi Q^{(k)} \quad \forall k \in \mathcal{K}_{b, a}^{\text {visible }}
$$


Note that this model does not include the diffraction or scattering illustrated in Figure 2.4. These are instead included in the DM component $v_{b, a}(t)$ of $(2.14)$ through (2.19). Further, if considering transmissions in the opposite direction from antenna $b$ to antenna $a$ one can after some contemplation conclude that the MPCs and hence also the set of visible VAs by necessity will have to be the same. This is of course conditioned that the channel is reciprocal, which is the case if $\mathrm{TX} / \mathrm{RX}$ positions remain unchanged, that their respective antennas as well as the transmitter/receiver algorithms and impairments are identical and also the same frequency band is used. In this work we assume for simplicity that this is all true.

Example 2.1

Consider Scenario A described in Section 2.1, for which we assume two agents located at $\mathbf{x}_{1}=[3,3]^{T}$ and $\mathbf{x}_{2}=[1,7]^{T}$, respectively. The reflected signal paths, i.e., the MPCs are shown in Figure 2.5 whereas all the $K_{b, a}$ potential VAs are shown in Figure 2.6, considering reflections up to the order of three $\left(\max Q^{(k)}=3\right)$. The visible VAs of $\mathcal{K}_{b, a}^{\text {visible }}$ corresponding to the MPCs of Figure 2.5 are shown in Figure 2.7. The total number of visible MPCs/VAs at any given point of the room is shown in Figure 2.8, from which it can be seen that for each of the two anchors individually this number can be rather low in some regions. However, considering both anchors jointly, there are always at least around ten visible VAs. The resulting received powers vs. range for all MPCs, i.e., $\left\{\left|\alpha_{b, a}^{(k)}\right|^{2}\right\}$ and $\left\{d_{b, a}^{(k)}\right\}=\left\{c \tau_{b, a}^{(k)}\right\}$ are shown in Figure 2.9.
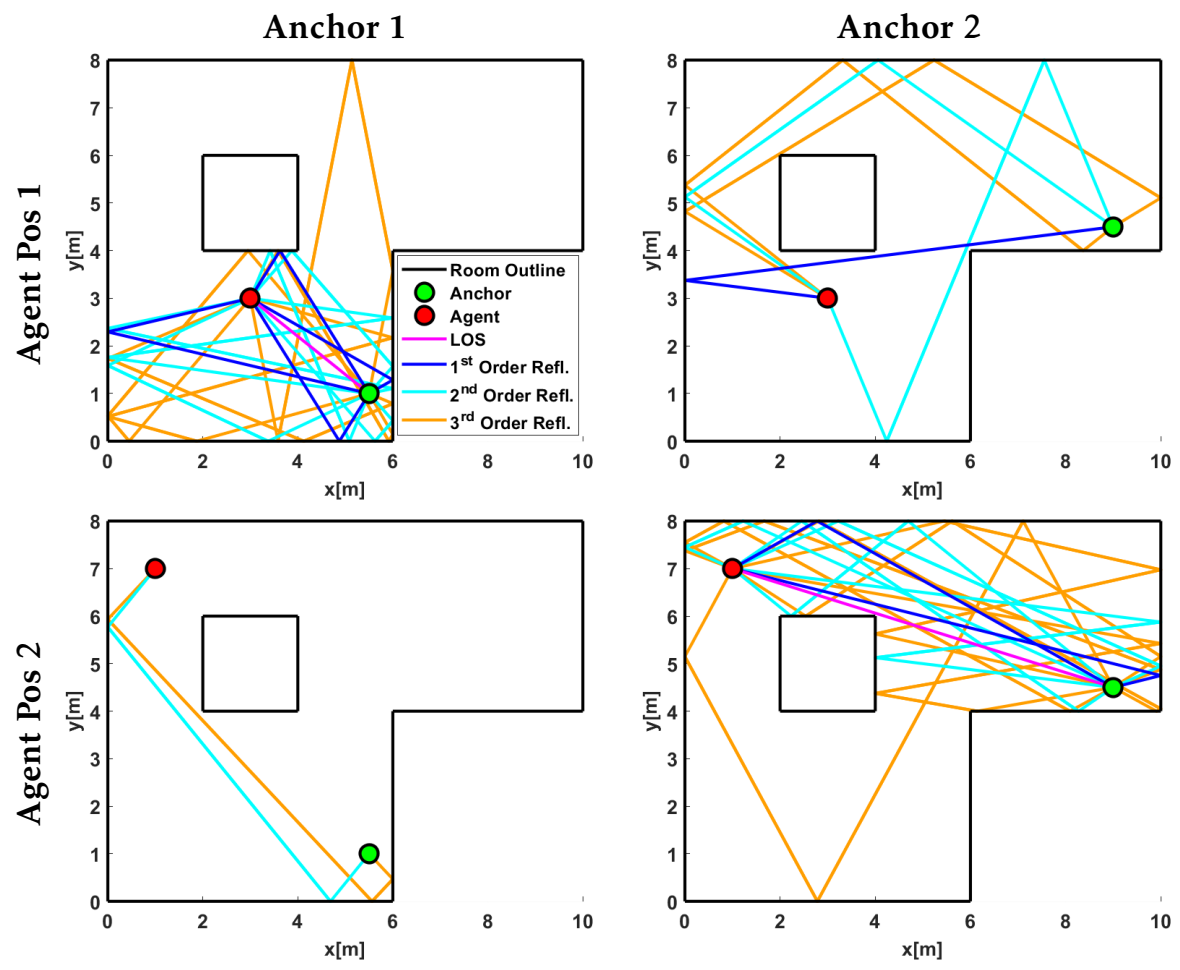

Figure 2.5: The reflected signal paths (MPCs) in the considered scenario. 
Anchor 1

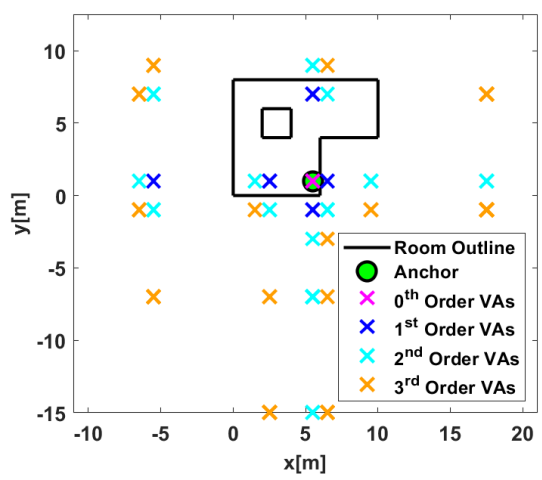

Anchor 2

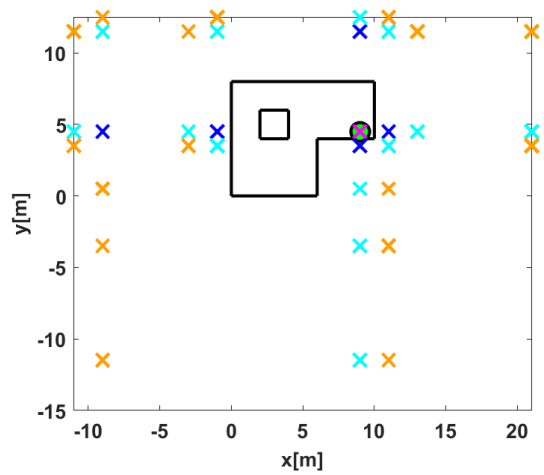

Figure 2.6: All the VAs of both anchors in the considered scenario.

Anchor 1
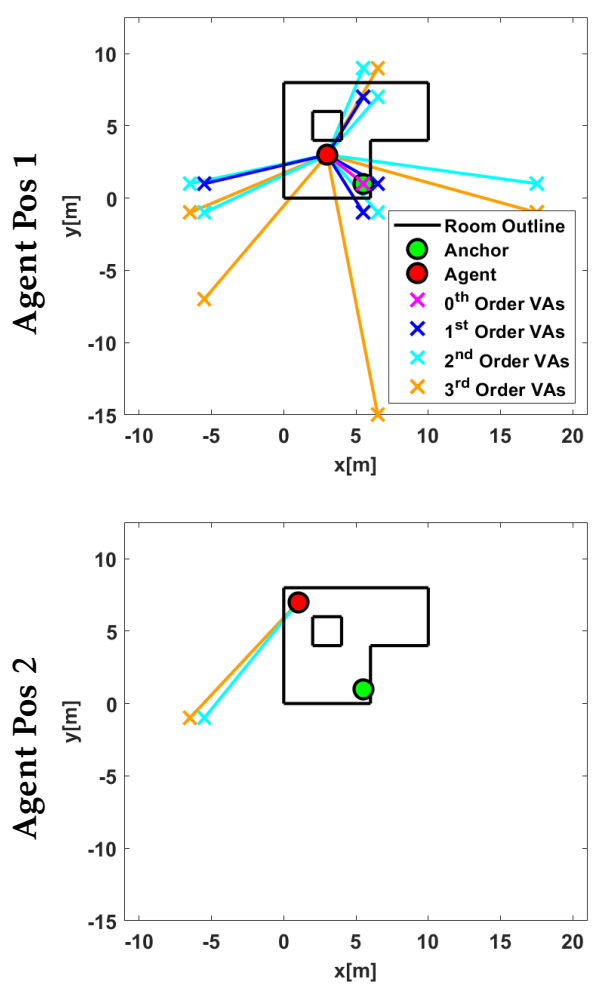

Anchor 2
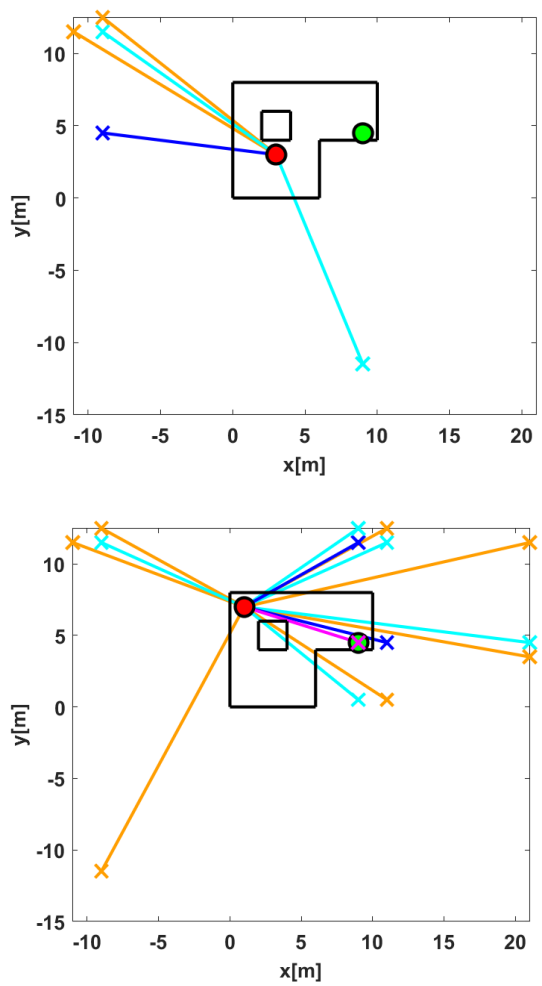

Figure 2.7: The visible VAs corresponding to the MPCs of Figure 2.5. 


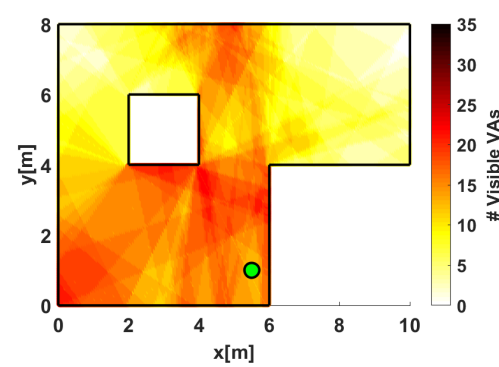

(a) Anchor 1

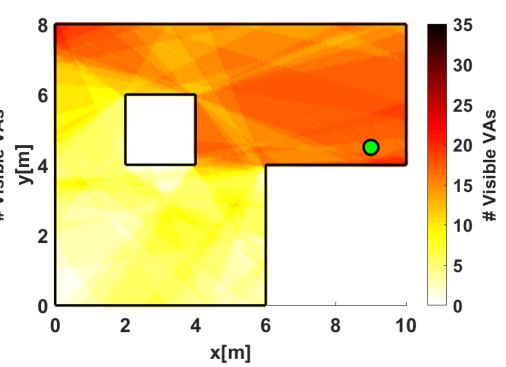

(b) Anchor 2

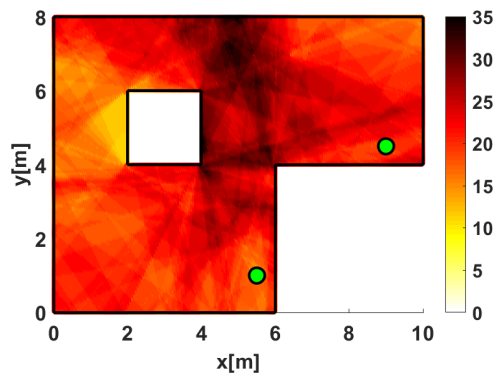

(c) Both Anchors

Figure 2.8: The total number of visible MPCS/VAs at any point in the room considering (a) Anchor 1, (b) Anchor 2 and (c) Both Anchors Jointly.

Anchor 1
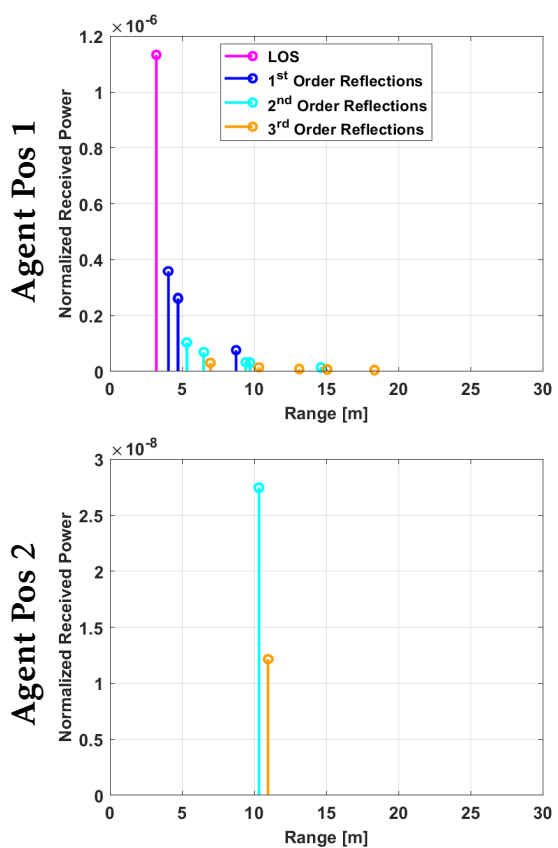

Anchor 2
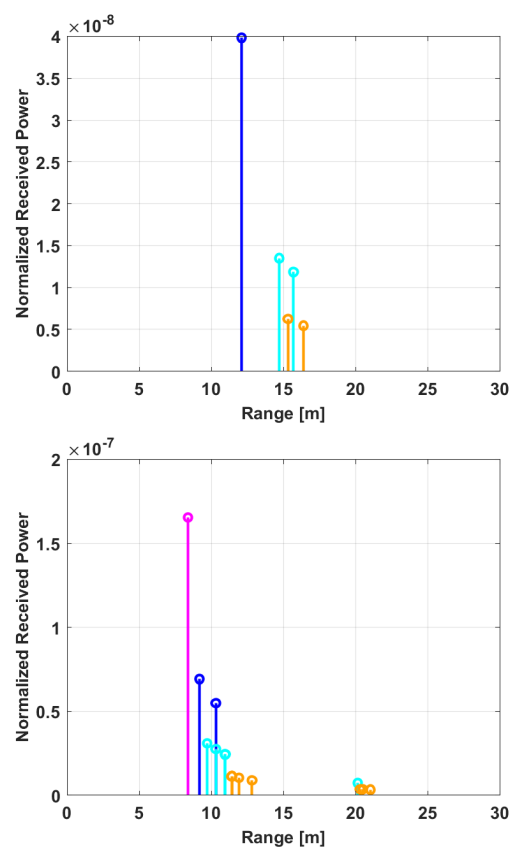

Figure 2.9: The received power versus range for all visible MPCs. 


\section{Example 2.2}

Consider now the circular trajectory of Scenario A depicted in Figure 2.2. The resulting ranges for the respective MPCs are shown in Figure 2.10, whereas the number of visible VAs as well as an indication of the LOS conditions (i.e., if the LOS component is visible or not) are shown in Figure 2.11. What is here denoted as 'Position Index' is nothing but the angle in degrees counted counter-clockwise relative the negative y-axis, where the agent started, as discussed for Figure 2.2.

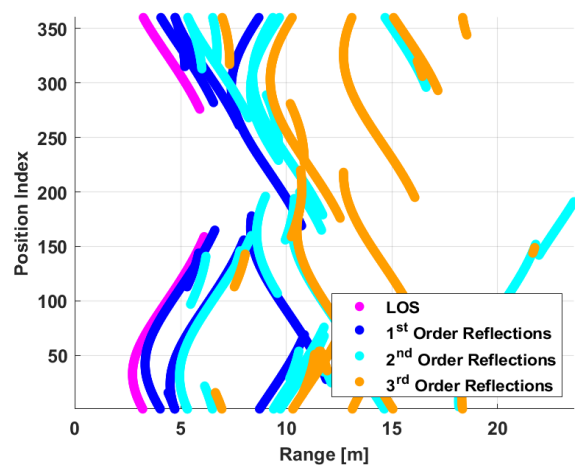

(a) Anchor 1

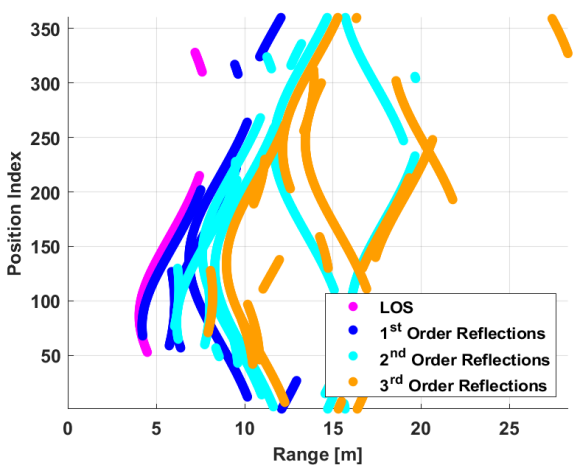

(b) Anchor 2

Figure 2.10: The reflected signal paths (MPCs) over the trajectory.

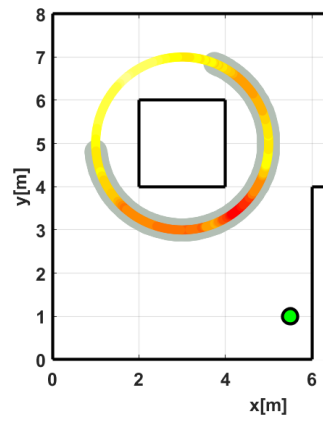

(a) Anchor 1

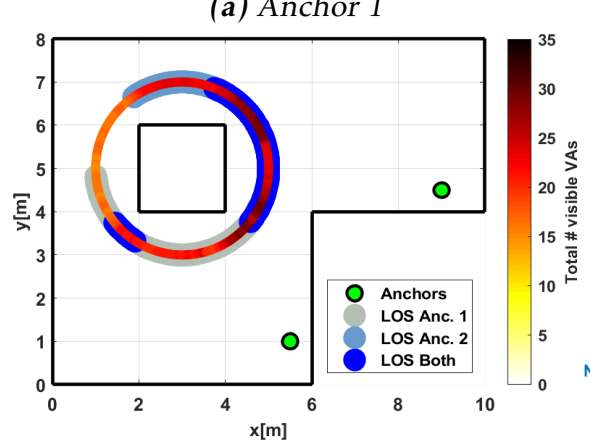

(c) Both Anchors

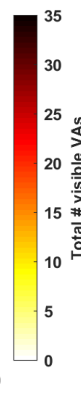

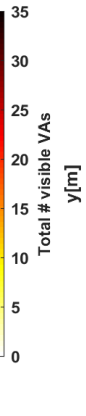

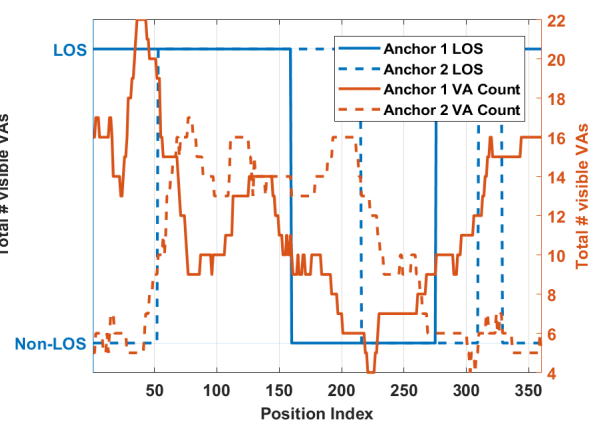

(d) Timeplot

Figure 2.11: The total number of visible MPCS/VAs and LOS conditions. 


\subsection{Signal Model}

In this work, we follow what was done in e.g. $[7,29]$ and consider the transmitted pulse $s(t)$ to be an Ultra-Wideband (UWB) raised cosine pulse:

$$
s(t)=\operatorname{sinc}\left(\frac{t}{T}\right) \frac{\cos \left(\frac{\pi \beta t}{T}\right)}{1-\left(\frac{2 \beta t}{T}\right)^{2}},
$$

where $T$ is the pulse duration and $\beta$ is the roll-off factor. This pulse is normalized to contain unit energy per transmit antenna, i.e.,

$$
\int_{-\infty}^{\infty}|s(t)|^{2} d t=1
$$

The auto-correlation function of $s(t)$ becomes

$$
R_{s}(\tau)=\int_{-\infty}^{\infty} s(t) s(t-\tau) d t=\operatorname{sinc}\left(\frac{\tau}{T}\right) \frac{\cos \left(\frac{\pi \beta \tau}{T}\right)}{1-\left(\frac{2 \beta \tau}{T}\right)^{2}}-\frac{\beta}{4} \operatorname{sinc}\left(\frac{\beta \tau}{T}\right) \frac{\cos \left(\frac{\pi \tau}{T}\right)}{1-\left(\frac{\beta \tau}{T}\right)^{2}} .
$$

\section{Example 2.3}

An example with pulse duration $T=1$ ns and roll-off factor $\beta=0.6$ as per Table 2.1 on page 10 , gives $s(t)$ and $R_{s}(\tau)$ as in Figure 2.12. Also depicted here are the first and second order derivatives of $R_{s}(\tau)$, which are required for the later calculation of the performance bounds in Chapter 6 .

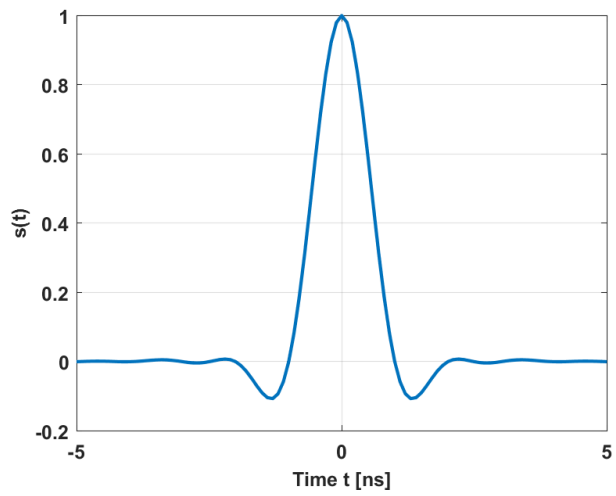

(a) $s(t)$

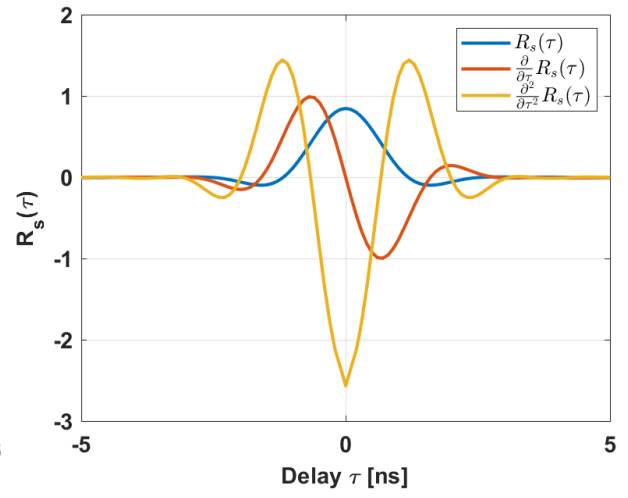

(b) $R_{S}(\tau)$

Figure 2.12: Illustration of (a) $s(t)$ as per (2.27) and (b) $R_{s}(\tau)$ as per (2.29) together with $\frac{\partial}{\partial \tau} R_{s}(\tau)$ and $\frac{\partial^{2}}{\partial^{2} \tau} R_{s}(\tau)$. Parameters are $T=1$ ns and $\beta=0.6$. 


\subsection{Power Delay Profile (PDP)}

The power delay profile (PDP) for the SISO radio channel of $(2.20)$ is here denoted $A_{h}(t, \tau)$. As discussed in [13] and [32], it represents the average power associated with a given multipath delay $\tau$ at a given time $t$ and can be defined as

$$
A_{h}(t, \tau) \delta(\Delta t) \delta(\Delta \tau)=\mathbb{E}\left[h^{*}(t, \tau) h(t+\Delta t, \tau+\Delta \tau)\right]
$$

where it is assumed that the channel is wide-sense stationary (wSS), i.e., that the joint statistics of the channel measured at the two times $t$ and $t+\Delta t$ only depends on the difference $\Delta t$. It is also assumed that any two MPCs with different delays $\tau$ and $\tau+\Delta \tau$ are scattered by different objects, so that the radio channel is subject to the uncorrelated scattering (US) property. Under these assumptions, $h(t, \tau)$ is referred to as a wide-sense stationary uncorrelated scattering (WSSUS) channel for which its PDP $A_{h}(t, \tau)$ in (2.30) can, setting $\Delta t=\Delta \tau=0$, be approximated as

$$
\begin{aligned}
& A_{h}(t, \tau) \stackrel{(1)}{=} \mathbb{E}\left[\left(\sum_{k=1}^{K} \alpha^{(k)} \delta\left(t-\tau^{(k)}\right)+v(t)\right)^{*}\left(\sum_{k=1}^{K} \alpha^{(k)} \delta\left(t-\tau^{(k)}\right)+v(t)\right]\right. \\
& =\mathbb{E}\left[\sum_{k=1}^{K} \sum_{k^{\prime}=1}^{K}\left[\alpha^{(k)}\right]^{*} \alpha^{\left(k^{\prime}\right)} \delta\left(t-\tau^{(k)}\right) \delta\left(t-\tau^{\left(k^{\prime}\right)}\right)\right. \\
& \left.+v^{*}(t) \sum_{k=1}^{K} \alpha^{(k)} \delta\left(t-\tau^{(k)}\right)+v(t) \sum_{k=1}^{K}\left[\alpha^{(k)}\right]^{*} \delta\left(t-\tau^{(k)}\right)+v^{*}(t) v(t)\right] \\
& \stackrel{(2)}{=} \sum_{k=1}^{K} \mathbb{E}\left[\left|\alpha^{(k)}\right|^{2}\right] \delta\left(t-\tau^{(k)}\right)+\mathbb{E}\left[v^{*}(t) v(t)\right] \stackrel{(3)}{=} \sum_{k=1}^{K}\left|\alpha^{(k)}\right|^{2} \delta\left(t-\tau^{(k)}\right)+S_{v}(\tau)
\end{aligned}
$$

where at $\stackrel{(1)}{=}(2.14)$ has been used, at $\stackrel{(2)}{=}$ that $v(t)$ and $\alpha^{(k)} \delta\left(t-\tau^{(k)}\right)$ per definition are uncorrelated for all times $t$ and all MPCs $k \in \mathcal{K}$. At $\stackrel{(3)}{=}$ it has been assumed that the environment is static so that the $\left\{\alpha^{(k)}\right\}$ s become deterministic. Also used at this point is the PDP of the DM defined as $S_{v}(\tau)$ previously in Section 2.2.

The expression (2.31) cannot be measured in practice since both the parameters $\left\{\alpha^{(k)}\right\},\left\{\tau^{(k)}\right\}$ of the $K$ deterministic MPCs as well as the PDP for the DM $S_{v}(\tau)$ are unknown. What can be done is however as in [27] to calculate

$$
\begin{aligned}
& \mathbb{E}\left[|r(t)|^{2}\right]=\mathbb{E}\left[r^{*}(t) r(t)\right] \\
& =\mathbb{E}\left[\left(\sum_{k=1}^{K} \alpha^{(k)} s\left(t-\tau^{(k)}\right)+v \star s(t)+w(t)\right)^{*}\left(\sum_{k=1}^{K} \alpha^{(k)} s\left(t-\tau^{(k)}\right)+v \star s(t)+w(t)\right)\right] \\
& \stackrel{(1)}{=} \mathbb{E}\left[\sum_{k=1}^{K} \sum_{k^{\prime}=1}^{K}\left[\alpha^{(k)}\right]^{*} \alpha^{\left(k^{\prime}\right)} s\left(t-\tau^{(k)}\right) s\left(t-\tau^{\left(k^{\prime}\right)}\right)\right]+\mathbb{E}\left[|v \star s(t)|^{2}\right]+\mathbb{E}\left[|w(t)|^{2}\right] \\
& \stackrel{(2)}{=} \sum_{k=1}^{K}\left|\alpha^{(k)}\right|^{2}\left|s\left(t-\tau^{(k)}\right)\right|^{2}+\mathbb{E}\left[|v \star s(t)|^{2}\right]+\mathbb{E}\left[|w(t)|^{2}\right] \stackrel{(3)}{=} \sum_{k=1}^{K}\left|\alpha^{(k)}\right|^{2}\left|s\left(t-\tau^{(k)}\right)\right|^{2}+e(t)
\end{aligned}
$$


where at $\stackrel{(1)}{=}$ all cross-terms are eliminated because of the assumptions of uncorrelated scattering and noise and $\stackrel{(2)}{=}$ (again) that the environment is static. Finally, at $\stackrel{(3)}{=}$, the noise term $e(t)$ is introduced which caters for the expected power of both DM as well as AWGN measurement noise. Thereafter, the PDP can be approximated by averaging over $M$ measurements $\left\{r^{(m)}\right\}$ collected over a small enough spatial region around the receiver

$$
A_{h}(t, \tau) \approx \frac{1}{M} \sum_{m=1}^{M}\left|r^{(m)}(t)\right|^{2} .
$$

\section{Example 2.4}

We continue from Example 2.1 and look in particular at the agent at $\mathbf{x}=[3,3]^{T}$ with the parameter settings as per Table 2.1 on page 10 . Hence with carrier frequency $f_{c}=7 \mathrm{GHz}$, UWB pulse duration $T=1 \mathrm{~ns}$ and roll-off factor $\beta=0.6$. The DM parameters are $\gamma_{\text {rise }}=5 \mathrm{~ns}, \gamma_{1}=20 \mathrm{~ns}, \chi=0.98$ and $\Omega_{1}=\frac{1}{10}\left(\frac{c}{4 \pi f_{c}}\right)^{2}$. The AWGN power density is a modest $N_{0}=10^{-8}$, i.e., this is the high SNR scenario. We assume here (and in throughout this thesis) 100×oversampling, whereby the sampling time is set to $T_{s}=T / 100=10 \mathrm{ps}$.

Letting $m \in\{1,2\}$ denote the respective anchor, we plot in Figures 2.13a and $2.13 \mathrm{~b}$ the squared MPC amplitudes $\left\{\left|\alpha_{m}^{(k)}\right|^{2}\right\}$ provided by the environmental/geometric model discussed in Section 2.3 (again, we consider a maximum of 3 reflections) together with the power density (PSD) of both the DM $v_{m}(t)$ as well as the AWGN noise $w(t)$. Clearly the deterministic MPCs have lower power, and hence the noise is more pronounced, for the second anchor. This is not surprising given that it is not only further away but also that the LOS component is blocked in this case. The number above each bar indicates how many MPC that have a similar propagation delay and cannot be distinguished the given sample rate, i.e., the number of MPCs for which $\left|\tau_{m}^{(k)}-\tau_{m}^{\left(k^{\prime}\right)}\right|<T_{s}$ for $k \neq k^{\prime}$, something which is further discussed in Section 6.1 of Chapter 6.

In Figures 2.13c and 2.13d we plot the power of the received signal (2.21) with respect to the respective anchor(s). Here we clearly see the effect of adding the DM and/or AWGN components to the ideal signal. In particular for the second anchor, the contributions from the DM and AWGN are significant. Clearly, it appears that it can be troublesome to estimate the MPCs based on the received signal, even though the AWGN noise is rather low in this scenario. This topic is discussed further in more detail later in Section 3.2.

Finally, intended only for illustrative purposes, in Figures 2.13e and 2.13f, we show the received signal $r_{m}(t)$ with DM and AWGN (i.e., same as in Figures 2.13c and $2.13 \mathrm{~d}$ ), together with what would have been the case had it been sampled with a lower sampling rate equal to that of the UWB pulse duration, i.e., $T_{S}=$ $T=1$ ns. Clearly, this makes it even more problematic due to aliasing effects etc. Throughout the rest of this thesis, we will therefore stick to the shorter sampling time of $T_{s}=10 \mathrm{ps}$, since a detailed discussion of sampling and related problems are considered outside the scope of this thesis. 


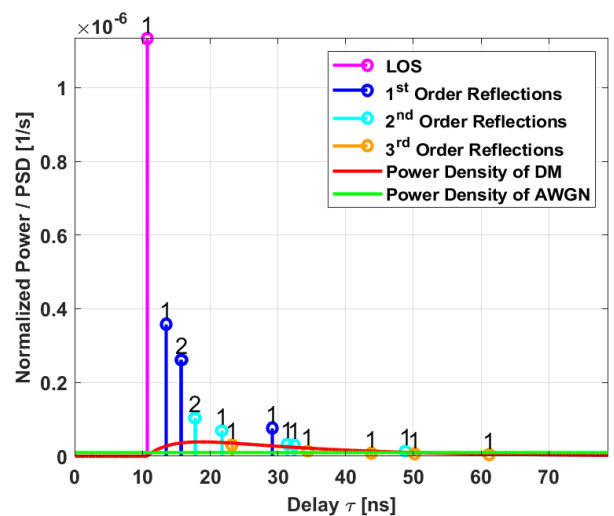

(a) $h_{1}(t)$

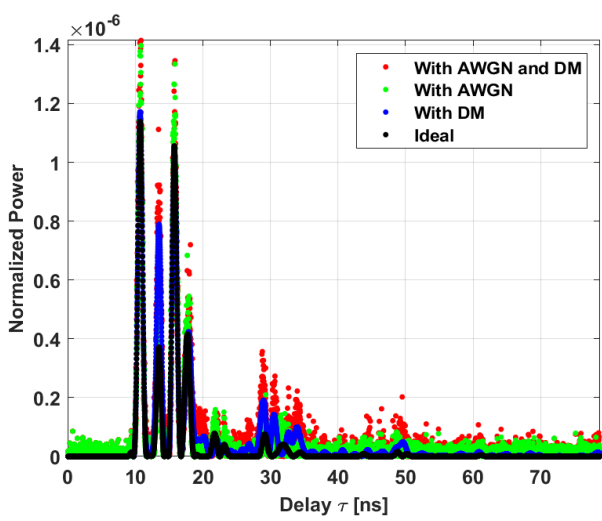

(c) $r_{1}(t)$

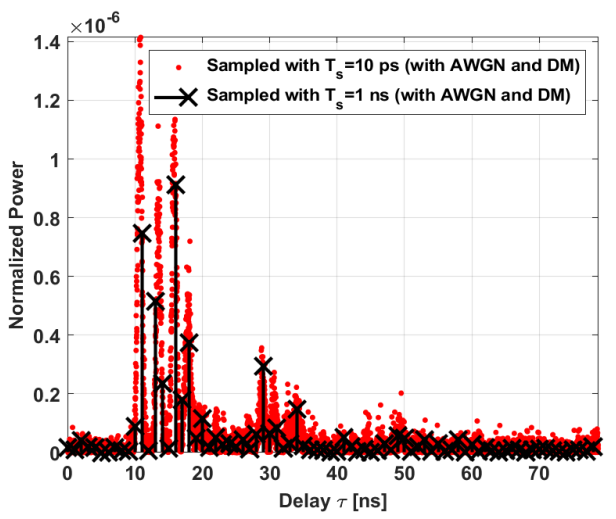

(e) Sampled $r_{1}(t)$

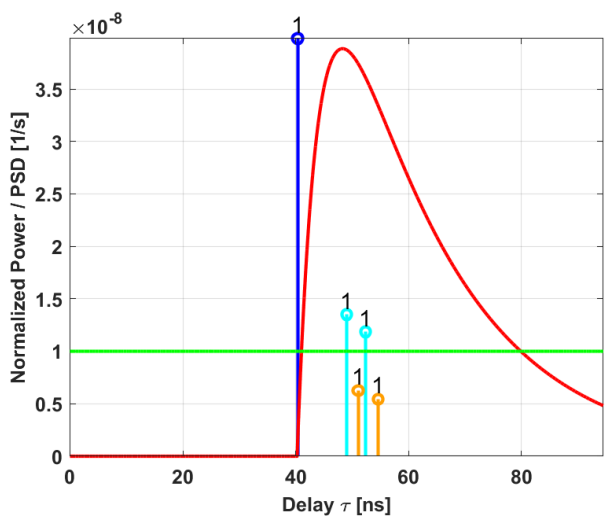

(b) $h_{2}(t)$

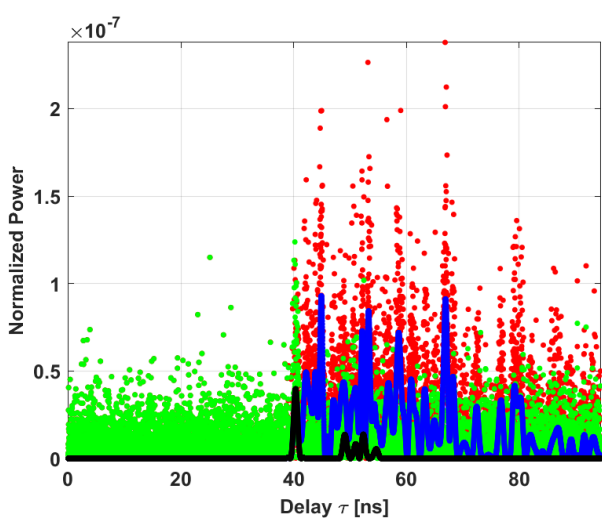

(d) $r_{2}(t)$

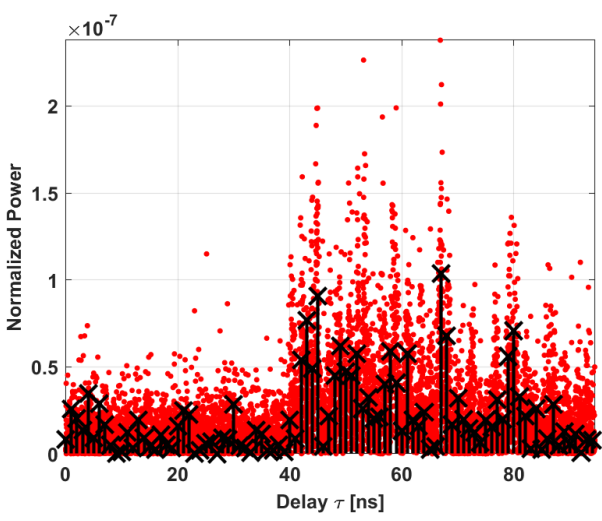

(f) Sampled $r_{2}(t)$

Figure 2.13: The channel impulse response $h_{m}(t)$, the received signal with the addition of the AWGN and/or DM noise components as well as differently sampled versions of the received signal $r_{m}(t)$ for the considered scenario. 


\section{Example 2.5}

We continue with the same settings as in Example 2.4, but now for the trajectory of Scenario A we visited in Example 2.2. This gives us results as per Figure 2.14 below in the high SNR case when the AWGN noise is set to $N_{0}=10^{-8}$. Here we clearly see the dominance of, the DM noise during the non-LOS periods for the respective anchor. Again, the 'Position Index' is the angle in degrees counted counter-clockwise relative the negative y-axis.
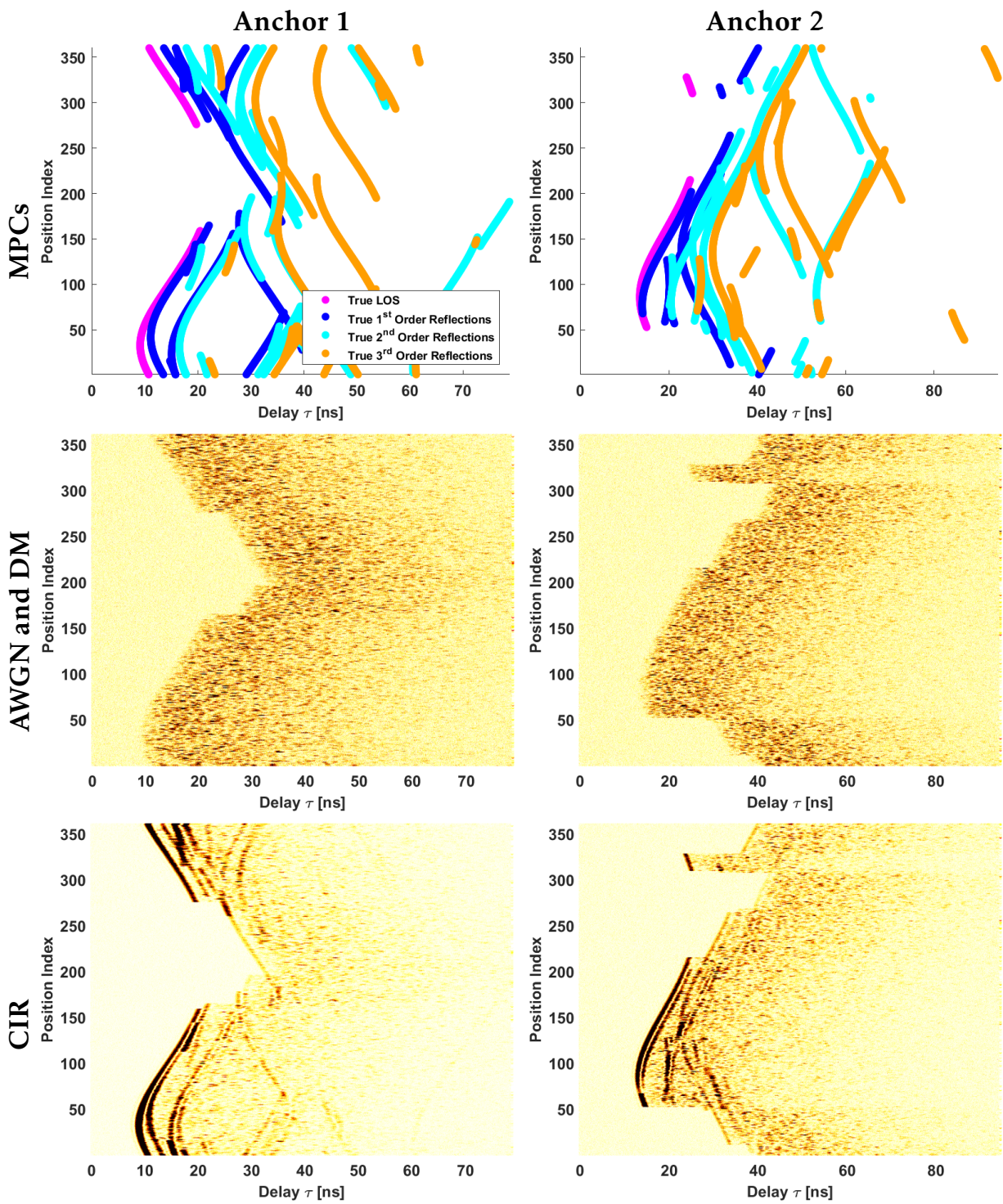

Figure 2.14: The true MPC, the addition of the AWGN and DM as well as the resulting CIR for the trajectory example of Scenario A with high SNR $\left(N_{0}=10^{-8}\right)$. 
In addition, in Figure 2.15 we also show the low SNR case when the AWGN noise is set to $N_{0}=10^{-6}$. The noise is significant and the contribution from the deterministic MPCs is barely distinguishable from the noise.

Anchor 1
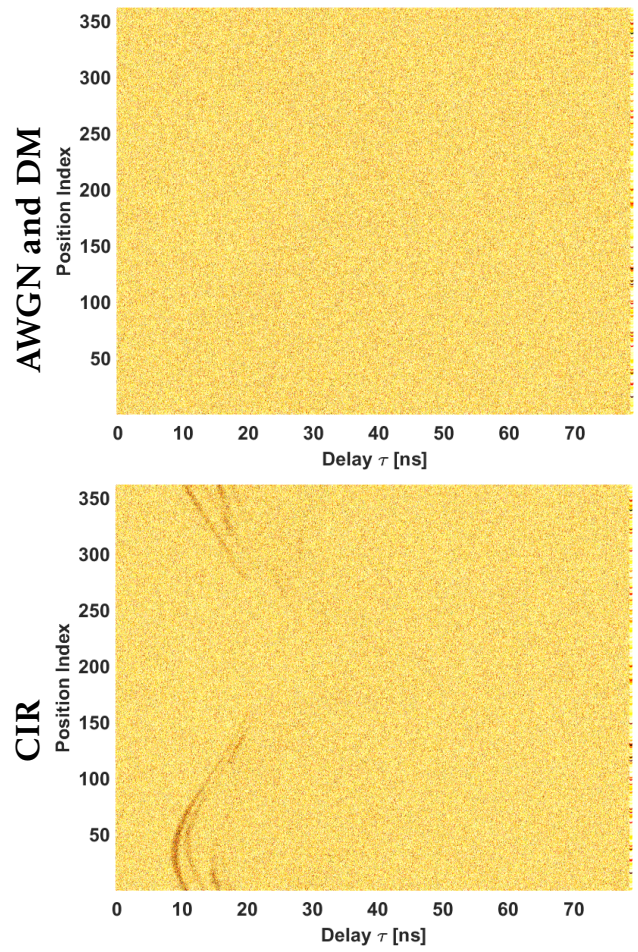

Anchor 2
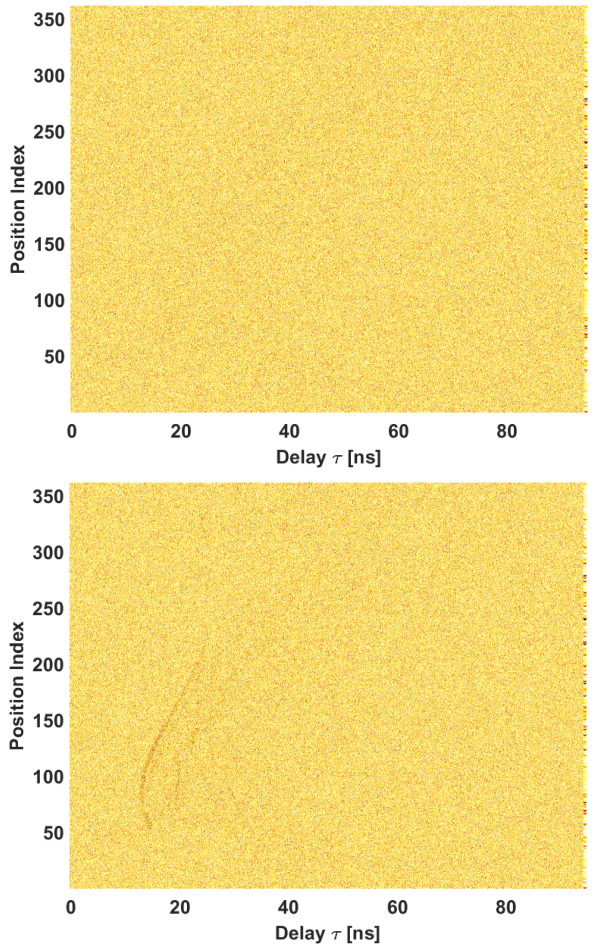

Figure 2.15: The addition of the AWGN and DM as well as the resulting CIR for the trajectory example of Scenario A with low SNR $\left(N_{0}=10^{-6}\right)$. 


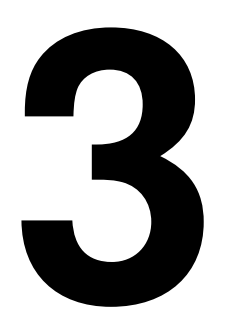

\section{Estimation and Measurements}

In this thesis, we consider two distinct estimation problems: The first is the estimation of the MPCs, i.e., the channel parameters $\alpha, \tau$ introduced in Chapter 2, and the second is the estimation of the unknown agent position $\mathbf{x}$. In Section 3.2 we discuss the former and the latter in Section 3.4. Before that, a brief introduction to the estimation methods used in this thesis is given in Section 3.1. In Section 3.3, we define the pseudo-range measurements used in the subsequent chapters of this thesis, which are based on the now estimated MPCs.

\subsection{Estimation Background}

This section gives a brief introduction to the estimation methods used in this thesis and follows rather closely what is described in e.g. [20], [45], [16] and [42].

\subsubsection{Parameter Estimation}

One way to describe a random measurement vector $\mathbf{z}$ which depends on a parameter vector $\theta$ is through the likelihood function

$$
p(\mathbf{z} \mid \theta)
$$

for which, in the special case of a general linear model, we have the relationship

$$
\mathbf{z}=\mathbf{H} \boldsymbol{\theta}+\mathbf{n}
$$

where $\mathbf{n}$ is noise. This noise is, in typical cases, Gaussian with zero-mean and a covariance matrix $\mathbf{C}$, i.e., $\mathbf{n} \sim \mathcal{N}(\mathbf{0}, \mathbf{C})$, which then gives

$$
p(\mathbf{z} \mid \boldsymbol{\theta})=\mathcal{N}(\mathbf{H} \boldsymbol{\theta}, \mathbf{C}) .
$$


Bayes theorem (see [42]) gives that the posterior probability distribution of $\mathbf{z}$ given the parameter vector $\theta$ is

$$
p(\boldsymbol{\theta} \mid \mathbf{z})=\frac{p(\mathbf{z} \mid \boldsymbol{\theta}) p(\boldsymbol{\theta})}{p(\mathbf{z})}=\frac{p(\mathbf{z} \mid \boldsymbol{\theta}) p(\boldsymbol{\theta})}{\int p(\mathbf{z} \mid \boldsymbol{\theta}) p(\boldsymbol{\theta}) d \boldsymbol{\theta}}
$$

where $p(\mathbf{z} \mid \theta)$ is the likelihood and $p(\theta)$ is the prior distribution, respectively.

To estimate the unknown parameter vector $\theta$, one approach is to form the maximum a posteriori (MAP) estimator

$$
\hat{\boldsymbol{\theta}}_{\mathrm{MAP}}=\arg \max _{\theta} p(\boldsymbol{\theta} \mid \mathbf{z})=\arg \max _{\theta} p(\mathbf{z} \mid \boldsymbol{\theta}) p(\boldsymbol{\theta})=\arg \max _{\theta}[\ln p(\mathbf{z} \mid \boldsymbol{\theta})+\ln p(\boldsymbol{\theta})]
$$

which in case of a non-informative prior, i.e., when $p(\theta)$ is constant, collapses to the special case of the maximum likelihood (ML) estimator given by

$$
\hat{\theta}_{\mathrm{ML}}=\arg \max _{\theta} p(\mathbf{z} \mid \boldsymbol{\theta})=\arg \max _{\theta} \ln p(\mathbf{z} \mid \boldsymbol{\theta}) .
$$

Now, for the special case of the general linear model in (3.2), we get the best linear unbiased (BLUE) estimator

$$
\hat{\theta}_{\mathrm{BLUE}}=\left(\mathbf{H}^{T} \mathbf{C}^{-1} \mathbf{H}\right)^{-1} \mathbf{H}^{T} \mathbf{C}^{-1} \mathbf{z}
$$

with covariance

$$
\mathbf{C}_{\hat{\theta}}=\left(\mathbf{H}^{T} \mathbf{C}^{-1} \mathbf{H}\right)^{-1}
$$

which, in case the noise is also Gaussian as per (3.3) not only coincides with the ML estimator of (3.6), i.e., $\hat{\theta}_{\mathrm{ML}}=\hat{\theta}_{\mathrm{BLUE}}$, but then also is the minimum variance unbiased (MVU) estimator, see e.g. [16].

\subsubsection{State Estimation}

In this section a temporal aspect to the estimation problem is added. More precisely, assume that we have a time-series of noisy observations $\mathbf{z}_{t}$ for $t=1, \ldots, T$ and want to infer the hidden state variables $\mathbf{x}_{t}$. Assume further that the underlying model is a state-space model (see e.g. [26], [42] and [16]):

$$
\begin{aligned}
\mathbf{x}_{0} & \sim p\left(\mathbf{x}_{0}\right) \\
\mathbf{x}_{t} & \sim p\left(\mathbf{x}_{t} \mid \mathbf{x}_{t-1}\right) \\
\mathbf{z}_{t} & \sim p\left(\mathbf{z}_{t} \mid \mathbf{x}_{t}\right)
\end{aligned}
$$

where $p\left(\mathbf{x}_{0}\right)$ is the prior distribution of $\mathbf{x}_{0}$, where $p\left(\mathbf{x}_{t} \mid \mathbf{x}_{t-1}\right)$ is the dynamic model and $p\left(\mathbf{z}_{t} \mid \mathbf{x}_{t}\right)$ is the likelihood specifying the measurement model. 
What is searched for is hence an estimate of the hidden state variables $\mathbf{x}_{t}$ for $t=1, \ldots, T$, which can e.g. be accomplished by the means of a particle filter (PF) as discussed in e.g. [42], [15] and [16]. The particle filter algorithm used in this thesis is given in Algorithm 1 below, but for more details and derivation, as well as alternative approaches, we refer to see the above listed references.

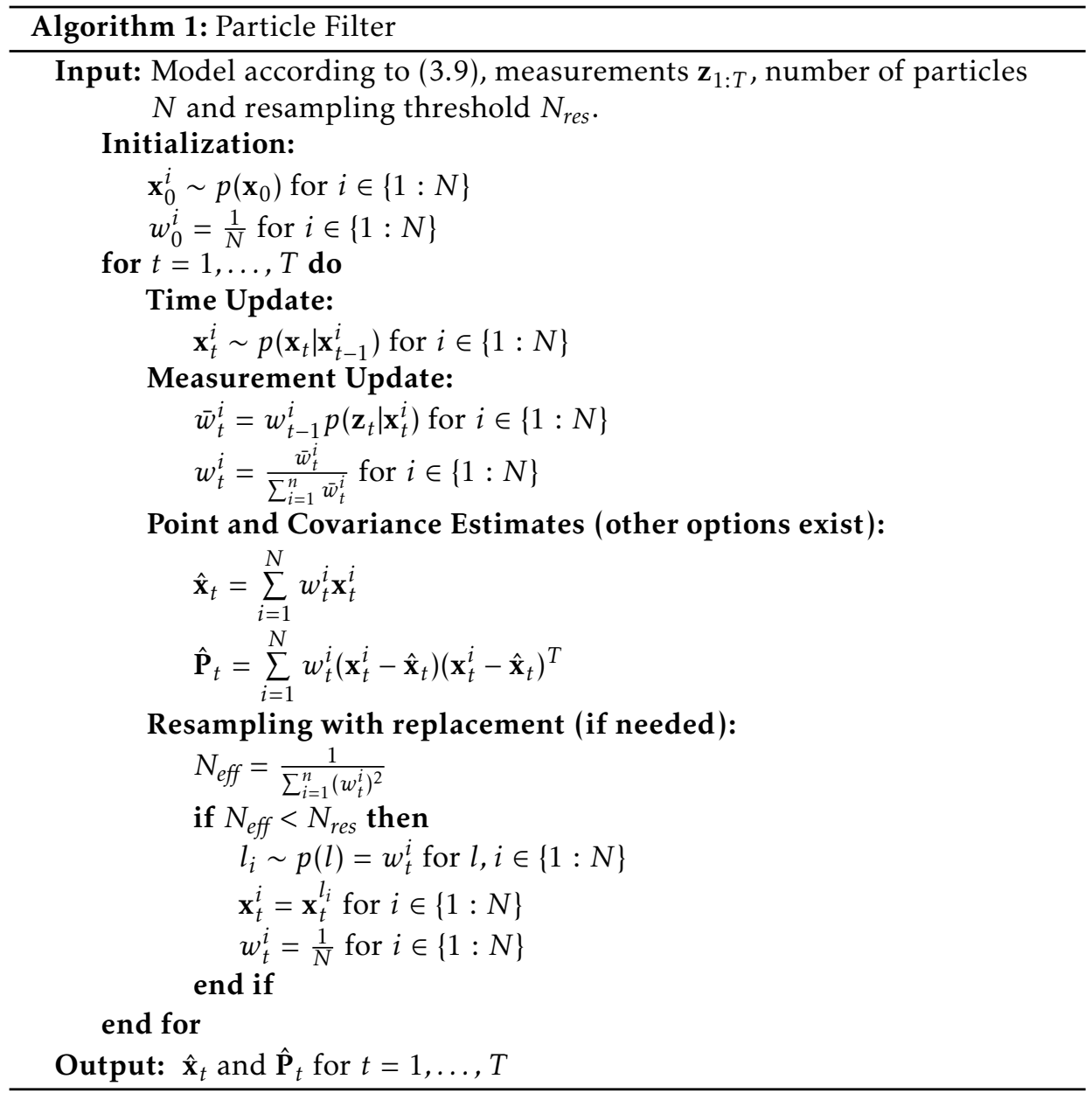




\subsection{Estimation of Channel Parameters / MPCs}

This section discusses the estimation of the channel parameters $\alpha$, $\tau$ introduced in Chapter 2. It follows the procedure proposed in [25] and [31].

Using an iterative least-squares approach, the delay for MPC $k=1, \ldots, K_{m}$ in the channel between the agent and anchor $m=1, \ldots, M$ can be estimated as

$$
\hat{\tau}_{m}^{(k)}=\arg \min _{\tau} \int_{t=0}^{T}\left|r_{m}(t)-\hat{r}_{m}^{(k-1)}(t)-\hat{\alpha}(\tau) s(t-\tau)\right|^{2} d t
$$

in which the template signal $\hat{r}_{m}^{(k)}(t)$ is defined as

$$
\hat{r}_{m}^{(k)}(t)=\sum_{k^{\prime}=1}^{k} \hat{\alpha}_{m}^{\left(k^{\prime}\right)} s\left(t-\hat{\tau}_{m}^{\left(k^{\prime}\right)}\right)
$$

where the path amplitudes are estimated by a projection of the received signal $\hat{r}_{m}^{(k)}(t)$ onto the unit energy transmitted pulse $s(t)$ :

$$
\hat{\alpha}(\tau)=\int_{t=0}^{T}\left[r_{m}(t)\right]^{*} s(t-\tau) d t \quad \text { and } \quad \hat{\alpha}_{m}^{(k)}=\hat{\alpha}\left(\hat{\tau}_{m}^{(k)}\right) .
$$

The whole procedure is summarized in Algorithm 2. Here, a parameter $K_{M A X}$ is introduced which should (if computationally feasible) be chosen according to the expected number of specular paths, i.e., $K_{m}$, as far as possible.

It should be noted that, if assuming separable MPCs and further the noise to be Gaussian and additionally choosing $K_{M A X}=\left|\mathcal{K}_{m}\right|$, Algorithm 2 corresponds to a maximum likelihood (ML) estimate of all the deterministic MPCs $k \in \mathcal{K}_{m}$ as discussed in Section 3.1.1.

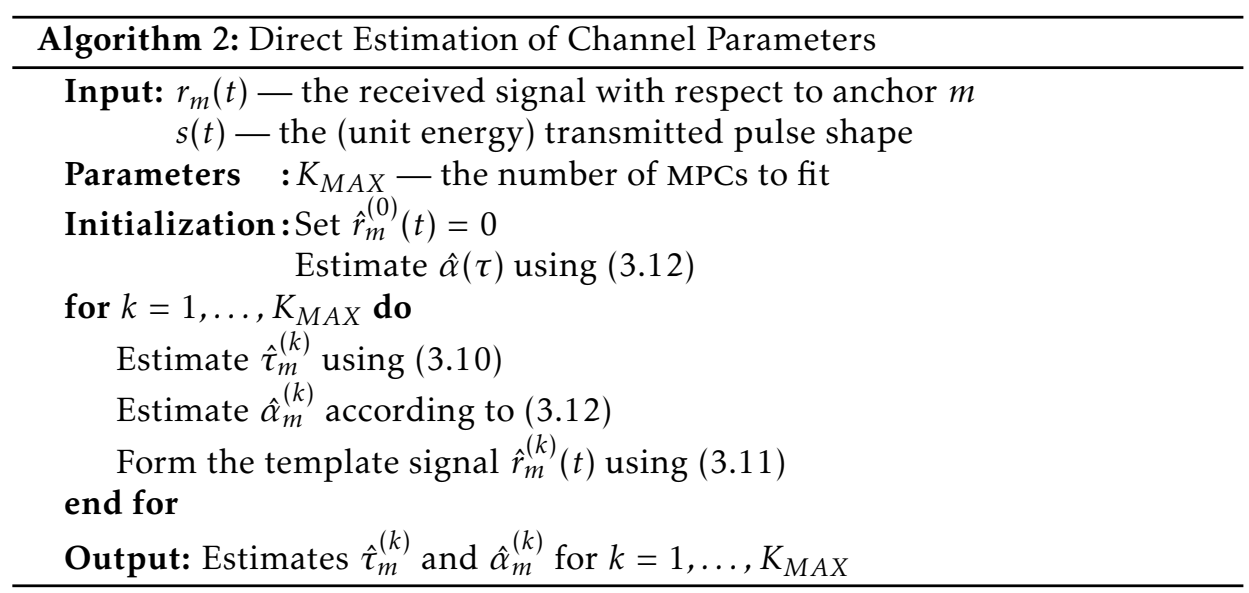




\section{Example 3.1}

We continuing the trajectory of Scenario A from Example 2.5 and apply the procedure of Algorithm 2 with $K_{M A X}=5$. This gives us results as in Figure 3.1 for the high SNR $\left(N_{0}=10^{-8}\right)$ case, when plotted superimposed on the actual deterministic MPCs as well as on the measured CIR. The corresponding plots for the low SNR $\left(N_{0}=10^{-6}\right)$ case are shown in Figure 3.2. Again, the 'Position Index' is the angle in degrees counted counter-clockwise relative the negative y-axis.

From these plots, we see that the estimated MPCs rather well approximate the contribution from, at least the strongest, deterministic MPCs in both cases, but that we get some errors especially during non-LOS conditions, when the DM noise is significant. Further, in the low SNR case, we see the effect of misinterpreting the noisy measurements as false MPCs. This topic is discussed in greater detail later in Chapter 4.

Anchor 1
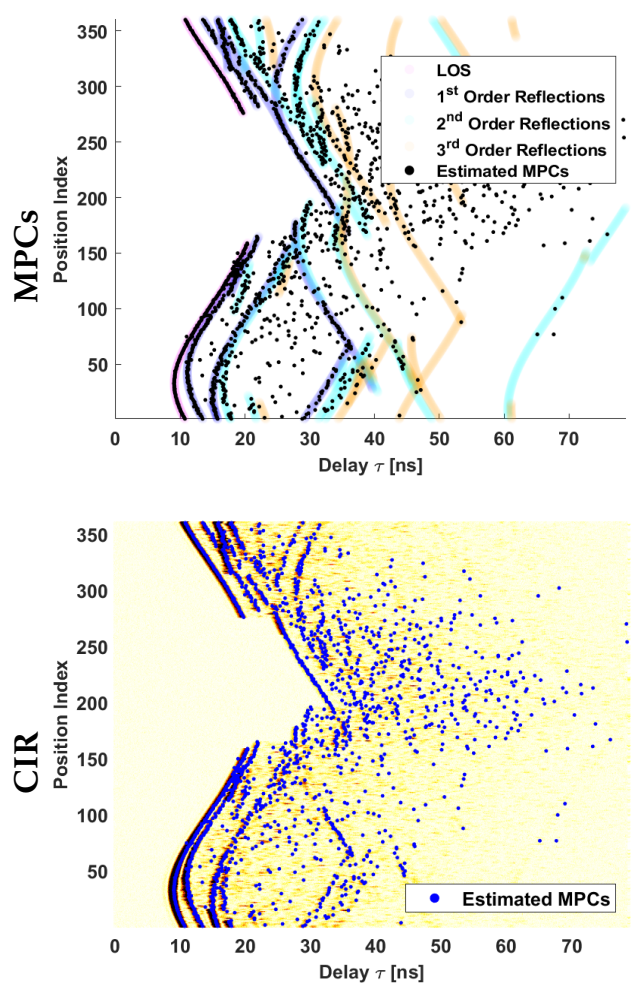

Anchor 2
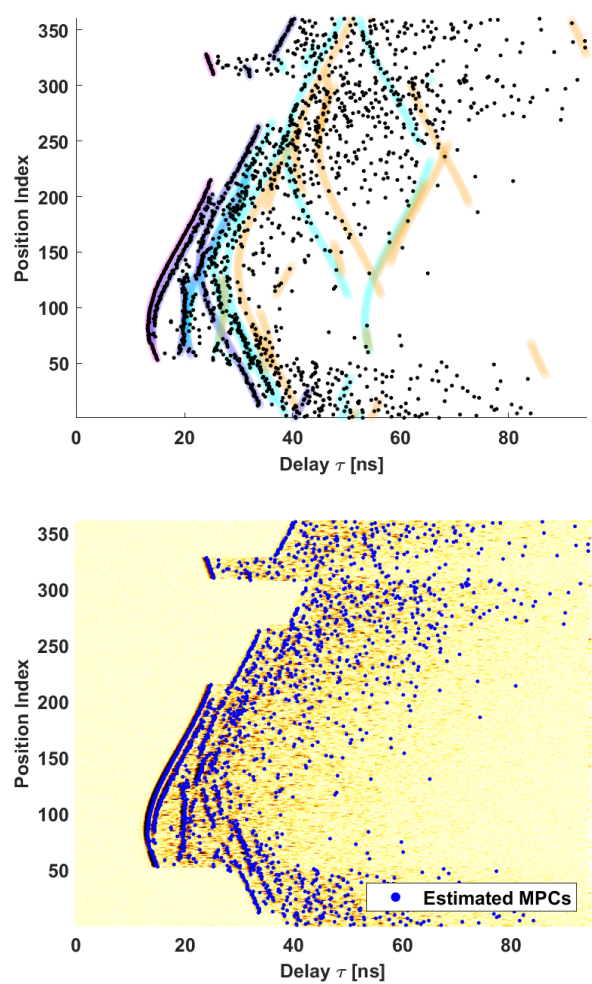

Figure 3.1: The estimated MPCs superimposed on the actual MPCs (top) as well as on the CIR (bottom) for the high SNR $\left(N_{0}=10^{-8}\right)$ trajectory case of Scenario A. 

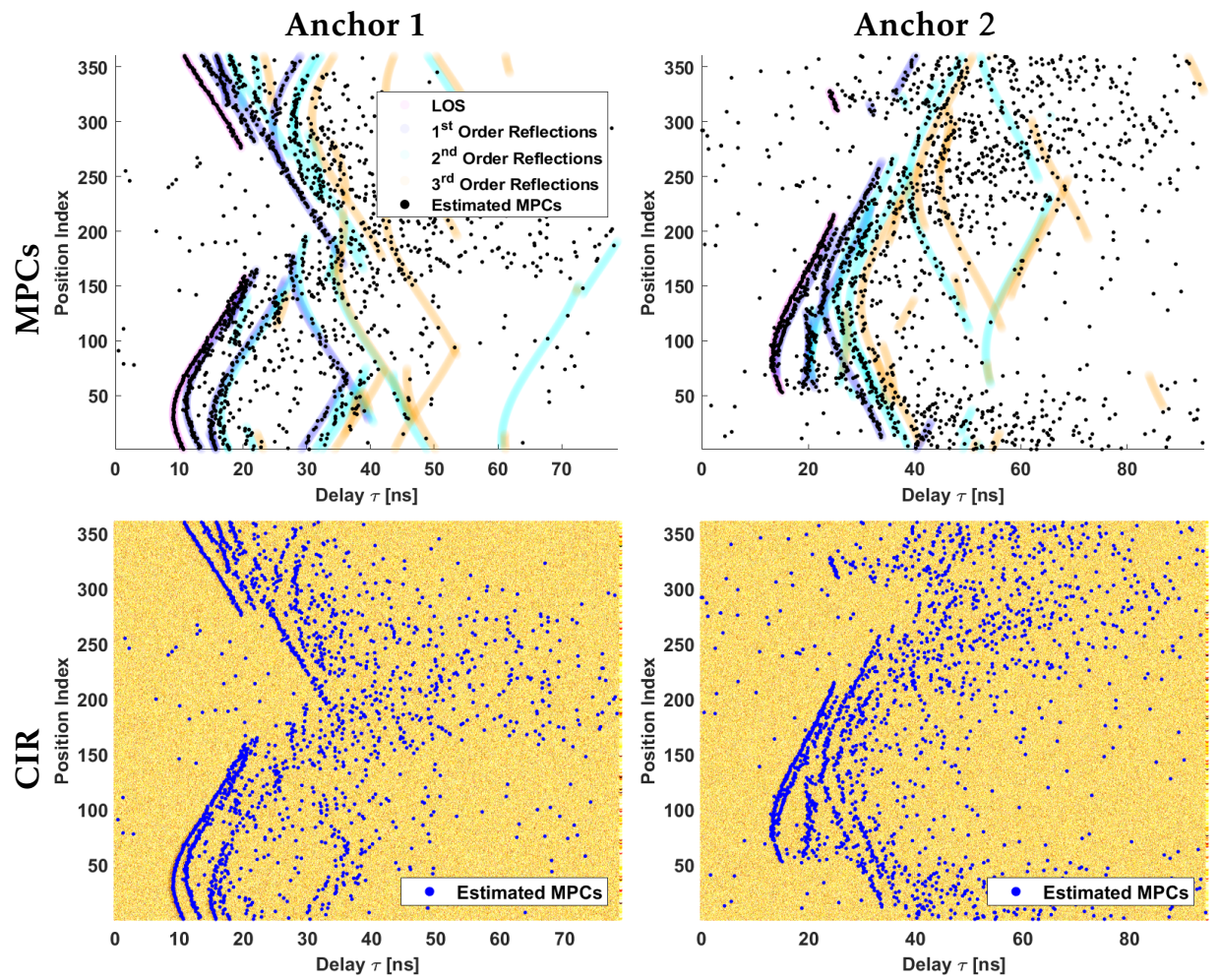

Figure 3.2: Same as Figure 3.1, but for the low $\operatorname{SNR}\left(N_{0}=10^{-6}\right)$ case.

\subsection{Full Pseudo-Range Measurements}

Having established $\hat{\tau}_{m}^{(k)}$, i.e., an estimate of the time-delay $\tau_{m}^{(k)}$ of MPC $k$ in the radio channel between the agent and anchor $m$ as discussed in Section 3.2, we can as per (2.23) express this as the pseudo-range measurement $d_{m}^{(k)}=c \hat{\tau}_{m}^{(k)}$ where $c$ is the speed of light. We assume for simplicity that the therewith associated estimation error is zero-mean Gaussian with a given standard deviation.

The term full in the heading of this section is to indicate that we discuss measurements of all estimated/available MPCs, in contrast to the later Chapter 4 in which we focus only on the measurements and estimation relating to the DP/LOS MPC representing the shortest Euclidean distance between the anchor and agent.

\subsubsection{Uni-Directional}

The pseudo-range measurement between an agent located at $\mathbf{x}$ with respect to MPC/VA $k$ of anchor $m$ (with the corresponding VA located at $\mathbf{p}_{m}^{(k)}$ ) becomes 


$$
y_{m}^{(k)}=\underbrace{\left\|\mathbf{x}-\mathbf{p}_{m}^{(k)}\right\|_{2}}_{\triangleq d_{m}^{(k)}}+e_{m}^{(k)} \text { where } e_{m}^{(k)} \sim \mathcal{N}\left(0, \sigma_{m, k}^{2}\right),
$$

where thus $d_{m}^{(k)} \triangleq\left\|\mathbf{x}-\mathbf{p}_{m}^{(k)}\right\|_{2}$ is the Euclidean distance between the agent and considered VA and where $\sigma_{m, k}$ is the standard deviation of the therewith associated error assumed to be derived from a Gaussian distribution with zero-mean. This means that its probability density function (PDF) is given by

$$
p\left(y_{m}^{(k)} \mid \mathbf{x}, \mathbf{p}_{m}^{(k)}\right)=\mathcal{N}\left(\left\|\mathbf{x}-\mathbf{p}_{m}^{(k)}\right\|_{2}, \sigma_{m, k}^{2}\right)=\frac{1}{\sqrt{2 \pi \sigma_{m, k}^{2}}} \exp \frac{-\left(y_{m}^{(k)}-\left\|\mathbf{x}-\mathbf{p}_{m}^{(k)}\right\|_{2}\right)^{2}}{2 \sigma_{m, k}^{2}}
$$

for which the negative logarithm, which will be used later, is given by

$$
-\ln p\left(y_{m}^{(k)} \mid \mathbf{x}, \mathbf{p}_{m}^{(k)}\right)=\frac{1}{2} \ln 2 \pi \sigma_{m, k}^{2}+\frac{\left(y_{m}^{(k)}-\left\|\mathbf{x}-\mathbf{p}_{m}^{(k)}\right\|_{2}\right)^{2}}{2 \sigma_{m, k}^{2}} .
$$

Assuming that we have $N_{m}$ measurements with respect to anchor $m$, we can define a vector containing all these measurements as

$$
\mathbf{y}_{m}=\left[y_{m}^{(1)}, \ldots, y_{m}^{(n)}, \ldots, y_{m}^{\left(N_{m}\right)}\right]^{T} \in \mathbb{R}^{N_{m}}
$$

for which the derivation of the related probability distribution $p\left(\mathbf{y}_{m} \mid \mathbf{x},\left\{\mathbf{p}_{m}^{(k)}\right\}_{k=1}^{K_{m}}\right)$ is non-trivial and is dependent on the association of these $N_{m}$ measurements with the $K_{m}$ MPCs/VAs. This is a central part of this thesis and is discussed further in Chapter 5. We do however assume that each MPC/VA is never measured more than once. If this is a reasonable assumption or not, is highly dependent on the resolvability of the different individual MPCs/VAs as discussed in Section 2.5. In this work we assume that the bandwidth and hence the sampling rate is sufficiently high, so that full resolvability of the individual MPCs/VAs is possible.

We can further define the combined measurements over all $M$ anchors as

$$
\mathbf{z}=\left[\mathbf{y}_{1}^{T}, \ldots, \mathbf{y}_{M}^{T}\right]^{T} \in \mathbb{R}^{M N_{m}}
$$

for which we assume that a perfect association of each measurement $\mathbf{y}_{m}$ with the correct anchor $m$ is possible and that these measurements are independent. This is a reasonable assumption if, e.g., the transmission of the pulses $s(t)$ used to generate these measurements as discussed in Section 3.2, are well-separated in time-, frequency- and/or code-space as assumed here. This gives us

$$
p\left(\mathbf{z} \mid \mathbf{x},\left\{\left\{\mathbf{p}_{m}^{(k)}\right\}_{k=1}^{K_{m}}\right\}_{m=1}^{M}\right)=\prod_{m=1}^{M} p\left(\mathbf{y}_{m} \mid \mathbf{x},\left\{\mathbf{p}_{m}^{(k)}\right\}_{k=1}^{K_{m}}\right) .
$$




\subsubsection{Bi-Directional}

So far, as in the previous Section 3.3.1, we have considered only measurements and MPCs in one direction. For example, the downlink (DL) case where transmissions are made by the anchors and the agent performs the estimation based on the measured DL MPCs. In practice however, the corresponding measurements and MPCs in both directions could be considered jointly. Consider for example a scenario where the agent does not perform the estimation, but instead transmits a measurement report back to the measured anchor, after which the estimation is made on the anchor side. Alternatively, the anchors could measure the MPCs from UL transmissions by the agent, and thereafter return measurement reports back to the agent which will take care of the estimation. These both scenarios are depicted in Figure 3.3 and referred to as Anchor Triggered and Agent Triggered, respectively.

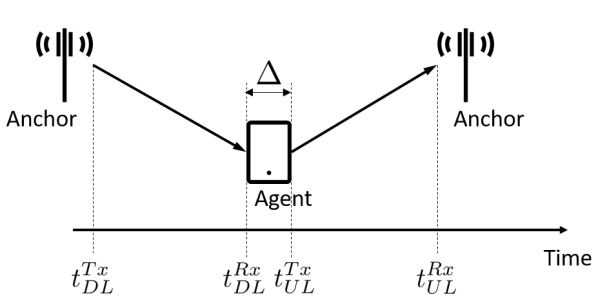

(a) Anchor Triggered

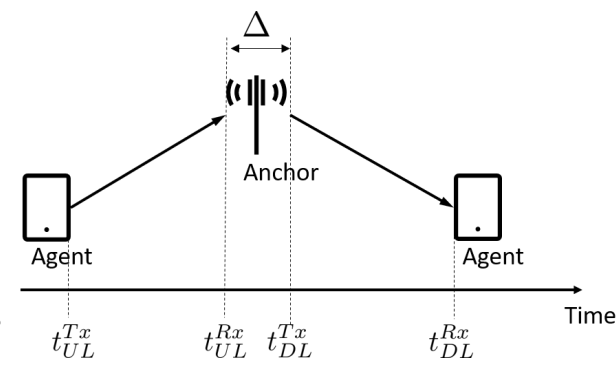

(b) Agent Triggered

Figure 3.3: Overview of different approaches, $\Delta$ is the (possibly known) processing delay at the measuring but non-estimating node.

Assuming that the non-estimating node is able to report all its measurements to the node performing the estimations, the resulting measurement vector can in both cases, given (3.17), be expressed as

$$
\mathbf{z}^{\mathrm{TOT}}=\left[\begin{array}{l}
\mathbf{z}^{\mathrm{DL}} \\
\mathbf{z}^{\mathrm{UL}}
\end{array}\right] \in \mathbb{R}^{M\left(N_{m}^{\mathrm{DL}}+N_{m}^{\mathrm{UL}}\right)}
$$

where the superscripts DL and UL are used for the respective direction. Assuming no cross-measurements in-between anchors and/or agents (i.e., that the measurements are performed on a per-anchor/per-agent basis only) and also assuming the noise to be mutually uncorrelated between the both directions, the resulting probability distribution is given by (3.18) and (3.19) as

$$
\begin{aligned}
p\left(\mathbf{z}^{\mathrm{TOT}} \mid \mathbf{x},\left\{\left\{\mathbf{p}_{m}^{(k)}\right\}_{k=1}^{K_{m}}\right\}_{m=1}^{M}\right) & =p\left(\mathbf{z}^{\mathrm{DL}} \mid \mathbf{x},\left\{\left\{\mathbf{p}_{m}^{(k)}\right\}_{k=1}^{K_{m}}\right\}_{m=1}^{M}\right) p\left(\mathbf{z}^{\mathrm{UL}} \mid \mathbf{x},\left\{\left\{\mathbf{p}_{m}^{(k)}\right\}_{k=1}^{K_{m}}\right\}_{m=1}^{M}\right) \\
& =\prod_{m=1}^{M} p\left(\mathbf{y}_{m}^{\mathrm{DL}} \mid \mathbf{x},\left\{\mathbf{p}_{m}^{(k)}\right\}_{k=1}^{K_{m}}\right) p\left(\mathbf{y}_{m}^{\mathrm{UL}} \mid \mathbf{x},\left\{\mathbf{p}_{m}^{(k)}\right\}_{k=1}^{K_{m}}\right) .
\end{aligned}
$$

It should be noted that in the general case, the UL and DL may be operating in different frequency bands, there may be different hardware involved in the 
agent and/or anchors for the respective directions, there can be the differences in-between different anchors etc. Therefore, the errors of (3.13) and hence the PDF of (3.14) and consequently the terms in the right-hand side of (3.20) will differ for the respective directions.

\subsection{Estimation of Agent Location}

Assuming that pseudo-range measurements are available for multiple MPCs/ VAs (as discussed in Section 3.3) we can perform a parameter estimation (as discussed in Section 3.1.1) of the unknown agent position $\mathbf{x}$ (assuming no prior knowledge thereof) given the measurements $\mathbf{z}$ of (3.17) which together with (3.18) gives

$$
\begin{aligned}
\hat{\mathbf{x}}_{\mathrm{ML}} & =\arg \max _{\mathbf{x}} p\left(\mathbf{z} \mid \mathbf{x},\left\{\left\{\mathbf{p}_{m}^{(k)}\right\}_{k=1}^{K_{m}}\right\}_{m=1}^{M}\right)=\arg \max _{\mathbf{x}} \ln \prod_{m=1}^{M} p\left(\mathbf{y}_{m} \mid \mathbf{x},\left\{\mathbf{p}_{m}^{(k)}\right\}_{k=1}^{K_{m}}\right) \\
& =\arg \max _{\mathbf{x}} \sum_{m=1}^{M} \ln p\left(\mathbf{y}_{m} \mid \mathbf{x},\left\{\mathbf{p}_{m}^{(k)}\right\}_{k=1}^{K_{m}}\right)=\arg \min _{\mathbf{x}} \sum_{m=1}^{M}-\ln p\left(\mathbf{y}_{m} \mid \mathbf{x},\left\{\mathbf{p}_{m}^{(k)}\right\}_{k=1}^{K_{m}}\right)
\end{aligned}
$$

which, as said before, in general is a complicated non-convex expression which allows an analytical solution only in some special cases.

For the state estimation as per Section 3.1.2, we simply follow the procedure described therein, i.e., by using the particle filter of Algorithm 1 where $\mathbf{x}_{t}$ is the unknown agent position and $\mathbf{z}_{t}$ contains the measurements at time $t=1, \ldots, T$. 



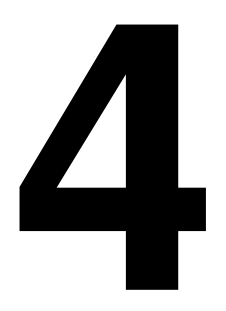

\section{TOA Estimation and Measurement Error Modelling}

This chapter deals with the choice of error models in the Time-of-Arrival (TOA) estimation process. It is based on the publication [4], but with some important extensions:

- We use the more refined radio channel model discussed previously in Chapter 2, as compared to [4] which used a more simplistic static model.

- We perform estimation of the agent location, and not just the distance between anchor and agent as was done in [4].

- The investigated set of error models are slightly different. Most importantly, we include both static error models based on actual accumulated error statistics as well as dynamic ones which are allowed to vary over time.

- We also include snapshot estimation, in addition to only the filtering in [4], since the latter may in some cases obfuscate the results and make the analysis more difficult.

In this chapter we do however limit the discussion to the uni-directional scenario discussed in Section 3.3.1, in contrast to [4] which also discussed the bidirectional case with a particular focus on Round-Trip Time (RTT) estimation. The principles from [4] with respect to RTT estimation are still valid but are omitted here since they are not central to this discussion.

This chapter is organized as follows: Section 4.1 presents how TOA measurements can be modelled using an estimation of the Multi-Path Component (MPC) representing the Direct Path (DP), also referred to as Line-of-Sight (LOS) component, and discusses the therewith associated errors. The following Section 4.2 introduces a number of measurement error models intended to improve the subsequent localization procedure. These models are then used in simulations for which the results are presented and discussed in Section 4.3, followed by a summary of the results in Section 4.4. Additional plots and results are provided in the appendix of Section 4.A. 


\subsection{DP/LOS Estimation and Measurements}

Instead of using range measurements for each available MPC as in Section 3.3, we can instead formulate the problem of estimating and using only the range associated with the DP MPC which represents the shortest Euclidean distance between transmitter and receiver. A common approach is to take the DP component as the first MPC with a received power within $3 \mathrm{~dB}$ of the strongest one. We let $\left\{\tau_{m}^{(k)}\right\}$ and $\left\{\alpha_{m}^{(k)}\right\}$ be the delays and amplitudes for the estimated MPC $k$ of anchor $m$. Assuming MPC $k_{\mathrm{DP}}$ to be the true DP we can then express its estimate in linear scale as

$$
\begin{aligned}
\hat{k}_{\mathrm{DP}} & =\underset{k \in\left\{1, \ldots, K_{m}\right\}}{\arg \min }\left\{\tau_{m}^{(k)}:\left|\alpha_{m}^{(k)}\right|^{2} \geq 10^{-3 / 10} P_{\max }\right\} \\
\text { where } P_{\max } & =\max \left\{\left|\alpha_{m}^{(k)}\right|^{2}: k \in\left\{1, \ldots, K_{m}\right\}\right\} .
\end{aligned}
$$

\section{Example 4.1}

In Example 3.1 we estimated the MPCs for an agent moving along the circular trajectory of Scenario A in Section 2.1. Continuing from this, Figure 4.1 shows the delay of the estimated DP by (4.1) (dash-dotted line). It also shows the delay in the ideal case without any AWGN/DM noise (dashed line) as well as that associated with the true Euclidean distance between anchor and agent (solid line).

Anchor 1
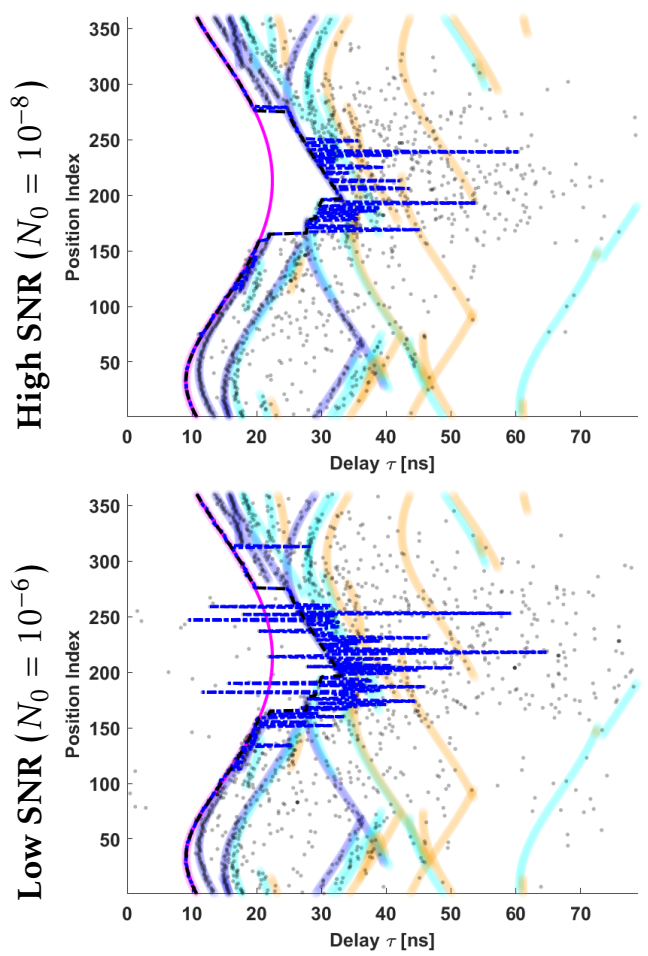

Anchor 2
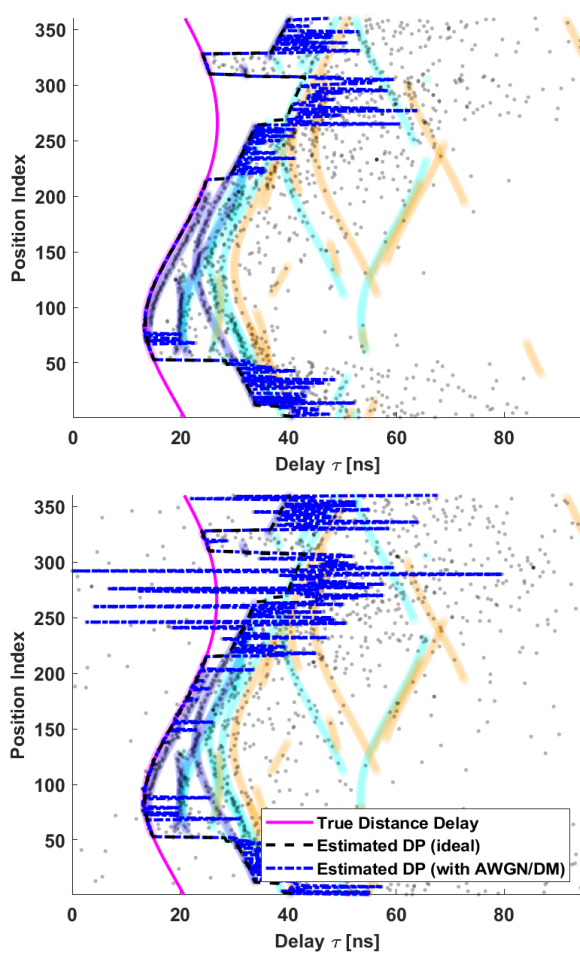

Figure 4.1: The estimated DP over the circular trajectory. 
As seen from Example 4.1, the estimated MPCs align well with the delay of the true distance during LOS conditions for the respective anchor in both the low and high SNR cases, but that we do get errors - especially during non-LOS conditions when the DM noise is significant. Even in the ideal case without any AWGN/DM noise, we still get errors due to the non-LOS bias. Further, in the low SNR case, we see the effect of misinterpreting noisy measurements as false MPCs.

We can formulate a pseudo-range measurement model in analogy with (3.13) for the DP between anchor $m$ located at the fixed position $\mathbf{p}_{m}$ and the agent located at $\mathbf{x}(t)$ as

$$
y_{m}^{\left(k_{\mathrm{DP}}\right)}(t)=\left\|\mathbf{x}(t)-\mathbf{p}_{m}\right\|_{2}+c f_{m}^{\left(k_{\mathrm{DP}}\right)}(t) \quad[m]
$$

where $c$ is the speed of light and $f_{m}^{\left(k_{\mathrm{DP}}\right)}(t)$ is the measurement error model. This error model should include not only the measurement/estimation error associated with the true DP which is MPC $k_{\mathrm{DP}}$ as such given by $e_{m}^{\left(k_{\mathrm{DP}}\right)}(t)$ in (3.13). It should also include the probability of picking the wrong MPC as the DP component, i.e., the probability of picking $k_{\mathrm{DP}} \neq k_{\mathrm{DP}}$. From Example 4.1 , we get an indication that assuming the DP to always be correctly identified by the receiving side, i.e., that $\hat{k}_{\mathrm{DP}}=k_{\mathrm{DP}}$, is an idealized and many times incorrect assumption. As discussed in e.g. $[10,21,35]$ as well as in [4], it may happen as in Example 4.1 that the DP is obscured and hence so weak that it is not even detected. Any measurement based on a non-DP MPC will cause an over-estimation of the range between agent and anchor. This is referred to as a Late Detection (LD) error. We also see that noise may be erroneously interpreted as MPCs (see also [23, 35]), potentially with a shorter delay than the DP and hence under-estimating the range between agent and anchor. This is referred to as a False Alarm (FA) error. These both types of errors are illustrated in Figure 4.2. How to capture all these types of errors in the error model $f_{m}^{\left(k_{\mathrm{DP}}\right)}(t)$ of $(4.2)$ is discussed in the following section.

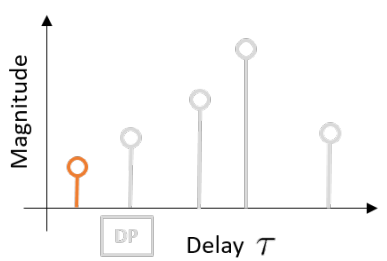

(a) False Alarm (FA)

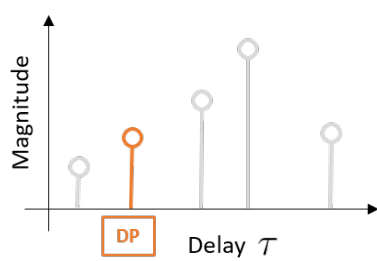

(b) Correct

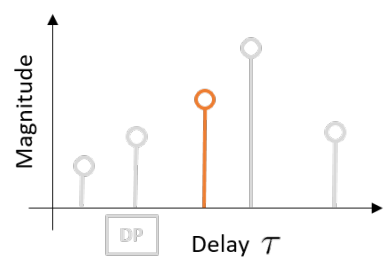

(c) Late Detection $(L D)$

Figure 4.2: Potential error cases where ' $D P$ ' indicates the true $D P$, and the coloured bar what the receiver believes to be the DP. The cases are: (a) False Alarm (FA) where noise is misinterpreted as the DP so that $\tau<\tau_{D P}$, (b) Correct behaviour where the DP is correctly interpreted so that $\tau=\tau_{D P}$ and (c) Late Detection (LD) where the DP is misinterpreted as noise so that $\tau>\tau_{D P}$. 


\subsection{Error Models}

We define the empirical/actual DP estimation error in terms of delay with respect to anchor $m$ located at the fixed position $\mathbf{p}_{m}$ and the agent located at $\mathbf{x}(t)$ as

$$
\operatorname{Err}_{m}^{\mathrm{DP}}(t)=\hat{\tau}_{m}^{\left(\hat{k}_{\mathrm{DP}}\right)}(t)-\frac{1}{c}\left\|\mathbf{x}(t)-\mathbf{p}_{m}\right\|_{2} \quad[s]
$$

where $\hat{\tau}_{m}^{\left(\hat{k}_{\mathrm{DP}}\right)}(t)$ is the estimated delay (as discussed in Section 3.2) with respect to the estimated DP MPC $\hat{k}_{\mathrm{DP}}$.

In this section we propose a number of models for the error $f_{m}^{\left(k_{\mathrm{DP}}\right)}(t)$ in $(4.2)$ which approximates the statistical properties of $\operatorname{Err}_{m}^{\mathrm{DP}}(t)$ in (4.3) as well as possible. In the design of these error models there is a trade-off between, on the one-hand, accuracy in the estimation and subsequent localization procedure and, on the other hand, the complexity in terms of, e.g., what needs to be reported in-between anchor and agent.

The proposed error models are divided in two subgroups: The first one is the static error models which does not change over time. The second set of models is the dynamic ones which may take on different values at different times.

\subsubsection{Static Error Models}

Starting with the static error models which does not change over time, a first naive assumption is to assume that the DP component is perfectly identified, i.e., that $\hat{k}_{\mathrm{DP}}=k_{\mathrm{DP}}$. As mentioned earlier, this may not be the optimal choice, but is intended to be used as a baseline assumption. Under this assumption, the only error is the one associated with the estimation of MPC $k_{\mathrm{DP}}$, i.e., $e_{m}^{\left(k_{\mathrm{DP}}\right)}(t) \sim$ $\mathcal{N}\left(0, \sigma_{m, k_{\mathrm{DP}}}^{2}\right)$ as in (3.13) where $\sigma_{m, k_{\mathrm{DP}}}$ is the therewith assumed standard deviation.

One question is how to determine a suitable value for the parameter $\sigma_{m, k_{\mathrm{DP}}}$. What we propose is to simply set this to the symbol duration of the transmitted UWB signal which is $T=1 \mathrm{~ns}$ as defined in Section 2.4. This may be a simplistic assumption since, as discussed in Example 2.4 of Section 2.5, the resolution of the estimated CIR will depend on the sampling rate. As we throughout this thesis assume $100 \times$ oversampling, it should be reasonable to assume that the receiver will (at least) not estimate any worse than one symbol duration wrong if the correct DP is chosen. Hence, in the numerical evaluations of Section 4.3 we use $\sigma_{m, k_{\mathrm{DP}}}=1 \mathrm{~ns}$ for all anchors $m$ and potential DPs $k_{\mathrm{DP}}$. Further, as seen in Example 4.1, the impact of erroneous DP identification is typically much larger than this value. Hence the impact of using this setting with respect to the overall errors should not be overly critical.

This gives us a set of error Models A1-A3:

Model A1 Perfect DP identification is assumed:

$$
f_{m}^{\left(k_{\mathrm{DP}}\right)}=e_{m}^{\left(k_{\mathrm{DP}}\right)} \sim \mathcal{N}\left(0, \sigma_{m, k_{\mathrm{DP}}}^{2}\right)
$$


Model A2 As Model A1, but increasing the variance with a factor 10:

$$
f_{m}^{\left(k_{\mathrm{DP}}\right)} \sim \mathcal{N}\left(0,10 \sigma_{m, k_{\mathrm{DP}}}^{2}\right)
$$

Model A3 As Model A1, but increasing the variance with a factor 40:

$$
f_{m}^{\left(k_{\mathrm{DP}}\right)} \sim \mathcal{N}\left(0,40 \sigma_{m, k_{\mathrm{DP}}}^{2}\right)
$$

Models A2 and A3 above have been included in order to provide a wider statistical support of the error distribution as compared to Model A1. This could (potentially) take care of incorrect DP identification errors, something which is investigated in the later numerical evaluations.

For a more refined model, we continue by using the errors (4.3) aggregated over the whole trajectory of Example 4.1. The corresponding error histograms are shown for each of the two anchors in Figure 4.3 together with the proposed error Models B and BS, which are based on these aggregated errors:

Model B An empirical distribution based on the average/expected value of the DP errors for all times $t$ :

$$
f_{m}^{\left(k_{\mathrm{DP}}\right)}=\mathbb{E}_{\tau}\left[\operatorname{Err}_{m}^{\mathrm{DP}}(t) \forall t\right],
$$

where the expectation is taken with respect to the delay $\tau$.

Model BS A model-based approximation of Model B where the false alarms (FAs) and late detections (LDs) discussed in the previous section and Figure 4.2, are modelled as uniform distributions over the range in which they occur (but with a margin of three standard deviations) and with a probability proportional to their occurrence. The correct estimation is modelled with the same Gaussian assumption as in Model A1:

$$
\begin{aligned}
f_{m}^{\left(k_{\mathrm{DP}}\right)} \sim(1 & \left.-P_{F A}-P_{L D}\right) \mathcal{N}\left(0, \sigma_{m, k_{\mathrm{DP}}}^{2}\right)+P_{F A} \mathcal{U}\left(\min _{\forall t}\left(\operatorname{Err}_{m}^{\mathrm{DP}}(t)\right)-3 \sigma_{m, k_{\mathrm{DP}}}, 0\right) \\
& +P_{L D} \mathcal{U}\left(0, \max _{\forall t}\left(\operatorname{Err}_{m}^{\mathrm{DP}}(t)\right)+3 \sigma_{m, k_{\mathrm{DP}}}\right) .
\end{aligned}
$$

In (4.8), the probability of FAs $P_{F A}$ is determined as

$$
P_{F A}=\frac{1}{N_{t}} \sum_{\forall t} \mathbf{1}_{\mathrm{Err}_{m}^{\mathrm{DP}}(t)<-3 \sigma_{m, k_{\mathrm{DP}}}},
$$

where $\mathbf{1}_{X}$ is the indicator function for the event $X$, i.e.,

$$
\mathbf{1}_{X}= \begin{cases}1 & \text { if } X \text { is true } \\ 0 & \text { otherwise }\end{cases}
$$

and $N_{t}=\sum_{\forall t} 1$ is the total number of measurements in trajectory. Similarly, the probability of late detections $P_{L D}$ is determined as

$$
P_{L D}=\frac{1}{N_{t}} \sum_{\forall t} \mathbf{1}_{\mathrm{Err}_{m}^{\mathrm{DP}}(t)>3 \sigma_{m, k_{\mathrm{DP}}}} .
$$




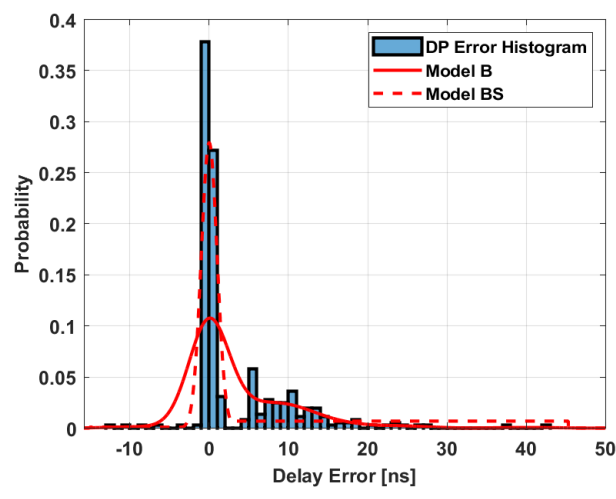

(a) Anchor 1

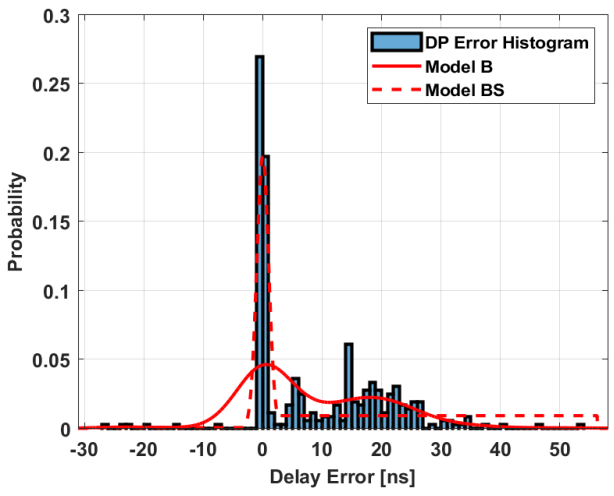

(b) Anchor 2

Figure 4.3: DP error histogram and error models $B / B S$.

An alternative to use the DP estimation error of (4.3) is to instead use the estimation error for each MPC $k=1, \ldots, K$ :

$$
\operatorname{Err}_{m}^{(k)}(t)=\hat{\tau}_{m}^{(k)}(t)-\frac{1}{c}\left\|\mathbf{x}(t)-\mathbf{p}_{m}\right\|_{2} \quad[s]
$$

Note that (4.11) do not take into account the DP estimation of (4.1) as done in Models B and BS, which is a simplification.

Aggregating the errors of (4.11), the corresponding error histograms are shown for each of the two anchors in Figure 4.4. Based on these, we propose the two error models $\mathrm{C}$ and CS:

Model C As Model B, but based on the expected value of the errors for all MPCs $k$ and times $t$ as per (4.11):

$$
f_{m}^{\left(k_{\mathrm{DP}}\right)}=\mathbb{E}_{\tau}\left[\operatorname{Err}_{m}^{(k)}(t) \forall k, t\right]
$$

Model CS A model-based approximation of Model C, defined in analogy with Model BS. 


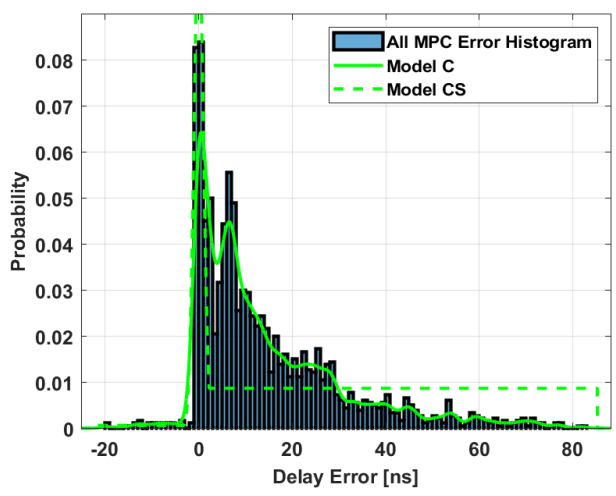

(a) Anchor 1

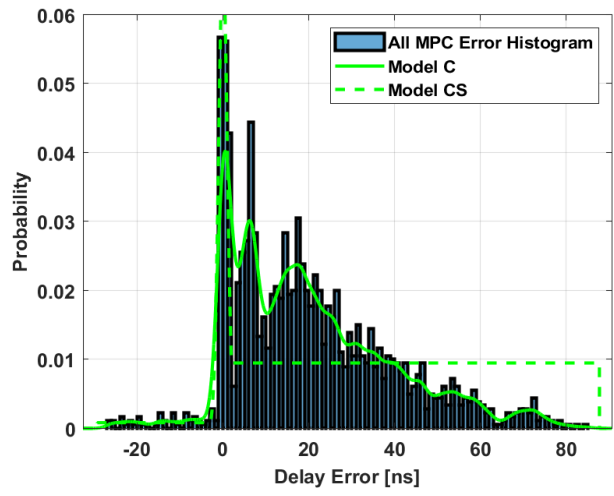

(b) Anchor 2

Figure 4.4: Error histogram for all MPC errors and error models C/CS.

All static error models derived in this section are gathered in Figure 4.5 below.

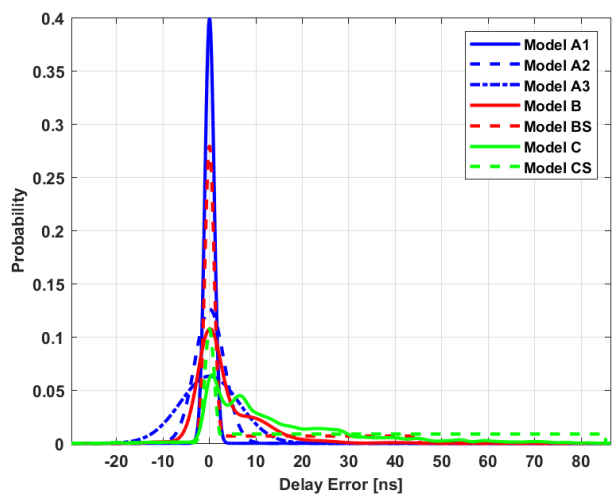

(a) Anchor 1

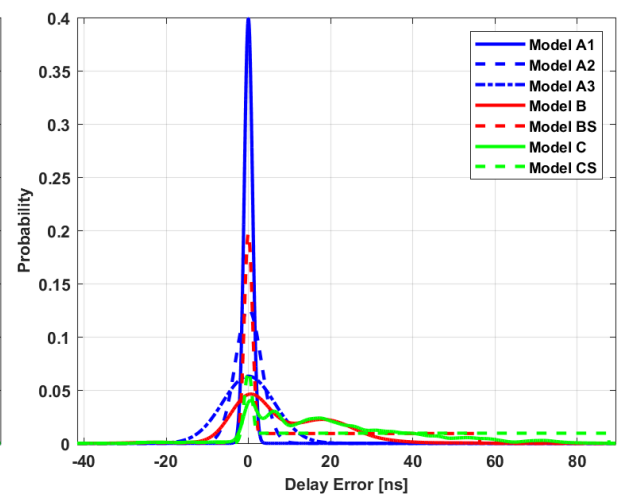

(b) Anchor 2

Figure 4.5: All static error models.

\subsubsection{Dynamic Error Models}

In this section dynamic error models are considered, which can change over time. We here attempt to at each time $t$ utilize the estimated MPCs as described in Section 3.2. This is similar to what we did for the static error Model D, but here using the set of errors $\left\{\operatorname{Err}_{m}^{(k)}(t)\right\}$ of (4.11) for all estimated MPCs $k=1, \ldots, K$ at each time $t$ rather than aggregated over time. Again, this does not take into account the actual DP estimation of (4.1). We propose the dynamic error Models $\mathrm{D}, \mathrm{DS}$ and $\mathrm{E}$ :

Model D A Gaussian mixture model (GMM) constructed based on the error of all 
the $K$ estimated MPCs at time $t$ given by (4.11):

$$
f_{m}^{\left(k_{\mathrm{DP}}\right)}(t) \sim \sum_{k=1}^{K} w_{m}^{(k)}(t) \mathcal{N}\left(\operatorname{Err}_{m}^{(k)}(t), \sigma_{m, k}^{2}\right)
$$

where the weight of each component is proportional to the estimated received power of the respective MPC:

$$
w_{m}^{(k)}(t)=\frac{1}{\sum_{k^{\prime}=1}^{K}\left|\hat{\alpha}_{m}^{\left(k^{\prime}\right)}(t)\right|^{2}}\left|\hat{\alpha}_{m}^{(k)}(t)\right|^{2} .
$$

Model DS A model-based approximation of Model D, defined in analogy with Model BS and CS.

Model E This is the ideal model having perfect knowledge about FA, LD and nonLOS errors. To cater for the actual MPC estimation errors, we still model it using the same Gaussian distribution as in Model A1, but now always centred around the true value of the DP error, i.e.,

$$
f_{m}^{\left(k_{\mathrm{DP}}\right)}(t) \sim \mathcal{N}\left(\operatorname{Err}_{m}^{\left(k_{\mathrm{DP}}\right)}(t), \sigma_{m, k_{\mathrm{DP}}}^{2}\right)
$$

For illustrative purposes, we show in Figure 4.6 these dynamic error Models D, DS and $E$ at a few different selected positions $\{1,136,226\}$ along the circular trajectory of Scenario A. The placement of these positions (which are shown later in Figure 4.8 of Section 4.3) have LOS between the agent and one, both and no anchor, respectively. In Figure 4.6 we also illustrate the DP estimation error (4.3) as well the error for all MPCs (4.11) at that very position. From this figure we see that Model D gives probability mass around each of the estimated MPCs where the largest value in some, but not all cases, coincides with the DP error. The reason for this discrepancy, as seen at Position 1 for Anchor 2 and Position 226 for Anchor 1, is that the total probability mass of (4.13) for two adjacent MPCs will add up to a larger value. Another choice of weights rather than just the received power of the respective MPC could be beneficial such as, e.g., always giving the estimated DP the largest value or - even better - factoring in the probability of correct estimation according to (4.1). These refinements are however left for future studies. The approximate Model DS gives a probability peak around 0 , if that is spanned by the estimated MPCs, but otherwise modelled by the uniform distributions as per $(4.8)-(4.10)$ (but with $k$ rather than $\left.k_{\mathrm{DP}}\right)$. The ideal Model $\mathrm{E}$ is always (per construction) centred around the true DP error (4.3). 

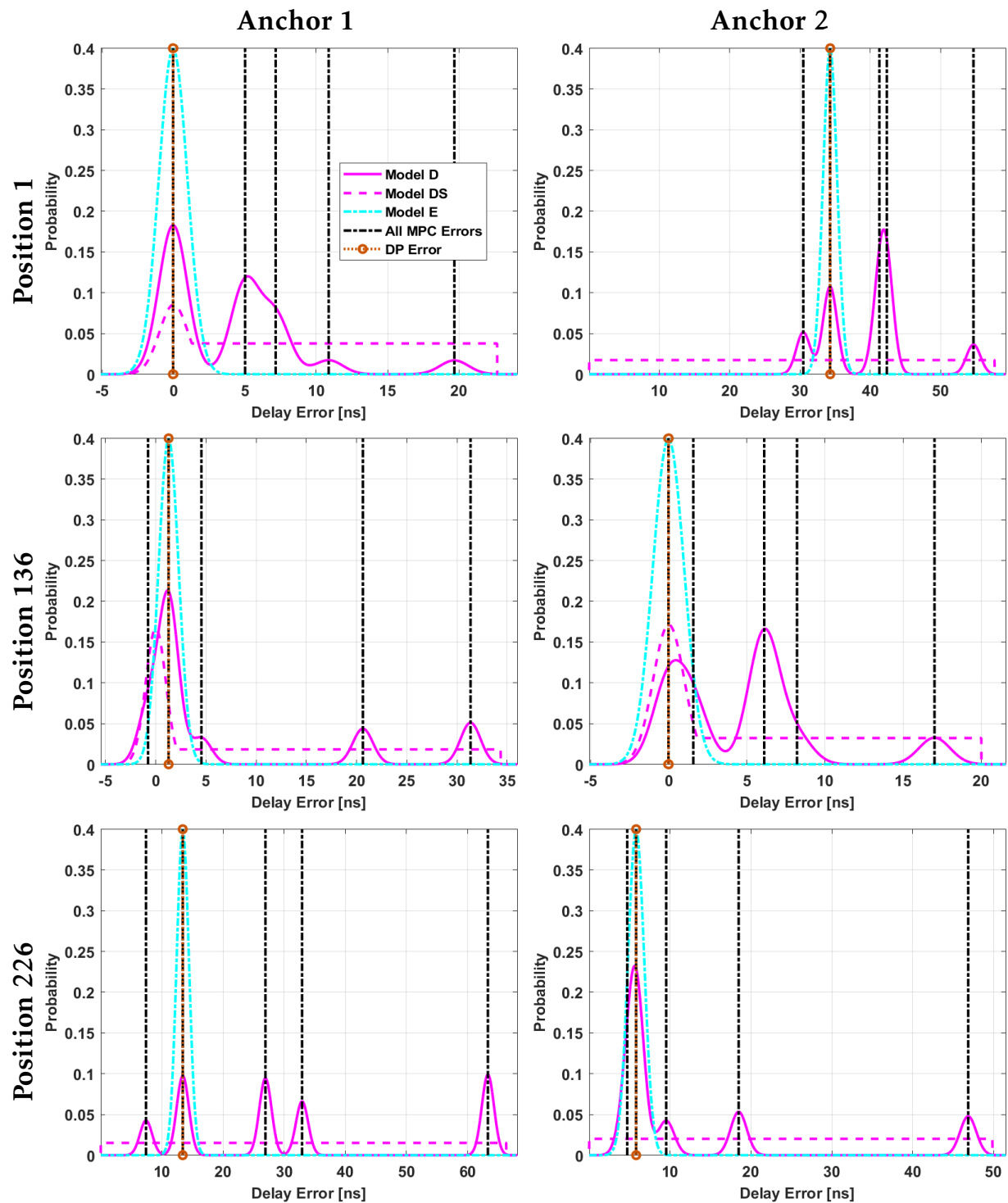

Figure 4.6: The probability distributions for the dynamic error models $D$, $D S$ and $E$ for both anchors at the different positions 1, 136 and 226. The DP estimation error (4.3) as well the error for all MPCs (4.11) at the respective position is also shown.

The full probability traces, i.e., the corresponding probabilities over the whole trajectory for these error models, is shown in Figure 4.7. Comparing with Figure 4.8 we see a clear correlation between the non-LOS conditions for the respective anchor and these traces. Thus, Model E represents the perfect knowledge whereas the other models are approximations thereof. 

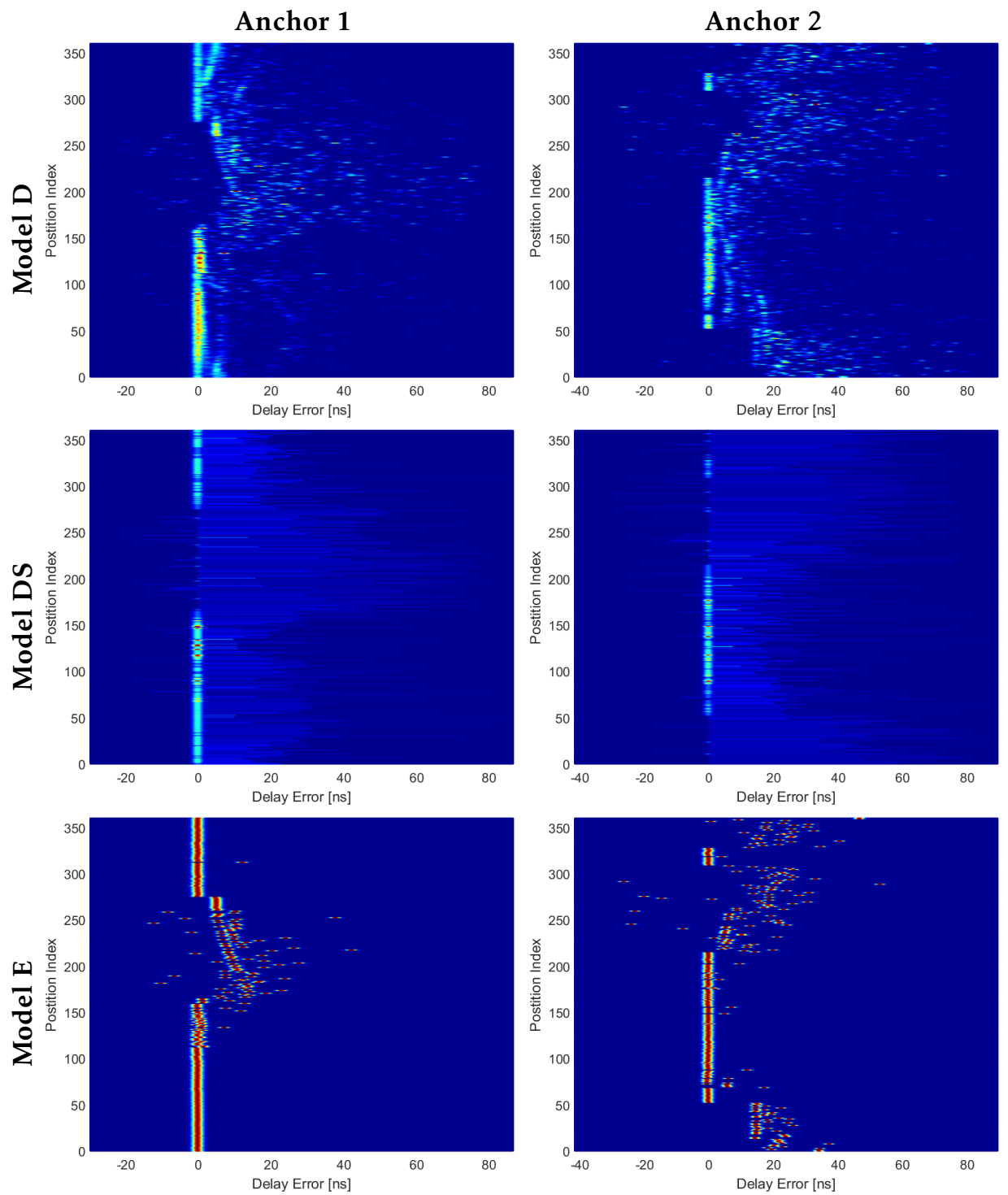

Figure 4.7: The probability distributions for the dynamic error Models $D, D S$ and $E$ for both anchors plotted over the whole agent trajectory of Scenario A.

\subsection{Numerical Results}

The evaluations are performed for each error model proposed in Section 4.2. This is done over the circular trajectory of Scenario A in Section 2.1, and hence essentially continues the series of examples presented earlier in this thesis. The performance metric used in the evaluations is the localization error $\|\mathbf{x}(t)-\hat{\mathbf{x}}(t)\|_{2}$ for each time-step $t$, where $\mathbf{x}(t)$ is the true position of the agent and $\hat{\mathbf{x}}(t)$ is the estimated position (discussed in Section 3.4). We focus solely on the low SNR 
case (defined in Table 2.1 of Section 2.1) since this presents the largest amount of errors and error types, and hence is deemed the most general.

Figure 4.8 shows, for each anchor, the number of visible virtual anchors (VAs) at each point of the trajectory as well as whether the considered point is LOS or not. In also indicates three positions $\{1,136,226\}$ which has LOS between the agent and one, both and no anchor, respectively. The measurement probability plots for these points are presented in the appendix of Section 4.A.

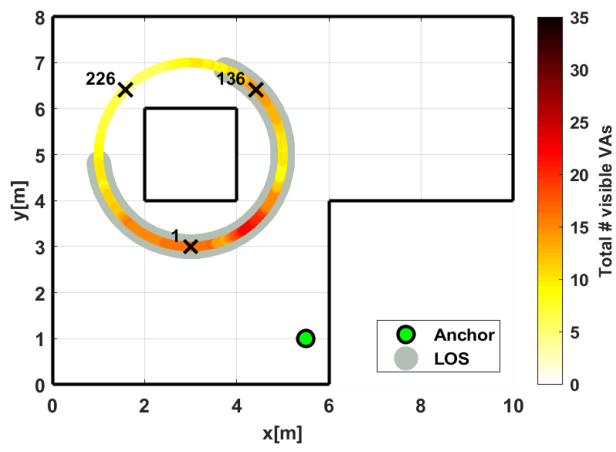

(a) Anchor 1

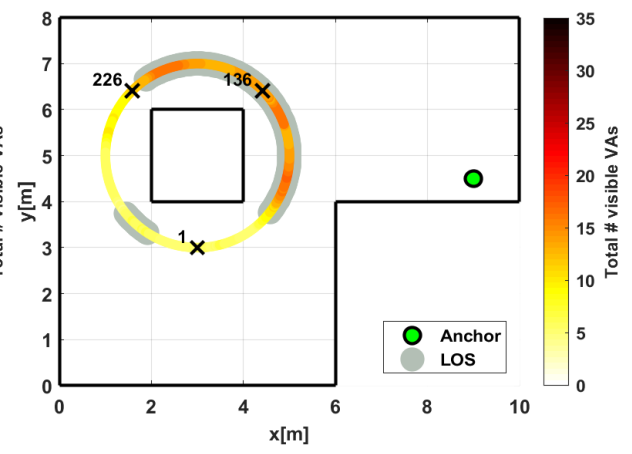

(b) Anchor 2

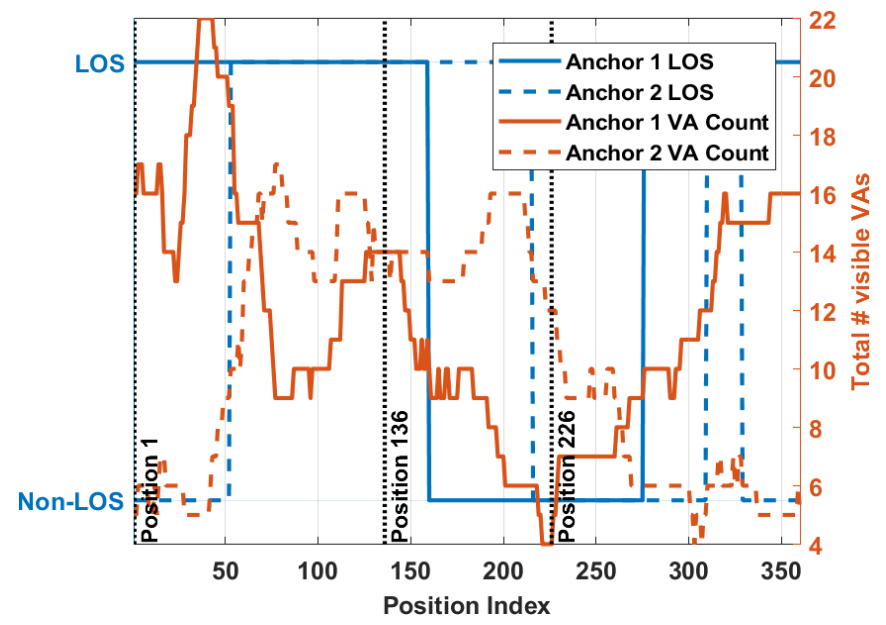

(c) Time plot

Figure 4.8: The total number of visible VAs as well as the LOS-regions for the respective anchors over the trajectory of Scenario A. The three different positions $\{1,136,226\}$ are indicated along the trajectory.

\subsubsection{Snapshot Estimation Results}

We start by performing ML estimation in a snapshot manner, i.e., considering only the measurements from each trajectory point as discussed in Section 3.1.1. All evaluations are performed using a grid-search over a $5 \times 5 \mathrm{~cm}$ grid covering the room with about $10 \mathrm{~m}$ margin and assuming no prior information of the room 
layout (denoted 'Full Grid'). We also consider the case when assuming the room layout to be known and hence exclude points not being inside the room (denoted 'Constrained to Room'). The resulting plots of the localization error for each of the error models presented in Section 4.2 along the trajectory is shown in Figure 4.9. Even though the noise makes it difficult to catch all the details, we still note that:

- The localization accuracy is best when having LOS to both anchors and significantly worse otherwise regardless of error model. This is as expected since we try to estimate two parameters (i.e., the $x$ and $y$ positions of the agent) using two or fewer measurements. This gives rise to ambiguous and multi-modal solutions including non-LOS bias which is not solvable by any commonly known standard method. It is therefore required to provide means to make this solvable. This topic is also further addressed in the subsequent chapters.

- The localization accuracy in the constrained case is significantly better for all error models. This is due to a number of reasons:

- The entire set of estimation grid-points is smaller, and hence any estimated position will be closer to the true one.

- Erroneous maxima in the resulting multi-modal probability distributions often ends up outside the room and hence are pruned away. This happens even for the ideal model E.

- The dynamic error models D and DS perform not very well, and even worse than the static error models, during the period with LOS to both anchors. This can be explained by the results of Figure 4.6 where we see that, e.g., for anchor 2 at positions 1 and 136, some of the MPCs defining the Gaussian mixture are quite close. This causes the resulting probability distribution to end up with a higher probability there instead of around the true DP error. Therefore, the static ML estimation will pick this point instead of the correct one.

The corresponding CDFs are shown in Figure 4.10. In addition to the above made observations, these plots show that:

- Having no error model, which essentially means using Model A1, can give good results in some positions when there are no FA nor LD errors. In general however, it performs quite poorly, highlighting the need for better error models.

- The width of the distribution matters, especially in the full-grid estimation, since, e.g., models A2 and A3 perform better than Model A1. For the constrained models this is not seen to the same extent.

- It appears as if the simplified models BS, CS and DS are worse than the full non-simplified ones B, C and D. Again, this is due to the static ML estimation, which simply picks the maximum point in these distributions, which in turn is nothing but the Gaussian peak of Model A1. Still, they do perform slightly better than Model A1. 
- The localization accuracy using dynamic error models are in general, despite the above-mentioned limitations, better than when using static ones. Still, the accuracy is no better than approximately $10^{0.25} \approx 1.8$ meters for the $80^{\text {th }}$ percentile in the constrained case and far from the ideal Model E.
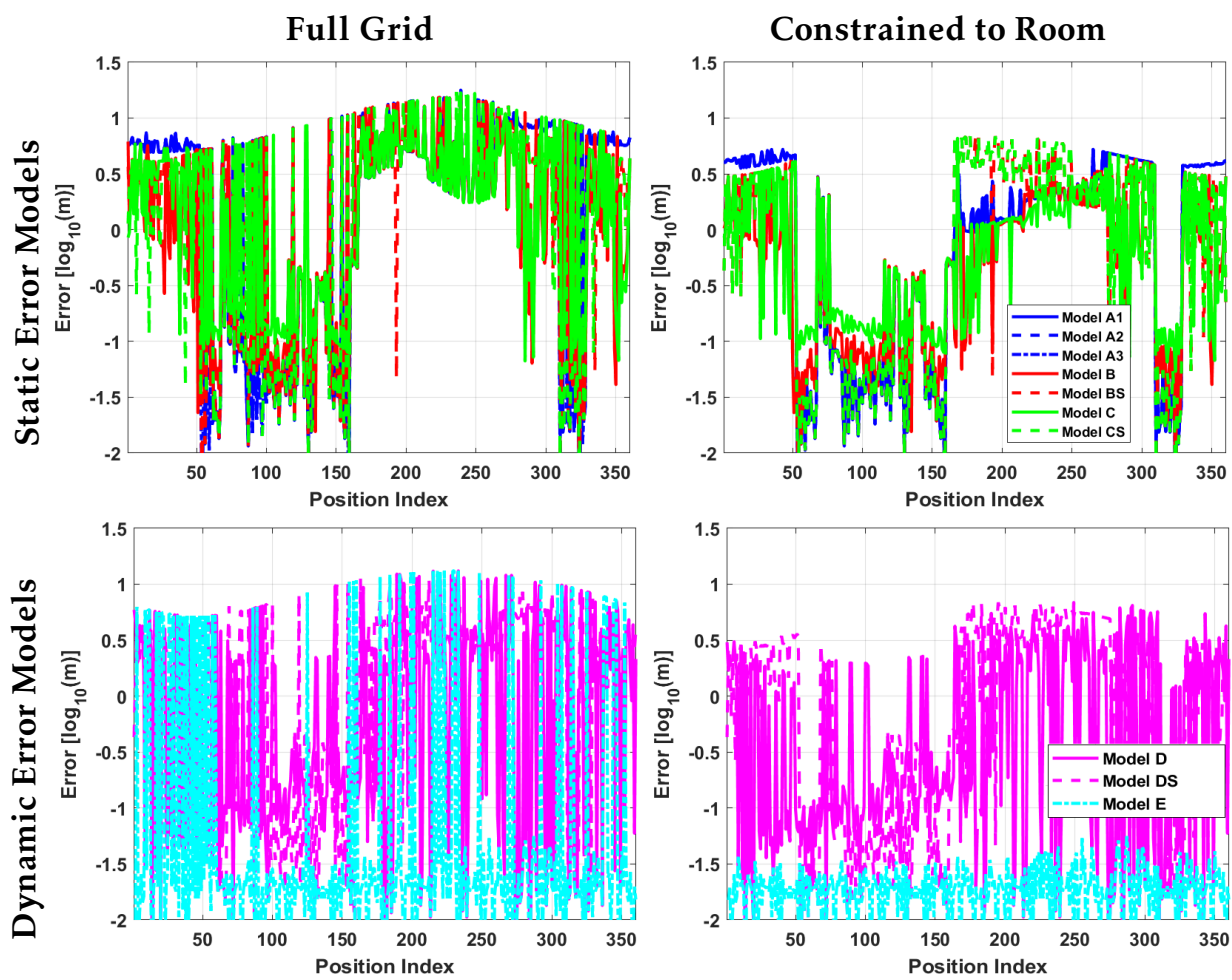

Figure 4.9: The localization error over the trajectory for all error models.

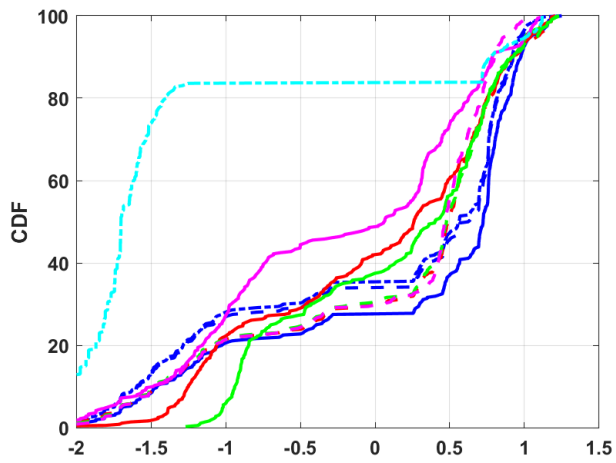

(a) Estimation over Full Grid

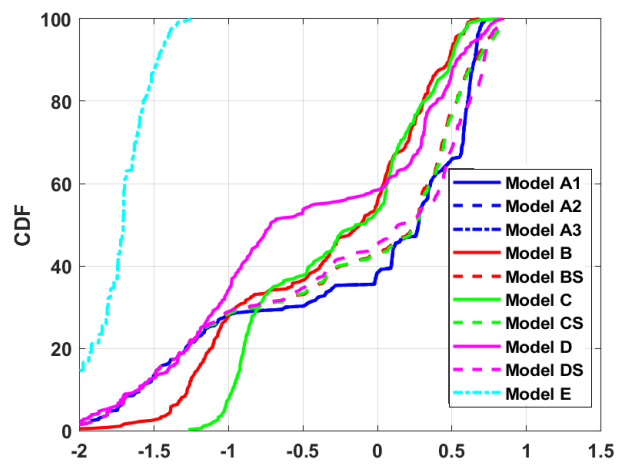

(b) Estimation Constrained to Room

Figure 4.10: CDFs of the localization error over the trajectory. 


\subsubsection{Filtering Results}

We now move on to filtering using a Sequential Importance Resampling (SIR) Particle Filter (PF) $([16,42])$ as described in Section 3.1.2. The number of particles is $N=100$ and the resampling threshold is set to $N_{\text {res }}=N$, i.e., resampling is performed at every time step making it a so-called Bootstrap PF. Furthermore, we do not assume any prior knowledge of the room layout, i.e., what was denoted 'Constrained to Room' in Section 4.3.1, which could be used to discard particles of the PF deemed invalid. The measurement model is the same as the one used so far in this chapter, i.e., as per (4.2), for the respective measurement error model. To model the anticipated motion of the agent, we use a simple two-dimensional (nearly) constant velocity (CV) motion model (see e.g. [16]):

$$
\left(\begin{array}{c}
x(t+T) \\
y(t+T) \\
v_{x}(t+T) \\
v_{y}(t+T)
\end{array}\right)=\left[\begin{array}{llll}
1 & 0 & T & 0 \\
0 & 1 & 0 & T \\
0 & 0 & 1 & 0 \\
0 & 0 & 0 & 1
\end{array}\right]\left(\begin{array}{c}
x(t) \\
y(t) \\
v_{x}(t) \\
v_{y}(t)
\end{array}\right)+\left[\begin{array}{cc}
T^{2} / 2 & 0 \\
0 & T^{2} / 2 \\
T & 0 \\
0 & T
\end{array}\right] a(t)
$$

where $a(t) \sim \mathcal{N}\left(0, \sigma_{a}^{2}\right)$ is the white process noise. We use a sample time of $T=1$ $\mathrm{s}$, a process noise standard deviation of $\sigma_{a}=0.1 \mathrm{~m} / \mathrm{s}^{2}$ and set the initial state to the true starting position of the agent but with some uncertainty:

$$
\left(\begin{array}{c}
x(0) \\
y(0) \\
v_{x}(0) \\
v_{y}(0)
\end{array}\right) \sim \mathcal{N}\left(\left[\begin{array}{l}
3 \\
3 \\
0 \\
0
\end{array}\right],\left[\begin{array}{llll}
2 & 0 & 0 & 0 \\
0 & 2 & 0 & 0 \\
0 & 0 & 1 & 0 \\
0 & 0 & 0 & 1
\end{array}\right]\right)
$$

The resulting plots of the estimated trajectory is plotted together with the room layout and the true trajectory in Figure 4.11 and the resulting time plot and CDF over the localization error is shown in Figure 4.12. We see that:

- Always assuming the DP to be correctly estimated as in Model A1 performs just as bad as before. Models A2 and A3 however improve the performance. This is because a wider distribution gives weight to a larger set of particles in the PF and hence increases the probability to capture the 'correct' error hypothesis.

- As in the earlier snapshot estimation, for all error models the localization accuracy is much better during the period when we have Los between the agent and both anchors as compared to when having only LOS to one or none of the anchors due to the non-LOS bias (which is implicitly removed by the ideal/genie Model E).

- The dynamic error models perform significantly better than the static ones almost everywhere, and vastly better than in the snapshot estimation of the previous section. The key reason for this is that here we do not perform the previously mentioned ML estimation, which causes problems when picking 'the wrong peak' as discussed on page 48 for Models D and DS. Instead, 
the different peaks of the distribution provide the support for which some particles are generated. The best accuracy we get for the $80^{\text {th }}$ percentile using model $\mathrm{D}$ is around $10^{-0.5} \approx 0.3$ meters and hence closer to the ideal model $\mathrm{E}$ which is $10^{-0.9} \approx 0.1$ meters.

- Even for the static error models, basing the error model on actual statistics is beneficial since, e.g., the performance of models B and BS are significantly better than for models A1-A3. It appears however as if Models C and CS do not perform that well. One possible explanation for this is that these models do not really take into consideration the DP estimation of (4.1) as such, but rather just includes the error wrt. all estimated MPCs.

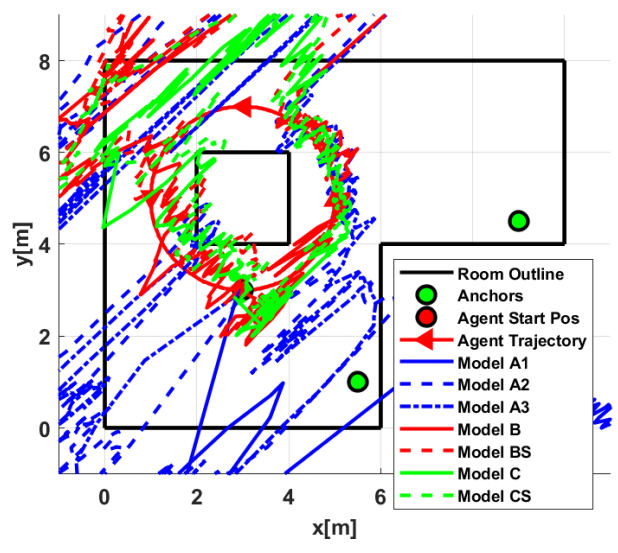

(a) Static Error Models

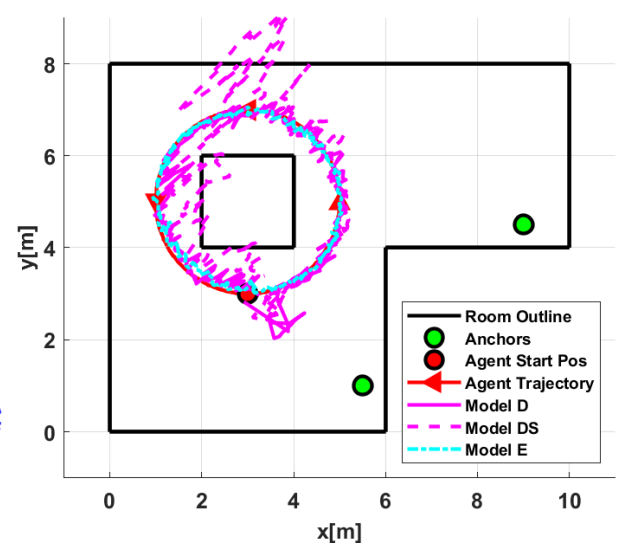

(b) Dynamic Error Models

Figure 4.11: The estimated trajectory using filtering.

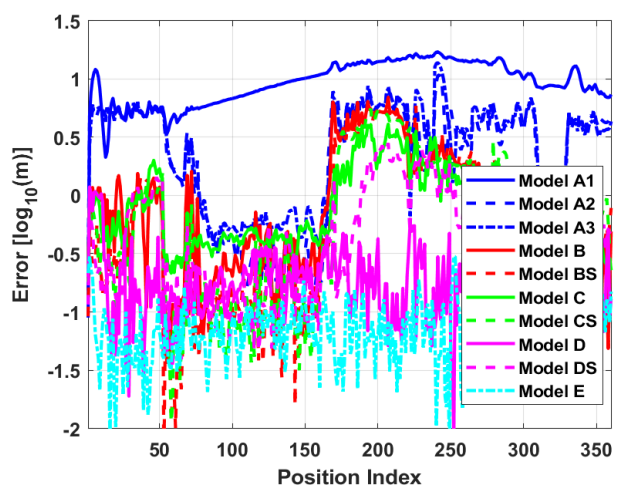

(a) Timeplot

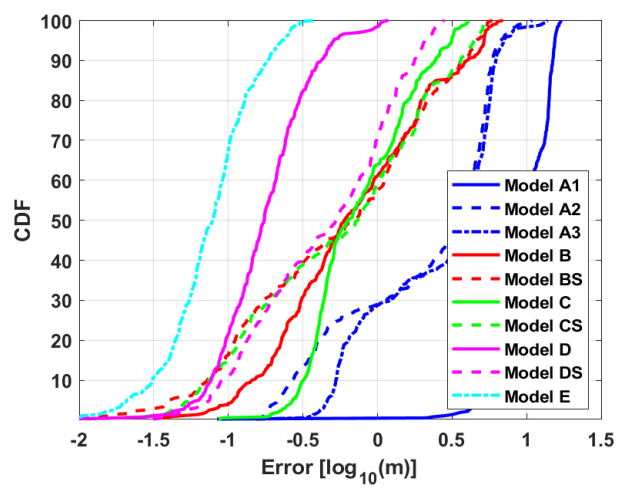

(b) $C D F$

Figure 4.12: The localization error over the trajectory using filtering. 


\subsection{Summary}

In this chapter we have analysed TOA measurements with respect to DP/LOS estimation. In particular, the impact different choices of measurement error models may have on the localization accuracy has been studied. We proposed a number of such error models of varying degrees of complexity, which have all been evaluated in simulations. From these results we have noted that:

- Estimation during non-LOS conditions is problematic, regardless of error model. By always assuming correct DP identification, as done in models $\mathrm{A} 1-\mathrm{A} 3$, is therefore not a good assumption even though this is some cases give reasonable estimates.

- Static error models based on aggregated/average DP error statistics, as Models B and BS, perform significantly better. Dynamic error models which considers the current measurements (instead of the aggregated / average ones) as models D and DS are even better in the filtering case. During snapshot ML estimation however, these models also get stuck in the local probability maxima.

- The most important aspect of any error model is that its resulting probability distribution covers the correct regions. For static ML estimation its highest peak should be as close as possible to the truth for the best estimation, but in the PF filtering case it is necessary only that enough probability mass is provided so that the corresponding particles survive.

- A final observation is that prior information of the environment, i.e., a map, is beneficial since it allows to prune away erroneous maxima and limit the search grid. Also, applying filtering (in this case a PF), as expected greatly improves the location accuracy through the use of multiple measurements and motion models.

To improve the localization accuracy, which even with filtering is not as good as we may desire and far from the ideal case, we see some ways forward including:

Non-LOS detection and/or mitigation One could try to determine if the measurement with respect to a given anchor is subject to non-LOS conditions and, if so, attempt to estimate/track the Los bias to compensate for it in the estimation procedure. Here, this was done implicitly by e.g. the ideal Model E, but more practical approaches are discussed in e.g. [21, 33, 50].

Utilizing Multipath Information In this chapter, we estimate the DP/LOS component and model the associated error (at least for the non-trivial models) by using the estimated MPCs. We could instead consider using all MPCs for positioning purposes as already mentioned in the last few paragraphs of Section 1.1, and hence utilize the 'full measurements' of Section 3.3.

In the remaining parts of this thesis, we focus on the latter of these two. This do however pose the problem of how to correctly associate a given measurement with the correct MPC/VA, which is the topic addressed by the following Chapter 5 . Thereafter the theoretical performance of this approach is assessed in Chapter 6. 


\section{Appendix}

\section{A Additional Figures}

This appendix contains additional probability plots for the respective error models of this chapter at the three selected positions 1, 136 and 226 over the circular trajectory of Scenario A as indicated in Figure 2.11 of Section 4.3.

These probability plots are presented both when evaluated over a rather large $5 \times 5 \mathrm{~cm}$ search grid assuming no prior information of the room layout, as well as when indeed assuming the room layout to be known and hence excluding points not being inside the room.

In all cases, the evaluations are done for the low SNR case, i.e., with $N_{0}=10^{-6}$. 

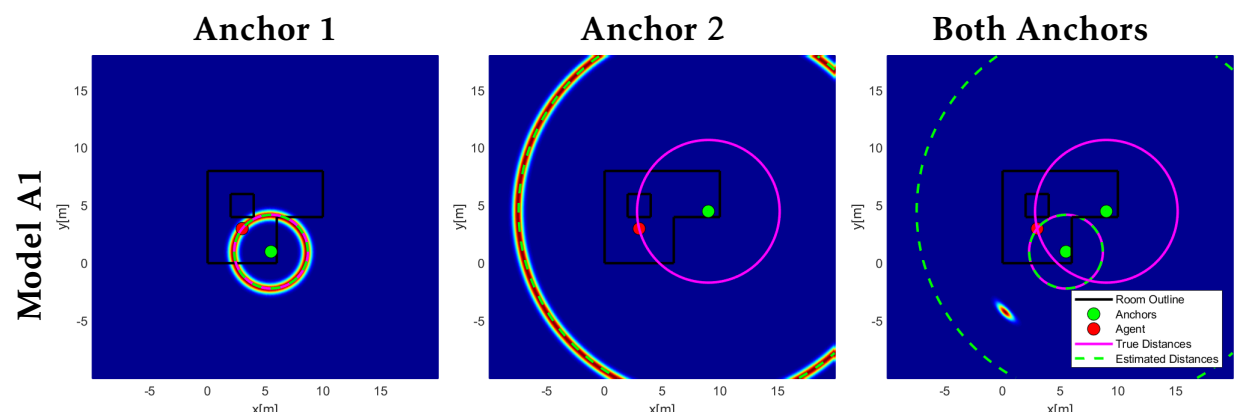

$x[\mathrm{~m}]$
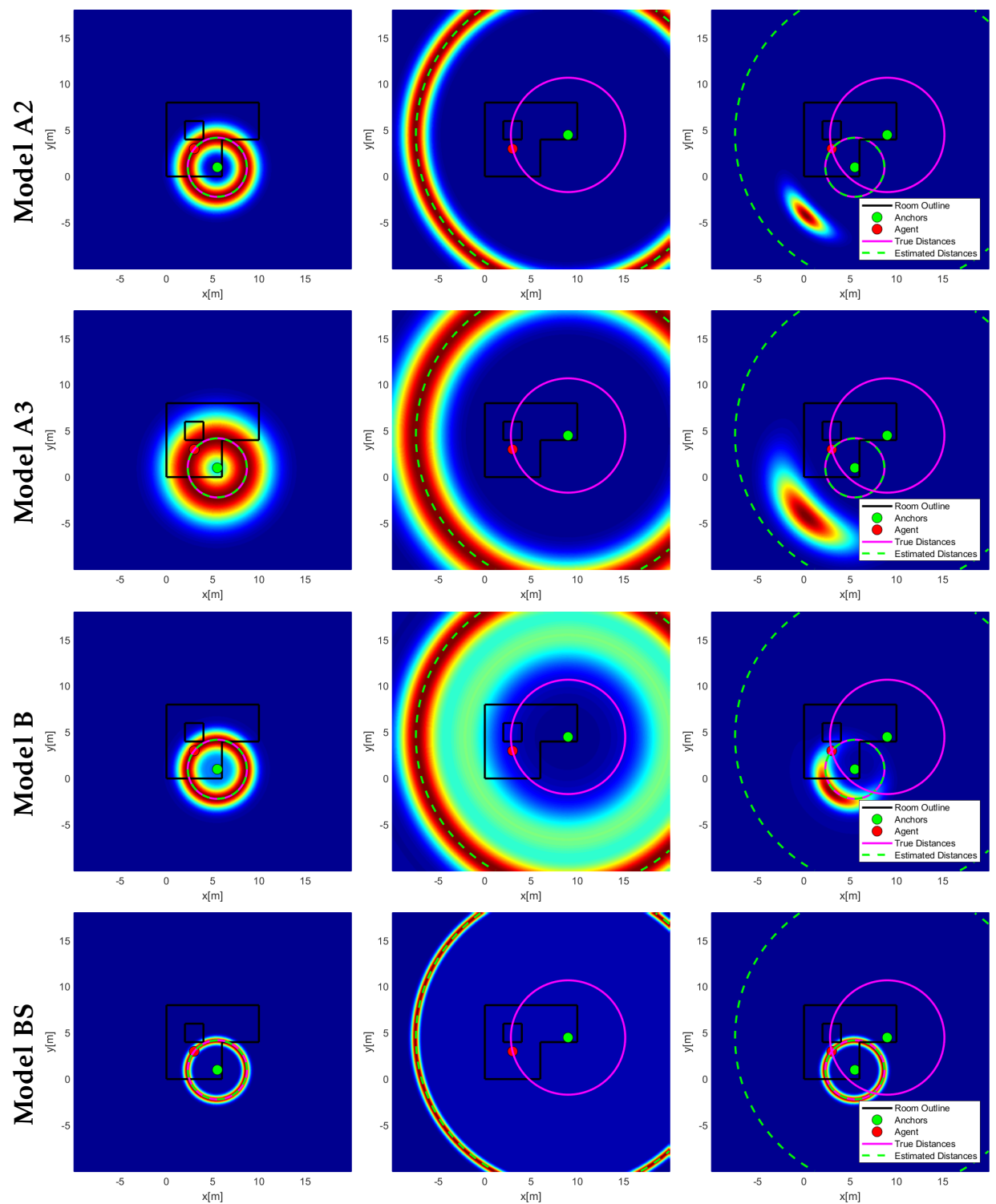

Figure 4.13: The probability distributions for the static error models $A 1, A 2$, $A 3, B$ and $B S$ at position 1 for low SNR, i.e., $N_{0}=10^{-6}$. 

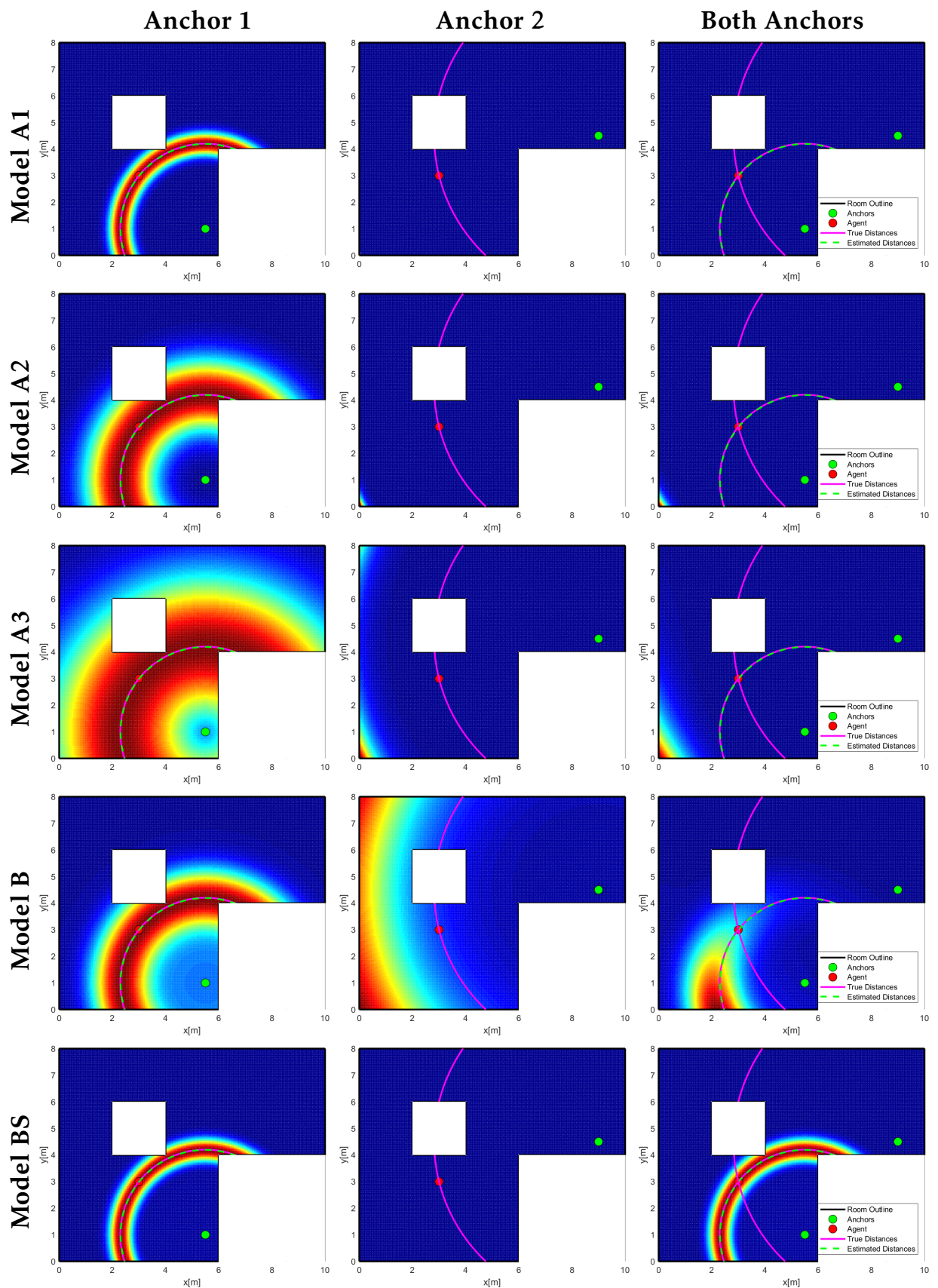

Figure 4.14: Same as Figure 4.13, but constrained to points within the room. 


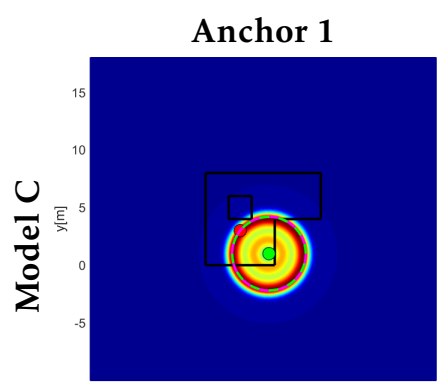

$x[\mathrm{~m}]$
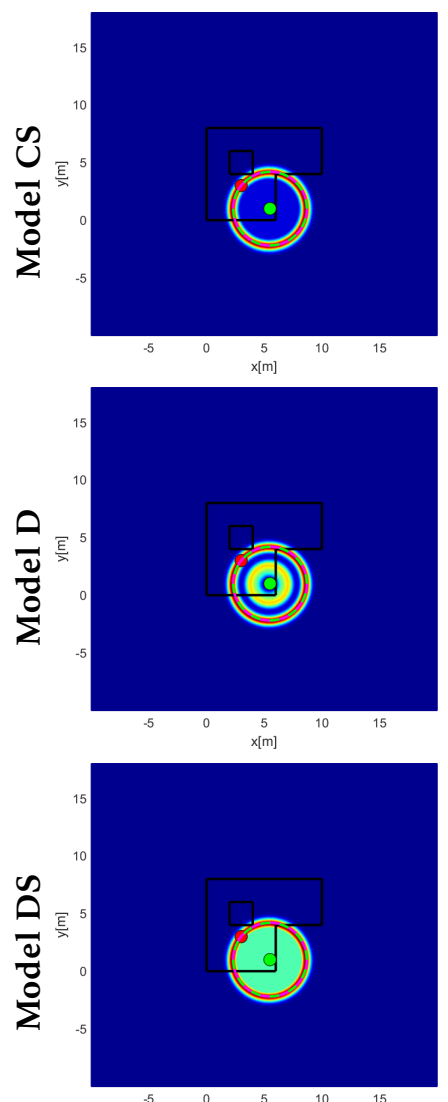

$\mathrm{x}[\mathrm{m}]$

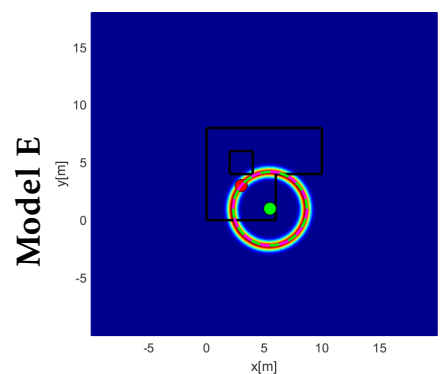

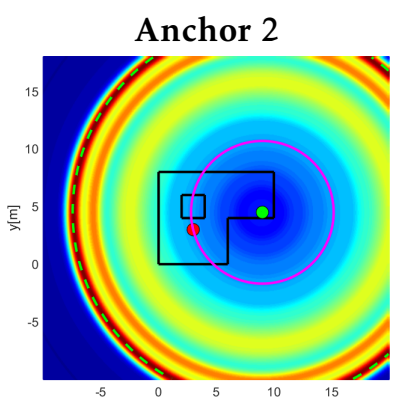

$x[\mathrm{~m}]$
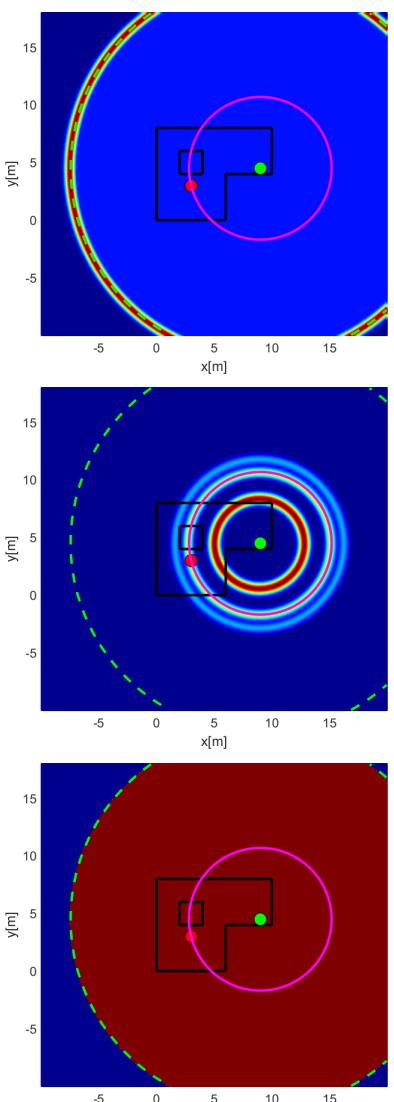

$x[\mathrm{~m}]$

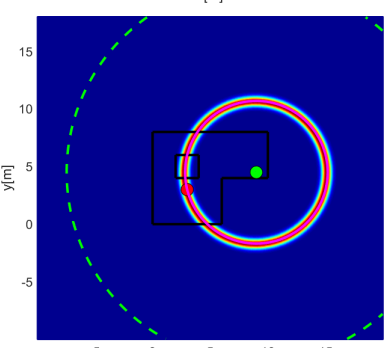

5
$x[\mathrm{~m}]$

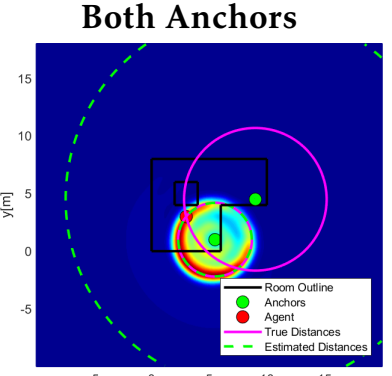

$\mathrm{x}[\mathrm{m}]$

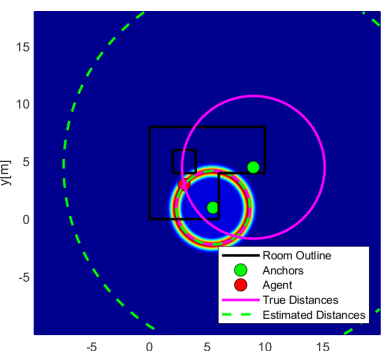

$\mathrm{x}[\mathrm{m}]$
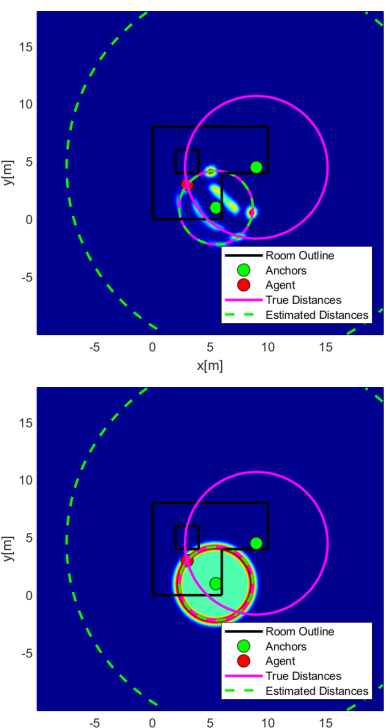

$\mathrm{x}[\mathrm{m}]$

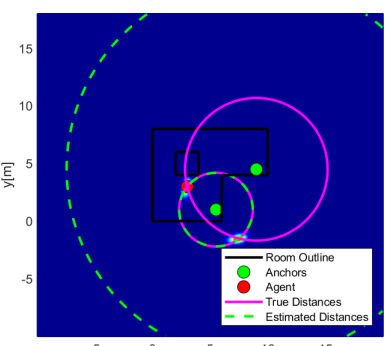

5
$x[m]$

Figure 4.15: The probability distributions for the dynamic error models $C$, $C S, D, D S$ and at position 1 for low SNR, i.e., $N_{0}=10^{-6}$. 

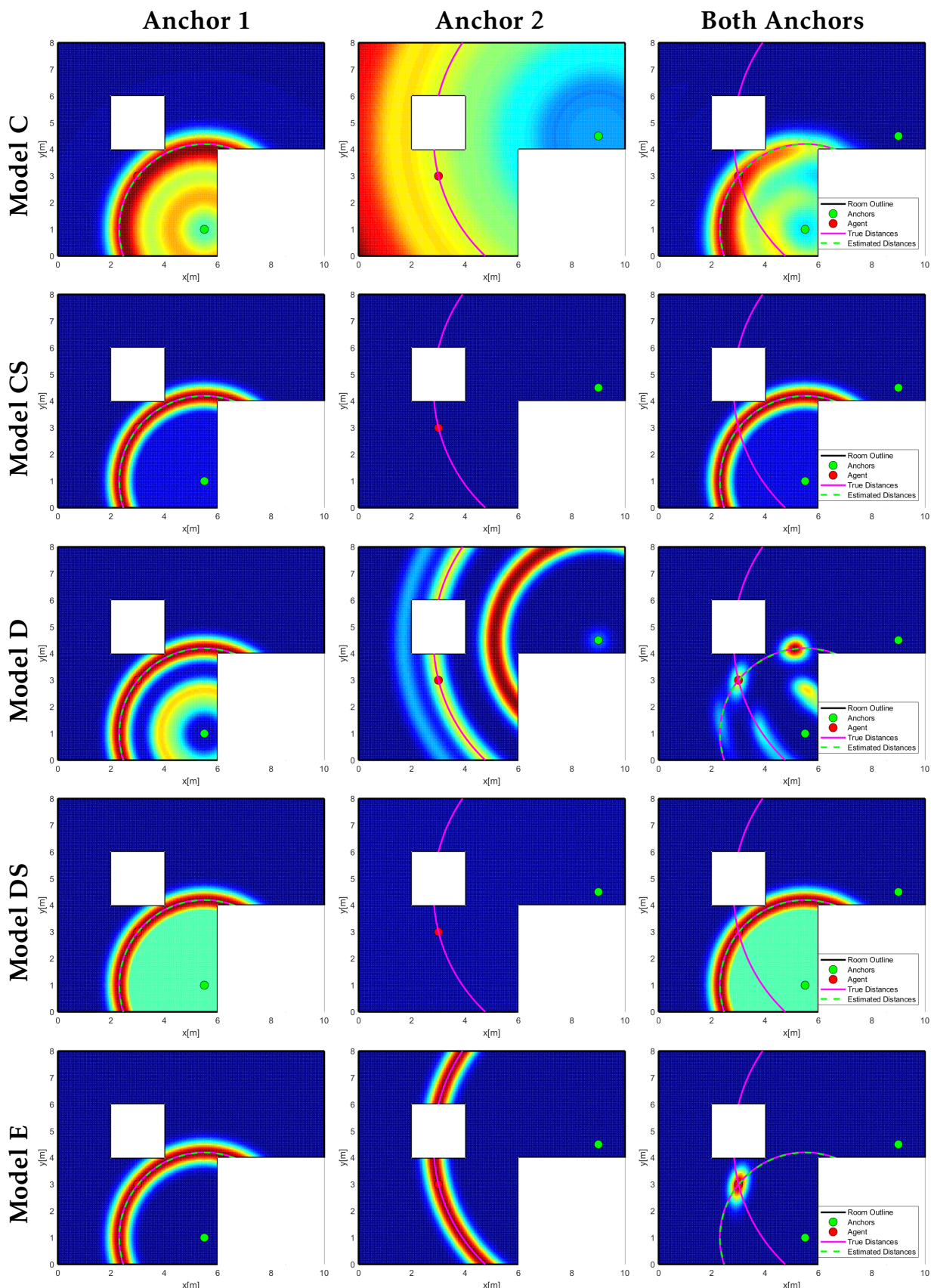

Figure 4.16: Same as Figure 4.15, but constrained to points within the room. 


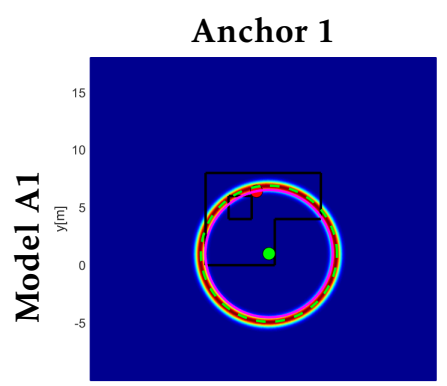

$x[\mathrm{~m}]$
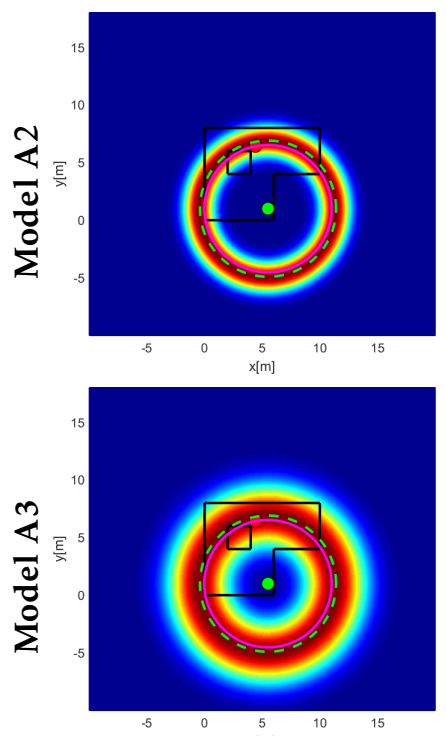

$x[\mathrm{~m}]$

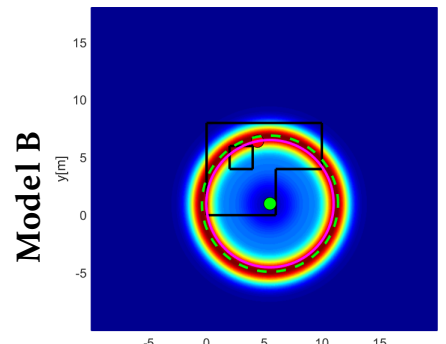

$\mathrm{x}[\mathrm{m}]$

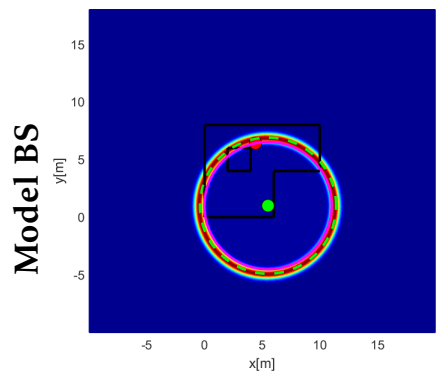

Anchor 2

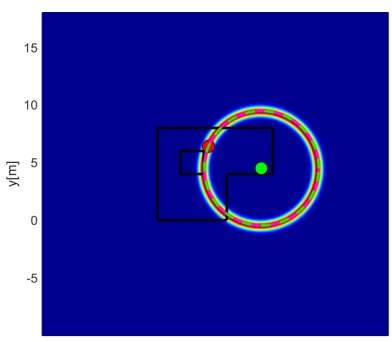

$x[\mathrm{~m}]$

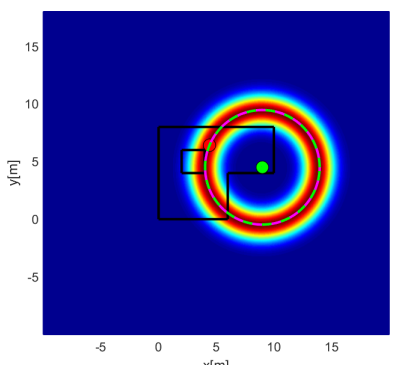

$x[\mathrm{~m}$

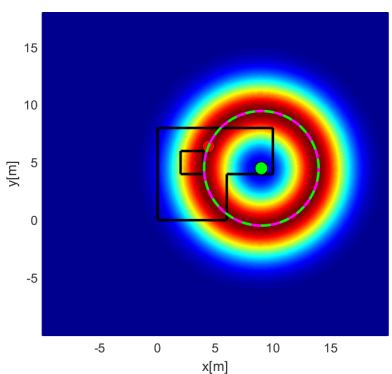

$x[m$

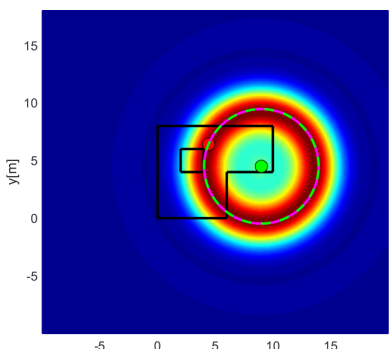

5
$x[\mathrm{~m}]$

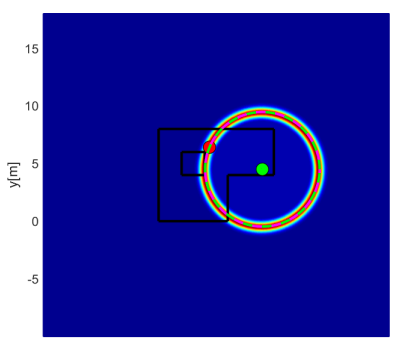

5
$x[\mathrm{~m}]$
Both Anchors

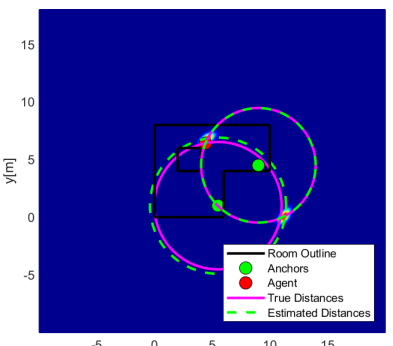

$\mathrm{x}[\mathrm{m}]$

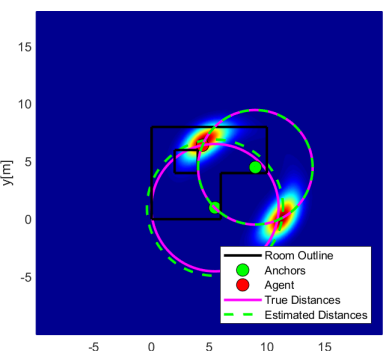

$\mathrm{x}[\mathrm{m}]$
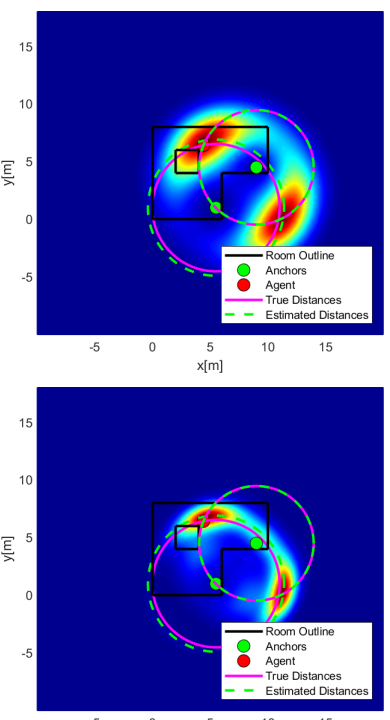

$x[\mathrm{~m}]$

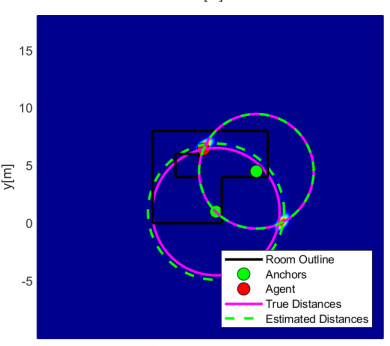

5
$x[\mathrm{~m}]$

Figure 4.17: The probability distributions for the static error models $A 1, A 2$, $A 3, B$ and $B S$ at position 136 for low SNR, i.e., $N_{0}=10^{-6}$. 

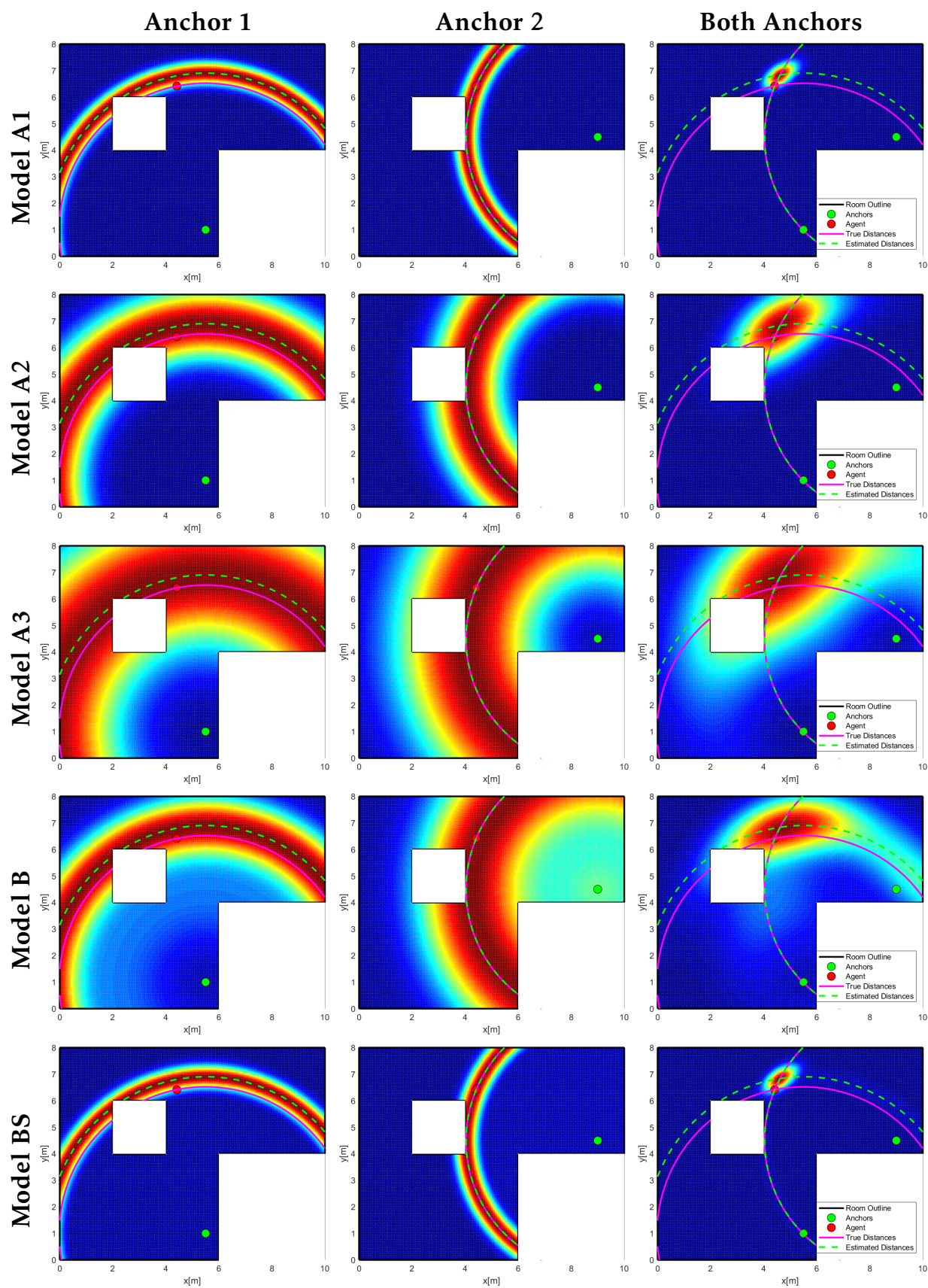

Figure 4.18: Same as Figure 4.17, but constrained to points within the room. 


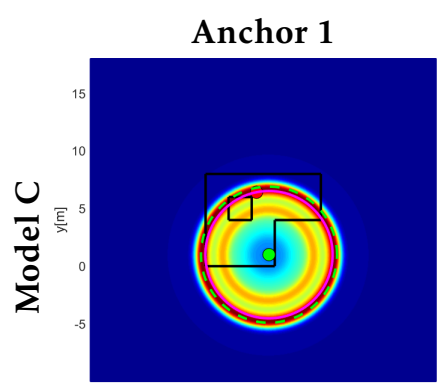

$x[m]$
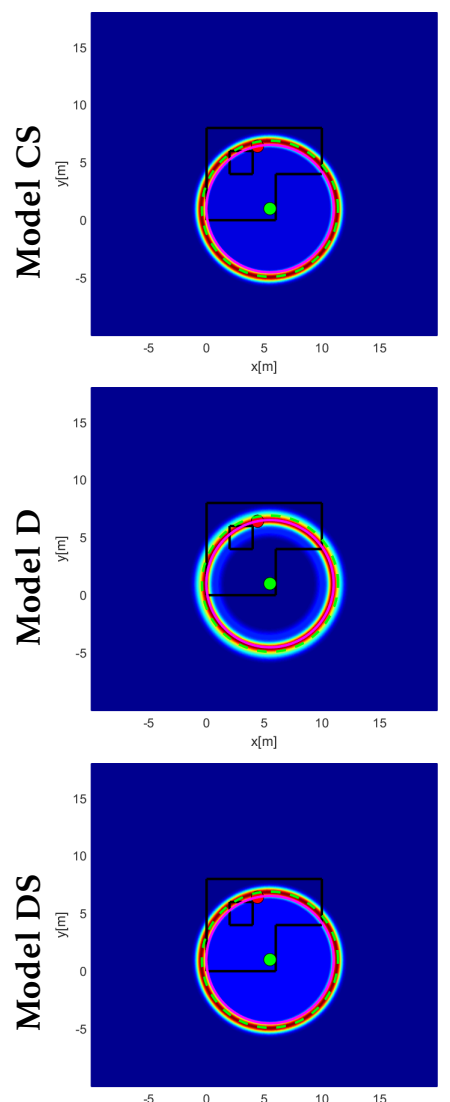

$\mathrm{x}[\mathrm{m}]$

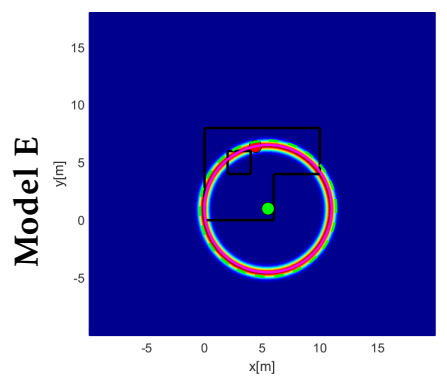

Anchor 2

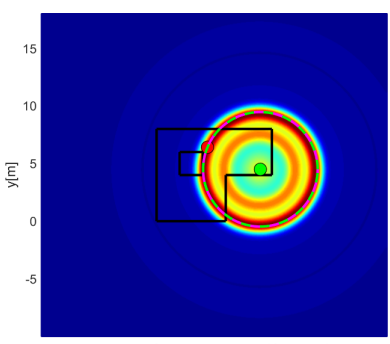

$x[\mathrm{~m}]$

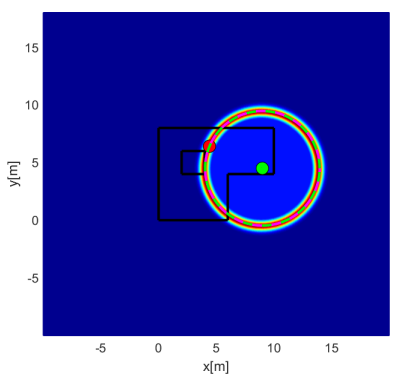

$x[\mathrm{~m}]$

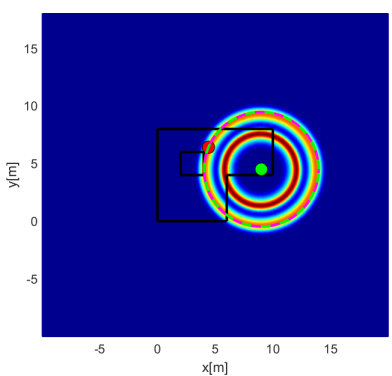

$x[\mathrm{~m}]$

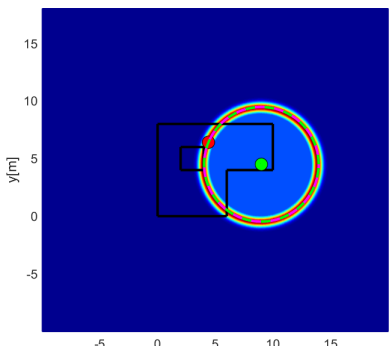

$x[\mathrm{~m}]$

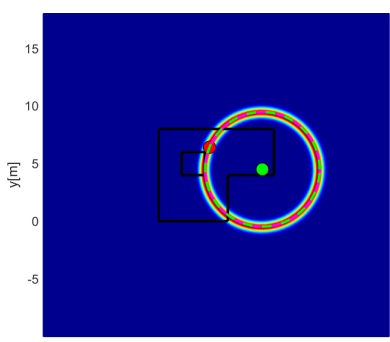

5
$x[\mathrm{~m}]$
Both Anchors

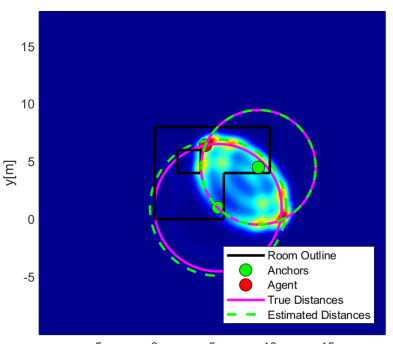

$\mathrm{x}[\mathrm{m}]$
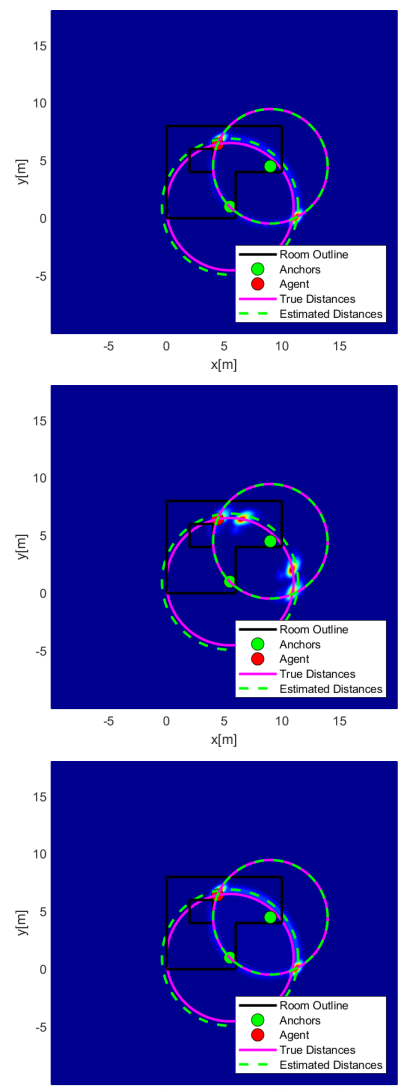

$x[\mathrm{~m}]$

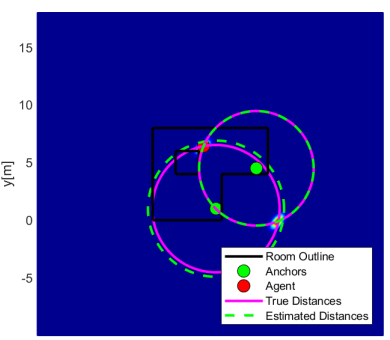

5
$x[\mathrm{~m}]$

Figure 4.19: The probability distributions for the dynamic error models $C$, $C S, D, D S$ and at position 136 for low SNR, i.e., $N_{0}=10^{-6}$. 

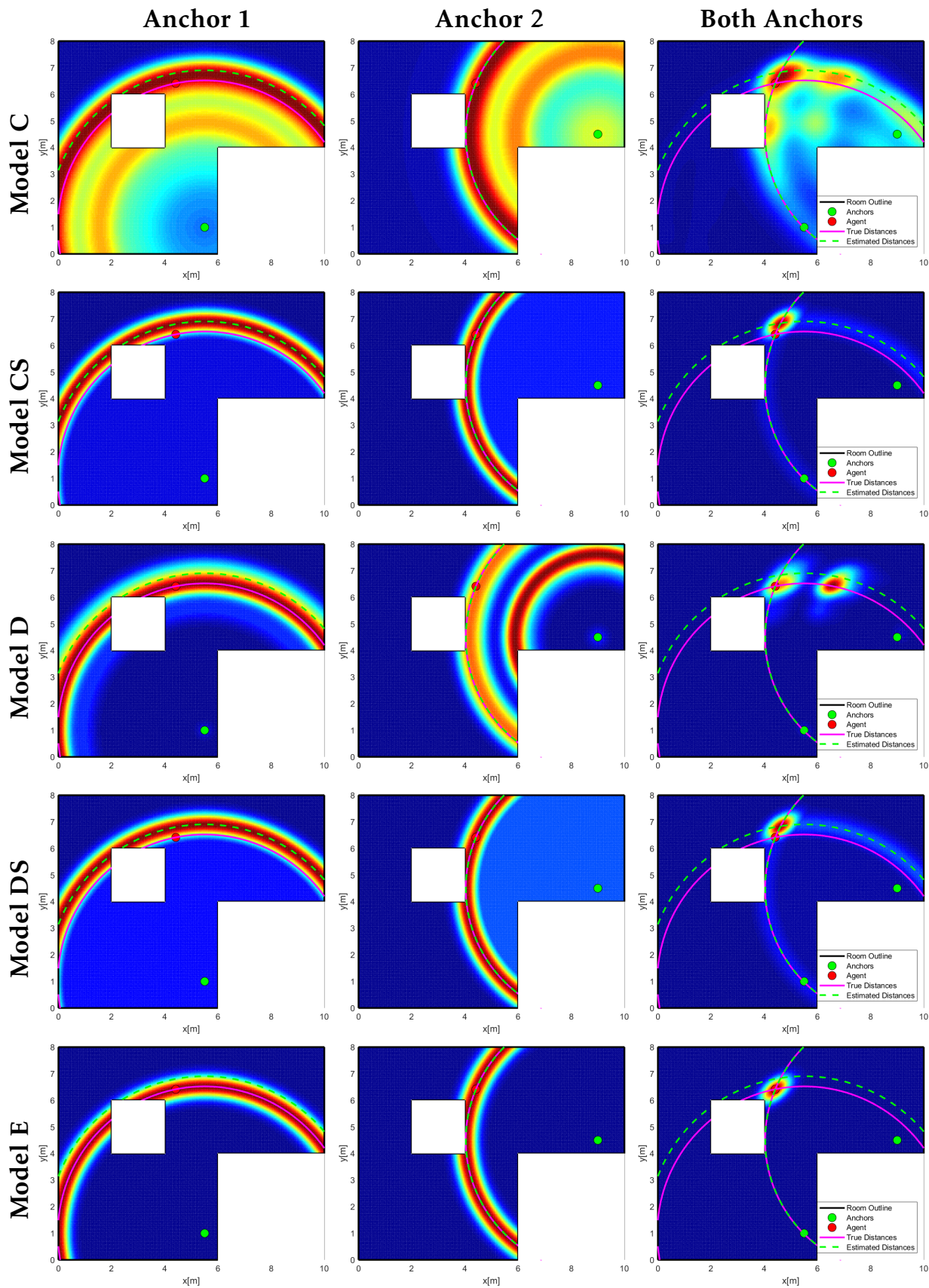

Figure 4.20: Same as Figure 4.19, but constrained to points within the room. 

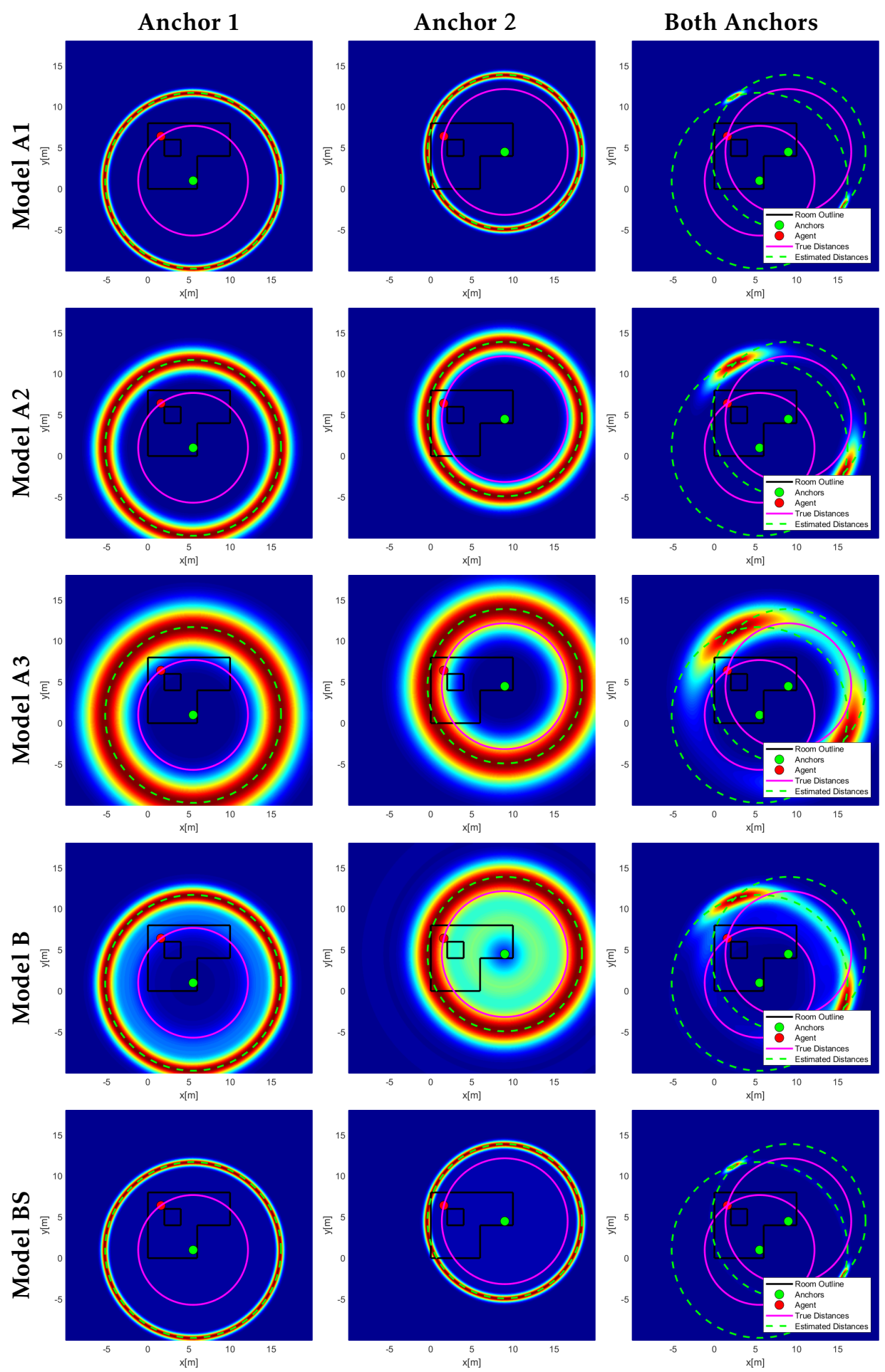

Figure 4.21: The probability distributions for the static error models $A 1, A 2$, $A 3, B$ and BS at position 136 for low SNR, i.e., $N_{0}=10^{-6}$. 

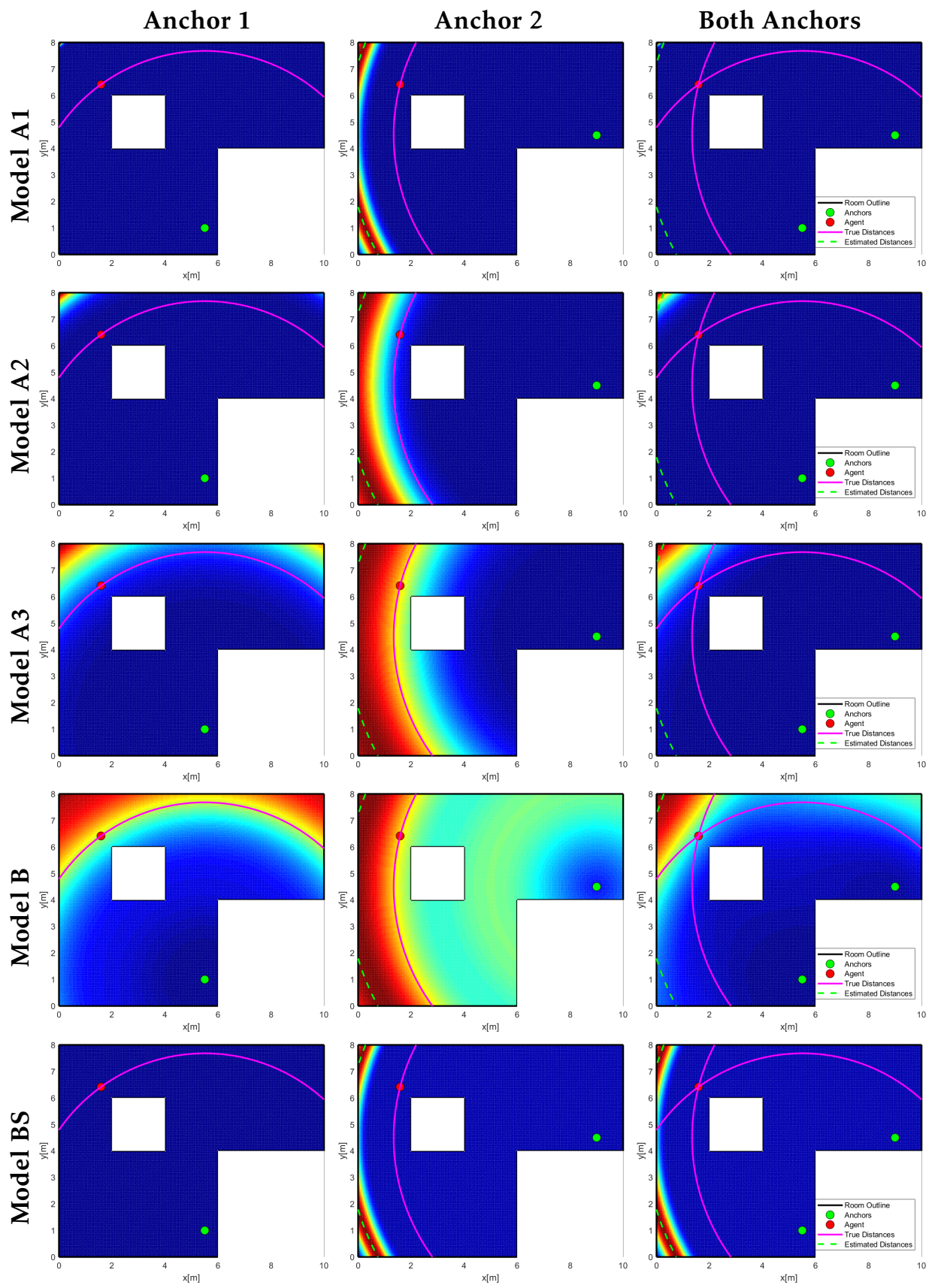

Figure 4.22: Same as Figure 4.21, but constrained to points within the room. 


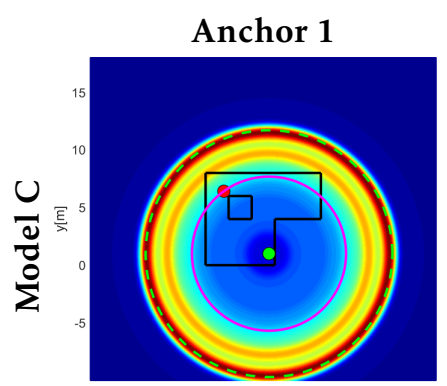

$x[m]$
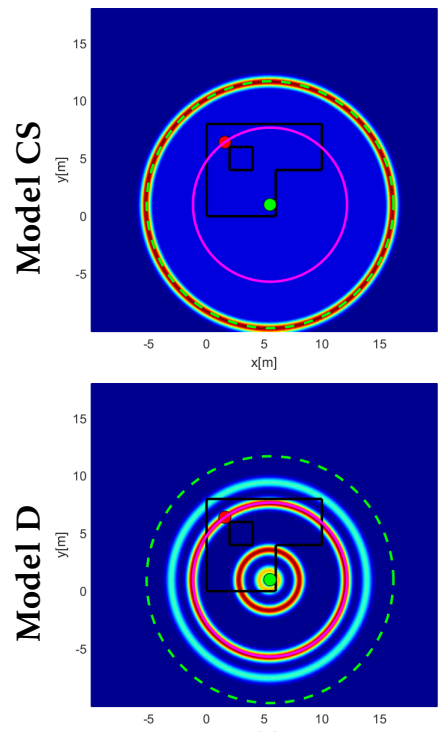

$x[\mathrm{~m}]$
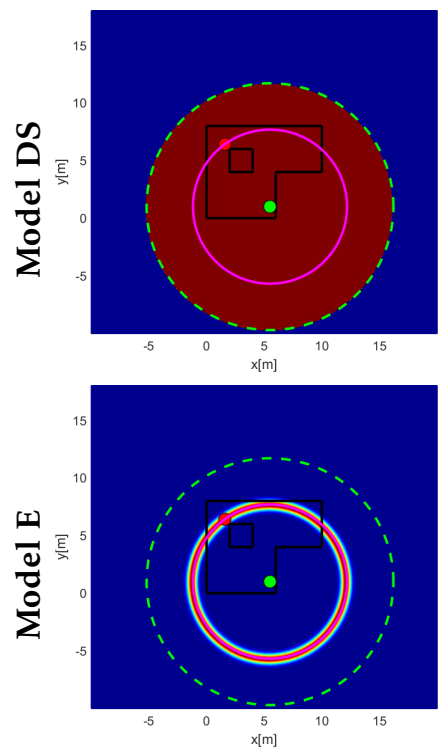

Anchor 2

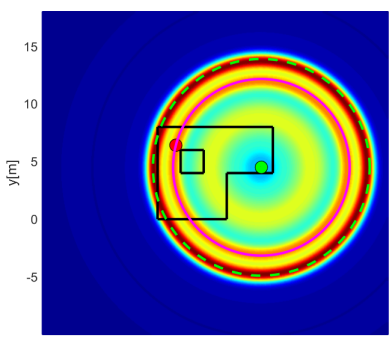

$x[\mathrm{~m}]$

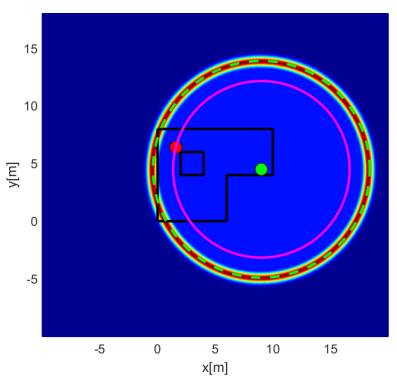

$x[\mathrm{~m}$
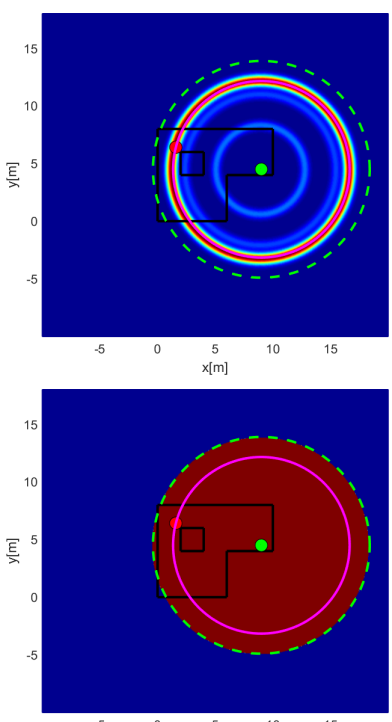

$\mathrm{x}[\mathrm{m}]$

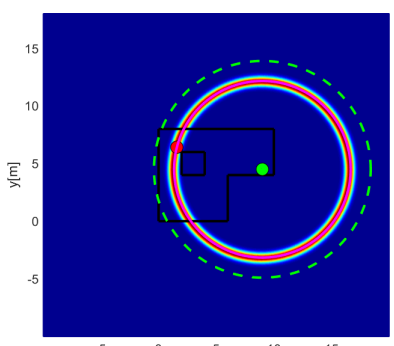

5
$x[m]$
Both Anchors
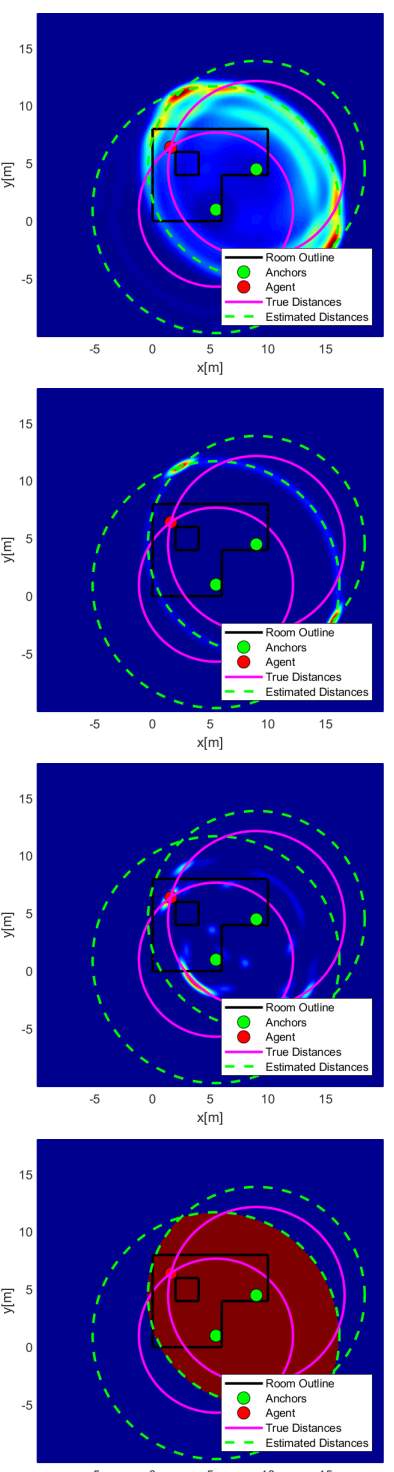

$\mathrm{x}[\mathrm{m}$

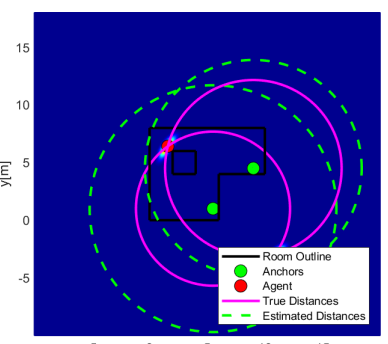

5
$x[\mathrm{~m}]$

Figure 4.23: The probability distributions for the dynamic error models $C$, $C S, D, D S$ and at position 136 for low SNR, i.e., $N_{0}=10^{-6}$. 

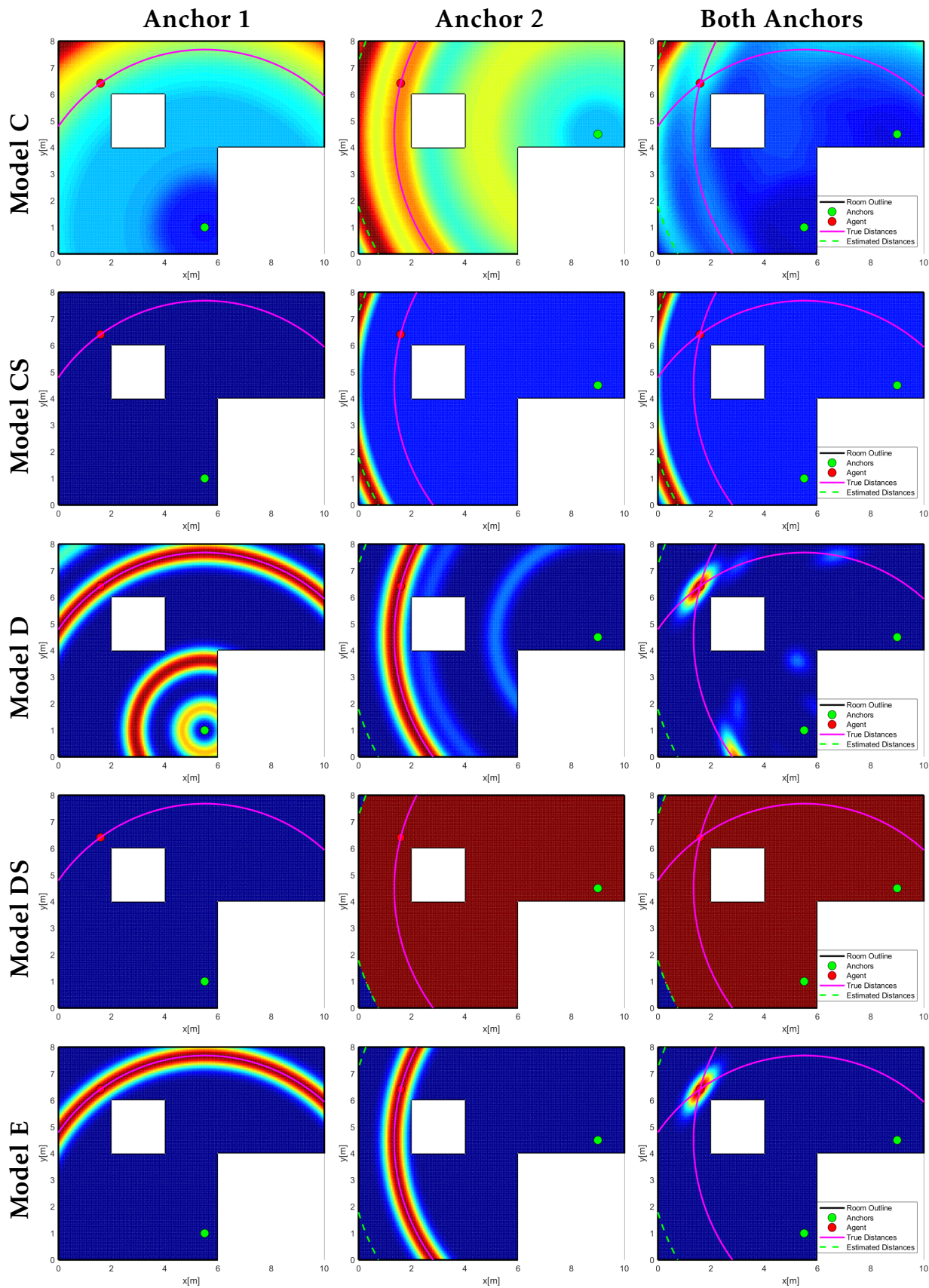

Figure 4.24: Same as Figure 4.23, but constrained to points within the room. 



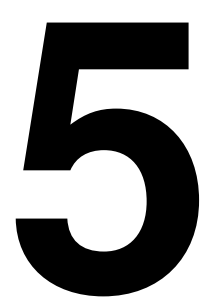

\section{The Measurements Association Problem}

This chapter deals with the topic on how to properly perform the association of measurements to the respective multi-path components of the radio channel.

This chapter is organized as follows: Section 5.1 formulates the problem, after which Section 5.2 discusses how to associate the available measurements with the respective MPCs, resulting in a vast amount of hypotheses, whereas Section 5.3 proposes how to reduce this number. Numerical results of these various aspects are presented in Section 5.4 whereafter a summary of the results is given in Section 5.5. Additional numerical results are presented in the appendix of Section 5.A.

\subsection{Problem Formulation}

Given the pseudo-range measurements of Chapter 3.3, there are non-ideal conditions which need to be catered for when associating these measurements $y_{m}^{(n)}$ for $n \in\left\{1, \ldots, N_{m}\right\}$ and a given anchor $m$ to the respective MPC/VA $k \in\left\{1, \ldots, K_{m}\right\}$. Ideally, this association is known, and we can without any loss of generality assume $k=n$ for all MPCs/VAs $k$ and measurements $n$. However:

- Perfect association of a given measurement $n$ with respect to the correct $\mathrm{MPC} / \mathrm{VA} k$ may not be feasible or even possible.

- A given MPC/VA $k$ may not be detected, and hence the corresponding measurement is missing - typically referred to as missed detections (MD).

- A given measurement $n$ may not be from a MPC/VA but rather due to, e.g., background noise or clutter — referred to as a false alarm (FA).

These topics need to be addressed in order to be able to proceed to the end goal, which is to acquire an as good estimate as possible of the agent position $\mathbf{x}$ as discussed in Section 3.4, which thus requires the forming of the corresponding PDF as discussed next. 


\subsection{Measurements Association}

We start by looking specifically at anchor $m$ and hence, for brevity in notation from here on (unless explicitly stated otherwise) we will omit the subscript $m$.

\subsubsection{Mapping from Measurements to MPCs/VAS}

We define the following mapping from the $N$ measurements to the $K$ MPCs/VAs:

$$
\theta: \underbrace{\{1, \ldots, N\}}_{\text {Measurements }} \rightarrow \underbrace{\{1, \ldots, K\}}_{\text {MPCs/VAs }} \cup \mathrm{FA}
$$

so that e.g. $\theta(n)=k$ means that measurement $n$ is associated with MPC/VA $k$ but $\theta(n)=$ FA means that measurement $n$ is a false alarm (FA). Further, we let $\theta^{-1}(k)=n$ mean that MPC/VA $k$ is measured by measurement $n$ and $\theta^{-1}(k)=\mathrm{MD}$ means that MPC/VA $k$ failed to be detected, i.e., a missed detection (MD), and hence is not covered by the measurements.

The set of MPCs/VAs associated with the $N$ measurements, disregarding the false alarms (FAs), are given by

$$
\mathcal{K}=\{\theta(n): n=1, \ldots, N\} \backslash \mathrm{FA} \subseteq \mathcal{K}^{\text {visible }} \subseteq\{1, \ldots, K\}
$$

where $\mathcal{K}^{\text {visible }}$ is defined (just as in (2.22)) as the subset of all $K$ MPCs/VAs that are visible at the agent. Similarly, the set of measurements associated with the $K$ MPCs/VAs are, disregarding the missed detections (MDs), is given by

$$
\mathcal{N}=\left\{\theta^{-1}(k): k=1, \ldots, K\right\} \backslash \mathrm{MD} \subseteq\{1, \ldots, N\}
$$

so $|\mathcal{K}| \leq\left|\mathcal{K}^{\text {visible }}\right| \leq K$ and $|\mathcal{N}| \leq N$, where $|\cdot|$ denotes the set cardinalities.

\subsubsection{Multiple Hypotheses}

The mapping $\theta$ of (5.1) is in all non-trivial cases unknown, but we can enumerate the $S$ different mapping hypotheses $\theta_{s}$ for $s=1, \ldots, S$.

Given $N$ number of measurements of which $n_{\mathrm{FA}}=0, \ldots, N$ are FAs, not taking into account the ordering of these FAs, there are $\left(\begin{array}{c}N \\ n_{\mathrm{FA}}\end{array}\right)$ possibilities of having precisely $n_{\mathrm{FA}}$ number of FAs. Furthermore, having $n_{\mathrm{FA}}$ FAs means that there are $N-n_{\mathrm{FA}}$ actual measurements of the $K$ MPCs/VAs and hence not FAs. Assuming that we do not measure the same MPC/VA twice and further also take into account the ordering of these measurement, the number of such measurement combinations are $\frac{K !}{\left(K-N+n_{\mathrm{FA}}\right) !}$. Hence, the total number of hypotheses depends on the number of measurements $N$ and MPCs/VAs $K$ as

$$
S(N, K)=\sum_{n_{\mathrm{FA}}=0}^{N} \frac{K !}{\left(K-N+n_{\mathrm{FA}}\right) !}\left(\begin{array}{c}
N \\
n_{\mathrm{FA}}
\end{array}\right)=\sum_{n_{\mathrm{FA}}=0}^{N}\left(\begin{array}{c}
N \\
n_{\mathrm{FA}}
\end{array}\right)^{N-n_{\mathrm{FA}}-1}(K-i)
$$




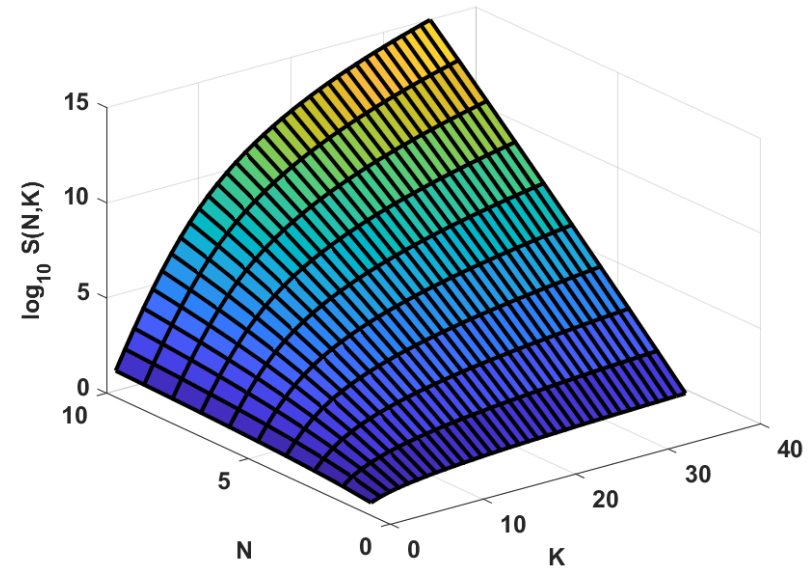

Figure 5.1: The number of possible hypothesis given by (5.4).

which grows combinatorially and very quickly for all but very small values of $K$ and $N$, as illustrated in Figure 5.1 above.

The PDF of the measurements $\mathbf{y}$ for a given anchor, given the agent position $\mathbf{x}$, the VA positions $\left\{\mathbf{p}^{(k)}\right\} \triangleq\left\{\mathbf{p}^{(k)}\right\}_{k=1}^{K}$ and the set of mapping hypotheses $\left\{\theta_{s}\right\}_{s=1}^{S}$ is given by the total probability theorem as

$$
p\left(\mathbf{y} \mid \mathbf{x},\left\{\mathbf{p}^{(k)}\right\}\right)=\sum_{s=1}^{S} p\left(\mathbf{y}, \theta_{s} \mid \mathbf{x},\left\{\mathbf{p}^{(k)}\right\}\right),
$$

where $p\left(\mathbf{y}, \theta_{s} \mid \mathbf{x},\left\{\mathbf{p}^{(k)}\right\}\right)$ is the joint PDF of the measurements $\mathbf{y}$ and the specific mapping hypotheses $\theta_{s}$ given the agent position $\mathbf{x}$ and the VA positions $\left\{\mathbf{p}^{(k)}\right\}$, which can be expressed as

$$
p\left(\mathbf{y}, \theta_{s} \mid \mathbf{x},\left\{\mathbf{p}^{(k)}\right\}\right)=p\left(\mathbf{y} \mid \mathbf{x},\left\{\mathbf{p}^{(k)}\right\}, \theta_{s}\right) p\left(\theta_{s} \mid \mathbf{x},\left\{\mathbf{p}^{(k)}\right\}\right) .
$$

The first factor of the right-hand side of (5.6) is in our model given by

$$
p\left(\mathbf{y} \mid \mathbf{x},\left\{\mathbf{p}^{(k)}\right\}, \theta_{s}\right)=\prod_{n \in \mathcal{N}_{s}} p\left(y^{(n)} \mid \mathbf{x}, \mathbf{p}^{\left(\theta_{s}(n)\right)}\right)
$$

where it has been utilized that the measurements are independent under the made assumptions and wherein the distribution of each such measurement is given by (3.14), but now considering the mapping hypothesis $\theta_{s}$ and the corresponding set of measurements $\mathcal{N}_{s}$.

The second factor of the right-hand side of (5.6) is the probability of the mapping $\theta_{s}$ which should cater for the joint probability of: 1) detecting the valid measurements in $\mathcal{N}_{s}, 2$ ) not detecting the MPCs/VAs not in the set $\mathcal{K}_{s}$ and 3) the false alarms (FAs) by measurements not in $\mathcal{N}_{s}$. Letting $P_{D}(k)$ denote the probability of detecting MPC/VA $k$ and $P_{F A}$ the probability of false alarms (FAs): 


$$
\begin{aligned}
p\left(\theta_{s} \mid \mathbf{x},\left\{\mathbf{p}^{(k)}\right\}\right) & =\left[\prod_{n \in \mathcal{N}_{S}} P_{D}\left(\theta_{s}(n)\right)\right]\left[\prod_{k \notin \mathcal{K}_{s}}\left(1-P_{D}(k)\right)\right] \prod_{n \notin \mathcal{N}_{s}} P_{F A} \\
& =\left[\prod_{n \in \mathcal{N}_{s}} \frac{P_{D}\left(\theta_{s}(n)\right)}{P_{\mathrm{FA}}\left(1-P_{D}\left(\theta_{s}(n)\right)\right)}\right] \prod_{k=1}^{K}\left(1-P_{D}(k)\right) \prod_{n=1}^{N} P_{\mathrm{FA}}
\end{aligned}
$$

where the sets $\mathcal{K}_{s}$ and $\mathcal{N}_{s}$ are defined as in (5.2) and (5.3) wrt. the mapping $\theta_{s}$.

Hence, with the help of (5.7) and (5.8) we can express (5.6) as

$$
p\left(\mathbf{y}, \theta_{s} \mid \mathbf{x},\left\{\mathbf{p}^{(k)}\right\}\right)=\left[\prod_{n \in \mathcal{N}_{s}} p\left(y^{(n)} \mid \mathbf{x}, \mathbf{p}^{\left(\theta_{s}(n)\right)} \frac{P_{D}\left(\theta_{\mathcal{S}}(n)\right)}{P_{\mathrm{FA}}\left(1-P_{D}\left(\theta_{\mathcal{S}}(n)\right)\right)}\right]\left[\prod_{k=1}^{K}\left(1-P_{D}(k)\right)\right] \prod_{n=1}^{N} P_{\mathrm{FA}} .\right.
$$

By now summing (5.9) over all possible hypothesis as per (5.5) we get the total probability distribution which can subsequently be used to estimate the agent position $\mathbf{x}$ as discussed in Section 3.4.

\subsection{Hypothesis Reduction}

The resulting probabilities of (5.9) requires the calculation of an, in most cases, extremely large number of possible hypotheses $S$ as per (5.4) and Figure 5.1. This section aims at reducing this complexity by finding the most likely hypothesis/es and also reducing the number of measurements which need to be considered.

\subsubsection{Finding the Best Hypothesis}

Finding the best (most probable) hypothesis mapping, denoted $\theta^{*}$, can be done by solving the well-known assignment problem (see e.g. [5], [3]). We assume, as stated previously, that each measurement $n$ can be associated to at most one $\mathrm{MPC} / \mathrm{VA} k$ and conversely that each MPC/VA can be associated to at most one measurement. This boils down to solving the following optimization problem:

$$
\begin{array}{ll}
\text { minimize } & C(\mathbf{Q}, \mathbf{R})=\sum_{n=1}^{N} \sum_{k=1}^{K}[\mathbf{Q}]_{n k}[\mathbf{R}]_{n k} \\
\text { subject to } & \sum_{k=1}^{K}[\mathbf{R}]_{n k} \leq 1 \text { for } n=1, \ldots, N \\
& \sum_{n=1}^{N}[\mathbf{R}]_{n k} \leq 1 \text { for } k=1, \ldots, K
\end{array}
$$

where $\mathbf{Q} \in \mathbb{R}^{N \times K}$ is the association matrix with elements $[\mathbf{Q}]_{n k} \in \mathbb{R}, \mathbf{R} \in\{0,1\}^{N \times K}$ is a binary matrix with elements $[\mathbf{R}]_{n k} \in\{0,1\}$ and $C(\mathbf{Q}, \mathbf{R})$ is the therewith associated total cost. 
To form the association matrix $\mathbf{Q}$ in (5.10), we take the negative logarithm of the per-hypothesis PDF $p\left(\mathbf{y}, \theta_{s} \mid \mathbf{x},\left\{\mathbf{p}^{(k)}\right\}\right)$ in (5.9) and make use of (3.15):

$$
\begin{aligned}
-\ln p\left(\mathbf{y}, \theta_{s} \mid \mathbf{x},\left\{\mathbf{p}^{(k)}\right\}\right)= & \sum_{n \in \mathcal{N}_{s}}\left(-\ln p\left(y^{(n)} \mid \mathbf{x}, \mathbf{p}^{\left(\theta_{s}(n)\right)}\right)-\ln \frac{P_{D}\left(\theta_{s}(n)\right)}{P_{\mathrm{FA}}\left(1-P_{D}\left(\theta_{s}(n)\right)\right)}\right) \\
& -\sum_{k=1}^{K} \ln \left(1-P_{D}(k)\right)-\sum_{n=1}^{N} \ln P_{\mathrm{FA}} \\
= & \sum_{n \in \mathcal{N}_{s}}\left(\frac{1}{2} \ln 2 \pi \sigma_{\theta_{s}(n)}^{2}+\frac{\left(y^{(n)}-\left\|\mathbf{x}-\mathbf{p}^{\left(\theta_{s}(n)\right)}\right\|_{2}\right)^{2}}{2 \sigma_{\theta_{s}(n)}^{2}}\right. \\
& \left.\quad-\ln \frac{P_{D}\left(\theta_{s}(n)\right)}{P_{\mathrm{FA}}\left(1-P_{D}\left(\theta_{s}(n)\right)\right)}\right)-\sum_{k=1}^{K} \ln \left(1-P_{D}(k)\right)-\sum_{n=1}^{N} \ln P_{\mathrm{FA}} .
\end{aligned}
$$

One can interpret the expression (5.11) as the total cost of assigning the $N$ different measurements to the $K$ different MPCs/VAs, assuming the mapping $\theta_{s}$. The last two sums in this expression are the same for all choices of $\theta_{s}$ and can hence be treated as constants when solving (5.10), since they will not influence the end result. Given this insight, we now continue to form the elements of $\mathbf{Q}$ as

$$
[\mathbf{Q}]_{n k}=\frac{1}{2} \ln 2 \pi \sigma_{k}^{2}+\frac{\left(y^{(n)}-\left\|\mathbf{x}-\mathbf{p}^{(k)}\right\|_{2}\right)^{2}}{2 \sigma_{k}^{2}}-\ln \frac{P_{D}(k)}{P_{\mathrm{FA}}\left(1-P_{D}(k)\right)}
$$

which thus describes the cost of assigning measurement $n$ to MPC/VA $k$.

Letting $\mathbf{R}^{*}$ denote the optimal solution to (5.10), the corresponding optimal or best mapping hypothesis $\theta^{*}$ is then given by

$$
\theta^{*}(n)=\left\{\begin{array}{ll}
k & \text { if } \exists k:\left[\mathbf{R}^{*}\right]_{n k}=1 \\
\text { FA } & \text { otherwise }
\end{array} \text { for } n=1, \ldots, N .\right.
$$

\subsubsection{Finding the B-Best Hypotheses}

We can also find not only the optimal/best solution $\mathbf{R}^{*}$ to the assignment problem (5.10) with the corresponding mapping $\theta^{*}$ as discussed in the previous subsection, but rather the set of the $B \leq S$ best solutions $\left\{\mathbf{R}_{b}^{*}\right\}$ for $b=1, \ldots, B$. We order these by increasing cost $C\left(\mathbf{Q}, \mathbf{R}_{b}^{*}\right)$ so that $\mathbf{R}_{1}^{*}$ is the best solution (i.e., same as $\mathbf{R}^{*}$ in the previous subsection), $\mathbf{R}_{2}^{*}$ is the second-best solution etc.

For each solution, we denote the mapping hypothesis $\theta_{b}^{*}$ with the corresponding probabilities $p\left(\mathbf{y}, \theta_{b}^{*} \mid \mathbf{x},\left\{\mathbf{p}^{(k)}\right\}\right)$ as per (5.9), which allows us for a sufficiently large value of $B$ to approximate (5.9) as

$$
p\left(\mathbf{y} \mid \mathbf{x},\left\{\mathbf{p}^{(k)}\right\}\right)=\sum_{s=1}^{S} p\left(\mathbf{y}, \theta_{s} \mid \mathbf{x},\left\{\mathbf{p}^{(k)}\right\}\right) \approx \sum_{b=1}^{B} p\left(\mathbf{y}, \theta_{b}^{*} \mid \mathbf{x},\left\{\mathbf{p}^{(k)}\right\}\right) \triangleq p^{B}\left(\mathbf{y} \mid \mathbf{x},\left\{\mathbf{p}^{(k)}\right\}\right)
$$


which, given all $S$ hypotheses, would give an equality in (5.14), i.e.,

$$
p^{S}\left(\mathbf{y} \mid \mathbf{x},\left\{\mathbf{p}^{(k)}\right\}=p\left(\mathbf{y} \mid \mathbf{x},\left\{\mathbf{p}^{(k)}\right\}\right) .\right.
$$

\subsubsection{Gating of Measurements}

Now, as commented earlier, the number of hypotheses $S$ can be quite significant. To further keep the complexity down and make the computations more feasible, we gate the measurements (see [5]) in the sense that we consider only entries $n, k$ in the cost matrix $\mathbf{Q}$ of (5.12) such that the corresponding pseudo-range measurements $y^{(n)}$ are within 3 standard deviations of the distance, $\left\|\mathbf{x}-\mathbf{p}^{(k)}\right\|_{2}$ to the respective VA $k$. The resulting, gated cost matrix $\mathbf{Q}^{G}$ hence has entries

$$
\mathbf{Q}_{n k}^{G}= \begin{cases}\mathbf{Q}_{n k} \text { as per (5.12) } & \text { if }\left|y^{(n)}-\left\|\mathbf{x}-\mathbf{p}^{(k)}\right\|_{2}\right| \leq 3 \sigma_{k} \\ \infty & \text { otherwise }\end{cases}
$$

which means that any measurement $n$ not passing this gate for any MPC/VA, will be associated with an infinite cost and not considered in the assignment.

\subsection{Numerical Results}

Evaluations are performed for Scenario A presented in Section 2.1 with two different agent positions, just as per Example 2.1 in Chapter 2. The essential scenario parameters are given by Table 5.1. The evaluations are done using 50 MonteCarlo (MC) realizations over a $5 \times 5 \mathrm{~cm}$ uniform grid.

Table 5.1: Scenario Parameters

\begin{tabular}{|c|c|c|c|}
\hline Parameter & Value & Description & Comment \\
\hline $\mathbf{x}_{1}$ & {$[3,3]^{T}$} & Agent Position 1 & Unknown \\
$\mathbf{x}_{2}$ & {$[1,7]^{T}$} & Agent Position 2 & Unknown \\
$\mathbf{p}_{1}$ & {$[5.5,1.0]^{T}$} & Anchor 1 Position & Known \\
$\mathbf{p}_{2}$ & {$[9.0,4.5]^{T}$} & Anchor 2 Position & Known \\
$\max Q^{(k)}$ & 3 & Max MPC Order & Max no reflections \\
$d_{\mathrm{MAX}}$ & $30 \mathrm{~m}$ & Max Measurement Distance & - \\
\hline
\end{tabular}

\subsubsection{Measurement Assumptions}

The resulting received power versus the range for the MPCs of the considered scenario, i.e., $\left\{\left|\alpha_{m}^{(k)}\right|^{2}\right\}$ and $\left\{d_{m}^{(k)}\right\}=\left\{c \tau_{m}^{(k)}\right\}$ as discussed in Chapter 2, are illustrated in Figure 5.2. For each agent position, we generate several realizations of the these ideal pseudo-range measurements with respect to each visible VA of both anchors, i.e., according to $\mathcal{K}^{\text {visible }}$. 
Anchor 1
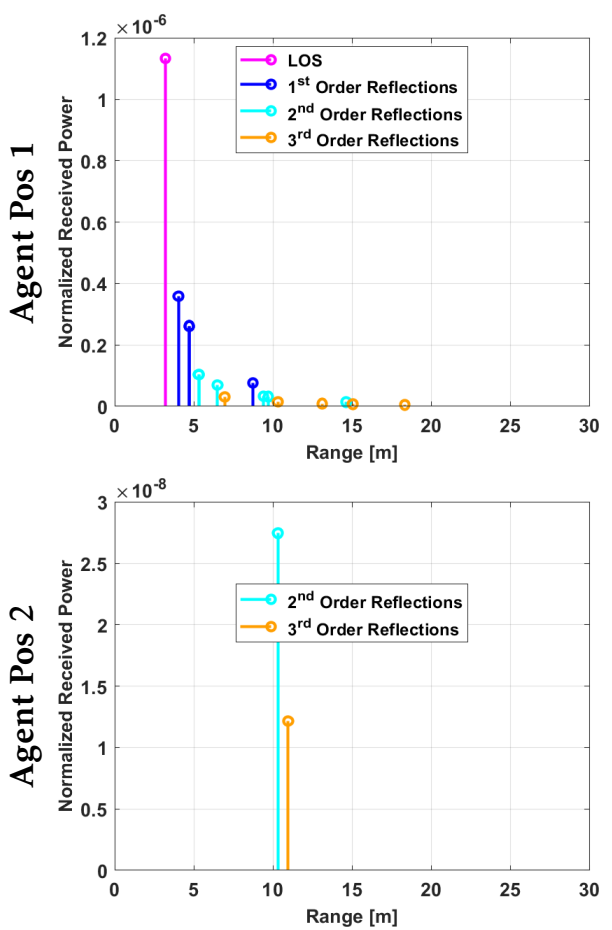

Anchor 2
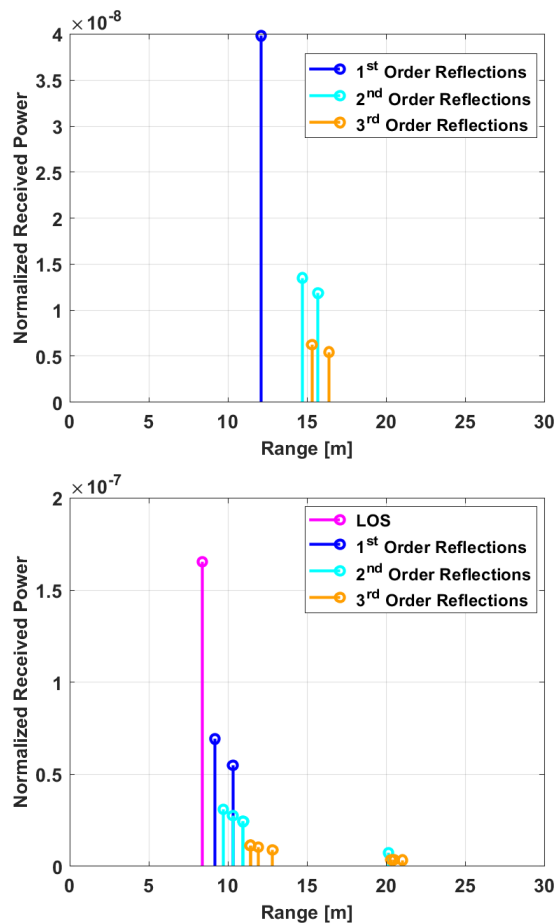

Figure 5.2: The received power versus range for the respective MPC on Scenario $A$ as in Example 2.1.

These measurements are generated under increasingly more complex assumptions as per Table 5.2. Note that we here use a value of $\sigma_{m, k}=0.2$ meters, which corresponds to $0.2 * 10^{9} / c \approx 0.7$ ns which is slightly smaller than the value of 1 ns used in the preceding Chapter 4 . This ought however to make no significant difference in terms of the quantitative results and reasoning of this chapter.

Table 5.2: Measurement Assumptions

\begin{tabular}{|c|l|ccc|}
\hline Assumption & Description & \multicolumn{3}{|c|}{ Parameter } \\
& & $\sigma_{m, k}$ & $P_{D}(k)$ & $\beta$ \\
\hline I & Ideal & 0 & 1 & 0 \\
II & Assumption I + Noise & 0.2 & 1 & 0 \\
III & Assumption II + Missed & 0.2 & $(5.17)$ & 0 \\
& Detections & & & \\
IV & Assumption III + False Alarms & 0.2 & $(5.17)$ & $2 / d_{\mathrm{MAX}}$ \\
\hline
\end{tabular}

For assumptions III and IV, the probability of detecting MPC/VA $k$, i.e., $P_{D}(k)$, is defined in a similar manner as in [30] depending on the number of reflections 
$Q^{(k)}$ and the visibility of the MPC/VA as

$$
P_{D}(k)=\left\{\begin{array}{lll}
0.8 & Q^{(k)}=0 \text { and } k \in \mathcal{K}^{\text {visible }} & \text { (LOS) } \\
0.5 & Q^{(k)}=1 \text { and } k \in \mathcal{K}^{\text {visible }} & \text { (Single Reflections) } \\
0.3 & Q^{(k)}=2 \text { and } k \in \mathcal{K}^{\text {visible }} & \text { (Double Reflections) } \\
0.1 & Q^{(k)}=3 \text { and } k \in \mathcal{K}^{\text {visible }} & \text { (Triple Reflections) } \\
0 & Q^{(k)} \geq 4 \text { or } k \notin \mathcal{K}^{\text {visible }} & \text { (Higher Order Reflections or non-LOS) }
\end{array}\right.
$$

where the numbers are chosen rather arbitrarily. Compared to [30], triple reflections have been added in (5.17). Note that $P_{D}(k)$ implicitly depends on both the agent position $\mathbf{x}$ and the VA positions $\left\{\mathbf{p}^{(k)}\right\}$ since these determine the number of reflections and hence $\mathcal{K}^{\text {visible }}$ as per (2.22) of Section 2.2. It should be pointed out that (5.17) still is a quite simple model, where a more realistic model would, e.g., take into account the amplitudes $\left\{\alpha^{(k)}\right\}$ and/or the signal-to-interferenceand-noise (SINR) of the respective MPC as done in Chapter 3 of this thesis.

For assumption IV, the number of false alarms (FAs) for each measurement realization is assumed to be Poisson distributed with intensity $\beta d_{\text {MAX }}$ whereas the related measurements are uniformly distributed over the range $\left[0, d_{\mathrm{MAX}}\right]$. The parameter $d_{\text {MAX }}$ here denotes the assumed maximum measurement distance with a value as per Table 5.1, wherein also the parameter $\beta$ is defined. Note that this model of the false alarms is also a simplified one whereas a more realistic model as in Chapter 3 would e.g. account for the power spectral density (PSD) of the background noise.

For illustrative purposes, histograms over the measurements with respect to anchor 1 when the agent is at $\mathbf{x}_{1}=[3,3]^{T}$ are shown in Figure 5.3.

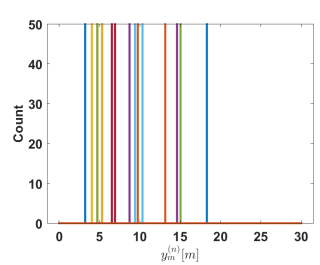

(a) Assumption I

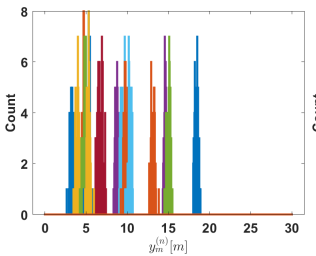

(b) Assumption II

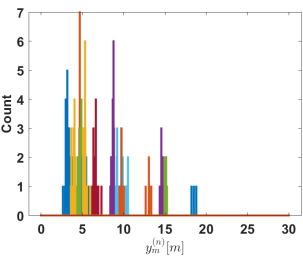

(c) Assumption III

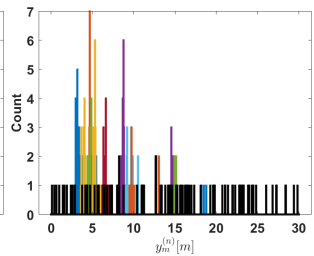

(d) Assumption IV

Figure 5.3: Histogram over the generated measurements wrt. anchor 1 when the agent is at $\mathbf{x}_{1}$ for the different assumptions I-IV of Table 5.2. The colour coding indicates the different VAs/MPCs where black is clutter/FAs.

\subsubsection{Estimation Models}

We start with a simple and idealized estimation model, denoted 'Model A', where:

- All MPCs/VAs are detectable, i.e., $P_{D}(k)=1 \forall k$.

- The mapping $\theta$ of (5.1) is known and need hence not be estimated.

- There are no false alarms, i.e., $P_{F A}=0$.

- Which MPCs/VAs that are visible, i.e., $\mathcal{K}^{\text {visible }}$ is known. 
Table 5.3: Estimation Models

\begin{tabular}{|c|c|cccc|}
\hline Model & Complexity & \multicolumn{4}{|c|}{ Parameter } \\
& & $P_{D}(k)$ & $\theta$ & $P_{\text {FA }}$ & $\mathcal{K}^{\text {visible }}$ \\
\hline A & Low (Ideal Model) & 1 & Known & 0 & Known \\
B & $\downarrow$ & $(5.17)$ & Known & 0 & Known \\
C & $\downarrow$ & $(5.17)$ & Unknown & 0 & Known \\
D & $\downarrow$ & $(5.17)$ & Unknown & 0.1 & Known \\
E & $\downarrow$ & $(5.17)$ & Unknown & 0 & Unknown \\
F & High (Full Model) & $(5.17)$ & Unknown & 0.1 & Unknown \\
\hline
\end{tabular}

Upon this model we gradually and stepwise add complexity until reaching the full model derived in the previous sections, denoted 'Model F', where:

- Not all MPCs/VAs are detectable, i.e., $P_{D}(k)<1$ as given by (5.17).

- The mapping $\theta$ of (5.1) is unknown and must therefore be estimated.

- There are false alarms present, i.e., $P_{F A}>0$.

- Which MPCs/VAs that are visible, i.e., $\mathcal{K}^{\text {visible }}$ is unknown, wherefore the estimation need to be done over all $K$ MPCs/VAs.

All-in-all, we define six different estimation models as per Table 5.3 above. For all models, it is assumed that the room layout is known, wherefore only points within the room are considered in the estimation process.

For models $\mathrm{C}$ through $\mathrm{F}$, wherein the mapping $\theta$ in (5.1) between measurements and MPCs/VAs is unknown, to find the single best hypothesis as discussed in Section 5.3.1 we need to solve (5.10). For this purpose we use a Global Nearest Neighbour (GNN) association algorithm - and more specifically the JonkerVolgenant (JV) algorithm (see e.g. [5, 18]). To find the B-Best hypotheses as discussed in Section 5.3.2 we additionally use Murty's algorithm (see [5, 34]).

We introduce the notations $p_{Q \mid R}$ and $\hat{\mathbf{x}}_{Q \mid R}$ to indicate that the probability calculation and estimation, respectively, are performed using estimation model $Q \in\{A, B, C, D, E, F\}$ based on measurements generated under the conditions of measurement model $R \in\{I, I I, I I I, I V\}$ as defined in the previous subsection. For each such case, the probability calculation and subsequent estimation is done using a grid search over the entire room.

\subsubsection{Approximation by using the Single Best Hypothesis}

When the true mapping $\theta$ between measurements and MPCs/VAs is unknown, the first relevant question is how good approximation that is possible without the need to evaluate all $S$ hypotheses, but rather only one or a subset of these as discussed in Sections 5.3.1 and 5.3.2. More precisely, we investigate how good an approximation the best hypothesis as discussed in Section 5.3.1 (i.e., $p\left(\mathbf{y}, \theta^{*} \mid \mathbf{x},\left\{\mathbf{p}^{(k)}\right\}\right)$ of (5.9)) is compared to the true one which is approximated as discussed in Section 5.3 .2 (i.e., $p^{B}\left(\mathbf{y} \mid \mathbf{x},\left\{\mathbf{p}^{(k)}\right\}\right)$ of (5.14)). For the latter we generate the $B=50$ best hypotheses - a limiting number chosen to make the computations feasible. The 
most general estimation model $F$ and measurement assumptions III (without FAs) and IV (with FAs) are used. The resulting probability plots for one single MC realization are shown for the anchors individually in Figures 5.4, 5.5 whereas jointly for both anchors in Figure 5.6. We make the following observations:

- For the individual anchor measurements of Figures 5.4 and 5.5, the probability of the best hypothesis align well with the sum over all hypotheses. This is particularly true for measurement assumption IV with FAs.

- For the individual anchor measurements using measurement assumption III, the discrepancy is larger - in particular for the less rich radio environments with few MPCs (i.e., agent position 2 using anchor 1 in Figure 5.4 and agent position 1 using anchor 2 in Figure 5.5). This is most likely because (as seen e.g. in Figure 5.2) there are fewer measurements available in this case, something which in turn makes the different mapping hypotheses become more diverse - there is a large ambiguity of which measurement that should be mapped to which MPC/VA - wherefore it becomes important to include several of them in order to get a good approximation of the resulting probability distributions. In contrast, in a richer radio environment with many measured MPCs/VAs, the ambiguity in the mapping process becomes smaller so that the majority of the hypotheses will give approximately the same probability distributions.

- Combining measurements from both anchors, as in Figure 5.6 the match between the best hypothesis and the sum over all hypotheses is quite good. Again, just as for the richer radio environments discussed above, this is due to the increased amount of measured MPCs/VAs to hypothesize over makes the individual hypotheses more similar.

\section{Agent Pos 1 - III}

Agent Pos 1 - IV

Agent Pos 2 - III

Agent Pos 2 - IV
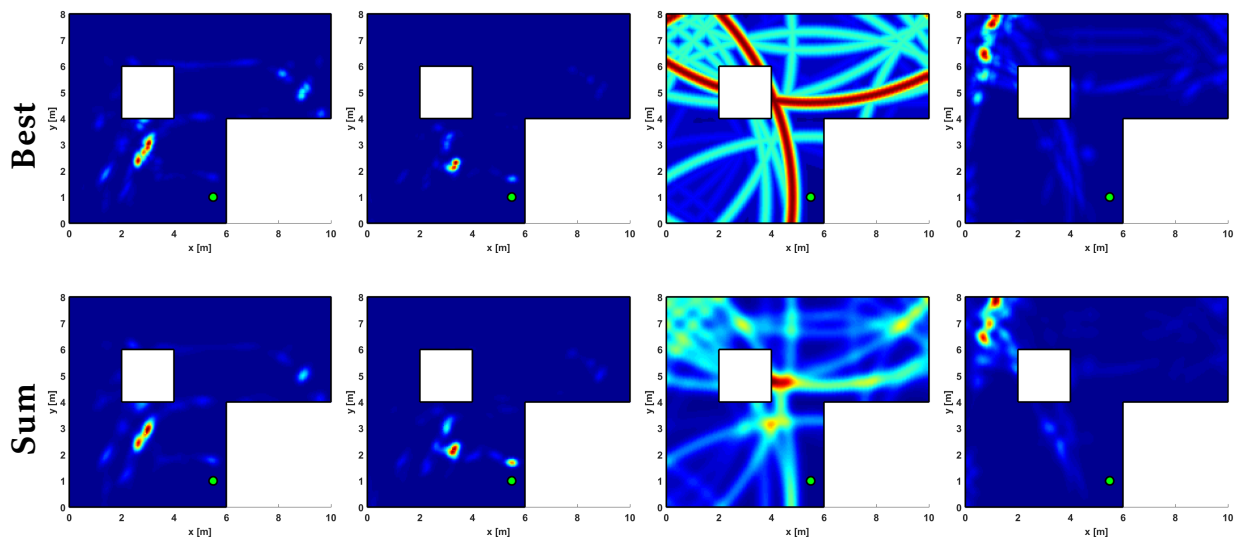

Figure 5.4: The probabilities $p_{F \mid R}$ for measurement assumptions $R \in$ $\{I I I, I V\}$ and one single MC realization for the best hypothesis (top row) and for the sum of all hypotheses (bottom row) for measurements of anchor 1. 
Agent Pos 1 - III Agent Pos 1 - IV
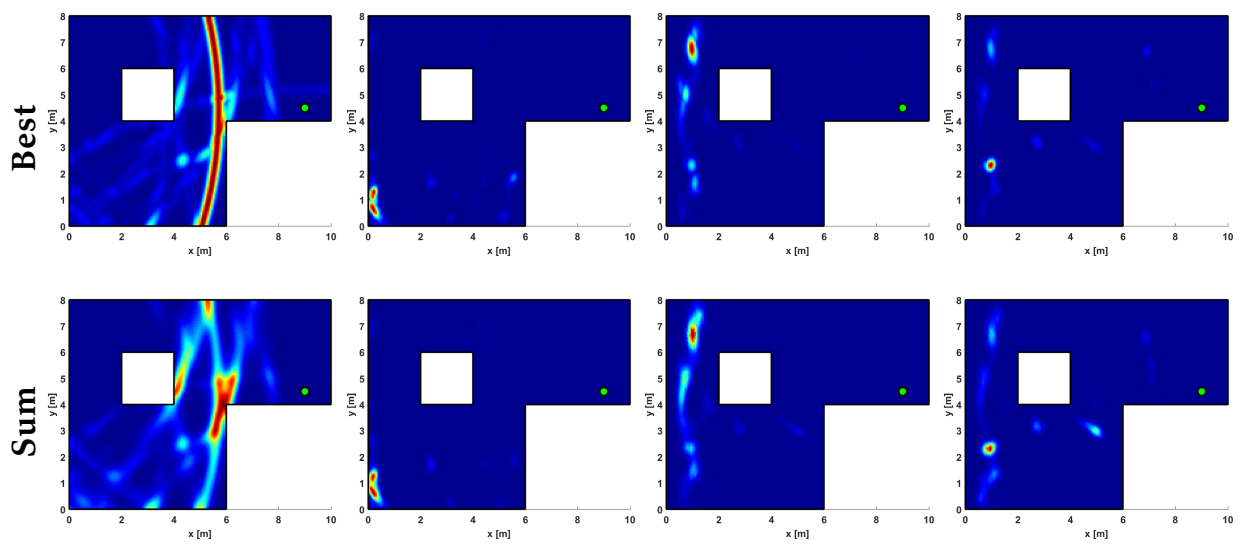

Figure 5.5: Same as Figure 5.4 but for measurements of anchor 2.

Agent Pos 1 - III Agent Pos 1 - IV

Agent Pos 2 - III

Agent Pos 2 - IV
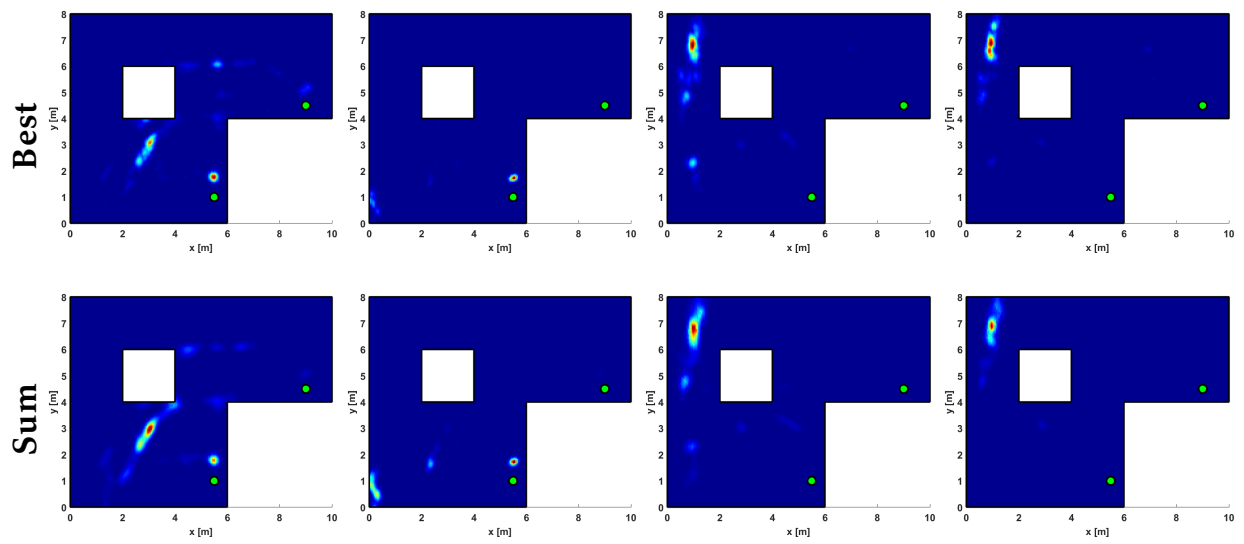

Figure 5.6: Same as Figures 5.4 and 5.5 for the joint measurements of both anchors.

How good approximation of the true distribution we get with that of the best hypothesis hence depends on the richness of the radio environment in terms of the amount of reflections and hence measurable MPCs/VAs (which in turn depends on scenario parameters such as room geometry, deployment and agent location) as well whether measurements of multiple anchors are combined or not. We limit ourselves to assuming these assumptions to be favourable and propose exactly this: For estimation models $\mathrm{C}$ through $\mathrm{F}$ in the following Sections 5.4.4 and 5.4.5 we approximate the true distribution with that of the best hypothesis. This allows us to perform the estimation without the need to calculate anything but the single best hypothesis, i.e., $B=1$, which reduces the complexity. 


\subsubsection{Results under Realistic Model Conditions}

Another relevant question considers the estimation performance of the different models of Section 5.4.2 under the realistic but problematic conditions described in Section 5.1. To recapitulate, the true association of measurements and MPCs/VAs is unknown, there are some MPCs/VAs which are not detected and there are false alarms (FAs) occurring. This is the very conditions of the generative measurement assumption IV of Section 5.4.1. Hence, we evaluate the probabilities $p_{Q \mid I V}$, as well as the resulting position estimates $\hat{\mathbf{x}}_{Q \mid I V}$ for the estimation models $Q \in\{A, B, C, D, E, F\}$. For all estimation models, except the idealized $\mathrm{A}$ and $\mathrm{B}$ for which this is not needed, we assume here the single best hypothesis of the mapping between measurements and MPCs/VAs as discussed in Section 5.4.3.

The resulting location estimates $\hat{\mathbf{x}}_{Q \mid I V}$ as well the corresponding CDF plots over the position estimation errors $\mathbf{x}-\hat{\mathbf{x}}_{Q \mid I V}$ for agent position 1 are shown in Figure 5.7. In addition, the corresponding probability distributions are shown in Figure 5.8. Noted should be that these, in contrast to those of the preceding section, show a sum over all MC realizations which effectively marginalizes away the clutter realizations whereby the probabilities for models $C$ and D - which differ only in that they take into account the clutter - are almost identical. The same goes for models $\mathrm{E}$ and $\mathrm{F}$, which thus also are almost identical. This is done in order to make the comparisons and the discussion more straightforward. The full set of results, including those of agent position 2, are provided in Section 5.A.

We make the following observations:

- The models A and B are highly idealized since the measurement to VA mapping is known. This typically gives a rather distinct maximum at the correct place. The association problem is alleviated in these models, and hence all false alarms/clutter is essentially gated away and thus provides a lower bound on the expected positioning error.

- If only the correct subset of which VAs are visible, as is the case for models $\mathrm{C}$ and D (in contrast to the ideal knowledge of models A and B), some local maxima and hence ambiguities arise in the posterior distributions and the estimation therefore becomes worse.

- If in turn, we need to form mapping hypotheses over all possible VAs, as for models $\mathrm{E}$ and $\mathrm{F}$, it becomes even more problematic with even more ambiguities and negative impact on the estimation.

- Accounting for false alarms as in estimation models D and F (in contrast to models $\mathrm{C}$ and $\mathrm{E}$ ) is beneficial since there are indeed false alarms / clutter present here.

- A richer radio environment (e.g. anchor 1) as compared to the less rich environments (e.g. anchor 2) improves the performance, which is not surprising since that gives us more and better information.

- Combining measurements from both anchors is, in most cases, beneficial. However, having poor reliability of the estimates from one anchor may some cases be worse than relying solely on the better anchor. 


\section{Estimates}
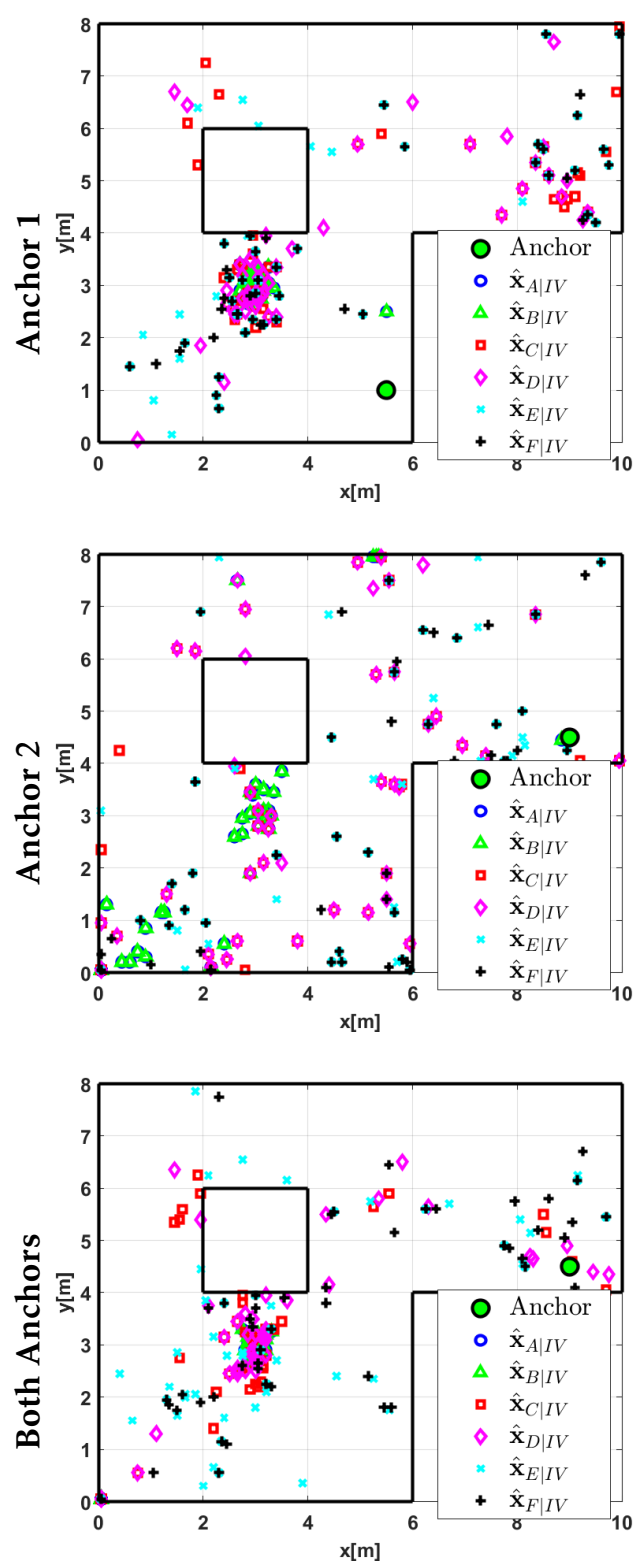

\section{Error CDFs}
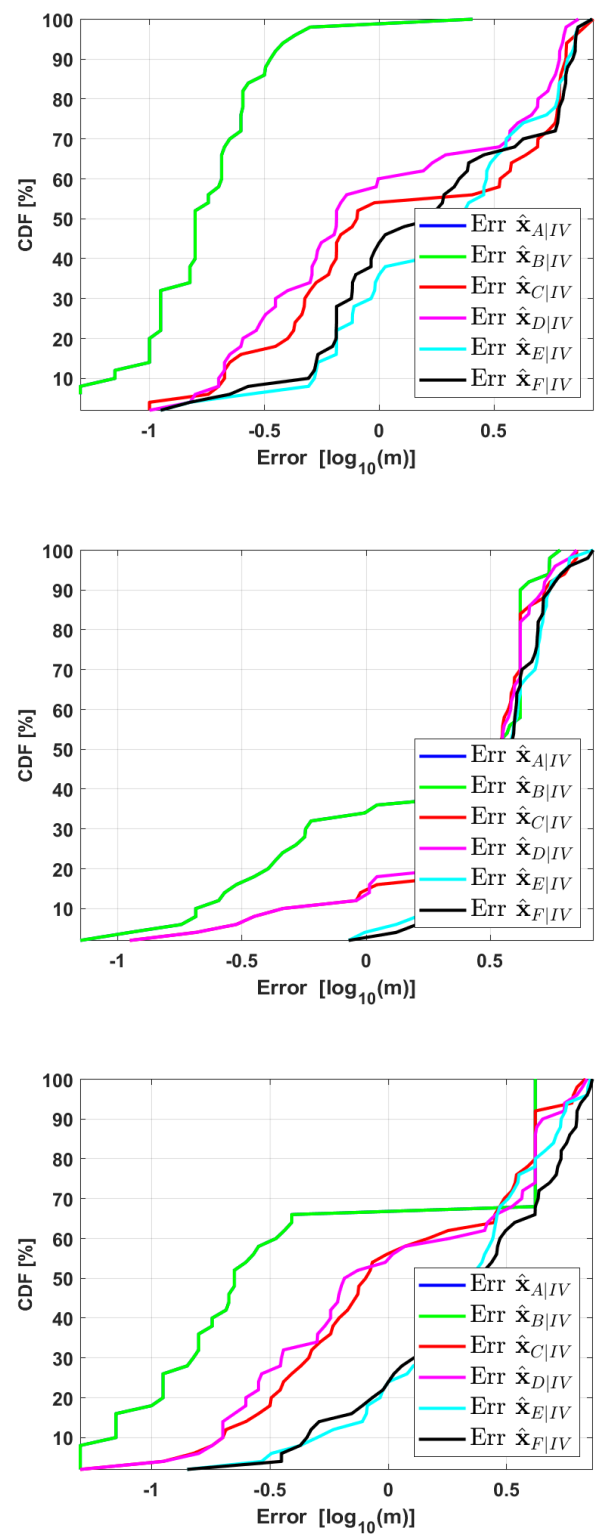

Figure 5.7: The location estimates $\hat{\mathbf{x}}_{\mathrm{Q} \mid \mathrm{IV}}$ (left column) and CDF plots of the position estimation errors $\mathbf{x}-\hat{\mathbf{x}}_{\mathrm{Q} \mid I V}$ (right column) of both anchors individually (two upper rows) and combined (bottom row) for estimation models $Q \in\{A, B, C, D, E, F\}$ and both agent positions for all MC realizations. 

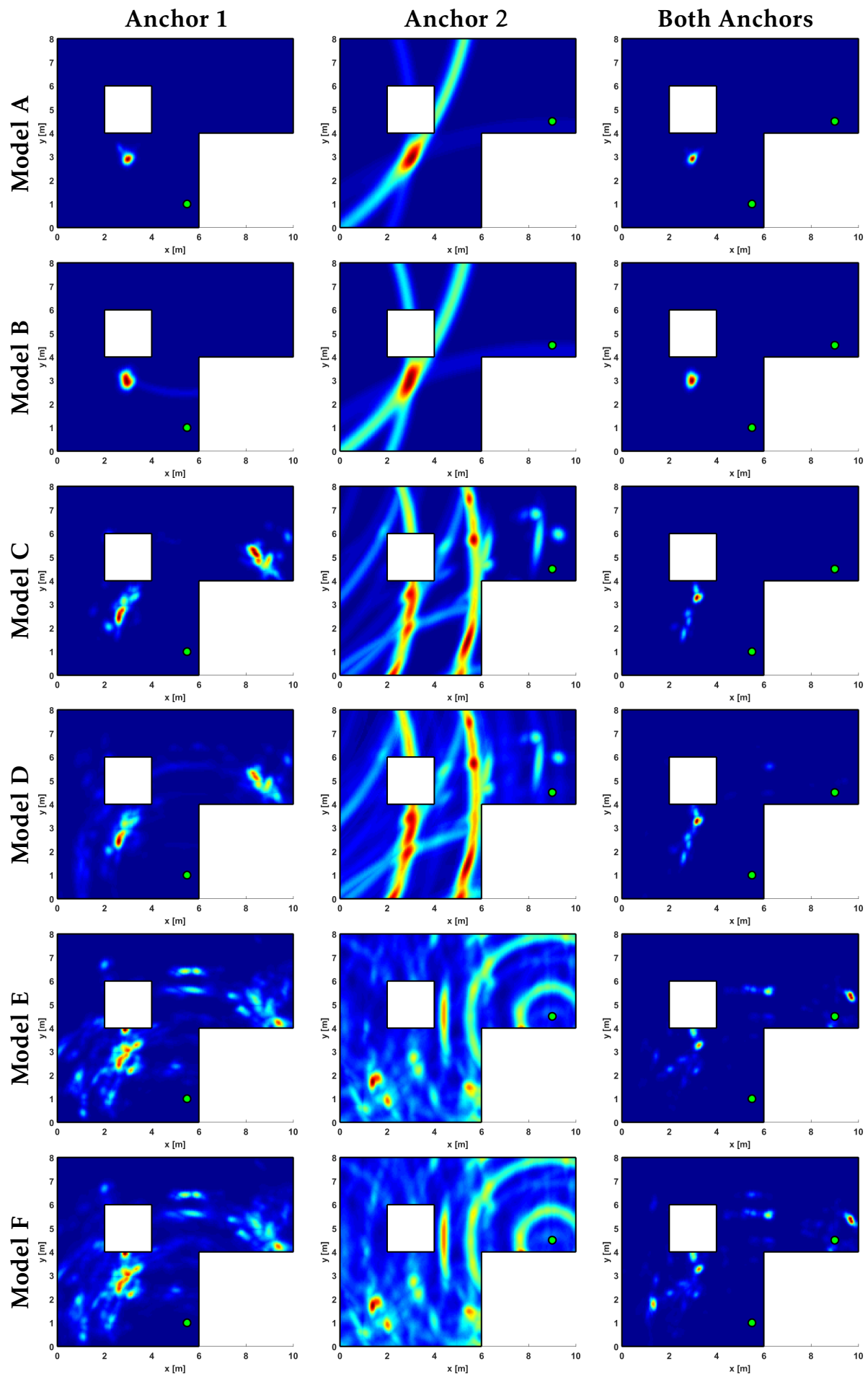

Figure 5.8: The probabilities $p_{\mathrm{Q} \mid \mathrm{IV}}$ of all estimation models when the agent is at $\mathbf{x}_{1}=[3,3]^{T}$ for the anchors individually as well as combined. 


\subsubsection{Results under Simplified Model Conditions}

Finally, we want to assess how well the various models perform under simpler conditions, wherefore we here evaluate the probabilities $p_{Q \mid R}$ as well the position estimates $\hat{\mathbf{x}}_{Q \mid R}$ for estimation models $Q \in\{A, B, C, D, E, F\}$ and measurement assumptions $R \in\{I, I I, I I, I V\}$. The combination $Q \mid I V$ was exactly what was evaluated in Section 5.4.4, but is included again for completeness. For all estimation models except A and B, we assume the single best hypothesis of the mapping between measurements and MPCs/VAs as discussed in Sections 5.3.1 and 5.4.3

The full set of results are provided in Section 5.A, whereas the resulting CDF plots over the position estimation errors $\mathbf{x}-\hat{\mathbf{x}}_{Q \mid R}$ for agent position 1 are given here in Figure 5.9. Based on these results, we make the following observations:

- When the measurements are ideal as for assumption I, the estimation is almost perfect. An exception is the models D and $\mathrm{F}$ where taking height for the clutter / false alarms comes at the cost of mistrusting valid measurements as being false alarms. For the agent position 1, this problem happens to be eliminated when combining the measurements from both anchors.

- For measurement assumptions II and III, again the negative impact of assuming false alarms when there are no such present for models D and $\mathrm{F}$ as compared to $\mathrm{C}$ and $\mathrm{E}$ as discussed in the previous bullet is clearly seen. Accounting for false alarms is thus beneficial only when such false alarms are actually present.

- Having perfect/ideal mapping knowledge (i.e., models A and B) gives better performance than if only knowing the subset of visible VAs and having to create hypotheses over these (i.e. models $\mathrm{C}$ and $\mathrm{D}$ ) but is still better than having to form hypotheses over all possible VAs (i.e., models E and F).

- The more difficult measurement assumptions, the worse the performance gets, just as would be expected. For example, estimation under the realistic measurement assumption IV is harder than under the ideal conditions of assumption I, regardless of estimation model.

The corresponding probability plots in Section 5.A, i.e., Figures 5.10 through 5.12 , confirms these observations. In addition, we also note that:

- As the measurement assumptions becomes more difficult - when going from the ideal I through the noisy II further to the non-ideal detections of III and end up which the cluttered IV - the probability distributions become more spread and ambiguous. Multiple local maxima arise, which causes problems in the estimation process.

- As the estimation models become more and more complex - when going from the ideal models A and B through when having to form mapping hypotheses over all the visible VAs in C and D and finally to when having to consider all possible VAs in $\mathrm{E}$ and $\mathrm{F}$ - again the probability distributions become more ambiguous with multiple maxima. 

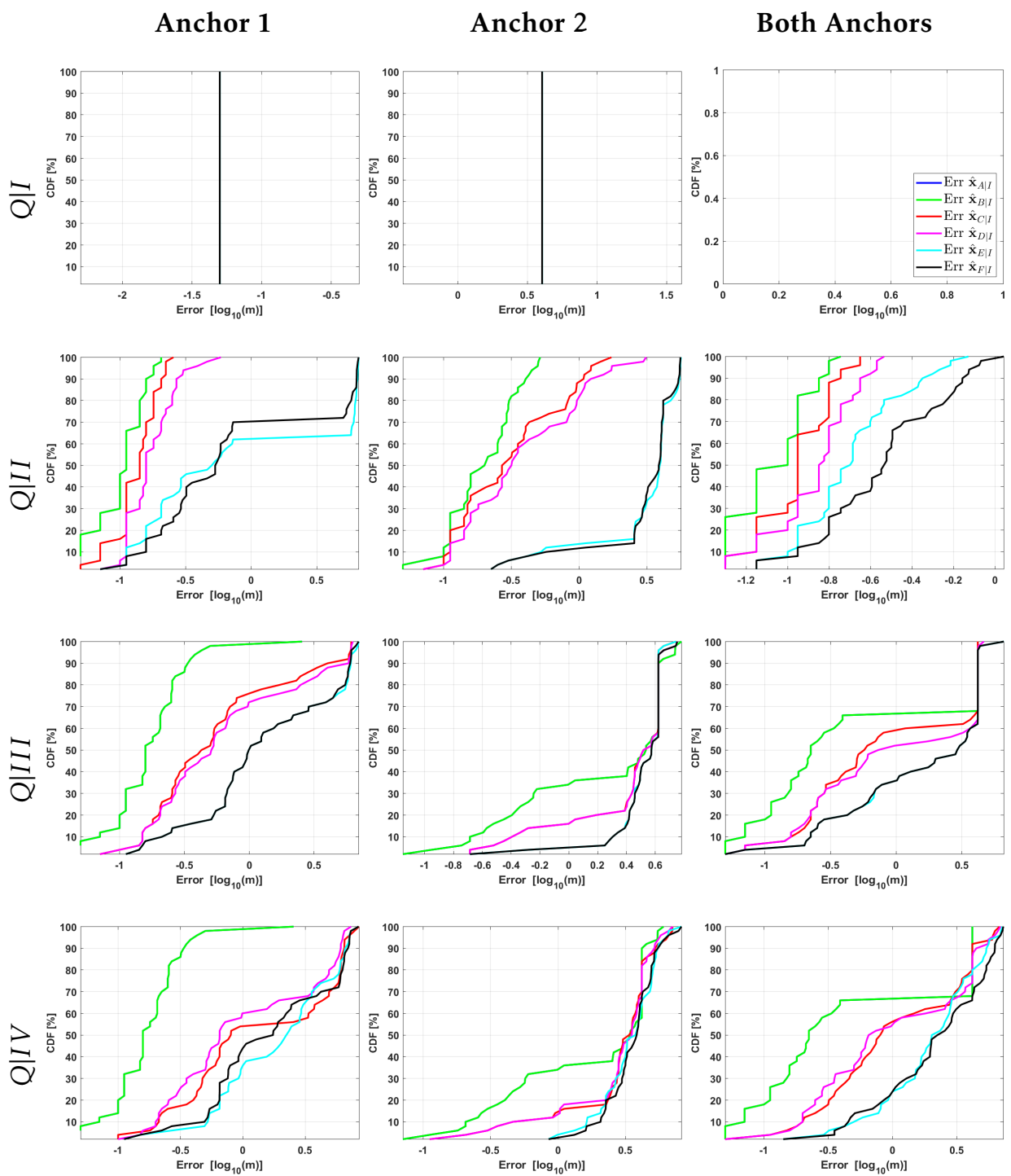

Figure 5.9: CDF plots of the position estimation errors $\mathbf{x}-\hat{\mathbf{x}}_{Q \mid R}$ of both anchors individually (two leftmost columns) and combined (rightmost column) for estimation models $Q \in\{A, B, C, D, E, F\}$ under the various measurements assumptions $R \in\{I, I I, I I, I V\}$ when the agent is at $\mathbf{x}_{1}=[3,3]^{T}$. 


\subsection{Summary}

In this chapter we have discussed the association of the measurements to the respective multi-path components of the radio channel. We have formulated the problem considering both that valid measurements may be missed and hence not detected, as well as that there may be false alarms present in the data. We have formulated the resulting probability models in a multi-target tracking inspired generic framework as a classical assignment problem, which is thereafter solved by means of a global nearest neighbour approach.

From simulations performed in an indoor scenario, we have seen that:

- The single best association hypothesis is generally a good approximation that avoids the calculation of a vast number of possible hypotheses.

- The richer radio environment there is in terms of many reflections / MPCs, the better positioning performance can be expected, since this increases the amount of information. At the same time, this causes the association problem size to grow larger.

- Prior knowledge of the potential MPCs that needs to be hypothesised over, significantly reduces the problem complexity.

- Accounting for erroneous measurements such as false alarms and missed detections is crucial if such are indeed present in the actual measurements. If not, there is a cost of accounting for such behaviour.

- Combining measurements from multiple anchors is beneficial, since also this increases the amount of information available in the estimation process.

It should be pointed out that we in this work have only utilized the pseudorange measurements and not the received signal strength or channel amplitudes of the respective MPC. This information could possibly also be included, which would essentially add one dimension to the hypotheses and hence render a more complex problem but, most likely, also give even better localization accuracy because of the increased amount of available information. This could however be problematic since the channel amplitudes are typically less stable and more prone to disturbances than the pseudo-range measurements. This topic is left for further studies. 


\section{Appendix}

\section{A Additional Figures}

This appendix contains additional plots from the numeric evaluations performed in this chapter.

First, the sum of the probabilities $p_{Q \mid R}$ for all $50 \mathrm{MC}$ realizations for all models plotted over a grid of the entire room when the agent is at $\mathbf{x}_{1}=[3,3]^{T}$ is shown in Figures $5.10-5.12$, whereas when the agent is at $\mathbf{x}_{2}=[1,7]^{T}$ in Figures $5.13-5.15$. Since this presents a sum over all MC realizations, which effectively marginalizes away the clutter realizations, the probabilities for estimation models C and D - which differ only in that they take into account the clutter - are almost identical. The same goes for estimation models $\mathrm{E}$ and $\mathrm{F}$, which are thus also almost identical.

Secondly, in Figures 5.16 and 5.17 the resulting CDFs of the localization estimation errors $\mathbf{x}-\hat{\mathbf{x}}_{Q \mid R}$ are shown for all the different models and agent positions.

Finally, we show in Figure 5.18 scatter plots of the actual location estimates for the most realistic/complex measurement assumption IV, i.e., $\hat{\mathbf{x}}_{\mathrm{Q} \mid I V}$. 

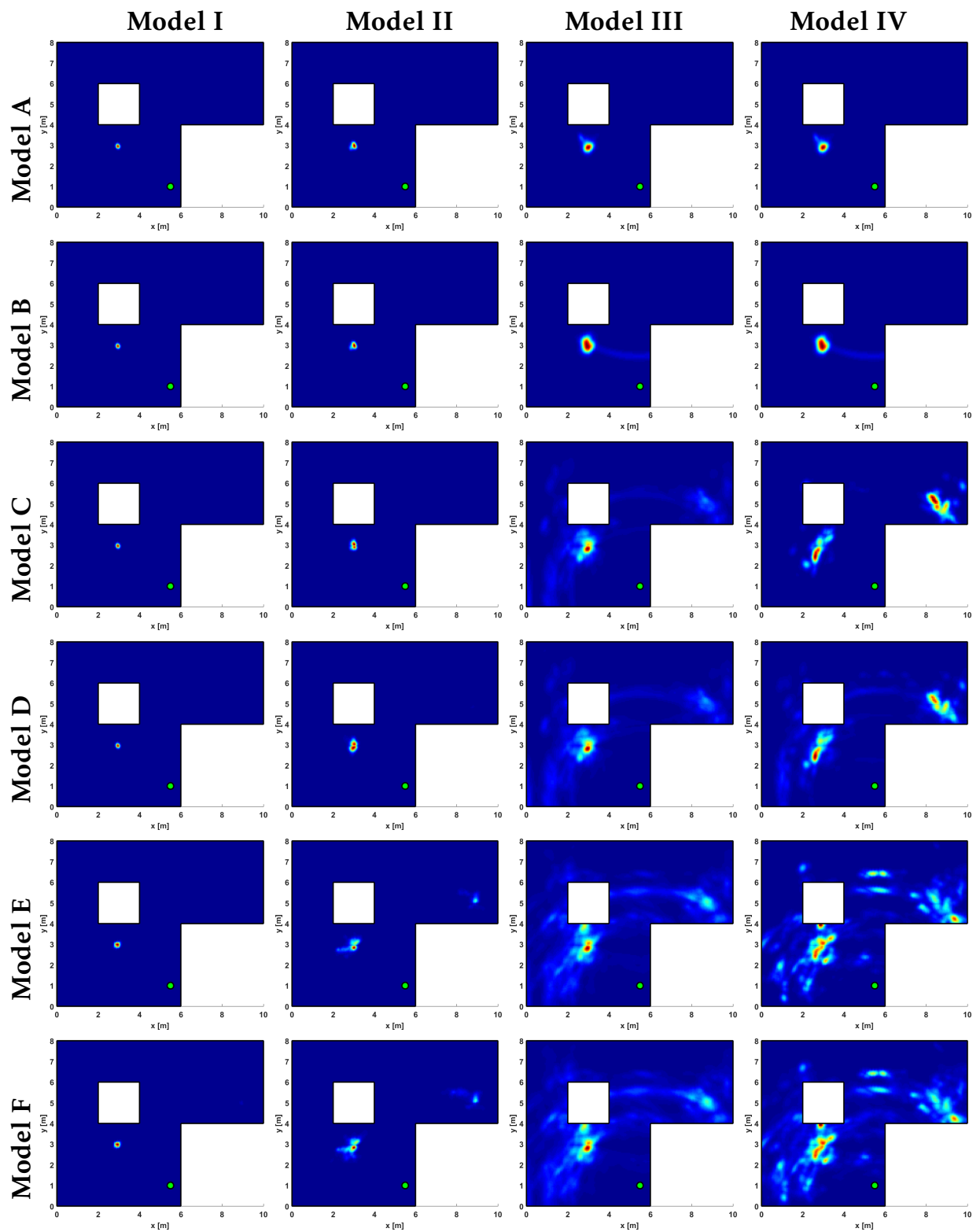

Figure 5.10: The probabilities $p_{Q \mid R}$ of all estimation models and measurement assumptions when the agent is at $\mathbf{x}_{1}=[3,3]^{T}$ for anchor 1 . 

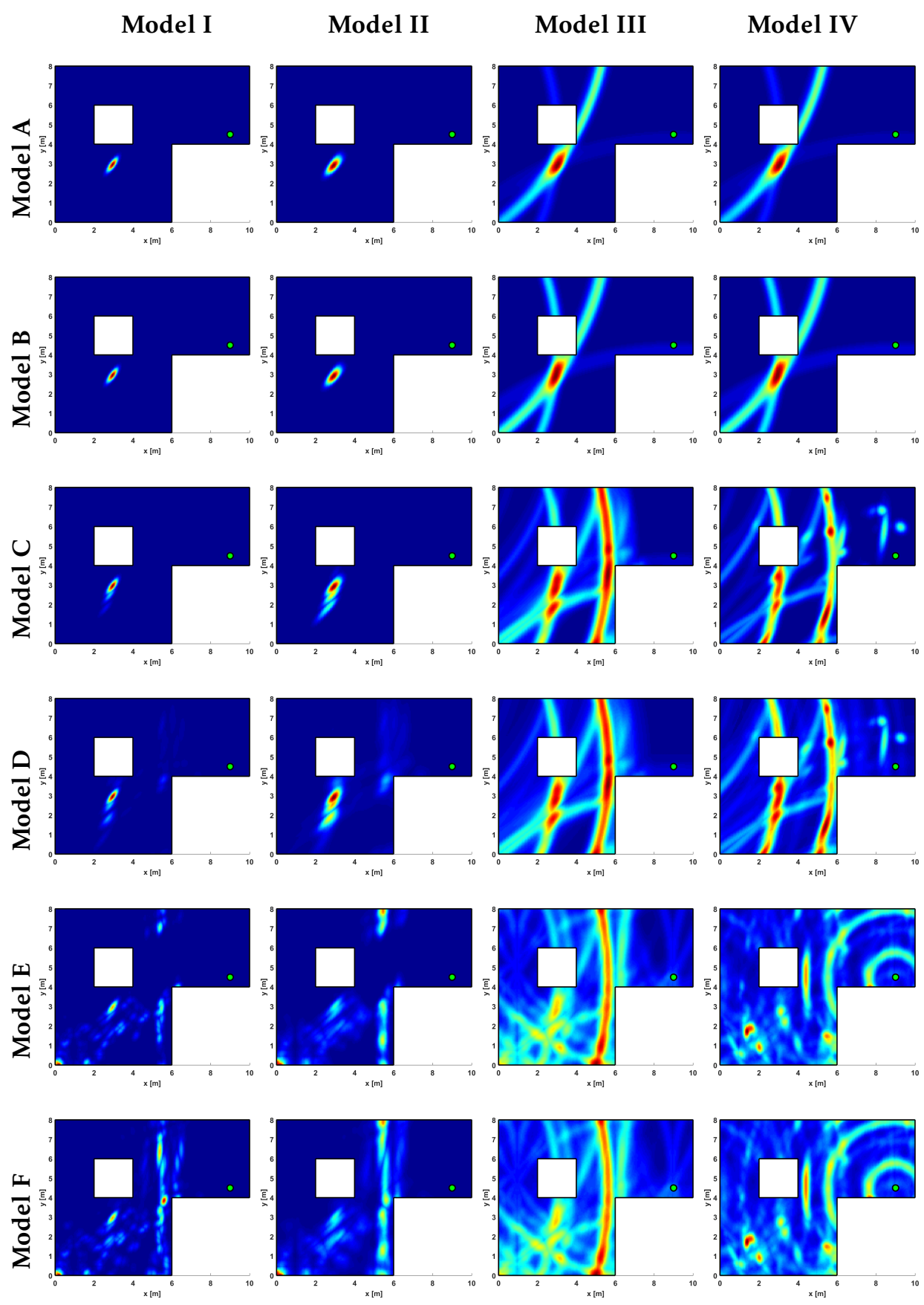

Figure 5.11: Same as Figure 5.10 but for anchor 2. 

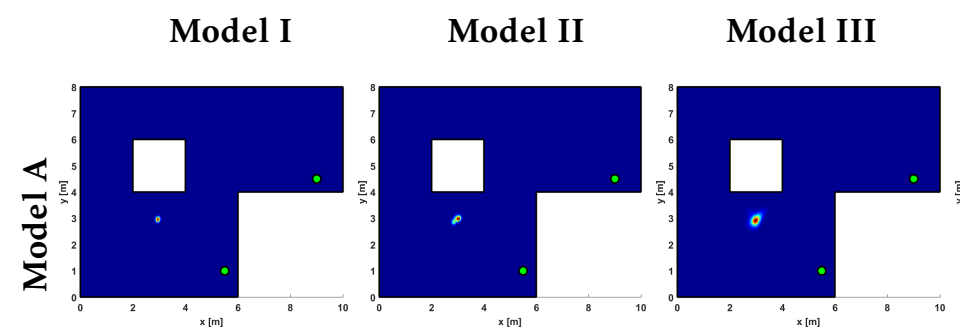

Model IV
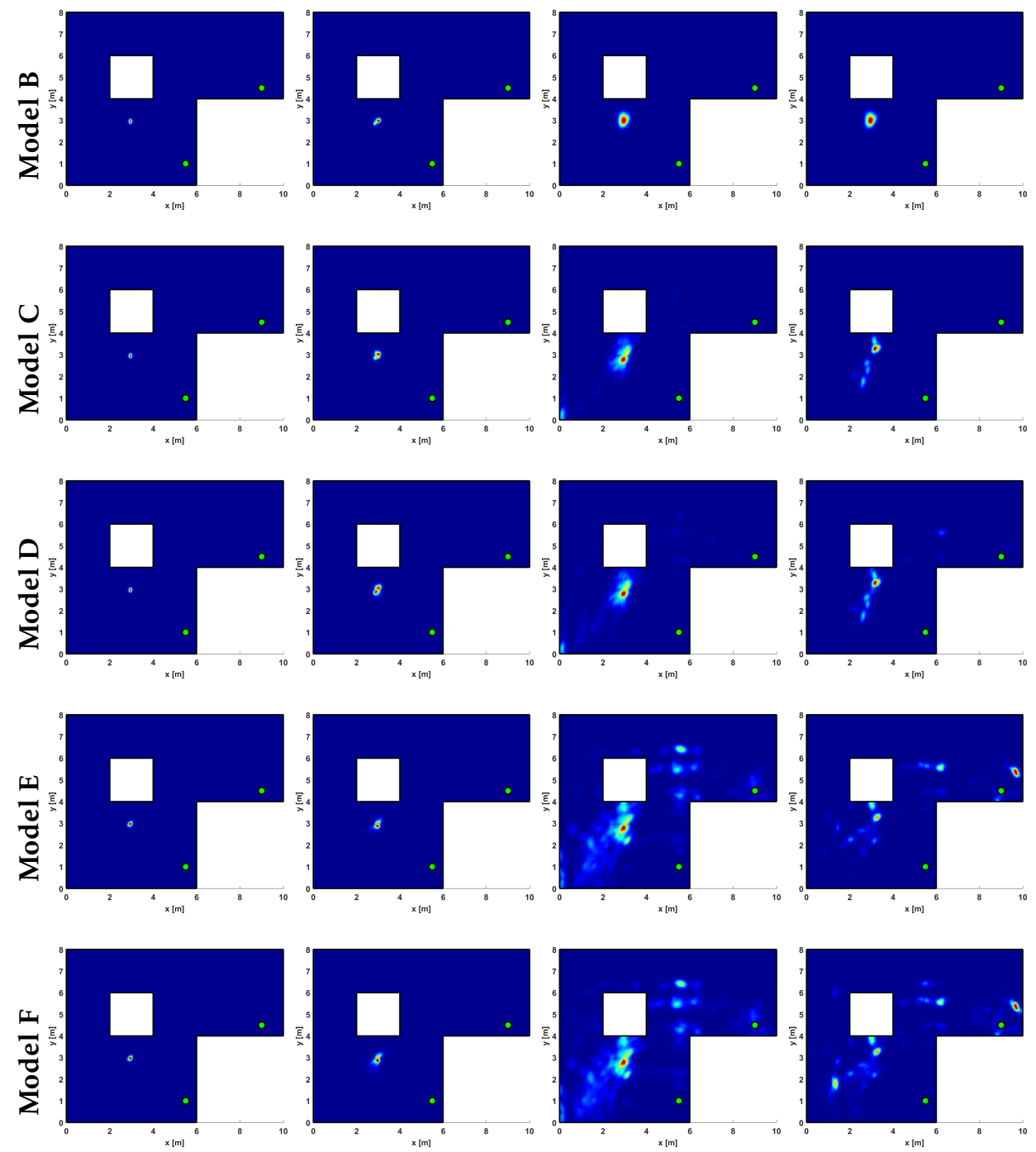

Figure 5.12: Same as Figures 5.10 and 5.11 but for both anchors combined. 

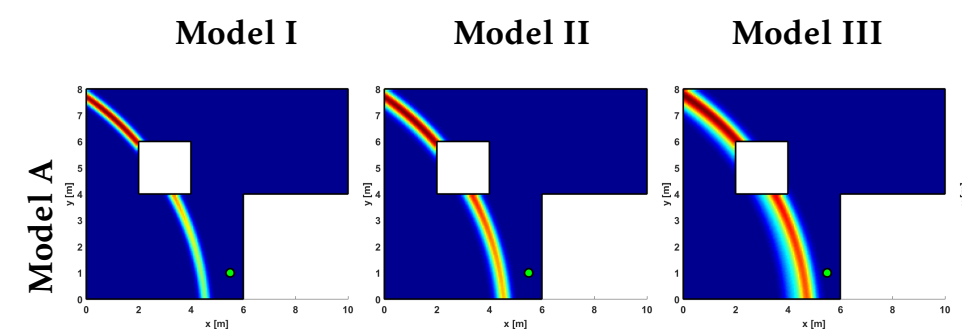

Model IV
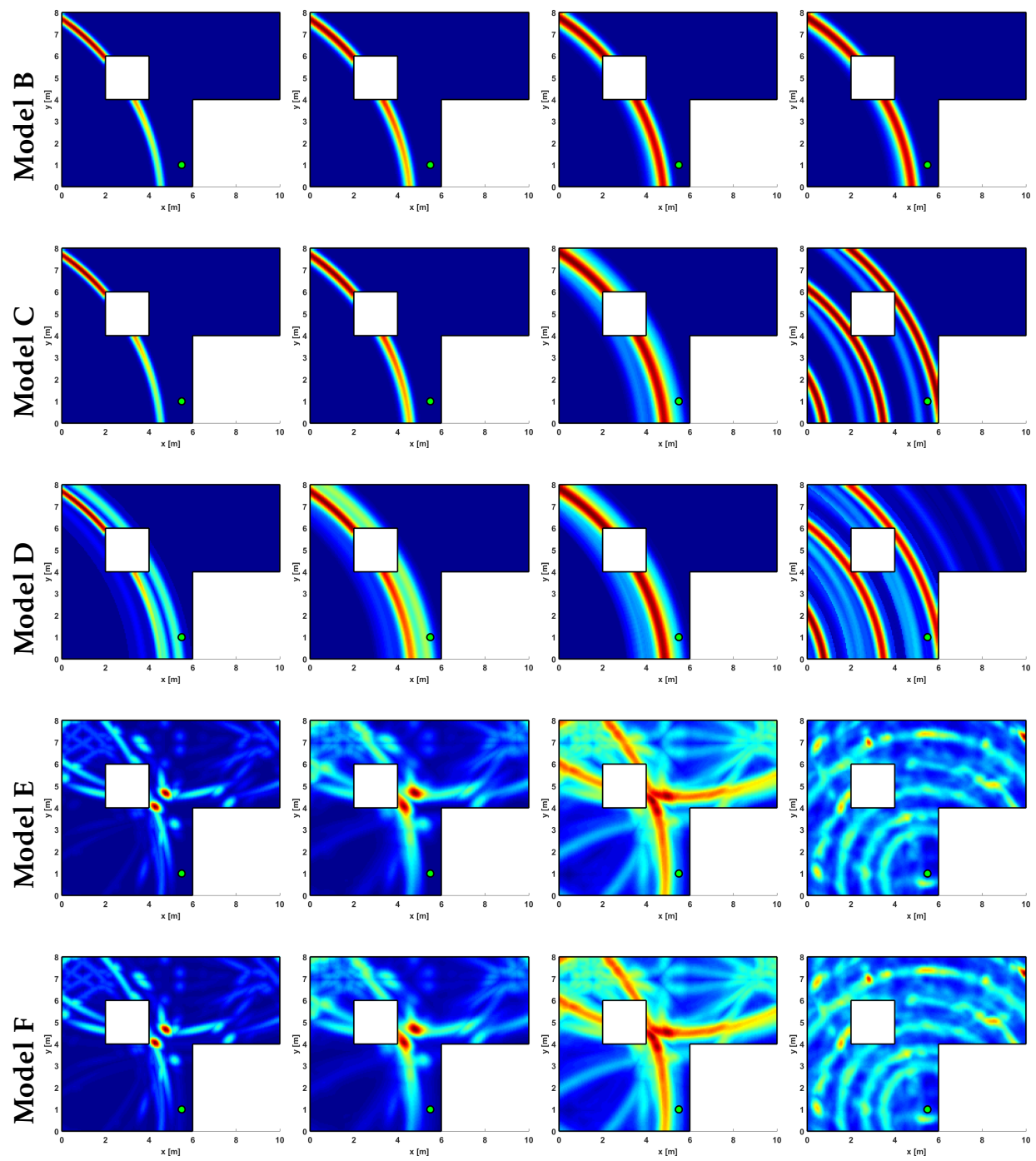

Figure 5.13: The probabilities $p_{Q \mid R}$ of all estimation models and measurement assumptions when the agent is at $\mathbf{x}_{2}=[1,7]^{T}$ for anchor 1 . 

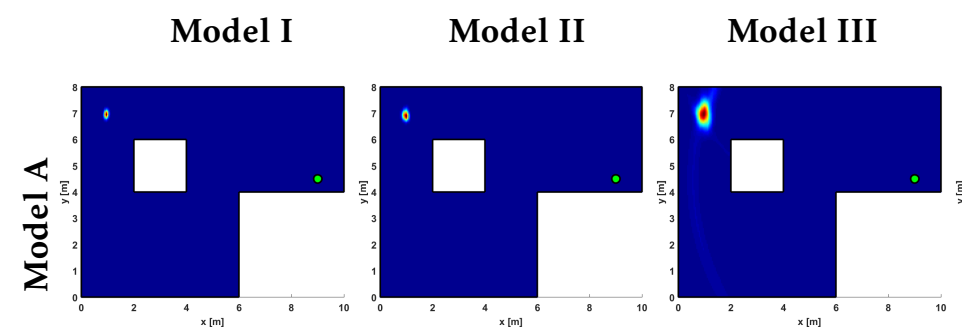

Model IV
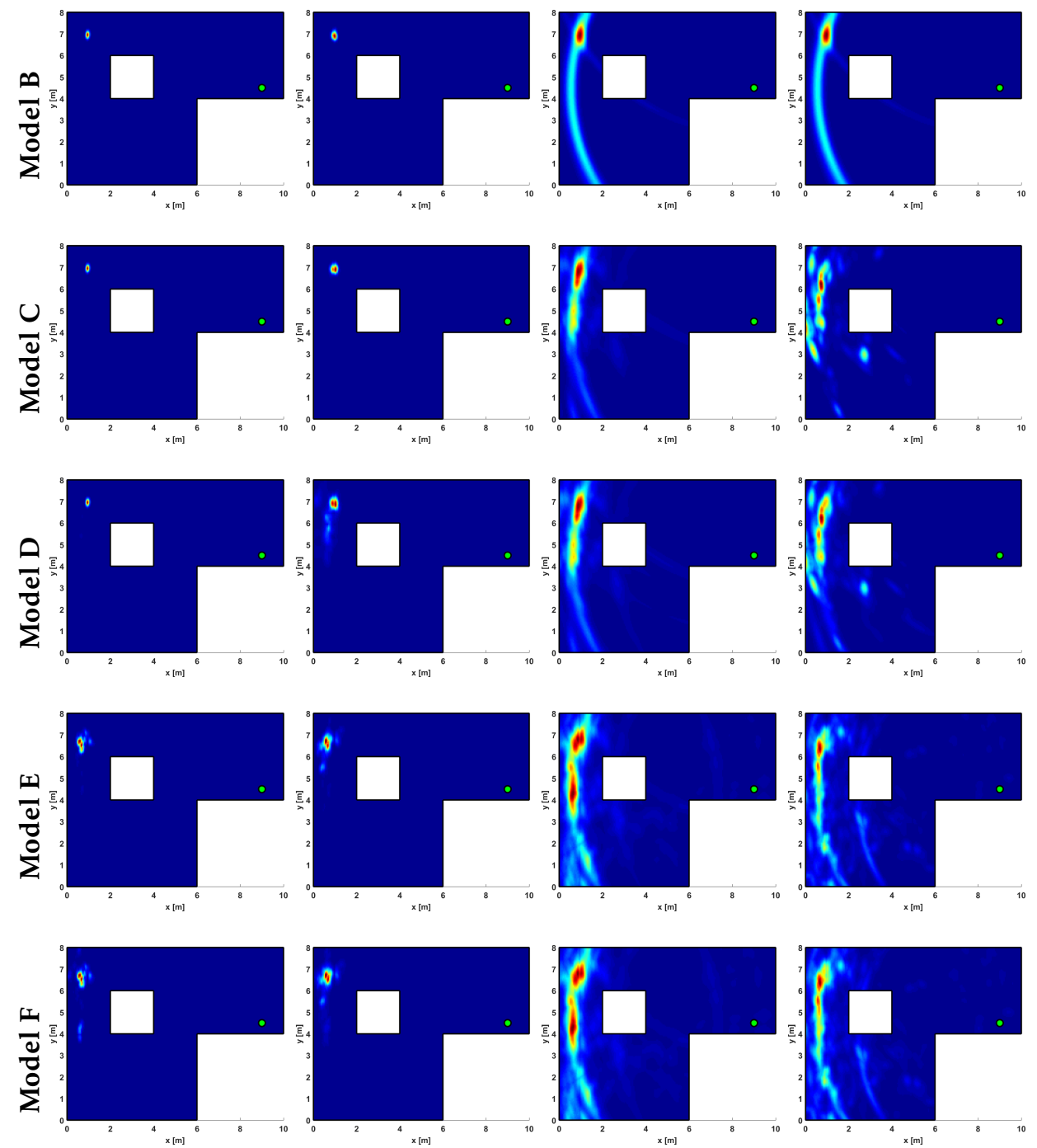

Figure 5.14: Same as Figure 5.13 but for anchor 2. 

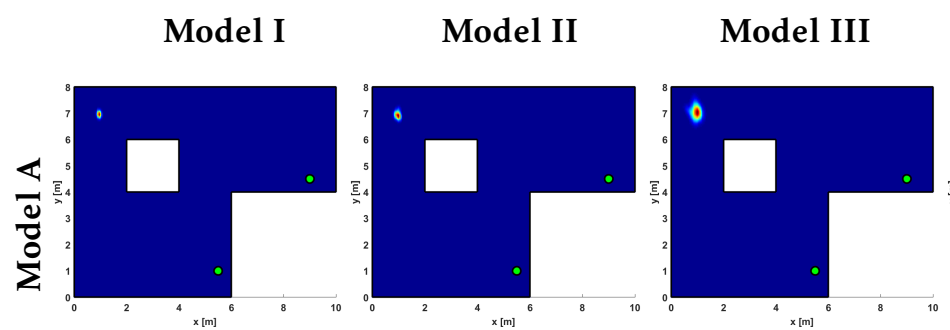

Model IV
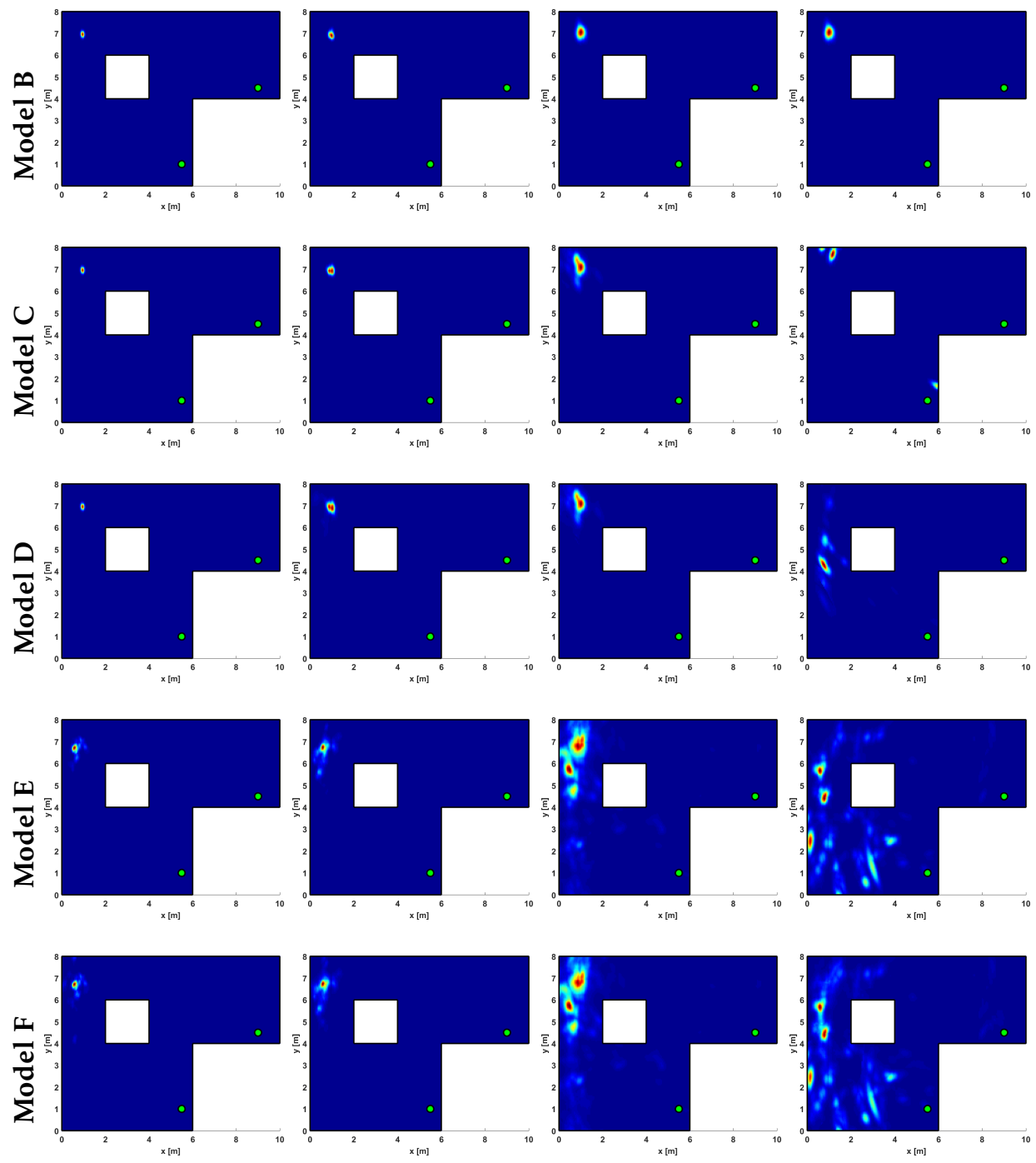

Figure 5.15: Same as Figures 5.13 and 5.14 but for both anchors combined. 
Anchor 1
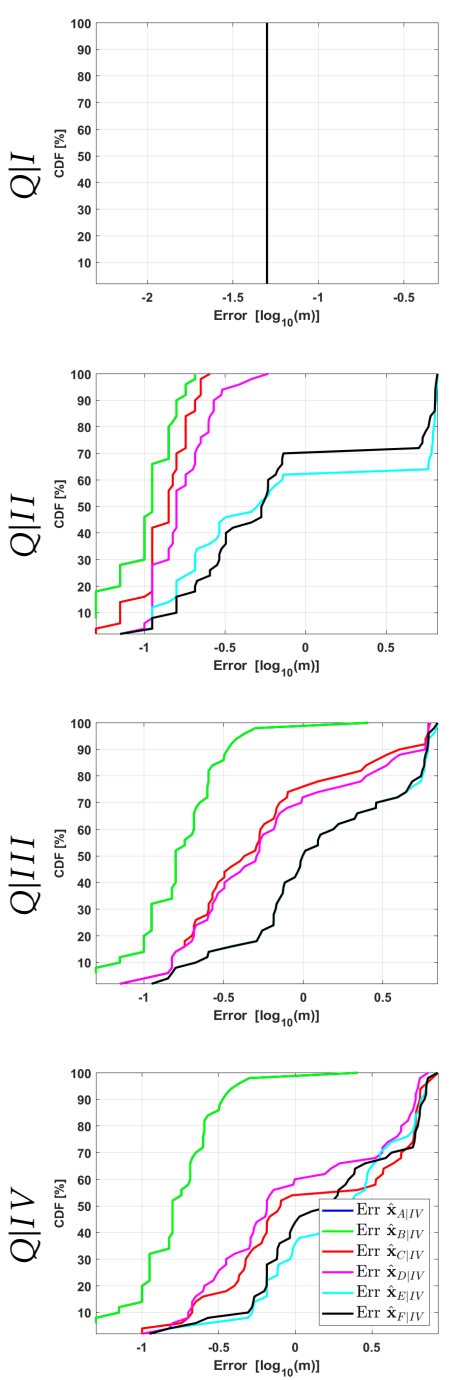

Anchor 2
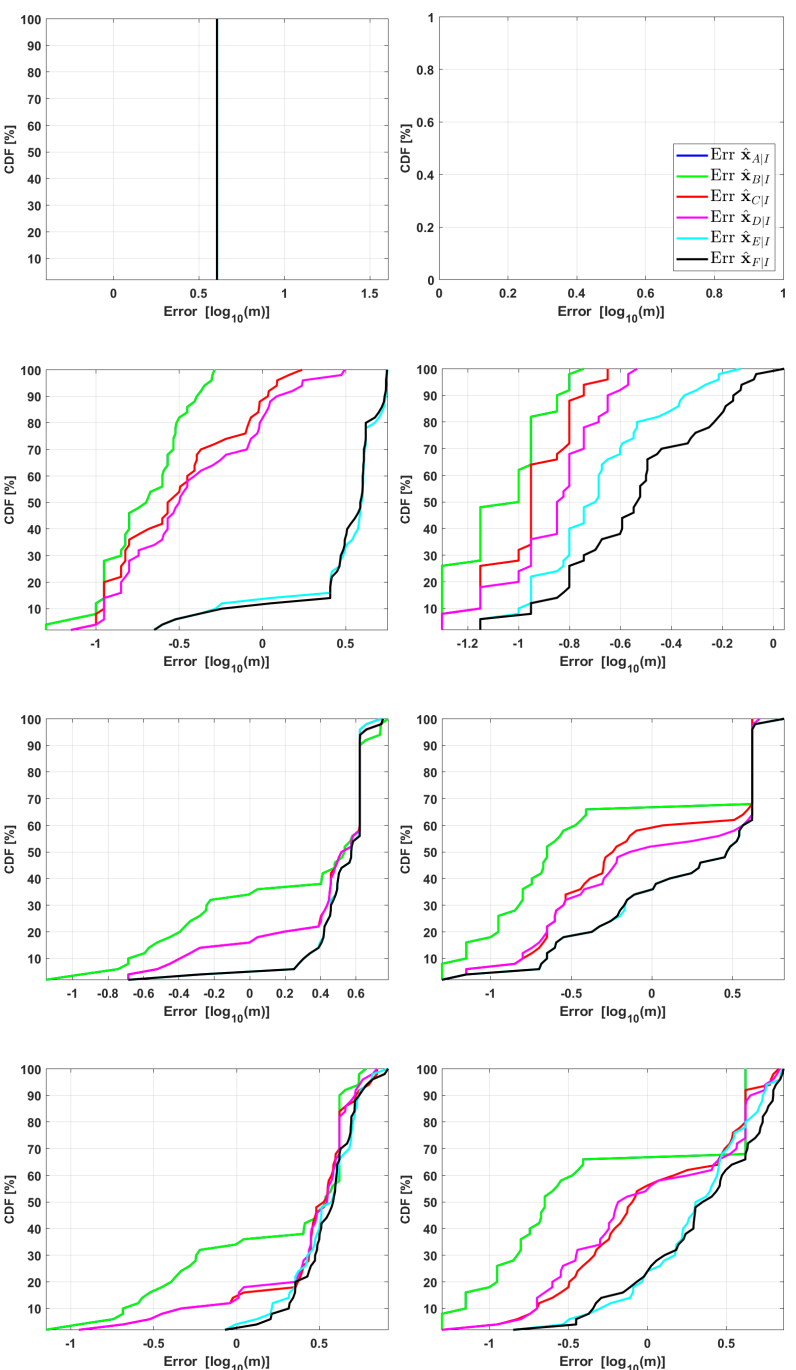

Both Anchors
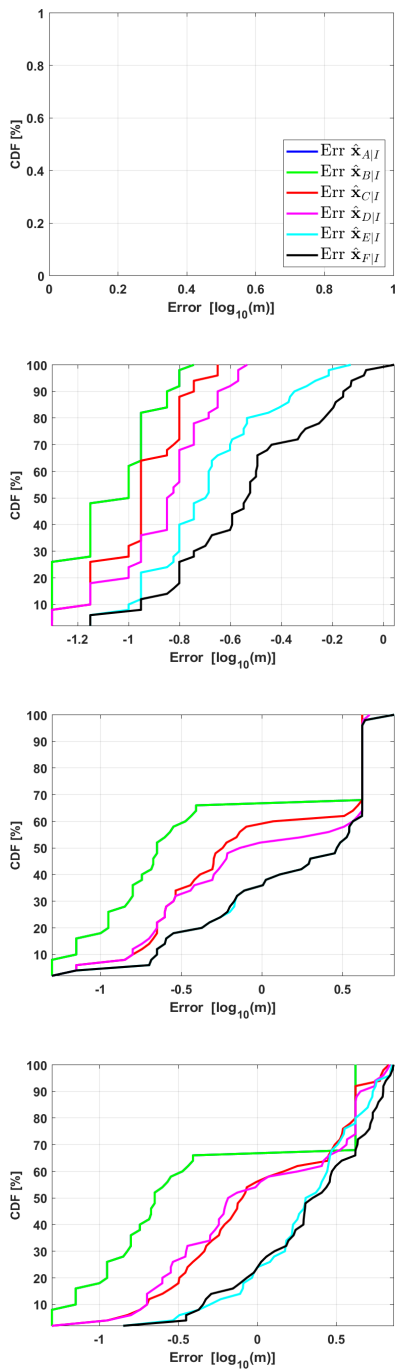

Figure 5.16: CDF plots of the localization estimation errors $\mathbf{x}-\hat{\mathbf{x}}_{Q \mid R}$ of both anchors individually (two leftmost columns) and combined (rightmost column) for estimation models $Q \in\{A, B, C, D, E, F\}$ under the various measurements assumptions $R \in\{I, I I, I I, I V\}$ when the agent is at $\mathbf{x}_{1}=[3,3]^{T}$. 

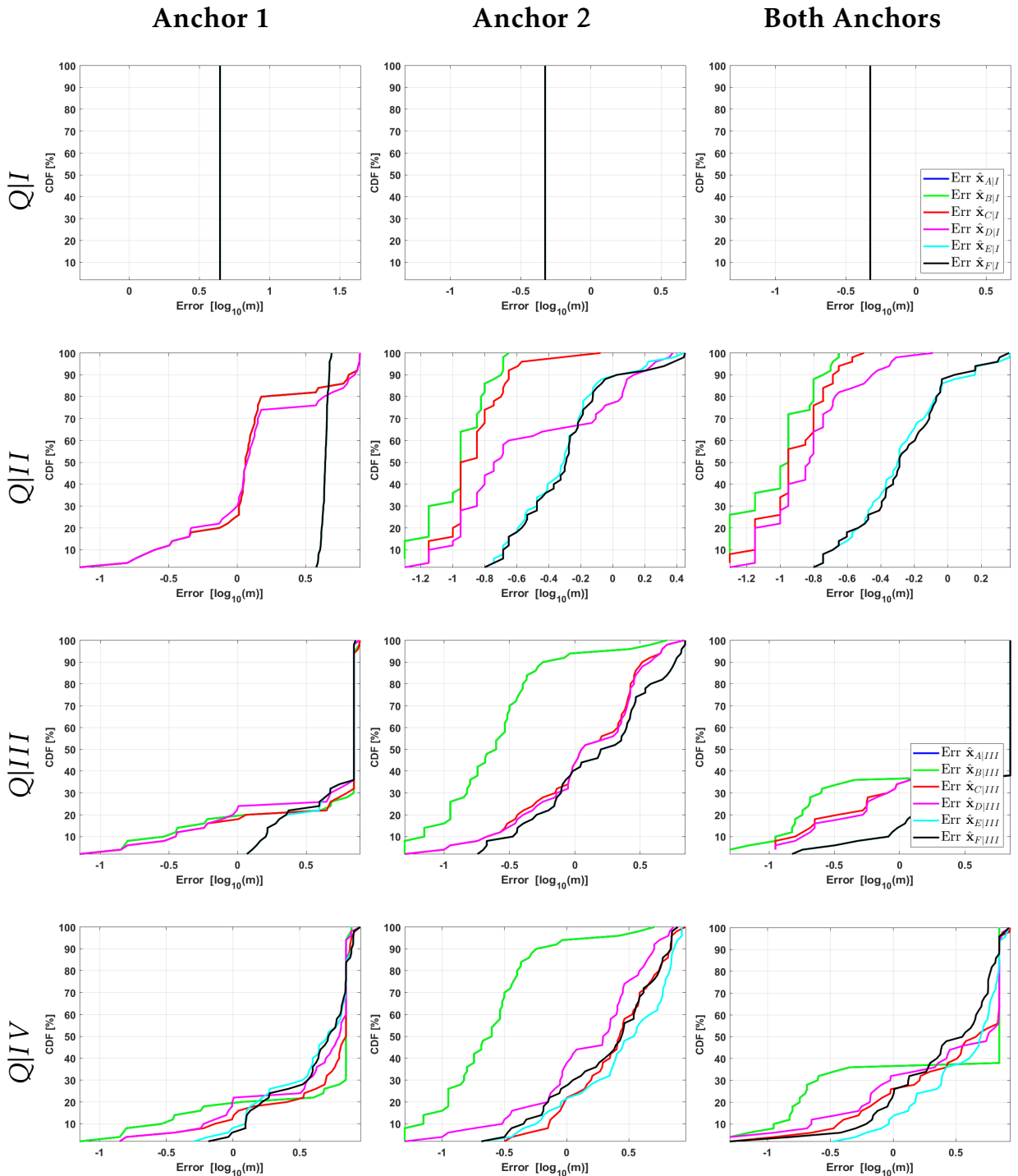

Figure 5.17: CDF plots of the localization estimation errors $\mathbf{x}-\hat{\mathbf{x}}_{Q \mid R}$ of both anchors individually (two leftmost columns) and combined (rightmost column) for estimation models $Q \in\{A, B, C, D, E, F\}$ under the various measurement assumptions $R \in\{I, I I, I I, I V\}$ when the agent is at $\mathbf{x}_{2}=[1,7]^{T}$. 
Agent Position $\mathbf{x}_{1}=[3,3]^{T}$
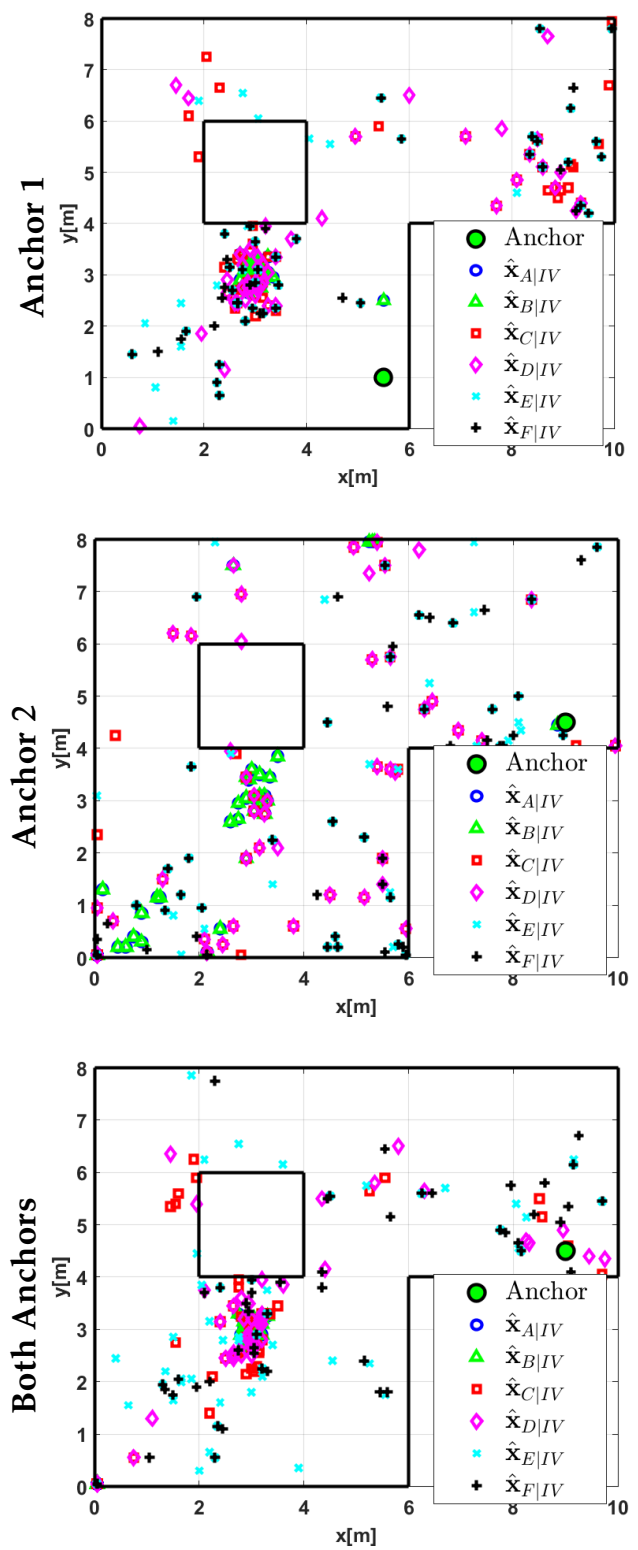

Agent Position $\mathbf{x}_{2}=[1,7]^{T}$
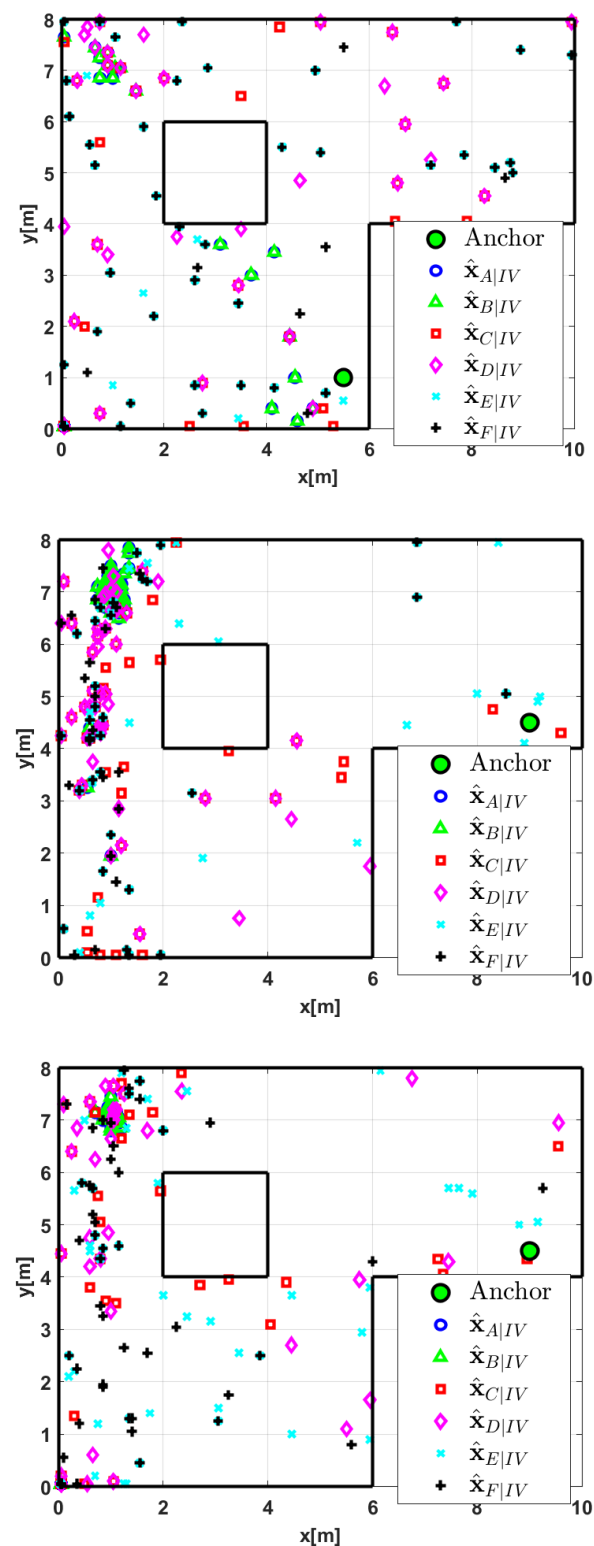

Figure 5.18: The resulting localization estimates $\hat{\mathbf{x}}_{Q \mid I V}$ of both anchors individually (two upper rows) and combined (bottom row) for estimation models $Q \in\{A, B, C, D, E, F\}$ and both agent positions for all MC realizations. 



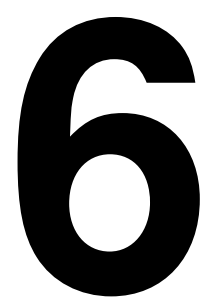

\section{Performance Bounds}

In this chapter we investigate the theoretical localization performance bounds achievable when utilizing all, or parts of, the multipath-related information of the radio channel.

This chapter is organized as follows: In Section 6.1 we introduce the Positioning Error Bound (PEB) which is the metric used in the subsequent evaluations, as well as the impact of certain modelling choices on the localization performance. We also investigate the impact of having access to measurements of only a subset of the available MPCs as well as those of multiple anchors and/or in both the uplink and downlink directions. Numerical results of these various aspects are presented in Section 6.2, after which Section 6.3 summarizes the results.

\subsection{Positioning Error Bound}

From [45], [20] and [16] we know that for a parameter vector $\theta$, the covariance matrix of any unbiased estimator $\hat{\theta}$ is bounded by the Cramér-Rao Lower Bound (CRLB) as

$$
\operatorname{Cov}(\hat{\boldsymbol{\theta}})=\mathbb{E}\left\{(\boldsymbol{\theta}-\hat{\boldsymbol{\theta}})(\boldsymbol{\theta}-\hat{\boldsymbol{\theta}})^{H}\right\} \geq \mathbf{J}_{\boldsymbol{\theta}}^{-1}
$$

where $\mathbf{J}_{\theta}$ is the Fisher Information Matrix (FIM) of the vector $\boldsymbol{\theta}$. Given an alternative parameter vector $\psi$, which has the FIM $\mathbf{J}_{\psi}$, we can as discussed in [48] relate these two by the Jacobian transformation matrix $\mathbf{T}=\frac{\partial \psi}{\partial \theta}$ as

$$
\mathbf{J}_{\theta}=\mathbf{T} \mathbf{J}_{\psi} \mathbf{T}^{T}
$$

In our case, following closely what was done in e.g. [29], we start by defining the parameter vector $\theta=\left[\begin{array}{lll}\mathbf{x}^{T} & \mathfrak{k} \mathfrak{v}(\alpha)^{T} & \operatorname{Im}(\alpha)^{T}\end{array}\right]^{T}$ where (as before) $\mathbf{x} \in \mathbb{R}^{2}$ is the position of the agent, $\alpha$ constitutes the stacked values of the MPC amplitudes 
$\left\{\alpha_{m}^{(k)}\right\}$ (which are treated as nuisance parameters here) for all anchors $m \in \mathcal{M}$ and the related MPCs $k \in \mathcal{K}_{m}$. Here we have defined $\mathcal{M}$ as the set of observed anchors and $\mathcal{K}_{m}$ as the set of observed MPCs with respect to the specific anchor $m$. Further, $\operatorname{Re}(\cdot)$ and $\operatorname{Im}(\cdot)$ denote the real and imaginary parts, respectively. Hence, the total number of elements in $\alpha$ is $K=\sum_{m \in \mathcal{M}}\left|\mathcal{K}_{m}\right|$ so that $\alpha \in \mathbb{R}^{K}$ and thus $\theta \in$ $\mathbb{R}^{2+2 K}$. We further let $\tau$ constitute the stacked values of the MPC delays $\left\{\tau_{m}^{(k)}\right\}$ (which also contains $K$ elements) and define the alternative parameter vector $\psi=$ $\left[\begin{array}{lll}\boldsymbol{\tau}^{T} & \operatorname{Re}(\alpha)^{T} & \operatorname{Im}(\alpha)^{T}\end{array}\right]^{T} \in \mathbb{R}^{3 K}$. The Jacobian matrix $\mathbf{T}$ in (6.2) now becomes

$$
\mathbf{T}=\frac{\partial \psi}{\partial \boldsymbol{\theta}}=\left[\begin{array}{cc}
\mathbf{H}_{2 \times K} & \mathbf{0}_{2 \times 2 K} \\
\mathbf{0}_{2 K \times K} & \mathbf{I}_{2 K \times 2 K}
\end{array}\right]
$$

where

$$
\mathbf{H}=\frac{1}{c}\left[\begin{array}{ccc}
\cos \phi^{(1)} & \cdots & \cos \phi^{(K)} \\
\sin \phi^{(1)} & \cdots & \cos \phi^{(K)}
\end{array}\right],
$$

so that its $k$ th column is given by $\frac{1}{c}\left[\cos \phi^{(k)} \sin \phi^{(k)}\right]^{T}$. Here $\phi^{(k)}$ is the DL angleof-arrival for MPC $k_{m}$ received by the agent at $\mathbf{x}$, which thus is the same as the angle from the $k_{m}$ th VA at $\left\{\mathbf{p}_{m}^{(k)}\right\}$ to the agent:

$$
\phi_{m}^{(k)}=\tan ^{-1}\left(\frac{y-p_{y, m}^{(k)}}{x-p_{x, m}^{(k)}}\right) .
$$

In this chapter we do not present the full details on how to calculate the FIM $\mathbf{J}_{\psi}$ in $(6.2)$, but instead refer the interested reader to $[29,48]$. The result of these calculations is however that

$$
\mathbf{J}_{\psi}=\left[\begin{array}{ccc}
\boldsymbol{\Lambda}_{\mathrm{A}} & \boldsymbol{\Lambda}_{\mathrm{B}}^{\mathrm{R}} & \boldsymbol{\Lambda}_{\mathrm{B}}^{\mathrm{I}} \\
\left(\boldsymbol{\Lambda}_{\mathrm{B}}^{\mathrm{R}}\right)^{T} & \boldsymbol{\Lambda}_{\mathrm{C}}^{\prime} & \mathbf{0} \\
\left(\boldsymbol{\Lambda}_{\mathrm{B}}^{\mathrm{I}}\right)^{T} & \mathbf{0} & \boldsymbol{\Lambda}_{\mathrm{C}}^{\prime}
\end{array}\right]=\left[\begin{array}{cc}
\boldsymbol{\Lambda}_{\mathrm{A}} & \boldsymbol{\Lambda}_{\mathrm{B}} \\
\boldsymbol{\Lambda}_{\mathrm{B}}^{T} & \boldsymbol{\Lambda}_{\mathrm{C}}
\end{array}\right]
$$

where the $(i, j)$ th element of the matrices $\Lambda_{\mathrm{A}}, \Lambda_{\mathrm{B}}^{\mathrm{R}}, \boldsymbol{\Lambda}_{\mathrm{B}}^{\mathrm{I}}$ and $\boldsymbol{\Lambda}_{\mathrm{C}}^{\prime}$ are given by

$$
\begin{aligned}
& {\left[\boldsymbol{\Lambda}_{\mathrm{A}}\right]_{i, j}=-\frac{2}{N_{0}} \operatorname{ke}\left\{[\alpha]_{i}[\alpha]_{j}\right\}[w]_{i}[w]_{j} \frac{\partial^{2}}{\partial \tau^{2}} R_{s}\left([\boldsymbol{\tau}]_{i}-[\boldsymbol{\tau}]_{j}\right)} \\
& {\left[\boldsymbol{\Lambda}_{\mathrm{B}}^{\mathrm{R}}\right]_{i, j}=\frac{2}{N_{0}} \operatorname{ke}\left\{[\alpha]_{i}\right\}[w]_{i}[w]_{j} \frac{\partial}{\partial \tau} R_{s}\left([\boldsymbol{\tau}]_{i}-[\boldsymbol{\tau}]_{j}\right)} \\
& {\left[\boldsymbol{\Lambda}_{\mathrm{B}}^{\mathrm{I}}\right]_{i, j}=\frac{2}{N_{0}} \operatorname{Im}\left\{[\alpha]_{i}\right\}[w]_{i}[w]_{j} \frac{\partial}{\partial \tau} R_{s}\left([\boldsymbol{\tau}]_{i}-[\boldsymbol{\tau}]_{j}\right)} \\
& {\left[\boldsymbol{\Lambda}_{\mathrm{C}}^{\prime}\right]_{i, j}=\frac{2}{N_{0}}[\boldsymbol{w}]_{i}[w]_{j} R_{s}\left([\boldsymbol{\tau}]_{i}-[\boldsymbol{\tau}]_{j}\right)}
\end{aligned}
$$

and where $[\alpha]_{i}$ and $[\tau]_{i}$ denotes the $i$ th element of the vectors $\alpha$ and $\tau$, respectively. The auto-correlation function $R_{S}(\tau)$ was defined in (2.29), and was plotted 
together with it first and second derivatives in Figure 2.12b of Section 2.4. Further, the weight-vector $w \in \mathbb{R}^{K}$ in (6.7) is defined as

$$
[w]_{i}=\left(\frac{N_{0}}{N_{0}+T_{S} S_{v}\left([\tau]_{i}\right)}\right)^{1 / 2} .
$$

Combining (6.3) and (6.6) allows us to calculate $\mathbf{J}_{\theta}$ in (6.2), and subsequently $\mathbf{J}_{\theta}^{-1}$ in (6.1). For the latter we are however only interested the estimation accuracy of the agent's position $\mathbf{x}$, which is represented by its top-left $2 \times 2$ sub-block $\left[\mathbf{J}_{\theta}^{-1}\right]_{2 \times 2}$. By means of block-wise matrix inversion (see e.g. [40]), this can be calculated as

$$
\left[\mathbf{J}_{\boldsymbol{\theta}}^{-1}\right]_{2 \times 2}=\left(\mathbf{H} \boldsymbol{\Lambda}_{\mathrm{A}} \mathbf{H}^{T}-\mathbf{H} \boldsymbol{\Lambda}_{\mathrm{B}} \boldsymbol{\Lambda}_{\mathrm{C}}^{-1} \boldsymbol{\Lambda}_{\mathrm{B}}^{T} \mathbf{H}^{T}\right)^{-1}
$$

where we, to simplify the notation, define the Equivalent FIM (EFIM) $\mathbf{J}_{\mathbf{p}}$ as

$$
\mathbf{J}_{\mathbf{p}}=\mathbf{H}\left(\boldsymbol{\Lambda}_{\mathrm{A}}-\boldsymbol{\Lambda}_{\mathrm{B}} \boldsymbol{\Lambda}_{\mathrm{C}}^{-1} \boldsymbol{\Lambda}_{\mathrm{B}}^{T}\right) \mathbf{H}^{T}
$$

so that $\mathbf{J}_{p}^{-1}=\left[\mathbf{J}_{\theta}^{-1}\right]_{2 \times 2}$.

This now allows us to introduce and calculate the key performance metric used in this chapter: The positioning error bound (PEB) with respect to the agent position $\mathbf{x}$, which is defined as

$$
\operatorname{PEB}(\mathbf{x})=\sqrt{\operatorname{trace}\left\{\mathbf{J}_{p}^{-1}\right\}}=\sqrt{\operatorname{trace}\left\{\left[\mathbf{J}_{\theta}^{-1}\right]_{2 \times 2}\right\}} .
$$

We can also define the signal-to-interference-plus-noise-ratio (SINR) vector SINR $\in \mathbb{R}^{K}$ as

$$
[\mathbf{S I N R}]_{i}=[w]_{i}^{2} \frac{[\alpha]_{i}^{2}}{N_{0}}=\frac{[\alpha]_{i}^{2}}{N_{0}+T_{s} S_{v}\left([\boldsymbol{\tau}]_{i}\right)} .
$$

In the following subsections, we highlight some particular aspects - all of which are evaluated numerically later in Section 6.2.

\subsubsection{Impact of Path Overlap}

A discussed in [29], we can treat the path overlap, which is the impact of MPCs having similar propagation delay and hence arrive almost at the same time to the receiver, in three distinct ways:

Orthogonal where all MPCs are assumed orthogonal/resolvable. Hence, we set all off-diagonal elements of $\Lambda_{\mathrm{A}}$ to zero, and assume $\Lambda_{\mathrm{B}}^{\mathrm{R}}=\boldsymbol{\Lambda}_{\mathrm{B}}^{\mathrm{I}}=\mathbf{0}$ in (6.7). This will yield an optimistic, lower limit on the PEB.

With Overlap which is the full model just as described in the previous section.

No Overlap where any overlapping MPCs, i.e., those with $\left|\tau_{m}^{(k)}-\tau_{m}^{\left(k^{\prime}\right)}\right|<T_{s}$ for $k \neq k^{\prime}$, are discarded. This will yield an pessimistic, upper limit on the PEB. 


\subsubsection{Limited Observations}

It is not realistic to assume that all $K_{m}$ MPCs of anchor $m$ can be measured. In this thesis, we therefore investigate the case when only a subset of these are measured. This means that, although all $K_{m}$ MPCs will potentially cause interference/pathoverlap as discussed earlier, we only get the benefit of information from including the measured MPCs of a set we denote $\mathcal{K}_{m}$. A heuristic and greedy approach to construct the set $\mathcal{K}_{m}$, is to assume that we only observe the $K_{\text {MAX MPCs which }}$ maximizes a given measure $f\left(\tau_{m}^{(k)}, \alpha_{m}^{(k)}\right)$. This can be expressed iteratively as

$$
\begin{aligned}
\mathcal{K}_{m}^{1} & =\underset{k=1, \ldots, K_{m}}{\arg \max } f\left(\tau_{m}^{(k)}, \alpha_{m}^{(k)}\right) \\
\mathcal{K}_{m}^{p} & =\mathcal{K}_{m}^{p-1} \bigcup \underset{\substack{k=1, \ldots, K_{m} \\
k \notin \mathcal{K}_{m}^{p-1}}}{\arg \max } f\left(\tau_{m}^{(k)}, \alpha_{m}^{(k)}\right) \quad \forall p=2, \ldots, K_{\mathrm{MAX}}
\end{aligned}
$$

whereafter we have our desired observation set as $\mathcal{K}_{m}^{K_{\mathrm{MAx}}}$. Different variants of the measure $f\left(\tau_{m}^{(k)}, \alpha_{m}^{(k)}\right)$ that could be foreseen include

Max Amplitude where we simply select the MPC with the maximum amplitude $f\left(\tau_{m}^{(k)}, \alpha_{m}^{(k)}\right)=\left|\alpha_{m}^{(k)}\right|^{2}$.

Max SINR where we select the MPC with the highest SINR, which also takes the DM interference into account, $f\left(\tau_{m}^{(k)}, \alpha_{m}^{(k)}\right)=\frac{\left|\alpha_{m}^{(k)}\right|^{2}}{N_{0}+T_{s} S_{v}\left(\tau_{m}^{(k)}\right)}($ as per (6.12)).

We could further consider finding the first $K_{\text {MAX }}$ MPCs within a certain threshold from either of the above criteria, e.g., change

$$
\underset{k=1, \ldots, K_{m}}{\arg \max } f\left(\tau_{m}^{(k)}, \alpha_{m}^{(k)}\right)
$$

of (6.13) into

$$
\underset{k=1, \ldots, K_{m}}{\arg \min }\left\{\tau_{m}^{(k)}: f\left(\tau_{m}^{(k)}, \alpha_{m}^{(k)}\right) \geq \max \left(f\left(\tau_{m}^{\left(k^{\prime}\right)}, \alpha_{m}^{\left(k^{\prime}\right)}\right)\right)-T\right\}
$$

for some threshold $T$.

In this work we will however keep it simple and instead use what we refer to as a Limited Reflection Order model where we define

$$
\mathcal{K}_{m}=\left\{k: Q^{(k)} \leq Q_{\max }, k=1, \ldots, K_{m}\right\} \text { for } Q_{\max }=0,1,2,3
$$

so that, e.g., with $Q_{\max }=0$ only the LOS component is included, with $Q_{\max }=1$ both the LOS as well as the first order reflections are included and so on. 


\subsubsection{Separate DL and UL Measurements}

We start in the context of uni-directional measurements (see Section 3.3.1), i.e., with transmissions and measurements in one direction only. In the DL case, we assume that the transmissions from different anchors are identifiable at the agent. This is true if these are multiplexed in time, frequency or by means of choosing orthogonal reference signals which is what is assumed here. The different MPCs resulting from the transmission by one and the same anchor may however overlap as discussed earlier. Hence, we follow the procedure described earlier, but form the involved matrices on a per-direction and per-anchor basis which gives us $(6.10)$ as

$$
\mathbf{J}_{\mathbf{p}, m}^{\mathrm{DL}}=\mathbf{H}_{m}^{\mathrm{DL}}\left(\boldsymbol{\Lambda}_{\mathrm{A}, m}^{\mathrm{DL}}-\boldsymbol{\Lambda}_{\mathrm{B}, m}^{\mathrm{DL}}\left(\boldsymbol{\Lambda}_{\mathrm{C}, m}^{\mathrm{DL}}\right)^{-1}\left(\boldsymbol{\Lambda}_{\mathrm{B}, m}^{\mathrm{DL}}\right)^{T}\right)\left(\mathbf{H}_{m}^{\mathrm{DL}}\right)^{T}
$$

for each anchor $m=1, \ldots, M$, where the definition of the involved matrices are as per (6.6) and (6.7). Under the assumption that the measurements with respect to the different anchors are independent and hence that the information is additive (see e.g. [16]) the joint DL EFIM for over the different anchors is

$$
\mathbf{J}_{\mathbf{p}}^{\mathrm{DL}}=\sum_{m=1}^{M} \mathbf{J}_{\mathbf{p}, m}^{\mathrm{DL}}
$$

after which we can use (6.11) to calculate the PEB in the DL. Another way to see it is as in the MPCs from the same anchor are dealt with as with overlap (see earlier discussion in Section 6.1.1) whereas from different anchors the MPCs are treated as orthogonal.

For the UL case, the situation is very similar to that of the DL discussed in above and we can form a similar expression as per (6.15):

$$
\mathbf{J}_{\mathbf{p}, m}^{\mathrm{UL}}=\mathbf{H}_{m}^{\mathrm{UL}}\left(\boldsymbol{\Lambda}_{\mathrm{A}, m}^{\mathrm{UL}}-\boldsymbol{\Lambda}_{\mathrm{B}, m}^{\mathrm{UL}}\left(\boldsymbol{\Lambda}_{\mathrm{C}, m}^{\mathrm{UL}}\right)^{-1}\left(\boldsymbol{\Lambda}_{\mathrm{B}, m}^{\mathrm{UL}}\right)^{T}\right)\left(\mathbf{H}_{m}^{\mathrm{UL}}\right)^{T}
$$

where in this case we have

$$
\mathbf{H}_{m}^{\mathrm{UL}}=\frac{1}{c}\left[\begin{array}{lll}
\cos \gamma^{(1)} & \cdots & \cos \gamma^{\left(K_{m}\right)} \\
\sin \gamma^{(1)} & \cdots & \cos \gamma^{\left(K_{m}\right)}
\end{array}\right],
$$

so that its $k$ th column is given by $\frac{1}{c}\left[\cos \gamma^{(k)} \sin \gamma^{(k)}\right]^{T}$ where $\gamma^{(k)}$ is now the UL angle-of-arrival for MPC $k$ sent by the agent and received by anchor $m$. In general, this has no direct relation to the placement of the VA, but instead can be expressed as the angle from the last wall-bounce to the MPC to the anchor, i.e.,

$$
\gamma_{m}^{(k)}=\tan ^{-1} \frac{y_{w}-p_{y, m}}{x_{w}-p_{x, m}} .
$$

where thus $\left[x_{w}, y_{w}\right]$ are the coordinates of the last position of the last wall the UL MPC was reflected before reaching the anchor. Clearly, this is the same as the angle of departure for the corresponding MPC in the DL but rotated by $\pi$ radians. The joint UL EFIM over the $\mathrm{M}$ anchors becomes

$$
\mathbf{J}_{\mathbf{p}}^{\mathrm{UL}}=\sum_{m=1}^{M} \mathbf{J}_{\mathbf{p}, m}^{\mathrm{UL}} .
$$




\subsubsection{Combined DL and UL Measurements}

Consider now instead a bi-directional measurement scenario as per Section 3.3.2. Regardless of which approach discussed therein is used, we can define a parameter vector containing both uplink and downlink measurements as

$$
\psi^{\mathrm{TOT}}=\left[\begin{array}{l}
\psi^{\mathrm{DL}} \\
\psi^{\mathrm{UL}}
\end{array}\right] \in \mathbb{R}^{3 K_{\mathrm{DL}}+3 K_{\mathrm{UL}}}
$$

where we need not include the processing delay $\Delta$ which is assumed known.

Under the assumption that the measurements in UL and DL are independent due to the different noise realizations, we get a block-diagonal alternative FIM as

$$
\mathbf{J}_{\psi}^{\mathrm{TOT}}=\left[\begin{array}{cc}
\mathbf{J}_{\psi}^{\mathrm{DL}} & \mathbf{0} \\
\mathbf{0} & \mathbf{J}_{\psi}^{\mathrm{UL}}
\end{array}\right]
$$

whereas the Jacobian transformation matrix straightforwardly becomes

$$
\mathbf{T}^{\mathrm{TOT}}=\frac{\partial \psi^{\mathrm{TOT}}}{\partial \boldsymbol{\theta}}=\left[\begin{array}{ll}
\frac{\partial \psi^{\mathrm{DL}}}{\partial \boldsymbol{\theta}} & \frac{\partial \psi^{\mathrm{UL}}}{\partial \boldsymbol{\theta}}
\end{array}\right]=\left[\begin{array}{ll}
\mathbf{T}^{\mathrm{DL}} & \mathbf{T}^{\mathrm{UL}}
\end{array}\right] .
$$

Hence the resulting FIM is given by

$$
\begin{aligned}
\mathbf{J}_{\theta}^{\mathrm{TOT}} & =\mathbf{T}^{\mathrm{TOT}} \mathbf{J}_{\psi}^{\mathrm{TOT}}\left(\mathbf{T}^{\mathrm{TOT}}\right)^{T}=\left[\begin{array}{ll}
\mathbf{T}^{\mathrm{DL}} & \mathbf{T}^{\mathrm{UL}}
\end{array}\right]\left[\begin{array}{cc}
\mathbf{J}_{\psi}^{\mathrm{DL}} & \mathbf{0} \\
\mathbf{0} & \mathbf{J}_{\psi}^{\mathrm{UL}}
\end{array}\right]\left[\begin{array}{c}
\left(\mathbf{T}^{\mathrm{DL}}\right)^{T} \\
\left(\mathbf{T}^{\mathrm{UL}}\right)^{T}
\end{array}\right] \\
& =\mathbf{T}^{\mathrm{DL}} \mathbf{J}_{\psi}^{\mathrm{DL}}\left(\mathbf{T}^{\mathrm{DL}}\right)^{T}+\mathbf{T}^{\mathrm{UL}} \mathbf{J}_{\psi}^{\mathrm{UL}}\left(\mathbf{T}^{\mathrm{UL}}\right)^{T}=\mathbf{J}_{\boldsymbol{\theta}}^{\mathrm{DL}}+\mathbf{J}_{\boldsymbol{\theta}}^{\mathrm{UL}}
\end{aligned}
$$

and, more explicitly with respect to the top-left $2 \times 2$ sub-block of $\mathbf{J}_{\theta}^{\mathrm{TOT}}$ relating to the agent position $\mathbf{x}$, we get

$$
\left[\mathrm{J}_{\theta}^{\mathrm{TOT}}\right]_{2 \times 2}=\left[\mathrm{J}_{\theta}^{\mathrm{DL}}\right]_{2 \times 2}+\left[\mathrm{J}_{\theta}^{\mathrm{UL}}\right]_{2 \times 2} \text {. }
$$

This means that we have additivity in the information, which is what could be expected under the earlier independence assumptions (see e.g. [16]). We can now use (6.11) together with (6.25) to calculate the PEB also in this scenario.

Note that, in the case of combined DL and UL observations, the same configuration need not necessarily be applied in both directions. For example, the agent could be configured to measure and report only one MPC (found using either of the above approaches) whereas the anchors still measure all MPCs in the UL.

\subsection{Numerical Results}

Evaluations in terms of the PEB of Section 6.1 as defined in (6.11) are performed for both Scenario A and B described in Section 2.1. This also includes the circular trajectory of Scenario A depicted in Figure 2.2, for which what is denoted as 'Position Along Trajectory' in Figures 6.1, 6.4 and 6.7 of this chapter is nothing but the angle in degrees counted counter-clockwise relative the negative $y$-axis, where the agent started, as indicated in Figure 2.2.

The different aspects discussed in the subsections of Section 6.1 are evaluated in separate subsections also here. 


\subsubsection{Impact of Path Overlap}

We start by evaluating the impact of path overlap as discussed in Section 6.1.1. Results using DL measurements for Scenario A are presented for the circular trajectory in Figure 6.1 and over the $5 \times 5 \mathrm{~cm}$ grid in Figure 6.2, which shows:

- As discussed earlier, the 'Orthogonal' model indeed gives an optimistic lower PEB bound, whereas the 'No Overlap' model gives a more pessimistic upper РEB bound. The full 'With Overlap' model give more realistic results somewhere in-between the other two models.

- The positioning accuracy is better when using all anchors compared to only one. In particular, the outage regions, where not enough information is available, are significantly reduced when utilizing multiple anchors.

- There are regions which are inherently difficult for all models and/or combination of anchors, and other regions where the choice of model and/or combination of anchors makes a large difference.

The corresponding gridded results for Scenario B in Figure 6.3 shows us that:

- The difference between the models is much smaller in this scenario. There are also some banded structures at the positions where symmetries in the room geometry causes 'fading-dips' where the different MPCs cancel each other out, as discussed in e.g. [7].

- The positioning accuracy is quite poor when using only one anchor. When using all anchors, it is on the other hand quite good.

Both observations for Figure 6.3 can be explained by the fact that in Scenario B, the received energy contained in the non-LOS MPCs is quite small compared to the LOS MPC since the room is quite large and hence that the path-loss is more pronounced as compared to Scenario A. In essence, this makes the positioning information in Scenario B become almost solely based on LOS information and hence the inclusion of more anchors increases this information.

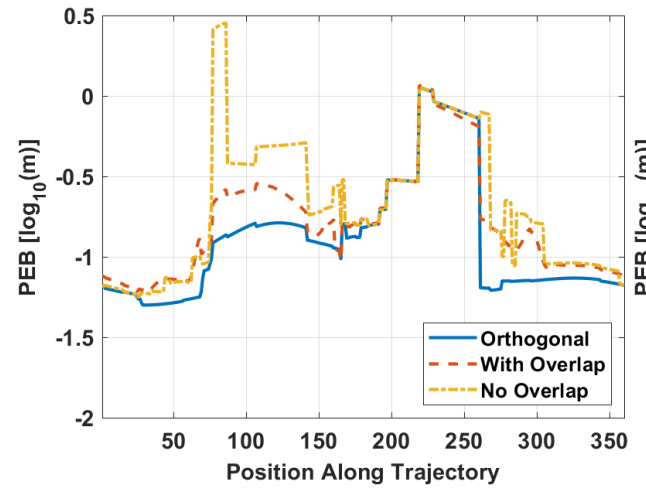

(a) Single Anchor

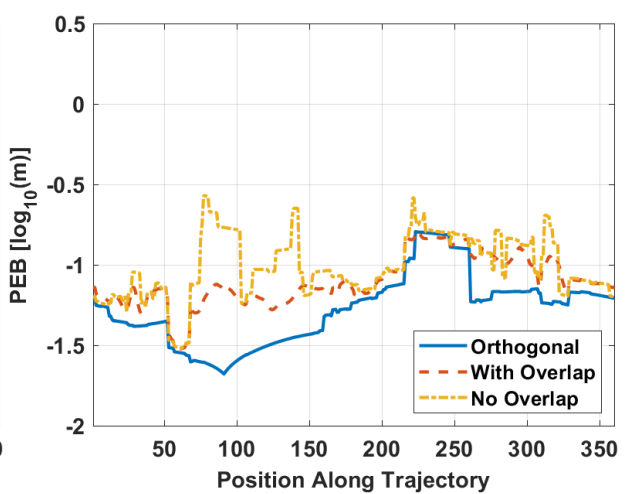

(b) All Anchors

Figure 6.1: The PEB over the circular trajectory for Scenario A. 

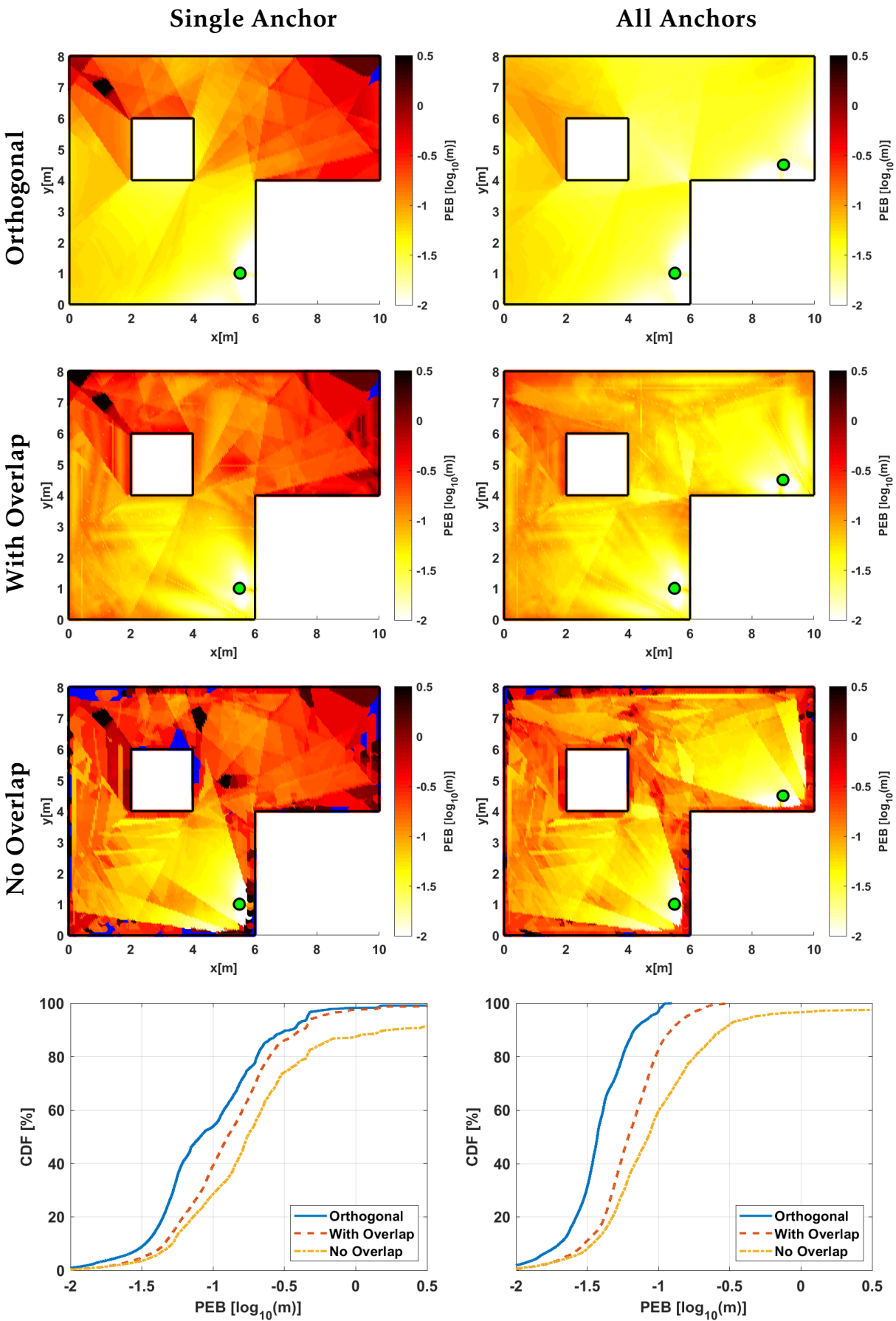

Figure 6.2: The PEB over the entire room for of Scenario A for transmissions to/from one anchor (left column) and all anchors (right column) where blue regions indicate where the EFIM is rank-deficient and hence positioning is not possible. The last row shows the CDF for the different cases. 
Single Anchor
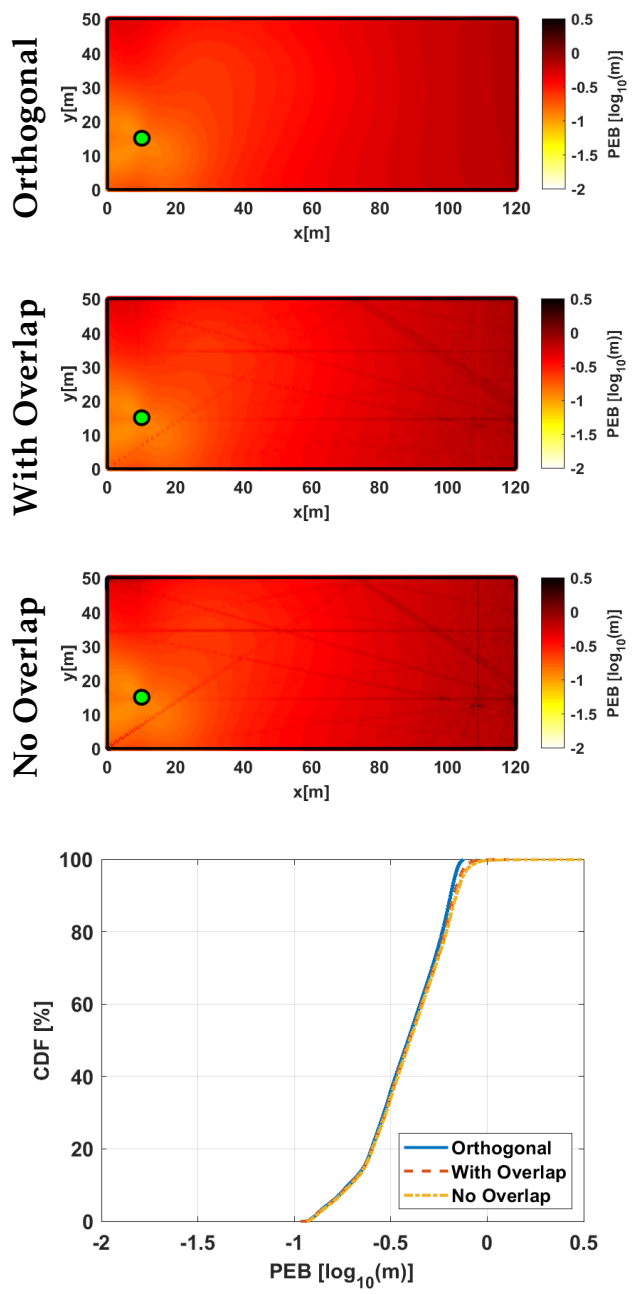

All Anchors
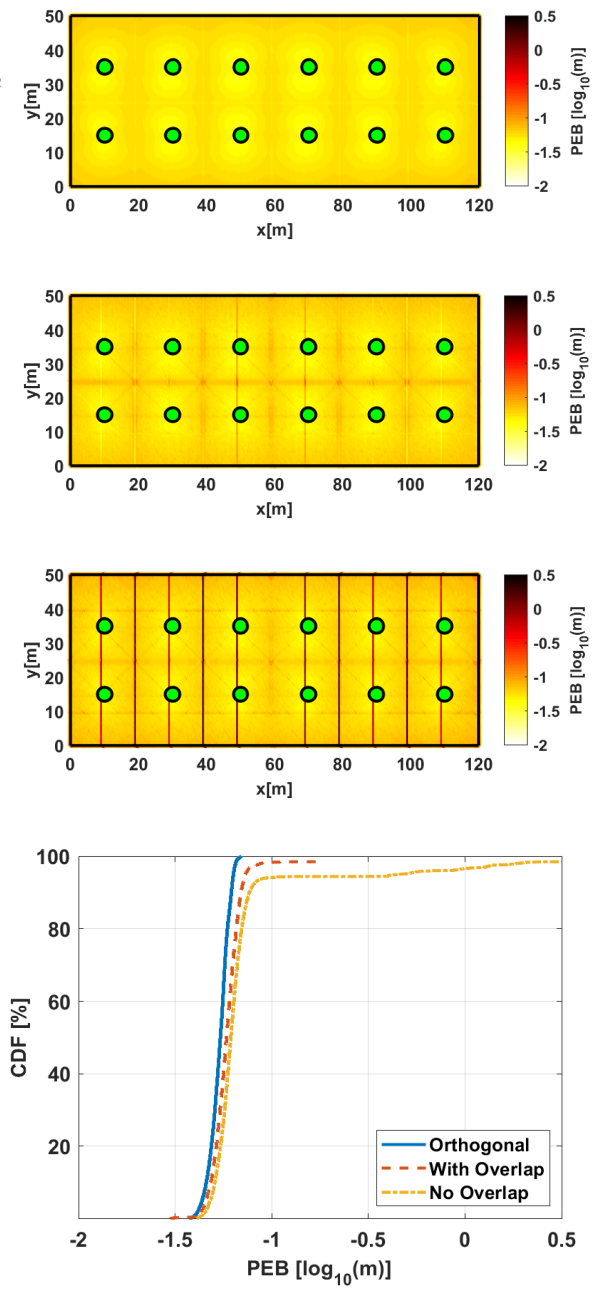

Figure 6.3: Same as Figure 6.2 but for Scenario B. 


\subsubsection{Limited Observations}

Next, we investigate the impact of limiting the number of observations as discussed in Section 6.1.2. More precisely, we use the limited reflection order model (6.14) and assume the 'With Overlap' model discussed in Sections 6.1.1 and 6.2.1. The results for Scenario A are shown in Figures 6.4 and 6.5 which tells us that:

- Using only information from the LOS MPC components by $Q_{\max }=0$ does not give enough information for positioning regardless if considering one or all anchors, unless having LOS to both anchors in the latter case.

- With measurements from one single anchor, satisfactory positioning accuracy can be achieved in almost all regions if accounting for up to the thirdorder reflections with $Q_{\max }=3$. As expected, the accuracy is worse in the non-LOS regions.

- Using all anchors, the accuracy is good already when including secondorder reflections $\left(Q_{\max }=2\right)$, and with minimal additional benefit if including also the third-order reflections. With only first-order reflections $\left(Q_{\max }=1\right)$ included, the accuracy with non-LOS to both anchors is very poor, as e.g. between positions 220 and 260 in the trajectory of Figure 6.4.

For the Scenario B results in Figure 6.6 we see, like before, that

- If using measurements from one single anchor, using only information from the LOS MPCs with $Q_{\max }=0$, does not give enough information for positioning at all. But even including higher order reflections, the performance is still quite poor - the reason for this being that the received power in the non-LOS MPCs is quite low (as discussed in Section 6.2.1).

- Using all anchors, gives very good performance already using only the LOS MPC components with $Q_{\max }=0$. All the different anchors contribute with information in this case (again as in Section 6.2.1).

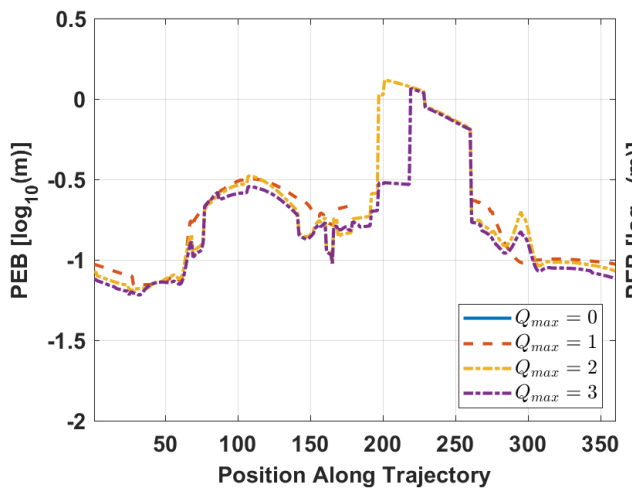

(a) Single Anchor

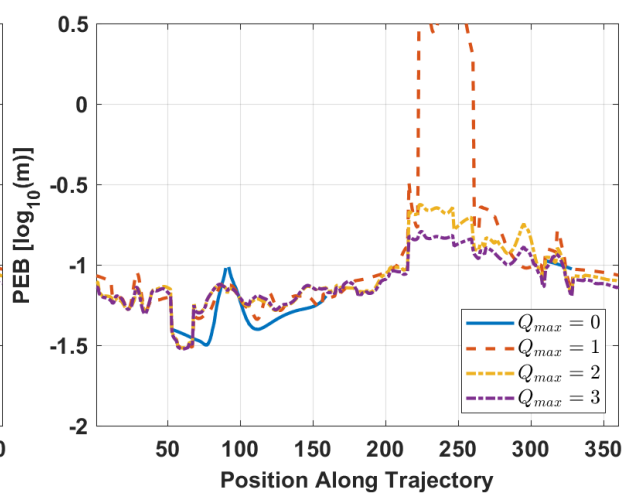

(b) All Anchors

Figure 6.4: The PEB over the circular trajectory for Scenario A. 

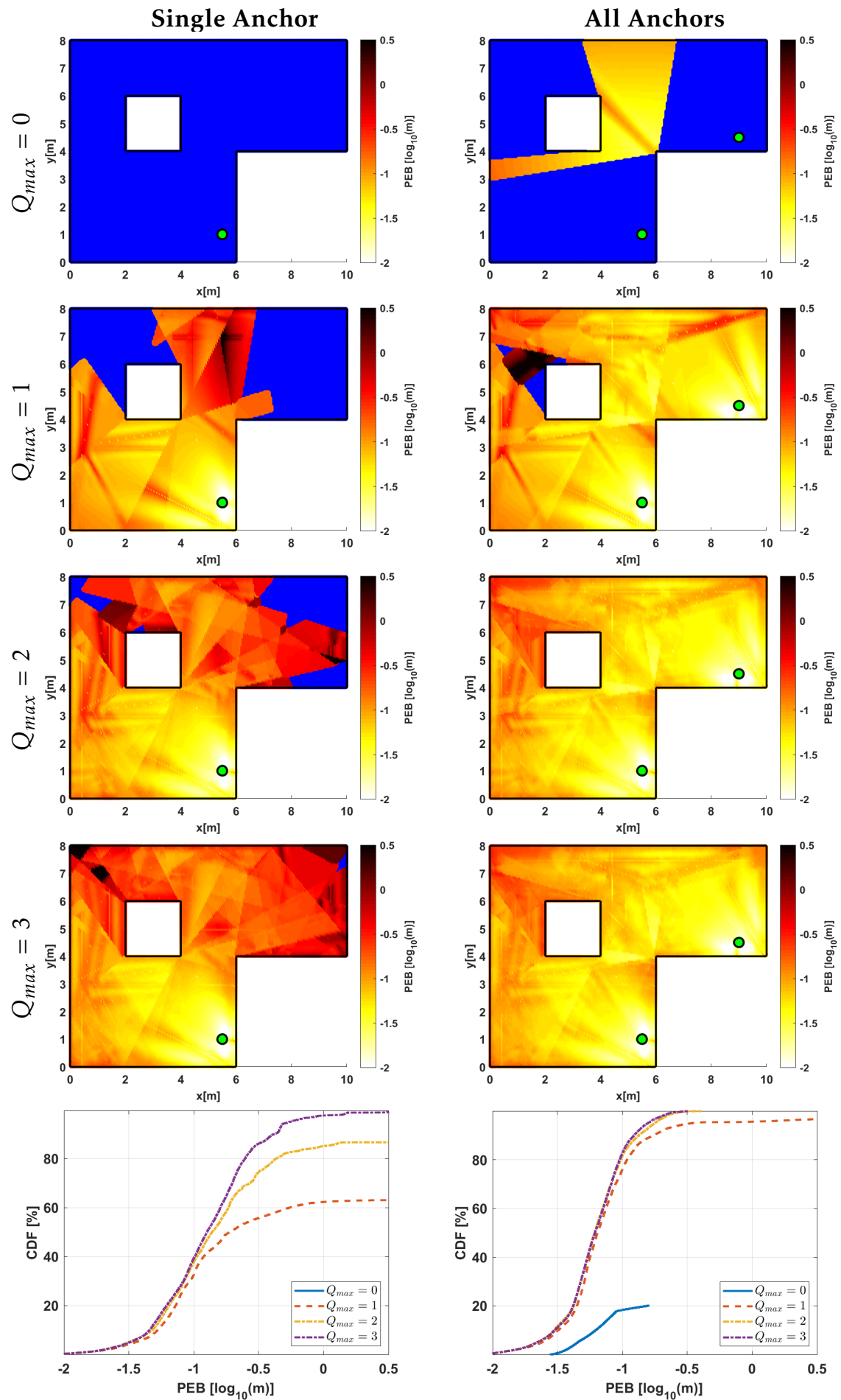

Figure 6.5: The PEB over the entire room of Scenario A for varying assumptions on the maximum number of reflections $Q_{\max }$ for the included MPCs. 
Single Anchor
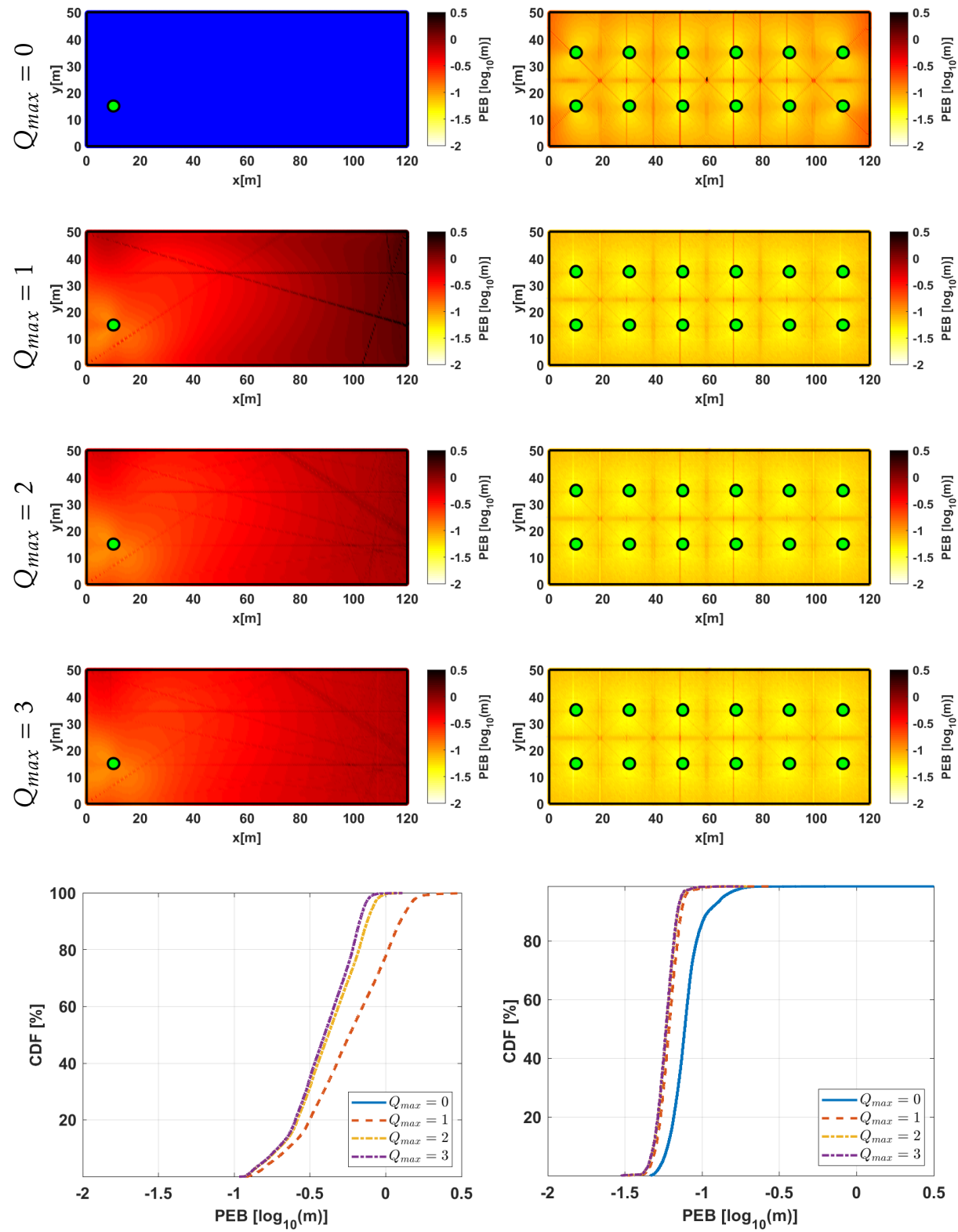

All Anchors
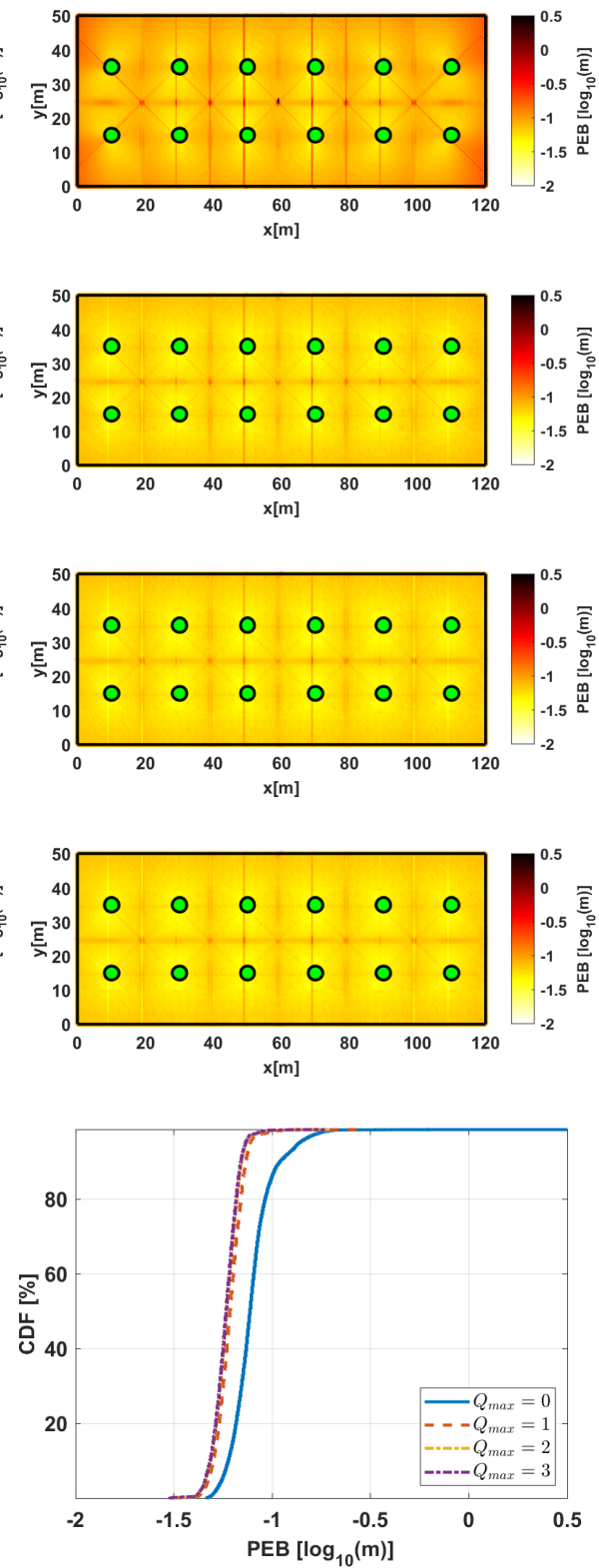

Figure 6.6: Same as Figure 6.5 but for Scenario B. 


\subsubsection{Combined DL and UL Measurements}

Finally, we investigate the impact of combining DL and UL measurements as discussed in Section 6.1.4. Again we assume the 'With Overlap' model as discussed in Sections 6.1.1 and 6.2.1 and use the limited reflection order model of (6.14). Here, we vary the measured reflection order independently in the respective directions so that e.g. the combination $Q_{\max }^{D L}=0$ and $Q_{\max }^{U L}=2$ means that the agent measures and reports only the LOS MPC, whereas the anchor(s) measures both LOS as well as first- and second-order reflected MPCs.

The results for the trajectory of Scenario A is given in Figure 6.7. The grid results over the entire room when utilizing only one anchor is given in Figure 6.8 and when utilizing all anchors is given in Figure 6.10. The corresponding CDF plots are shown in Figure 6.9 and the results summarized in Table 6.1. Based on these results we conclude that

- If using measurements from one single anchor, we need to include essentially all MPC in at least one of the directions to get satisfactory performance, thus having $Q_{\max }^{D L}=3$ and/or $Q_{\max }^{U L}=3$.

- Using all anchors however, gives very good performance already using only the second-order MPCs in either direction, i.e., $Q_{\max }^{D L}=2$ and/or $Q_{\max }^{U L}=2$. The benefit of including even higher order reflections is very limited.

The corresponding results for Scenario B are given in Figures 6.11, 6.12 and 6.13. As before, we see that

- Using measurements from one single anchor, positioning performance is poor regardless of how many MPCs are included in both directions.

- Using all anchors gives good performance already using only the LOS MPC components, i.e., having $Q_{\max }^{D L}=Q_{\max }^{U L}=0$. Adding the first-order reflections in either direction gives some additional gain, but beyond that the gain is very limited.

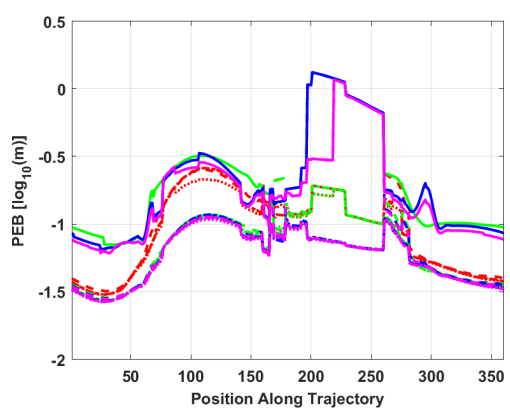

(a) Single Anchor

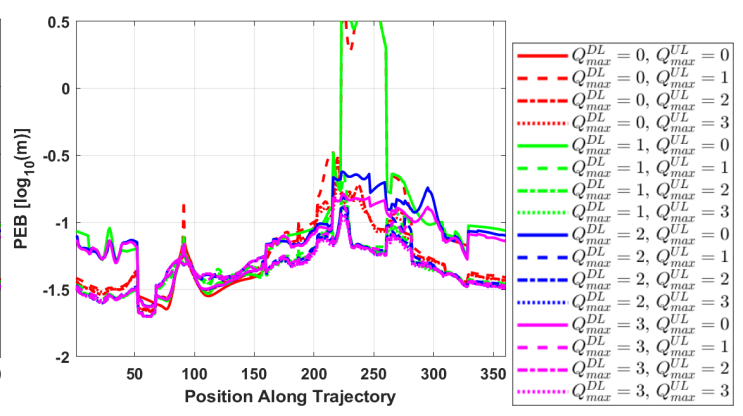

(b) All Anchors

Figure 6.7: The PEB over the circular trajectory for Scenario A. 
$Q_{\max }^{U L}=0$
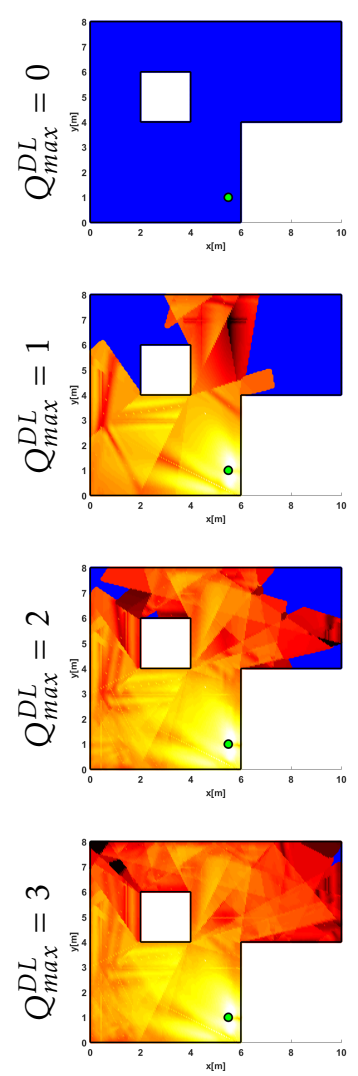

$Q_{\max }^{U L}=1$
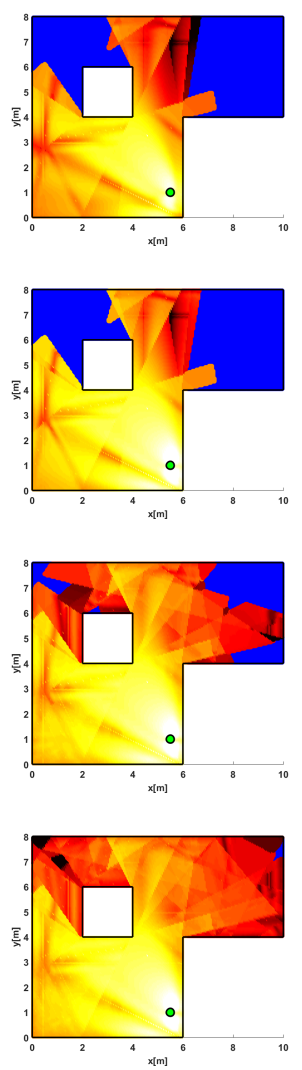

$Q_{\max }^{U L}=2$
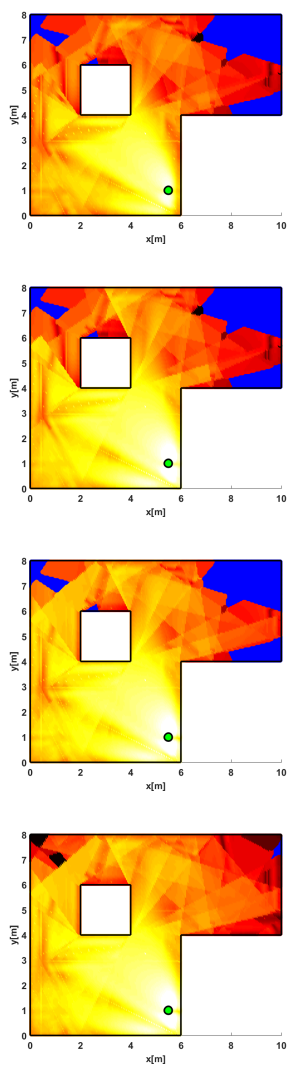

$Q_{\max }^{U L}=3$
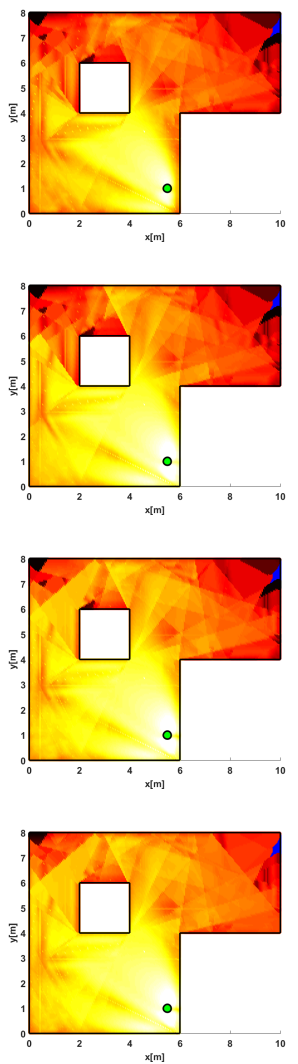

Figure 6.8: РЕB over the entire room for Scenario A with UL and DL transmissions of one anchor.

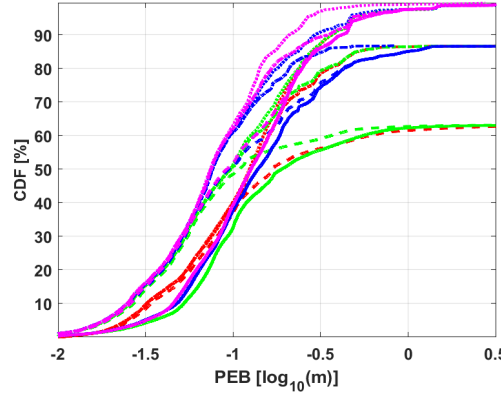

(a) Single Anchor

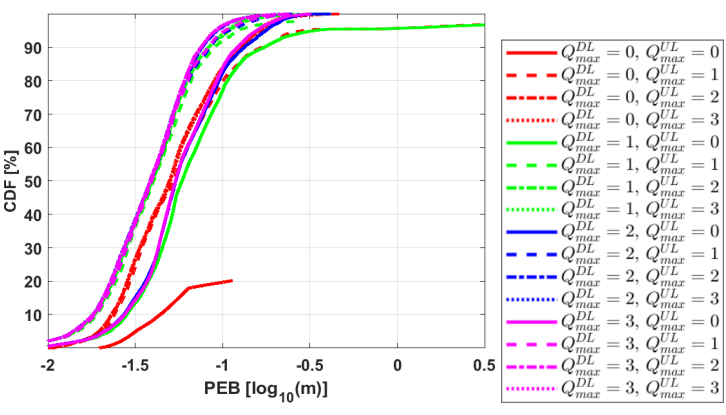

(b) All Anchors

Figure 6.9: $P E B$ CDFs over the entire room for Scenario A. 


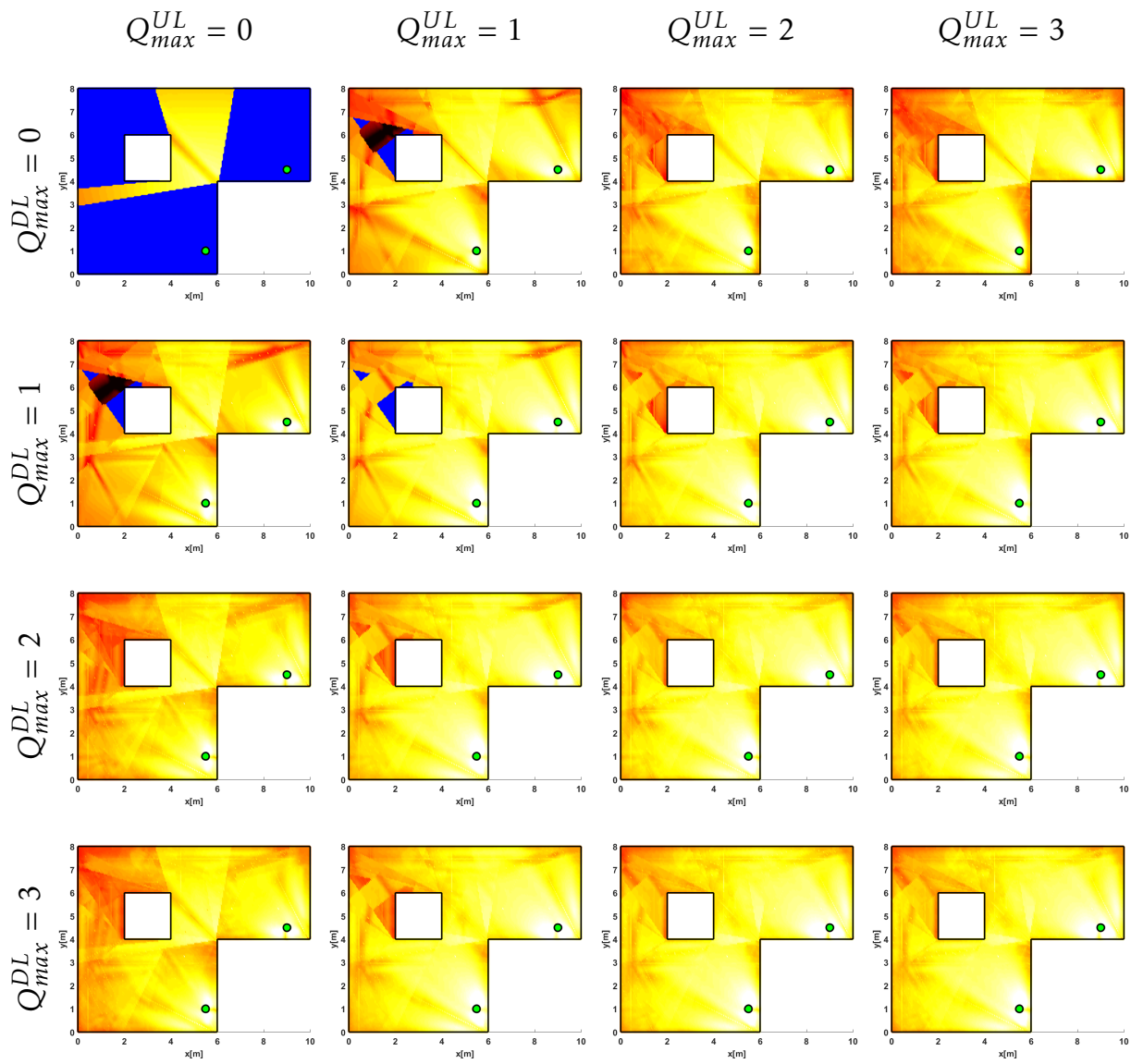

Figure 6.10: Same as Figure 6.8 but with UL and DL transmissions of all anchors.

Table 6.1: The $10^{\text {th }} / 50^{\text {th }} / 90^{\text {th }}$ percentiles of the PEB $[\mathrm{m}]$ over the entire room for Scenario $A$, where $\infty$ means that positioning was not possible.

\begin{tabular}{cc|ccccc} 
& & & \multicolumn{4}{|c}{$Q_{\max }^{U L}$} \\
& & & 0 & 1 & 2 & 3 \\
\hline \multirow{4}{*}{ Single Anchor } & & 0 & $\infty / \infty / \infty$ & $0.03 / 0.17 / \infty$ & $0.03 / 0.13 / \infty$ & $0.03 / 0.12 / 0.37$ \\
& $Q_{\max }^{D L}$ & 1 & $0.05 / 0.19 / \infty$ & $0.03 / 0.11 / \infty$ & $0.03 / 0.10 / \infty$ & $0.03 / 0.10 / 0.37$ \\
& & $0.05 / 0.14 / \infty$ & $0.03 / 0.09 / \infty$ & $0.03 / 0.08 / \infty$ & $0.02 / 0.08 / 0.29$ \\
& & $0.05 / 0.13 / 0.41$ & $0.03 / 0.09 / 0.41$ & $0.02 / 0.07 / 0.35$ & $0.02 / 0.07 / 0.21$ \\
\hline \multirow{4}{*}{ All Anchors } & & 0 & $0.04 / \infty / \infty$ & $0.02 / 0.05 / 0.17$ & $0.02 / 0.05 / 0.13$ & $0.02 / 0.05 / 0.13$ \\
& $Q_{\max }^{D L}$ & 1 & $0.03 / 0.06 / 0.17$ & $0.02 / 0.04 / 0.09$ & $0.02 / 0.04 / 0.08$ & $0.02 / 0.04 / 0.08$ \\
& 2 & $0.03 / 0.05 / 0.13$ & $0.02 / 0.04 / 0.08$ & $0.02 / 0.04 / 0.08$ & $0.02 / 0.04 / 0.08$ \\
& & 3 & $0.03 / 0.05 / 0.12$ & $0.02 / 0.04 / 0.08$ & $0.02 / 0.04 / 0.07$ & $0.02 / 0.04 / 0.08$ \\
\hline
\end{tabular}




$$
Q_{\max }^{U L}=0 \quad Q_{\max }^{U L}=1 \quad Q_{\max }^{U L}=2 \quad Q_{\max }^{U L}=3
$$
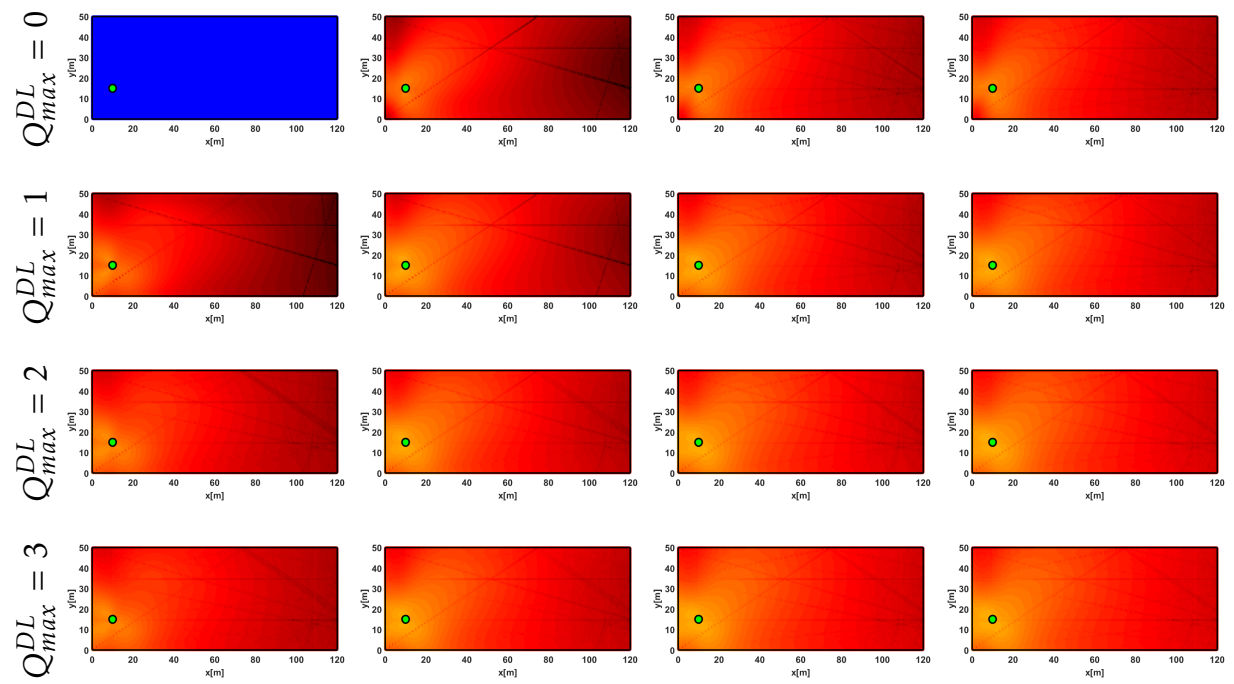

Figure 6.11: Same as Figure 6.8 but for Scenario B.

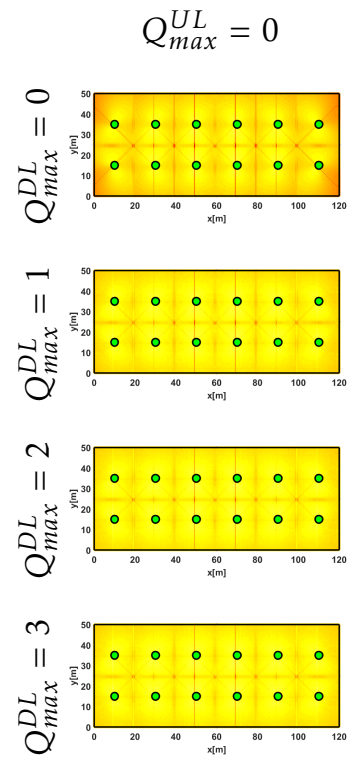

$$
Q_{\max }^{U L}=1
$$$$
Q_{\max }^{U L}=2
$$$$
Q_{\max }^{U L}=3
$$
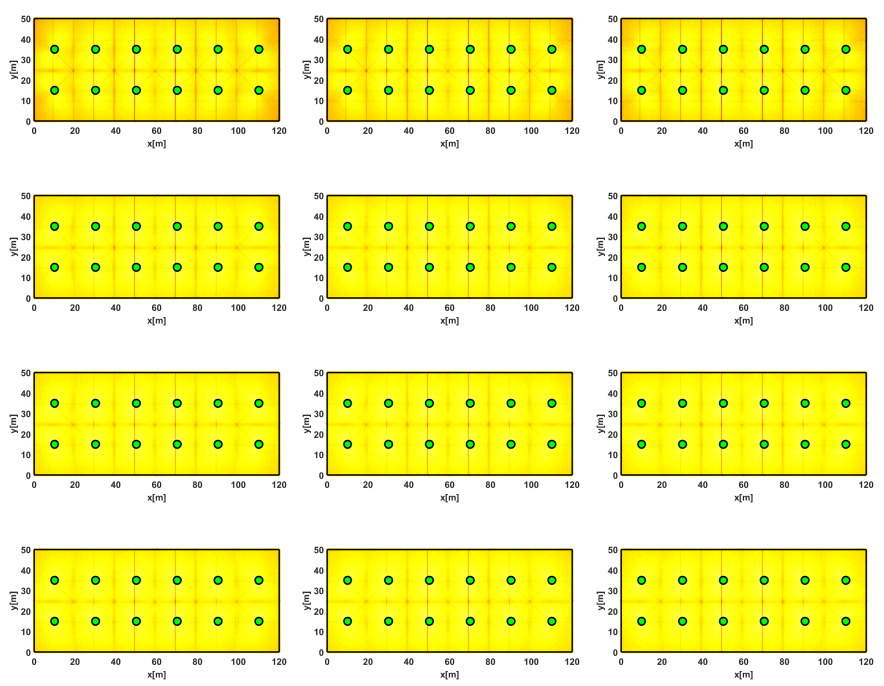

Figure 6.12: Same as Figure 6.10 but for Scenario B. 


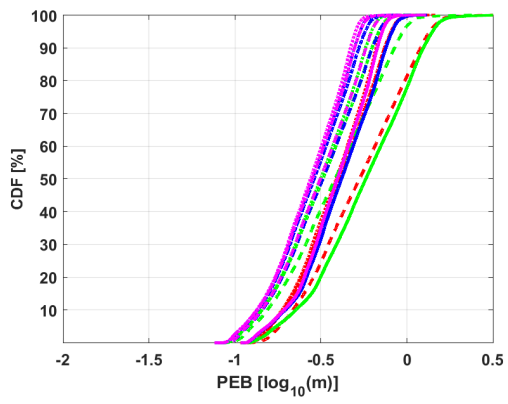

(a) Single Anchor

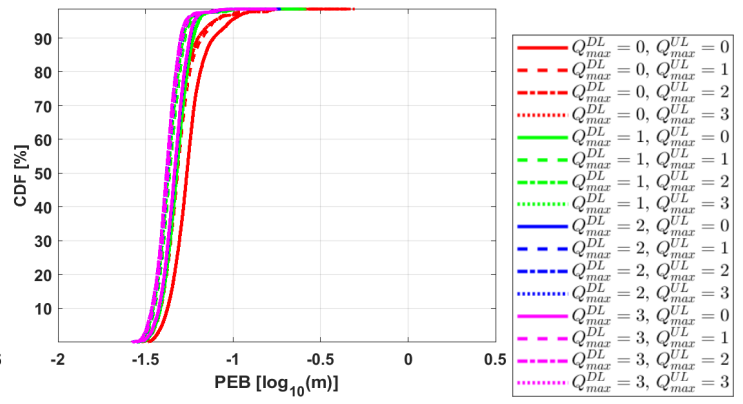

(b) All Anchors

Figure 6.13: Same as Figure 6.9 but for Scenario B.

Table 6.2: Same as Table 6.1 but for Scenario B.

\begin{tabular}{cc|ccccc} 
& & & \multicolumn{4}{|c}{$Q_{\max }^{U L}$} \\
& & & 0 & 1 & 2 & 3 \\
\hline \multirow{4}{*}{ Single Anchor } & $Q_{\max }^{D L}$ & 1 & $0.23 / 0.58 / 1.24$ & $0.15 / 0.38 / 0.85$ & $0.14 / 0.32 / 0.62$ & $0.14 / 0.31 / 0.57$ \\
& & 2 & $0.20 / 0.42 / 0.75$ & $0.14 / 0.32 / 0.63$ & $0.14 / 0.29 / 0.52$ & $0.13 / 0.28 / 0.49$ \\
& 3 & $0.19 / 0.39 / 0.67$ & $0.14 / 0.31 / 0.58$ & $0.13 / 0.28 / 0.49$ & $0.13 / 0.27 / 0.47$ \\
\hline \multirow{3}{*}{ All Anchors } & & 0 & $0.04 / 0.05 / 0.08$ & $0.04 / 0.05 / 0.07$ & $0.04 / 0.05 / 0.06$ & $0.04 / 0.05 / 0.06$ \\
& $Q_{\max }^{D L}$ & 1 & $0.04 / 0.05 / 0.06$ & $0.04 / 0.04 / 0.05$ & $0.03 / 0.04 / 0.05$ & $0.03 / 0.04 / 0.05$ \\
& 2 & $0.04 / 0.05 / 0.06$ & $0.03 / 0.04 / 0.05$ & $0.03 / 0.04 / 0.05$ & $0.03 / 0.04 / 0.05$ \\
& & 3 & $0.04 / 0.05 / 0.06$ & $0.03 / 0.04 / 0.05$ & $0.03 / 0.04 / 0.05$ & $0.03 / 0.04 / 0.05$ \\
\hline
\end{tabular}

\subsection{Summary}

We have formulated the theoretical bounds on positioning accuracy when utilizing multiple MPCs in the estimation process. Evaluations have been performed in scenarios with one agent and multiple anchors, where it has been shown that:

- The modelling of path overlap, i.e., how to deal with multiple MPCs that arrive approximately at the same time to the receiver, makes quite some difference in terms of positioning accuracy.

- The positioning accuracy depends on the room geometry as well as anchor deployment. A non-uniform room and/or deployment typically gives an accuracy which varies significantly over the room.

- A rich radio environment, i.e., with many rather strong received reflections of the transmitted signal is beneficial and allows for better positioning accuracy using fewer anchors. In contrast, in a less rich radio-environment where basically only the LOS MPCs are received, more anchors and/or a combination of DL and UL measurements are needed. 



\section{Concluding Remarks}

This chapter concludes the thesis by summarizing the results and discussing some possible directions for future work.

\subsection{Conclusions}

The key topic of this thesis has been how radio-based localization using timing/ranging measurements can be improved by using more of the information provided by measurements in a radio channel subject to multipath fading.

Chapters 2 and 3 started by introducing the general framework used throughout the thesis. Here a given signal model and a map of the surroundings were used to build a generic model of a multipath fading radio channel, from which the resulting MPCs were estimated.

Chapter 4 presented the first contribution of this thesis. It was shown how to mitigate the adverse effects of multipath propagation and non-LOS conditions for positioning based on estimation of the shortest (in a Euclidean sense) DP/LOS path between the transmitter and receiver. This was achieved with a set of robust statistical measurement error models, for which it was seen that models based on aggregated error statistics provided significant improvements without the need to provide detailed received signal information, but with a simpler and rather generic model. The gains were shown to be even larger with up-to-date real-time information based on the estimated MPCs.

Chapter 5 covered the second contribution of this thesis. The combinatorial problem of associating the estimated MPCs with the actual traversed signal paths was approached using methods from multi-target tracking. It was formulated as a classical assignment problem, accounting for erroneous measurements of various sorts, which was thereafter solved using a Global Nearest Neighbour (GNN) method. A rich radio environment in terms of many MPCs gave not only better 
localization accuracy, but also caused the association problem size to grow large - something which could be remedied by prior information allowing the exclusion of less probable paths. In many cases, especially in rich radio environments, simulations indicated the single best association hypothesis to be a reasonable approximation which avoided the calculation of a vast number of possible hypotheses. Accounting for erroneous measurements was seen crucial if such were present, otherwise it had some drawbacks.

Chapter 6 provided the third and final contribution of this thesis, which was the theoretical localization performance bounds when utilizing all or a subset of this multipath information, i.e., the MPCs. It was confirmed that a rich radio environment with many measurable MPCs allowed for good positioning accuracy using only few transmitters/receivers, assuming that these were indeed used in the localization process. In contrast, in a less rich environment were basically only the DP/LOS components were measurable, more transmitters/receivers and/or the combination of downlink and uplink measurements were required in order to achieve the same accuracy. The receiver's capability of distinguishing between multiple MPCs arriving approximately at the same time also affected the accuracy.

\subsection{Future Work}

The work of this thesis can be extended in a number of ways:

Even though we developed a MIMO channel model in Chapter 2, we still considered only a SISO model in the following chapters. It would be interesting to extend the concepts/evaluations also to a MIMO setting, which may also raise the question of AOA estimation and its usage in the subsequent parts of the thesis.

The measurement error models of Chapter 4 were more or less heuristically derived. A more theoretical approach to what indeed would be the optimal models is desirable. This includes re-visiting the choice of weights for the timedynamic 'Gaussian mixture' error model. Rather than using the received power of the respective MPC as done now, one should here instead factor in the probability of correctly estimating the DP.

The measurement association of Chapter 5 used a simple MPC estimation model, but should for completeness be done using the proper estimation methods described in Chapter 3. Furthermore, this chapter utilizes only the delay and not the amplitude estimates of the MPCs. This could also be included, which would essentially add one dimension to the hypotheses and hence render a more complex problem but, most likely, also give even better localization accuracy because of the increased amount of available information. This could however also be problematic since the channel amplitudes are typically less stable and more prone to disturbances than the pseudo-range measurements.

Finally, throughout this thesis we assumed the geometry of the environment to be known a priori through e.g. a map, after which we tried to estimate the position of an object based on this. One could turn the problem around to attempt to e.g. assess how accurate the mapping can be done given measurements of anchors and agents with a priori known positions. 


\section{Bibliography}

[1] 3GPP. Study on NR positioning support. Technical Specification (TS) 38.855, 3rd Generation Partnership Project (3GPP), Mar. 2019. Version 16.0.0.

[2] T. Bailey and H. Durrant-Whyte. Simultaneous localization and mapping (SLAM): Part II. IEEE Robotics Automation Magazine, 13(3):108-117, Sept. 2006.

[3] Y. Bar-Shalom, P. K. Willett, and X. Tian. Tracking and Data Fusion: A Handbook of Algorithms. Yaakov Bar-Shalom, Storrs, Conn, Apr. 2011.

[4] A. Bergström, G. Hendeby, F. Gunnarsson, and F. Gustafsson. TOA estimation improvements in multipath environments by measurement error models. In 2017 IEEE 28th Annual International Symposium on Personal, Indoor, and Mobile Radio Communications (PIMRC), pages 1-7, Montreal, QC, CA, Oct. 2017.

[5] S. Blackman and R. Popoli. Design and Analysis of Modern Tracking Systems. Artech House Publishers, June 1999.

[6] D. Dardari, C. C. Chong, and M. Win. Threshold-Based Time-of-Arrival Estimators in UWB Dense Multipath Channels. IEEE Transactions on Communications, 56(8):1366-1378, Aug. 2008.

[7] G. Dumphart. Performance Bounds for Anchorless Cooperative Indoor Localization Exploiting Multipath. Master's thesis, Graz University of Technology, 2014.

[8] H. Durrant-Whyte and T. Bailey. Simultaneous localization and mapping: Part I. IEEE Robotics Automation Magazine, 13(2):99-110, June 2006.

[9] C. Falsi, D. Dardari, L. Mucchi, and M. Z. Win. Time of Arrival Estimation for UWB Localizers in Realistic Environments. EURASIP Journal on Advances in Signal Processing, 2006(1), 2006. 
[10] C. Fritsche, A. Klein, and F. Gustafsson. Bayesian Cramer-Rao Bound for Mobile Terminal Tracking in Mixed LOS/NLOS Environments. IEEE Wireless Communications Letters, 2(3):335-338, June 2013.

[11] M. Froehle, P. Meissner, and K. Witrisal. Tracking of UWB multipath components using probability hypothesis density filters. In 2012 IEEE International Conference on Ultra-Wideband, pages 306-310, Syracuse, NY, USA, Sept. 2012.

[12] S. Gezici, Z. Tian, G. B. Giannakis, H. Kobayashi, A. F. Molisch, H. V. Poor, and Z. Sahinoglu. Localization via ultra-wideband radios: A look at positioning aspects for future sensor networks. IEEE Signal Processing Magazine, 22 (4):70-84, July 2005.

[13] A. Goldsmith. Wireless Communications. Cambridge University Press, May 2005.

[14] F. Gunnarsson, F. Lindsten, and N. Carlsson. Particle filtering for networkbased positioning terrestrial radio networks. In Proc. IET Conf. Data Fusion Target Tracking 2014: Algorithms and Applications (DF TT 2014), pages 1-7, Liverpool, UK, Apr. 2014.

[15] F. Gustafsson. Particle filter theory and practice with positioning applications. IEEE Aerospace and Electronic Systems Magazine, 25(7):53-82, July 2010 .

[16] F. Gustafsson. Statistical Sensor Fusion. Studentlitteratur, 2010.

[17] F. Gustafsson and F. Gunnarsson. Mobile positioning using wireless networks: Possibilities and fundamental limitations based on available wireless network measurements. IEEE Signal Processing Magazine, 22(4):41-53, July 2005.

[18] R. Jonker and A. Volgenant. A shortest augmenting path algorithm for dense and sparse linear assignment problems. Computing, 38(4):325-340, Dec. 1987.

[19] J. Karedal, S. Wyne, P. Almers, F. Tufvesson, and A. F. Molisch. A measurement-based statistical model for industrial ultra-wideband channels. IEEE transactions on wireless communications, 6(8), 2007.

[20] S. M. Kay. Fundamentals of Statistical Processing, Volume I. Prentice Hall, Mar. 1993.

[21] J. Khodjaev, Y. Park, and A. S. Malik. Survey of NLOS identification and error mitigation problems in UWB-based positioning algorithms for dense environments. Annals of telecommunications - Annales des télécommunications, 65(5-6):301-311, Aug. 2009. 
[22] J. Kulmer, S. Hinteregger, B. Grosswindhager, M. Rath, M. S. Bakr, E. Leitinger, and K. Witrisal. Using DecaWave UWB transceivers for highaccuracy multipath-assisted indoor positioning. In 2017 IEEE International Conference on Communications Workshops (ICC Workshops), pages 12391245, Paris, France, May 2017.

[23] J.-Y. Lee and R. A. Scholtz. Ranging in a dense multipath environment using an UWB radio link. IEEE Journal on Selected Areas in Communications, 20 (9):1677-1683, Dec. 2002.

[24] E. Leitinger, P. Meissner, M. Frohle, and K. Witrisal. Performance bounds for multipath-assisted indoor localization on backscatter channels. In 2014 IEEE Radar Conference, pages 0070-0075, Cincinnati, OH, USA, May 2014.

[25] E. Leitinger, P. Meissner, M. Lafer, and K. Witrisal. Simultaneous localization and mapping using multipath channel information. In 2015 IEEE International Conference on Communication Workshop (ICCW), London, UK, June 2015.

[26] L. Ljung. System Identification: Theory for the User. Prentice Hall, Upper Saddle River, NJ, second edition, Jan. 1999.

[27] E. Ljungzell. Multipath-assisted Single-anchor Outdoor Positioning in Urban Environments. Master's thesis, Linköping University, Automatic Control, 2018.

[28] A. Mannesson. Joint Positioning and Multipath Radio Channel Estimation and Prediction. PhD thesis, Department of Automatic Control, Lund University, Lund, 2016.

[29] P. Meissner. Multipath-Assisted Indoor Positioning. PhD thesis, Graz University of Technology, 2014.

[30] P. Meissner, C. Steiner, and K. Witrisal. UWB positioning with virtual anchors and floor plan information. In 2010 IEEE 7th Workshop on Positioning, Navigation and Communication, pages 150-156, Dresden, Germany, Mar. 2010.

[31] P. Meissner, E. Leitinger, and K. Witrisal. UWB for Robust Indoor Tracking: Weighting of Multipath Components for Efficient Estimation. IEEE Wireless Communications Letters, 3(5):501-504, Oct. 2014.

[32] A. F. Molisch. Wireless Communications. John Wiley \& Sons, Piscataway, NJ, second edition, 2012.

[33] F. Montorsi, F. Pancaldi, and G. M. Vitetta. Statistical Characterization and Mitigation of NLOS Bias in UWB Localization Systems. arXiv:1203.2890 [cs, math, stat], Mar. 2012.

[34] K. G. Murty. An Algorithm for Ranking all the Assignments in Order of Increasing Cost. Operations Research, 16(3):682-687, 1968. 
[35] I. Olofsson. Enhancements in LTE OTDOA Positioning for Multipath Environments. Master's thesis, Linköping University, Communication Systems, 2016.

[36] A. Osseiran, J. F. Monserrat, and P. Marsch. 5G Mobile and Wireless Communications Technology. Cambridge University Press, May 2016.

[37] K. Pahlavan, X. Li, and J. P. Makela. Indoor geolocation science and technology. IEEE Communications Magazine, 40(2):112-118, Feb. 2002.

[38] N. Patwari, J. N. Ash, S. Kyperountas, A. O. Hero, R. L. Moses, and N. S. Correal. Locating the nodes: Cooperative localization in wireless sensor networks. IEEE Signal Processing Magazine, 22(4):54-69, July 2005.

[39] T. Pedersen. Contributions in Radio Channel Sounding, Modeling, and Estimation. PhD thesis, Aalborg University, 2009.

[40] C. R. J. Roger A. Horn. Matrix Analysis. Cambridge University Press, Oct. 2012.

[41] H. Ryden, A. A. Zaidi, S. M. Razavi, F. Gunnarsson, and I. Siomina. Enhanced time of arrival estimation and quantization for positioning in LTE networks. In Proc. and Mobile Radio Communications (PIMRC) 2016 IEEE 27th Annual Int. Symp. Personal, Indoor, Sept. 2016.

[42] S. Särkkä. Bayesian Filtering and Smoothing. Cambride University Press, Sept. 2013.

[43] I. Sharp and K. Yu. Indoor TOA Error Measurement, Modeling, and Analysis. IEEE Transactions on Instrumentation and Measurement, 63(9):2129-2144, Sept. 2014.

[44] Y. Shen and M. Z. Win. Fundamental Limits of Wideband LocalizationPart I: A General Framework. IEEE Transactions on Information Theory, 56 (10):4956-4980, Oct. 2010.

[45] H. L. V. Trees and K. L. Bell. Detection Estimation and Modulation Theory 1. John Wiley \& Sons, May 2013.

[46] D. Tse and P. Viswanath. Fundamentals of Wireless Communication. Cambridge University Press, 2005.

[47] D. Verenzuela, A. Bergström, and E. Björnson. Optimal Power Control for Superimposed Pilots in Uplink Massive MIMO Systems. In 2018 IEEE International Conference Record of the 52nd Asilomar Conference on Signals Systems and Computers, pages 499-503, Pacific Grove, CA, USA, Oct. 2018.

[48] K. Witrisal and P. Meissner. Performance bounds for multipath-assisted indoor navigation and tracking (MINT). In 2012 IEEE International Conference on Communications (ICC), pages 4321-4325, Ottawa, ON, Canada, June 2012. 
[49] K. Witrisal, C. Anton, J. Peral-Rosado, G. Seco-Granados, R. Raulefs, E. Leitinger, S. Grebien, T. Wilding, D. Dardari, E. S. Lohan, H. Wymeersch, J.-J. Floch, A. Tonello, S. Shaikh, T. Zemen, T. Pedersen, T. Fath, C. Fernández-Prades, J. Vilà-Valls, and B. Sackenreuter. Whitepaper on new localization methods for $5 \mathrm{G}$ wireless systems and the internet-of-things. Technical report, COST Action CA15104 (IRACON), Apr. 2018.

[50] L. Yi, S. G. Razul, Z. Lin, and C. M. See. Target Tracking in Mixed LOS/NLOS Environments Based on Individual Measurement Estimation and LOS Detection. IEEE Transactions on Wireless Communications, 13(1):99-111, Jan. 2014. 



\section{Licentiate Theses \\ Division of Automatic Control \\ Linköping University}

P. Andersson: Adaptive Forgetting through Multiple Models and Adaptive Control of Car Dynamics. Thesis No. 15, 1983.

B. Wahlberg: On Model Simplification in System Identification. Thesis No. 47, 1985.

A. Isaksson: Identification of Time Varying Systems and Applications of System Identification to Signal Processing. Thesis No. 75, 1986.

G. Malmberg: A Study of Adaptive Control Missiles. Thesis No. 76, 1986.

S. Gunnarsson: On the Mean Square Error of Transfer Function Estimates with Applications to Control. Thesis No. 90, 1986.

M. Viberg: On the Adaptive Array Problem. Thesis No. 117, 1987.

K. Ståhl: On the Frequency Domain Analysis of Nonlinear Systems. Thesis No. 137, 1988.

A. Skeppstedt: Construction of Composite Models from Large Data-Sets. Thesis No. 149, 1988.

P. A. J. Nagy: MaMiS: A Programming Environment for Numeric/Symbolic Data Processing. Thesis No. 153, 1988.

K. Forsman: Applications of Constructive Algebra to Control Problems. Thesis No. 231, 1990.

I. Klein: Planning for a Class of Sequential Control Problems. Thesis No. 234, 1990.

F. Gustafsson: Optimal Segmentation of Linear Regression Parameters. Thesis No. 246, 1990.

H. Hjalmarsson: On Estimation of Model Quality in System Identification. Thesis No. 251, 1990.

S. Andersson: Sensor Array Processing; Application to Mobile Communication Systems and Dimension Reduction. Thesis No. 255, 1990.

K. Wang Chen: Observability and Invertibility of Nonlinear Systems: A Differential Algebraic Approach. Thesis No. 282, 1991.

J. Sjöberg: Regularization Issues in Neural Network Models of Dynamical Systems. Thesis No. 366, 1993.

P. Pucar: Segmentation of Laser Range Radar Images Using Hidden Markov Field Models. Thesis No. 403, 1993.

H. Fortell: Volterra and Algebraic Approaches to the Zero Dynamics. Thesis No. 438, 1994.

T. McKelvey: On State-Space Models in System Identification. Thesis No. 447, 1994.

T. Andersson: Concepts and Algorithms for Non-Linear System Identifiability. Thesis No. 448, 1994.

P. Lindskog: Algorithms and Tools for System Identification Using Prior Knowledge. Thesis No. 456, 1994.

J. Plantin: Algebraic Methods for Verification and Control of Discrete Event Dynamic Systems. Thesis No. 501, 1995.

J. Gunnarsson: On Modeling of Discrete Event Dynamic Systems, Using Symbolic Algebraic Methods. Thesis No. 502, 1995.

A. Ericsson: Fast Power Control to Counteract Rayleigh Fading in Cellular Radio Systems. Thesis No. 527, 1995.

M. Jirstrand: Algebraic Methods for Modeling and Design in Control. Thesis No. 540, 1996.

K. Edström: Simulation of Mode Switching Systems Using Switched Bond Graphs. Thesis No. 586, 1996. 
J. Palmqvist: On Integrity Monitoring of Integrated Navigation Systems. Thesis No. 600, 1997.

A. Stenman: Just-in-Time Models with Applications to Dynamical Systems. Thesis No. 601, 1997.

M. Andersson: Experimental Design and Updating of Finite Element Models. Thesis No. 611, 1997.

U. Forssell: Properties and Usage of Closed-Loop Identification Methods. Thesis No. 641, 1997.

M. Larsson: On Modeling and Diagnosis of Discrete Event Dynamic systems. Thesis No. 648, 1997.

N. Bergman: Bayesian Inference in Terrain Navigation. Thesis No. 649, 1997.

V. Einarsson: On Verification of Switched Systems Using Abstractions. Thesis No. 705, 1998.

J. Blom, F. Gunnarsson: Power Control in Cellular Radio Systems. Thesis No. 706, 1998.

P. Spångéus: Hybrid Control using LP and LMI methods - Some Applications. Thesis No. 724, 1998.

M. Norrlöf: On Analysis and Implementation of Iterative Learning Control. Thesis No. 727, 1998.

A. Hagenblad: Aspects of the Identification of Wiener Models. Thesis No. 793, 1999.

F. Tjärnström: Quality Estimation of Approximate Models. Thesis No. 810, 2000.

C. Carlsson: Vehicle Size and Orientation Estimation Using Geometric Fitting. Thesis No. 840, 2000.

J. Löfberg: Linear Model Predictive Control: Stability and Robustness. Thesis No. 866, 2001.

O. Härkegård: Flight Control Design Using Backstepping. Thesis No. 875, 2001.

J. Elbornsson: Equalization of Distortion in A/D Converters. Thesis No. 883, 2001.

J. Roll: Robust Verification and Identification of Piecewise Affine Systems. Thesis No. 899, 2001.

I. Lind: Regressor Selection in System Identification using ANOVA. Thesis No. 921, 2001.

R. Karlsson: Simulation Based Methods for Target Tracking. Thesis No. 930, 2002.

P.-J. Nordlund: Sequential Monte Carlo Filters and Integrated Navigation. Thesis No. 945, 2002.

M. Östring: Identification, Diagnosis, and Control of a Flexible Robot Arm. Thesis No. 948, 2002.

C. Olsson: Active Engine Vibration Isolation using Feedback Control. Thesis No. 968, 2002.

J. Jansson: Tracking and Decision Making for Automotive Collision Avoidance. Thesis No. 965, 2002.

N. Persson: Event Based Sampling with Application to Spectral Estimation. Thesis No. 981, 2002.

D. Lindgren: Subspace Selection Techniques for Classification Problems. Thesis No. 995, 2002.

E. Geijer Lundin: Uplink Load in CDMA Cellular Systems. Thesis No. 1045, 2003.

M. Enqvist: Some Results on Linear Models of Nonlinear Systems. Thesis No. 1046, 2003.

T. Schön: On Computational Methods for Nonlinear Estimation. Thesis No. 1047, 2003.

F. Gunnarsson: On Modeling and Control of Network Queue Dynamics. Thesis No. 1048, 2003.

S. Björklund: A Survey and Comparison of Time-Delay Estimation Methods in Linear Systems. Thesis No. 1061, 2003. 
M. Gerdin: Parameter Estimation in Linear Descriptor Systems. Thesis No. 1085, 2004.

A. Eidehall: An Automotive Lane Guidance System. Thesis No. 1122, 2004.

E. Wernholt: On Multivariable and Nonlinear Identification of Industrial Robots. Thesis No. 1131, 2004.

J. Gillberg: Methods for Frequency Domain Estimation of Continuous-Time Models. Thesis No. 1133, 2004.

G. Hendeby: Fundamental Estimation and Detection Limits in Linear Non-Gaussian Systems. Thesis No. 1199, 2005.

D. Axehill: Applications of Integer Quadratic Programming in Control and Communication. Thesis No. 1218, 2005.

J. Sjöberg: Some Results On Optimal Control for Nonlinear Descriptor Systems. Thesis No. 1227, 2006.

D. Törnqvist: Statistical Fault Detection with Applications to IMU Disturbances. Thesis No. 1258, 2006.

H. Tidefelt: Structural algorithms and perturbations in differential-algebraic equations. Thesis No. 1318, 2007.

S. Moberg: On Modeling and Control of Flexible Manipulators. Thesis No. 1336, 2007.

J. Wallén: On Kinematic Modelling and Iterative Learning Control of Industrial Robots. Thesis No. 1343, 2008.

J. Harju Johansson: A Structure Utilizing Inexact Primal-Dual Interior-Point Method for Analysis of Linear Differential Inclusions. Thesis No. 1367, 2008.

J. D. Hol: Pose Estimation and Calibration Algorithms for Vision and Inertial Sensors. Thesis No. 1370, 2008.

H. Ohlsson: Regression on Manifolds with Implications for System Identification. Thesis No. 1382, 2008.

D. Ankelhed: On low order controller synthesis using rational constraints. Thesis No. 1398, 2009.

P. Skoglar: Planning Methods for Aerial Exploration and Ground Target Tracking. Thesis No. 1420, 2009.

C. Lundquist: Automotive Sensor Fusion for Situation Awareness. Thesis No. 1422, 2009.

C. Lyzell: Initialization Methods for System Identification. Thesis No. 1426, 2009.

R. Falkeborn: Structure exploitation in semidefinite programming for control. Thesis No. 1430, 2010.

D. Petersson: Nonlinear Optimization Approaches to $\mathcal{H}_{2}$-Norm Based LPV Modelling and Control. Thesis No. 1453, 2010.

Z. Sjanic: Navigation and SAR Auto-focusing in a Sensor Fusion Framework. Thesis No. 1464, 2011.

K. Granström: Loop detection and extended target tracking using laser data. Thesis No. 1465, 2011.

J. Callmer: Topics in Localization and Mapping. Thesis No. 1489, 2011.

F. Lindsten: Rao-Blackwellised particle methods for inference and identification. Thesis No. 1480, 2011.

M. Skoglund: Visual Inertial Navigation and Calibration. Thesis No. 1500, 2011.

S. Khoshfetrat Pakazad: Topics in Robustness Analysis. Thesis No. 1512, 2011.

P. Axelsson: On Sensor Fusion Applied to Industrial Manipulators. Thesis No. 1511, 2011.

A. Carvalho Bittencourt: On Modeling and Diagnosis of Friction and Wear in Industrial Robots. Thesis No. 1516, 2012.

P. Rosander: Averaging level control in the presence of frequent inlet flow upsets. Thesis No. 1527, 2012. 
N. Wahlström: Localization using Magnetometers and Light Sensors. Thesis No. 1581, 2013.

R. Larsson: System Identification of Flight Mechanical Characteristics. Thesis No. 1599, 2013.

Y. Jung: Estimation of Inverse Models Applied to Power Amplifier Predistortion. Thesis No. 1605, 2013.

M. Syldatk: On Calibration of Ground Sensor Networks. Thesis No. 1611, 2013.

M. Roth: Kalman Filters for Nonlinear Systems and Heavy-Tailed Noise. Thesis No. 1613, 2013.

D. Simon: Model Predictive Control in Flight Control Design - Stability and Reference Tracking. Thesis No. 1642, 2014.

J. Dahlin: Sequential Monte Carlo for inference in nonlinear state space models. Thesis No. 1652, 2014.

M. Kok: Probabilistic modeling for positioning applications using inertial sensors. Thesis No. 1656, 2014.

J. Linder: Graybox Modelling of Ships Using Indirect Input Measurements. Thesis No. 1681, 2014.

G. Mathai: Direction of Arrival Estimation of Wideband Acoustic Wavefields in a Passive Sensing Environment. Thesis No. 1721, 2015.

I. Nielsen: On Structure Exploiting Numerical Algorithms for Model Predictive Control. Thesis No. 1727, 2015.

C. Veibäck: Tracking of Animals Using Airborne Cameras. Thesis No. 1761, 2016.

N. Evestedt: Sampling Based Motion Planning for Heavy Duty Autonomous Vehicles. Thesis No. 1762, 2016.

H. Nyqvist: On Pose Estimation in Room-Scaled Environments. Thesis No. 1765, 2016.

Y. Zhao: Position Estimation in Uncertain Radio Environments and Trajectory Learning. Thesis No. 1772, 2017.

P. Kasebzadeh: Parameter Estimation for Mobile Positioning Applications. Thesis No. 1786, 2017.

K. Radnosrati: On Timing-Based Localization in Cellular Radio Networks. Thesis No. 1808, 2018.

G. Lindmark: Methods and Algorithms for Control Input Placement in Complex Networks. Thesis No. 1814, 2018.

M. Lindfors: Frequency Tracking for Speed Estimation. Thesis No. 1815, 2018.

D. Ho: Some results on closed-loop identification of quadcopters. Thesis No. 1826, 2018.

O. Ljungqvist: On motion planning and control for truck and trailer systems. Thesis No. 1832, 2019.

P. Boström-Rost: On Informative Path Planning for Tracking and Surveillance. Thesis No. 1838, 2019.

K. Bergman: On Motion Planning Using Numerical Optimal Control. Thesis No. 1843, 2019.

M. Klingspor: Low-rank optimization in system identification. Thesis No. 1855, 2019. 


\section{FACULTY OF SCIENCE AND ENGINEERING}

Linköping studies in science and technology. Licentiate Thesis No. 1867

Department of Electrical Engineering

Linköping University

SE-581 83 Linköping, Sweden

www.liu.se 